Portland State University

PDXScholar

Dissertations and Theses

Dissertations and Theses

Spring 5-24-2017

\title{
Communicating at Terahertz Frequencies
}

Farnoosh Moshirfatemi

Portland State University

Follow this and additional works at: https://pdxscholar.library.pdx.edu/open_access_etds

Part of the Computer Sciences Commons

Let us know how access to this document benefits you.

Recommended Citation

Moshirfatemi, Farnoosh, "Communicating at Terahertz Frequencies" (2017). Dissertations and Theses.

Paper 3641.

https://doi.org/10.15760/etd.5525

This Dissertation is brought to you for free and open access. It has been accepted for inclusion in Dissertations and Theses by an authorized administrator of PDXScholar. Please contact us if we can make this document more accessible: pdxscholar@pdx.edu. 
Communicating at Terahertz Frequencies

$$
\text { by }
$$

Farnoosh Moshirfatemi

A dissertation submitted in partial fulfillment of the requirements for the degree of

\author{
Doctor of Philosophy \\ in
}

Computer Science

\author{
Dissertation Committee: \\ Suresh Singh, Chair \\ Fei Xie \\ Feng Liu \\ Lisa Zurk
}

Portland State University

2017 


\begin{abstract}
The number of users who get access to wireless links is increasing each day and many new applications require very high data rates. The increasing demand for higher data rates has led to the development of new techniques to increase spectrum efficiency to achieve this goal. However, the limited bandwidth of the frequency bands that are currently used for wireless communication bounds the maximum data rate possible.

In the past few years, researchers have developed new devices that work as Terahertz $(\mathrm{THz})$ transmitters and receivers. The development of these devices and the large available bandwidth of the $\mathrm{THz}$ band is a possible solution to this ever increasing demand. However, $\mathrm{THz}$ communication is still in its infancy and more research needs to be done to bring $\mathrm{THz}$ technology into every day life.

In this research, we study wireless $\mathrm{THz}$ communication systems. As the first step, we conducted detailed channel measurements to study and analyze the characteristics of $\mathrm{THz}$ signals under different channel conditions. These propagation models mimic the behavior of $\mathrm{THz}$ signals in real applications.

Then we use these models to study appropriate modulation methods for directional and omni-directional $\mathrm{THz}$ channels. We also use pulsed $\mathrm{THz}$ signals in wireless communication channels to send data at a very high rate. We have developed rate adaptation algorithms to allow multiple users to share the same $\mathrm{THz}$ channel for downlink applications while fairness is maintained among them.
\end{abstract}




\section{DEDICATION}

I dedicate this thesis to my parents and my husband for all their support and love. 


\section{ACKNOWLEDGMENTS}

I would like to express my most profound gratitudes to my advisor, Professor Suresh Singh, for his patience and guidance during my Ph.D. program at the Portland State University (PSU). Working with Professor Singh has been one of the most valuable experiences. I am particularly grateful for the generous amount of time he dedicated towards my research work, and for the enthusiasm and interest he showed in my work. His comments and ideas have shaped this thesis in many ways. It has been an honor and privilege for me to be Professor Singh's Ph.D. student.

My deepest gratitude goes to Dr. Fei Xie, Dr. Feng Liu, and Dr. Lisa Zurk for having been on my dissertation committee. Also I need to thank Dr. Lisa Zurk for letting us use her Terahertz lab for our measurements.

I would like to thank my family for their continuous support, encouragement and love. My Special thanks to my lovely husband, Dr. Hooman Shirani-Mehr for always being there and supporting me. He walked beside me in this long journey. My deepest thanks to my parents for all the sacrifices they have made that enabled me to earn my Ph.D. degree. 


\section{TABLE OF CONTENTS}

Abstract $\quad$ i

Dedication $\quad$ ii

Acknowledgments $\quad$ iii

List of Tables vii

List of Figures $\quad$ viii

Chapter 1 Introduction 1

1.1 Why Do We Need Higher Data Rates . . . . . . . . . . . . . . . . . 1

1.2 What Is Terahertz . . . . . . . . . . . . . . . . 3

1.3 Practical Problems Of Using Terahertz . . . . . . . . . . . . . 4

1.4 Proposed Research . . . . . . . . . . . . . . . . . 7

1.5 Major Research Contributions . . . . . . . . . . . . . . . . 8

1.6 Thesis Organization . . . . . . . . . . . . . . . 8

$\begin{array}{lll}\text { Chapter } 2 & \text { Overview of Communication Systems } & 10\end{array}$

2.1 Fourier Transform . . . . . . . . . . . . . . . . . . . 13

2.2 Energy and Power of the Signal . . . . . . . . . . . . . . . . . . 14

2.3 The Channel . . . . . . . . . . . . . . . . . . . . . 15

2.3.1 Channel Modeling . . . . . . . . . . . . . . 17

2.4 Pulse Shaping (Filter) . . . . . . . . . . . . . . . . . . . 19

2.4.1 Raised Cosine Filter . . . . . . . . . . . . . . 20

2.4 .2 Matched Filter . . . . . . . . . . . . . . . 20

2.5 Continuous Wave Modulation Methods . . . . . . . . . . . . . . . . 21

2.5.1 Phase Shift Keying (PSK) . . . . . . . . . . . . . . . 23

2.5.2 Quadrature Amplitude Modulation (QAM) . . . . . . . . 25

2.6 Pulsed Signal Modulation Methods . . . . . . . . . . . . . . . . 27 
2.6.1 Amplitude Shift Keying (ASK) _ . . . . . . . . . 28

2.6 .2 On-Off Keying (OOK) . . . . . . . . . . . . . . . . . 29

2.6.3 Frequency-Domain Differential PSK (FD-DPSK) . . . . . 30

Chapter 3 Channel Characterization 34

3.1 Testbed and Experimental Design . . . . . . . . . . . . . 34

3.2 Overview of the Received Signals _ . . . . . . . . . . . 37

3.3 Directional Channel Characterization . . . . . . . . . . . 40

3.3.1 Modeling Attenuation Due to Absorption . . . . . . . . . 47

3.3.2 Modeling of Collimating Lenses Divergence . . . . . . . . . 55

3.3.3 Summary of the Directional Channel Model . . . . . . . . 58

3.4 Non-directional Channel Characterization . . . . . . . . . . . 61

3.4.1 Antenna Characterization . . . . . . . . . . . . 66

3.4.2 Summary of the Non-Directional Channel Model . . . . . . . 75

3.5 Summary . . . . . . . . . . . . . . . . . 75

Chapter 4 Directional Communication Models 76

4.1 FD-DPSK Modulation . . . . . . . . . . . . . 76

4.1.1 FD-DMPSK Modulation . . . . . . . . . . . . . 77

4.1.2 Simulation Results for FD-DMPSK . . . . . . . . . . . 82

4.1.3 Adaptive FD-DPSK Modulation . . . . . . . . . . . . . . 84

4.1.4 Simulation Results for Adaptive FD-MDPSK Modulation . . 87

4.1.5 Multiple Transmitters and Achievable Data Rates Based on

Adaptive FD-DPSK Modulation . . . . . . . . . . . . . 89

4.1.6 Achievable Data Rates in Directional THz Channels . . . . . 90

4.1.7 Data Rate for Cities Based on Adaptive FD-DQPSK . . . . 92

4.1.8 Multiple Users in FD-DPSK Modulation . . . . . . . . . . 93

4.2 ASK Modulation Methods . . . . . . . . . . . . . . 95

4.2 .1 3ASK Modulation . . . . . . . . . . . . . . . . 95

4.2 .2 5ASK Modulation . . . . . . . . . . . . . . . . . 98

4.2 .3 ASK Modulation Enhancement . . . . . . . . . . . . . . 102

4.2.4 Data Rate for Cities Based on Enhanced ASK Modulation . 111

4.3 Summary . . . . . . . . . . . . . . . . . . 111

Chapter 5 Omni-Directional Communication Models 114

5.1 Omni-directional Channel Characterization and Modeling . . . . . . 114 
5.2 Rate Adaptation Algorithms . . . . . . . . . . . . . . . 117

5.2.1 Single Channel Algorithm (SCA) . . . . . . . . . . . 118

5.2 .2 Multiple Channel Algorithm (MCA) . . . . . . . . . . 119

5.3 Simulation Results . . . . . . . . . . . . . . . . . . . . 121

5.3 .1 SCA Simulation Results . . . . . . . . . . . . . 124

5.3 .2 MCA Simulation Results . . . . . . . . . . . . . . . 127

5.4 The Effect of Transmitted Power on transmitter-receiver distance . 128

5.5 Summary . . . . . . . . . . . . . . . . . . . . . 129

Chapter 6 Literature Review 131

6.1 Literature Review On Terahertz Generation and Detection Techniques 131

6.1.1 THz Generation Techniques . . . . . . . . . . . . . . . 131

$6.1 .2 \mathrm{THz}$ Detection Techniques . . . . . . . . . . . . . . . 136

6.2 Literature Review On Achieved Data Rates . . . . . . . . . . . 141

$\begin{array}{lll}\text { Chapter } 7 & \text { Conclusion } & 145\end{array}$

$\begin{array}{lll}\text { Appendix A Time Domain Signals } & 158\end{array}$

$\begin{array}{lll}\text { Appendix B Atmospheric Attenuation Coefficient } & 159\end{array}$

$\begin{array}{lll}\text { Appendix C } & \text { Raised Cosine Filter } & 166\end{array}$

$\begin{array}{lll}\text { Appendix D } & \text { FD-DPSK Modulation } & 168\end{array}$

Appendix E NS3:THz Propagation Loss Model C++ Header File 179

Appendix F NS3: THz Propagation Loss Model C++ Source Code

Appendix G NS3:THz Propagation Loss Model Test 220

Appendix H NS3:THz vs. Friis Propagation Loss Model Example 231 


\section{LIST OF TABLES}

Table 3.1 Summary of variable parameters. . . . . . . . . . . . . . 36

Table 3.2 HITRAN line by line parameters . . . . . . . . . . . . . 48

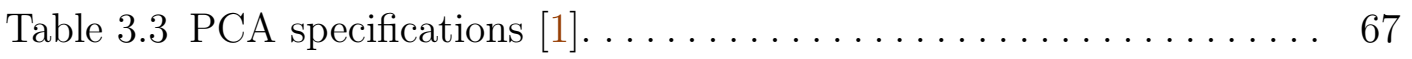

Table 4.1 The list of figures that show BER vs $\mathrm{E}_{\mathrm{b}} / \mathrm{N}_{0}$ for different modulation schemes corresponding to transmitter-receiver ( $\mathrm{Tx}-\mathrm{Rx}$ ) distances of

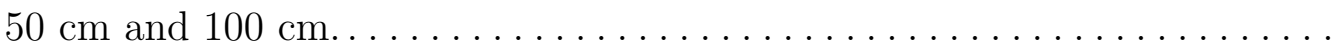

Table 4.2 Number of erroneous bands with $\mathrm{E}_{\mathrm{b}} / \mathrm{N}_{0}=0$ and BER $\geq 10^{-2}$ for FD-DPSK scheme and transmitter-receiver ( $\mathrm{Tx}-\mathrm{Rx})$ distances of $50 \mathrm{~cm}$

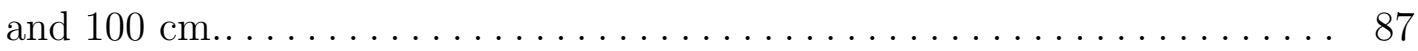

Table 4.3 Adaptive FD-DQPSK bit rate for multiple scenarios with $\mathrm{E}_{\mathrm{b}} / \mathrm{N}_{0}=$

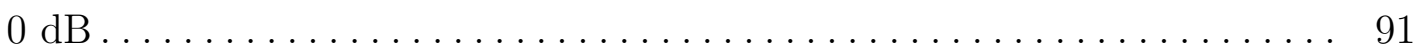

Table 4.4 Relative Humidity $(\mathrm{RH})$ for cities in summer and winter . . . . 92

Table 6.1 A summary of related works. CMOS: complementary metal oxide semiconductor, MMIC: monolithic microwave integrated circuits, SBD: schottky barrier diode........................... 144 


\section{LIST OF FIGURES}

Figure 1.1 Terahertz spectrum sits between microwave and infrared re-

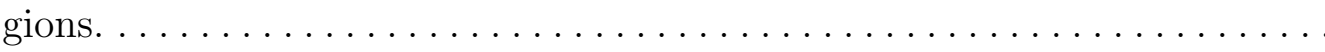

Figure 1.2 The effect of a smooth (on the left) and a rough (on the right) surface on the reflected signal $[48] \ldots \ldots \ldots \ldots \ldots \ldots \ldots \ldots \ldots \ldots \ldots \ldots \ldots \ldots \ldots$

Figure 1.3 Atmospheric attenuation as a function of frequency and distance in the range of $0-1 \mathrm{THz}[33] \ldots \ldots \ldots \ldots \ldots \ldots \ldots \ldots \ldots \ldots \ldots \ldots \ldots \ldots \ldots$

Figure 1.4 Atmospheric transmission of THz signal within 0-5 THz [71]. . 7

Figure 2.1 Block diagram of a communication system............ 11

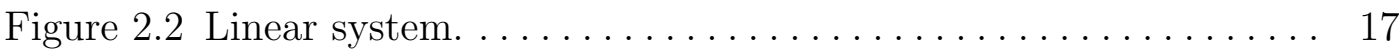

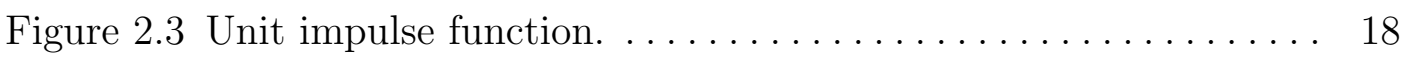

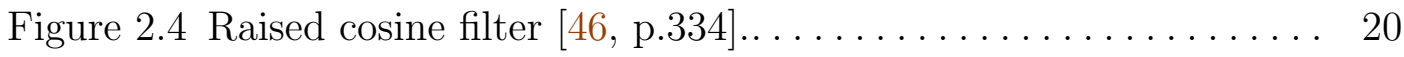

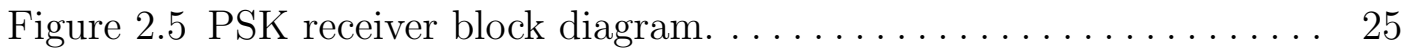

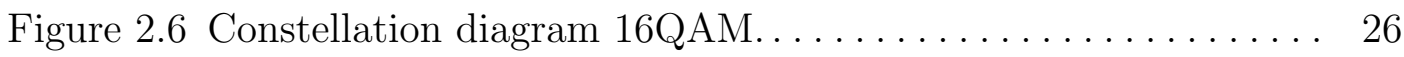

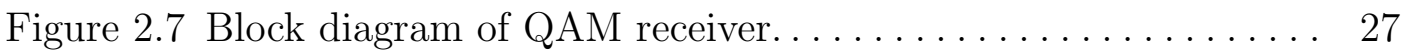

Figure 2.8 Block diagram of a correlator receiver in ASK modulation. . . 28

Figure 2.9 An example of OOK modulation [9]. . . . . . . . . . . . . 29

Figure 2.10 The block diagram of OOK receiver. . . . . . . . . . . . 30

Figure 2.11 Transmitter structure using an AOM. . . . . . . . . . . 31

Figure 3.1 The lab setup for creating dry channel. . . . . . . . . . . . 36

Figure 3.2 Received pulse in directional channel for transmitter-receiver

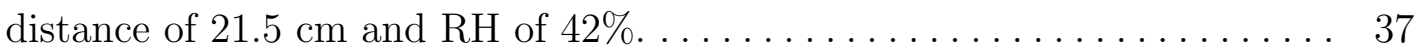

Figure 3.3 Received pulse in directional channel for transmitter-receiver

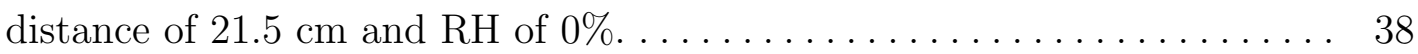

Figure 3.4 Received pulse in non-directional channel for transmitter-

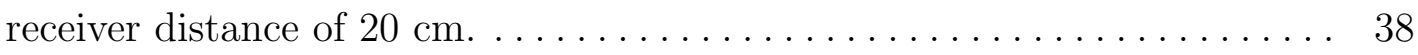

Figure 3.5 Filtered received pulse for directional channel when transmitterreceiver distance is $21.5 \mathrm{~cm}$ and $\mathrm{RH}$ is equal to $42 \% \ldots \ldots \ldots \ldots \ldots$ 
Figure 3.6 Filtered received pulse for directional channel when transmitterreceiver distance is $21.5 \mathrm{~cm}$ and $\mathrm{RH}$ is equal to $0 \% \ldots \ldots \ldots \ldots \ldots \ldots$ Figure 3.7 Filtered received pulse for non-directional channel when transmitter-

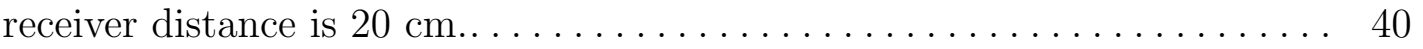

Figure 3.8 Power vs. transmitter-receiver distance of the received signal in directional channel for $0 \%$ and $42 \%$ relative humidities. . . . . . . . . 41

Figure 3.9 Comparison of the frequency spectrum of the signals with $42 \%$ and $0 \%$ relative humidity $(\mathrm{RH})$ with transmitter-receiver distance of 21.5 $\mathrm{cm}$. The solid line corresponds to signal with $42 \% \mathrm{RH}$ and the dotted line corresponds to the signal with $0 \% \mathrm{RH} \ldots \ldots \ldots \ldots \ldots \ldots \ldots \ldots \ldots \ldots \ldots \ldots$

Figure 3.10 Received power of $110 \mathrm{GHz}$ band with $1 \mathrm{GHz}$ bandwidth for

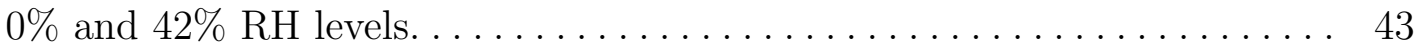

Figure 3.11 Received power of $170 \mathrm{GHz}$ band with $1 \mathrm{GHz}$ bandwidth for $0 \%$ and $42 \%$ RH levels. . . . . . . . . . . . . . . . . . 43

Figure 3.12 Received power of $400 \mathrm{GHz}$ band with $1 \mathrm{GHz}$ bandwidth for

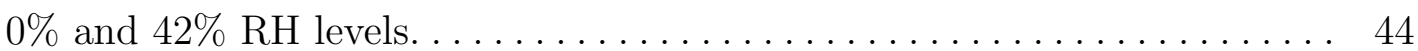

Figure 3.13 Received power of $560 \mathrm{GHz}$ band with $1 \mathrm{GHz}$ bandwidth for $0 \%$ and $42 \%$ RH levels. . . . . . . . . . . . . . . . .

Figure 3.14 Received power of $750 \mathrm{GHz}$ band with $1 \mathrm{GHz}$ bandwidth for

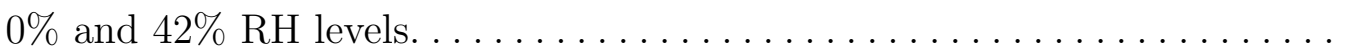

Figure 3.15 Received power of $1.1 \mathrm{THz}(1100 \mathrm{GHz})$ band with $1 \mathrm{GHz}$

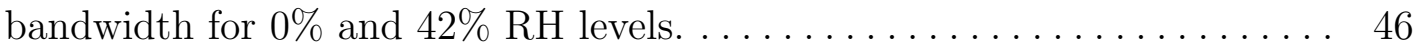
Figure 3.16 Water vapor absorption coefficient for $42 \%$ relative humidity

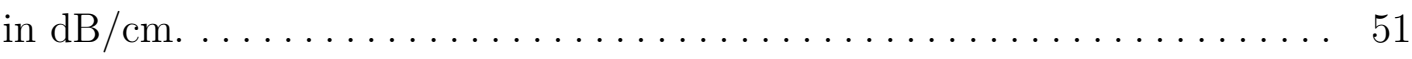

Figure 3.17 Oxygen absorption coefficient in $\mathrm{dB} / \mathrm{cm} \ldots \ldots \ldots \ldots \ldots \ldots 52$

Figure 3.18 Nitrogen absorption coefficient in $\mathrm{dB} / \mathrm{cm} \ldots \ldots \ldots \ldots \ldots \ldots$

Figure 3.19 Carbon dioxide absorption coefficient in $\mathrm{dB} / \mathrm{cm} . \ldots \ldots \ldots . . \ldots 3$

Figure 3.20 Atmospheric attenuation coefficient for different relative humidity $(\mathrm{RH})$ levels........................... 54

Figure 3.21 Diverging factor that causes attenuation in the received signal

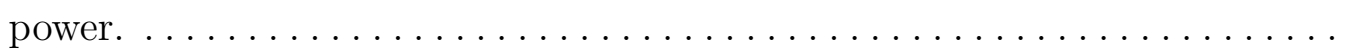
55

Figure 3.22 Confidence interval on the average value of diverging factor found from (3.15) for distances 11.5 and $66.5 \mathrm{~cm}$ when $\mathrm{RH}$ is $0 . \ldots \ldots 57$ Figure 3.23 A comparison of the experimental and the simulation result of the received power in $400 \mathrm{GHz}$ frequency when $\mathrm{RH}$ is $0 \% \ldots \ldots \ldots \ldots$ 
Figure 3.24 Simulated vs Experimental signal over $50 \mathrm{~cm}$ distance and $0 \%$ RH. Solid and dotted line represent simulation and experimental results,

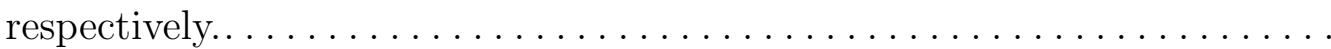

Figure 3.25 Simulated vs Experimental signal over $50 \mathrm{~cm}$ distance and $42 \% \mathrm{RH}$. Solid and dotted line represent simulation and experimental re-

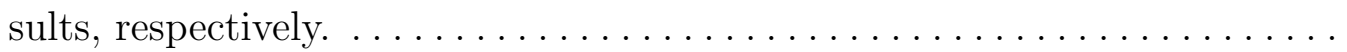

Figure 3.26 Received power vs. transmitter-receiver distance in nondirectional channel when humidity is $42 \% \ldots \ldots \ldots \ldots \ldots \ldots \ldots \ldots$

Figure 3.27 Frequency spectrum of the signal in non-directional channel with transmitter-receiver distance of $50 \mathrm{~cm}$ and $42 \% \mathrm{RH} \ldots \ldots \ldots \ldots \ldots 2$

Figure 3.28 Experimental received power and the fitting function using Matlab"cftool"............................... 63

Figure 3.29 Curve fitting with the second part of the $(3.19) \ldots \ldots \ldots \ldots .64$

Figure 3.30 Experimental received power for $110 \mathrm{GHz}$ band along with

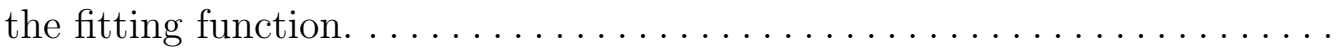

Figure 3.31 Experimental received power for $170 \mathrm{GHz}$ band along with

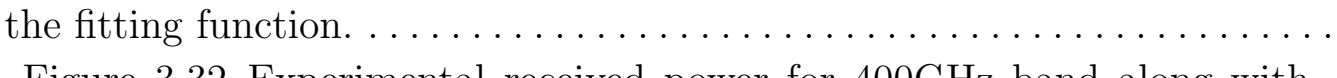

Figure 3.32 Experimental received power for $400 \mathrm{GHz}$ band along with

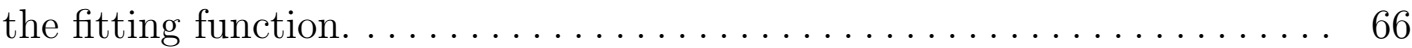

Figure 3.33 Antenna PCA structure $[1] \ldots \ldots \ldots \ldots \ldots \ldots \ldots \ldots \ldots \ldots$

Figure 3.34 Picotemrix transmitter with PCA. . . . . . . . . . . . 68

Figure 3.35 Picotemrix transmitter with PCA and HDPA lens. . . . . . . 68

Figure 3.36 Lab setup for characterizing the antenna. . . . . . . . . . . 68

Figure 3.37 transmitter-receiver angle. . . . . . . . . . . . . . 69

Figure 3.38 Received power in watt when receiver has collimating lens. . 70

Figure 3.39 Received power in watt when receiver has no collimating lens. 70

Figure 3.40 Received power in watt for $300 \mathrm{GHz}$ frequency band when

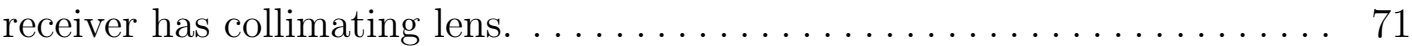

Figure 3.41 Received power in watt for $300 \mathrm{GHz}$ frequency band when

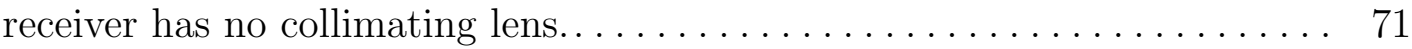

Figure 3.42 Received power in watt when receiver has collimating lens and transmitter and receiver heads are rotated $90^{\circ} \ldots \ldots \ldots \ldots \ldots \ldots . \ldots 2$

Figure 3.43 Received power in watt when receiver has no collimating lens transmitter and receiver heads are rotated $90^{\circ} \ldots \ldots \ldots \ldots \ldots \ldots \ldots \ldots$ 
Figure 3.44 Received power in watt for $300 \mathrm{GHz}$ band when receiver has collimating lens and transmitter and receiver are rotated $90^{\circ} \ldots \ldots \ldots \ldots$ 73 Figure 3.45 Received power in watt for $300 \mathrm{GHz}$ band when receiver has no collimating lens transmitter and receiver are rotated $90^{\circ}$. . . . . . . Figure 3.46 Comparison of the normalized received power for $300 \mathrm{GHz}$ band from (3.30) with the lab measurements when receiver has collimating

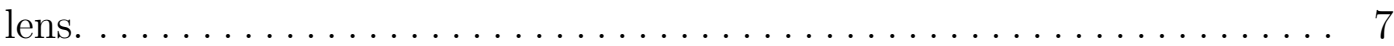

Figure 4.1 Transmitter block diagram. . . . . . . . . . . . . . . 78

Figure 4.2 Receiver block diagram. . . . . . . . . . . . . . . . . . . . 79

Figure 4.3 transmitter, receiver, and channel block diagram for FDD $m$ PSK, where $m$ is $2,4,8,16$, and $64 \ldots \ldots \ldots \ldots \ldots \ldots$

Figure $4.4 \mathrm{BER}$ vs $\mathrm{E}_{\mathrm{b}} / \mathrm{N}_{0}$ for $0 \% \mathrm{RH}$ and transmitter-receiver distance

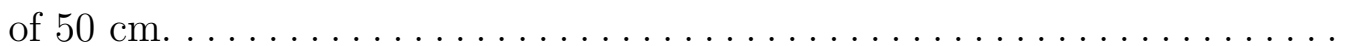

Figure $4.5 \mathrm{BER}$ vs $\mathrm{E}_{\mathrm{b}} / \mathrm{N}_{0}$ for $40 \% \mathrm{RH}$ and transmitter-receiver distance

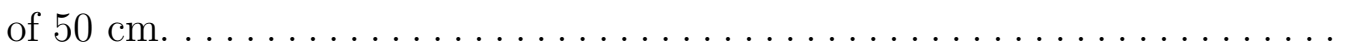

Figure 4.6 BER vs $\mathrm{E}_{\mathrm{b}} / \mathrm{N}_{0}$ for $80 \% \mathrm{RH}$ and transmitter-receiver distance

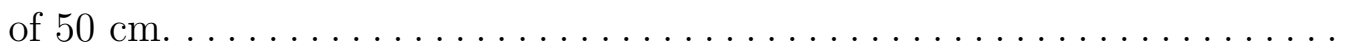

Figure $4.7 \mathrm{BER}$ vs $\mathrm{E}_{\mathrm{b}} / \mathrm{N}_{0}$ for $0 \% \mathrm{RH}$ and transmitter-receiver distance

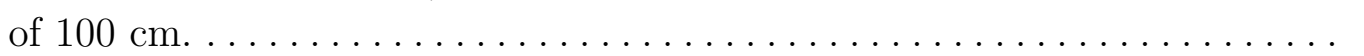

Figure $4.8 \mathrm{BER}$ vs $\mathrm{E}_{\mathrm{b}} / \mathrm{N}_{0}$ for $40 \% \mathrm{RH}$ and transmitter-receiver distance

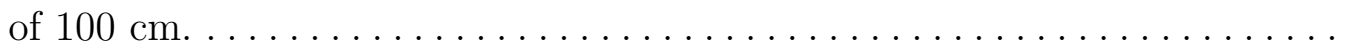

Figure $4.9 \mathrm{BER}$ vs $\mathrm{E}_{\mathrm{b}} / \mathrm{N}_{0}$ for $80 \% \mathrm{RH}$ and transmitter-receiver distance

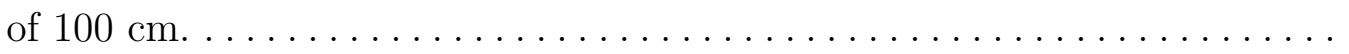
85 Figure 4.10 Bands' error rate vs water vapor absorption coefficient for $\mathrm{E}_{\mathrm{b}} / \mathrm{N}_{0}=0 \mathrm{~dB}$, transmitter-receiver distance $=100 \mathrm{~cm}$, and $\mathrm{RH}=80 \% \ldots \ldots$ Figure 4.11 BER vs $\mathrm{E}_{\mathrm{b}} / \mathrm{N}_{0}$ corresponding adaptive modulation schemes for $40 \% \mathrm{RH}$ and transmitter-receiver distance of $50 \mathrm{~cm} . \ldots \ldots \ldots \ldots$

Figure 4.12 BER vs $\mathrm{E}_{\mathrm{b}} / \mathrm{N}_{0}$ corresponding adaptive modulation schemes for $80 \% \mathrm{RH}$ and transmitter-receiver distance of $50 \mathrm{~cm} \ldots \ldots \ldots \ldots \ldots 8$

Figure 4.13 BER vs $\mathrm{E}_{\mathrm{b}} / \mathrm{N}_{0}$ corresponding adaptive modulation schemes for $40 \% \mathrm{RH}$ and transmitter-receiver distance of $100 \mathrm{~cm}$. . . . . . . . . 89

Figure $4.14 \mathrm{BER}$ vs $\mathrm{E}_{\mathrm{b}} / \mathrm{N}_{0}$ corresponding adaptive modulation schemes for $80 \% \mathrm{RH}$ and transmitter-receiver distance of $100 \mathrm{~cm} . \ldots \ldots \ldots \ldots$. . . Figure 4.15 Up to 10'000 transmitters can generate pulses in a TDM system and achieve higher data rates. . . . . . . . . . . . 
Figure 4.16 Adaptive FD-DQPSK modulation data rate vs distance $(\mathrm{cm})$

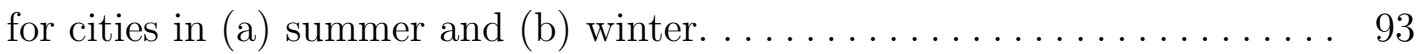
Figure 4.17 Using multiple transmitters.. . . . . . . . . . . . . . 94 Figure 4.18 BER vs $\mathrm{E}_{\mathrm{b}} / \mathrm{N}_{0}$ corresponding to $3 \mathrm{ASK}$ modulation for different RH levels and transmitter-receiver distance of $11.5 \mathrm{~cm} \ldots \ldots \ldots \ldots . .98$ Figure 4.19 BER vs $\mathrm{E}_{\mathrm{b}} / \mathrm{N}_{0}$ corresponding to $3 \mathrm{ASK}$ modulation for different RH levels and transmitter-receiver distance of $66.5 \mathrm{~cm} \ldots \ldots \ldots \ldots \ldots 9$ Figure 4.20 BER vs $\mathrm{E}_{\mathrm{b}} / \mathrm{N}_{0}$ corresponding to $5 \mathrm{ASK}$ modulation for different RH levels and transmitter-receiver distance of $11.5 \mathrm{~cm} . . \ldots \ldots \ldots \ldots 101$ Figure 4.21 BER vs $\mathrm{E}_{\mathrm{b}} / \mathrm{N}_{0}$ corresponding to $5 \mathrm{ASK}$ modulation for different RH levels and transmitter-receiver distance of $66.5 \mathrm{~cm} \ldots \ldots \ldots \ldots \ldots 1$ Figure 4.22 Error percentage of all bands when $\mathrm{RH}=40 \%$, transmitter-

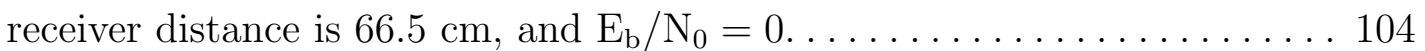
Figure 4.23 Error percentage of erroneous bands when $\mathrm{RH}=40 \%$, transmitterreceiver distance is $66.5 \mathrm{~cm}$, and $\mathrm{E}_{\mathrm{b}} / \mathrm{N}_{0}=0 \ldots \ldots \ldots \ldots \ldots \ldots \ldots \ldots \ldots \ldots \ldots \ldots \ldots \ldots$ Figure 4.24 Enhanced BER based on 3ASK modulation for transmitter-

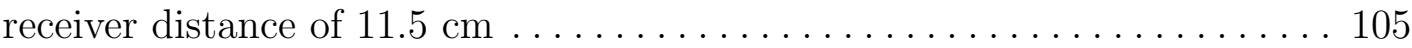
Figure 4.25 Enhanced BER based on 3ASK modulation for transmitter-

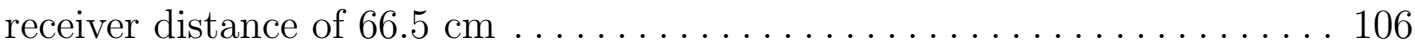
Figure 4.26 Enhanced BER based on 5ASK modulation for transmitter-

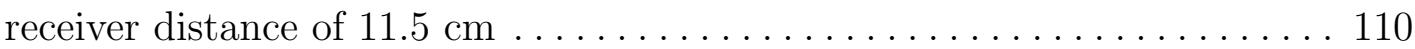
Figure 4.27 Enhanced BER based on 5ASK modulation for transmitter-

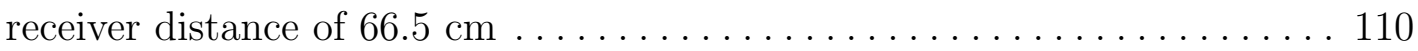
Figure 4.28 Enhanced 3ASK modulation data rate vs distance $(\mathrm{cm})$ for

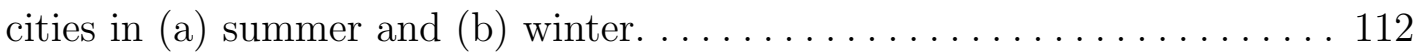
Figure 4.29 Enhanced 5ASK modulation data rate vs distance $(\mathrm{cm})$ for

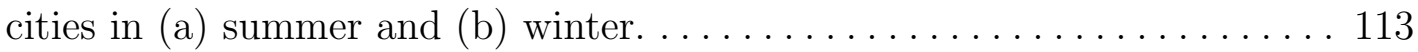

Figure 5.1 Atmospheric attenuation coefficient for multiple relative humidity levels and the three channels with relatively less attenuations. . . . . 115 Figure 5.2 Received power of 220-255 GHz (channel A), 660-695 GHz (channel B) and 855-890 GHz (channel C) using omni-directional anten-

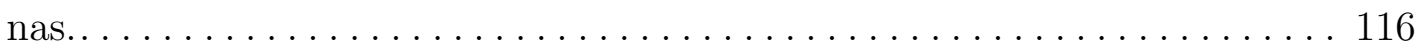

Figure 5.3 Received power of $220-255 \mathrm{GHz}$ (channel A) using omni-

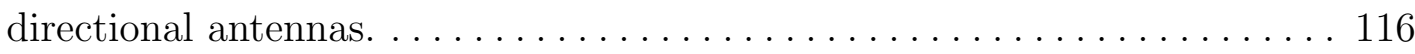

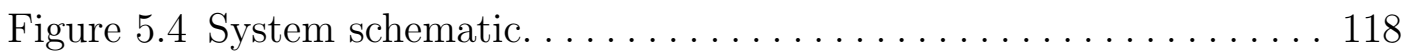


Figure 5.5 Time slots assigned to each user in SCA.. . . . . . . . . . . 118

Figure 5.6 Bit error rate (BER) vs received $\mathrm{E}_{\mathrm{b}} / \mathrm{N}_{0}$ for modulation meth-

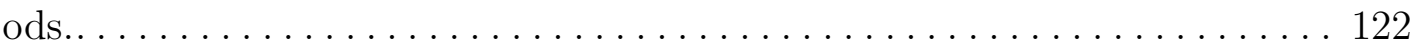

Figure 5.7 Bit error rate vs distance $(\mathrm{cm})$ for modulation methods using channel A for transmission.. . . . . . . . . . . . . . . . . . . . . . . . . 122

Figure 5.8 Bit error rate vs distance $(\mathrm{cm})$ for modulation methods using channel B for transmission... . . . . . . . . . . . . . . . . . . . . . . . . . . 123

Figure 5.9 Bit error rate vs distance $(\mathrm{cm})$ for modulation methods using channel $\mathrm{C}$ for transmission.. . . . . . . . . . . . . . . . . . . . . . . . . . . . 123

Figure 5.10 Data rate (bps) vs distance (cm) for modulation methods using channel A. . . . . . . . . . . . . . . . . . . . . 124

Figure 5.11 Data rate (bps) vs distance $(\mathrm{cm})$ for modulation methods using channel B. . . . . . . . . . . . . . . . . . . . . . . . . . . 124

Figure 5.12 Data rate (bps) vs distance $(\mathrm{cm})$ for modulation methods

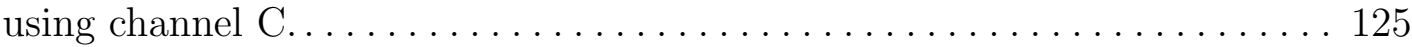

Figure 5.13 The assigned time percentage to each user when channel A

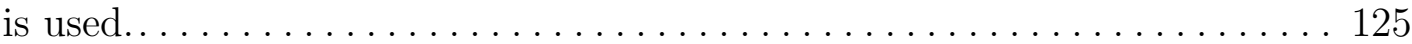

Figure 5.14 The assigned time percentage to each user when channel B

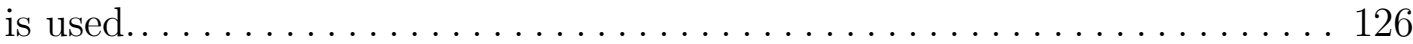

Figure 5.15 The assigned time percentage to each user when channel $\mathrm{C}$

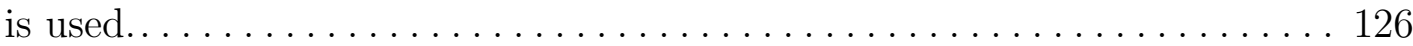

Figure 5.16 SCA downlink data rate when TAP uses channel A, B, or C.. 127 Figure 5.17 MCA downlink data rate.. . . . . . . . . . . . . . . 127

Figure 5.18 The percentage of time that TAP assigns different channels to each user in MCA.. . . . . . . . . . . . . . . . . . . . . . . . . . . 128

Figure 5.19 Transmitted power vs. coverable transmitter-receiver distance when BPSK is used on channel A and BER $\leq 10^{-4} \ldots \ldots \ldots \ldots \ldots 129$

Figure 6.1 Block diagram of optoelectronic technique that generates $\mathrm{THz}-$

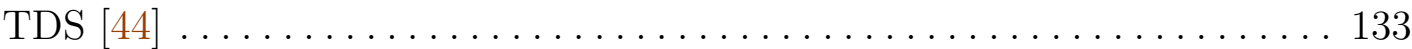

Figure 6.2 Block diagram of optoelectronic technique that generates CW signal with two tunable lasers. . . . . . . . . . . . . . . . . . . . 133

Figure 6.3 A power comparison between the signals generated based on THz-TDS and Heterodyne systems $[40] \ldots \ldots \ldots \ldots \ldots \ldots \ldots$. . . . . . . . . 134

Figure 6.4 A transmitter with microwave frequency multiplier compo-

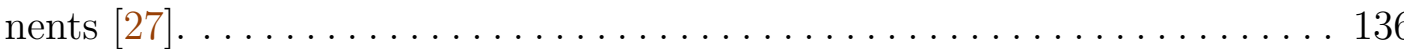


Figure 6.5 Typical configuration of a receiver based on heterodyne tech-

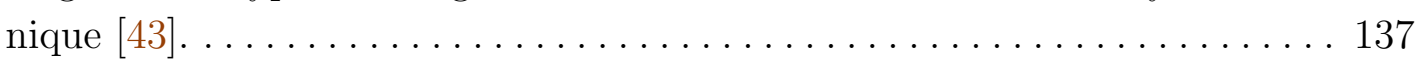
Figure 6.6 Typical configuration of a receiver based on direct detection

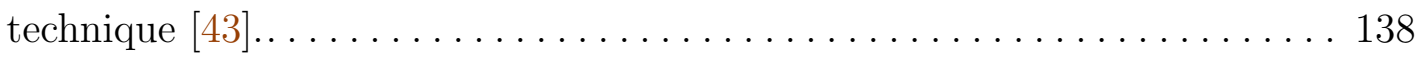


Chapter 1

INTRODUCTION

\section{WHY DO WE NEED HIGHER DATA RATES}

The demand for higher data rates is ever increasing and is unlikely to stabilize anytime soon. This trend is driven by increasing number of deployed devices providing content-rich data to users and the demand from users for greater download speeds, anywhere and anytime. For instance, the Japanese government estimated that the annual growth rate of mobile traffic in Japan is around $71 \%$; this rate for United states is estimated to be around 117\% [63]. According to Edholm's law of bandwidth [10], the demand for bandwidth in wireless communications has doubled every 18 months over the last 25 years. Therefore, larger bandwidths and tens of Gbps data rates will be required by 2020 [16, 33].

Aside from the typical mobile applications, newer uses of high-speed wireless links also require very high data rates. For example, uncompressed high-definition TV (HDTV) has a bit rate of over 1.5 Gbps when transmitted from the DVD to the TV set. Ultrahigh definition TV (UHD) or 4k-TV has a 16 times increased resolution compared to HDTV. Therefore, data rates as high as 24 Gbps will be required for their transmission. For users in cities where the cost of optical cables is high and for the last mile problem, such as the last mile transmission of multiple channel HDTV, we need to have wireless data rates comparable to data rates in 
wireline communications [16]. In addition, board to board communication inside a computer [33], downloading high definition movies from a kiosk [37], transferring a large amount of data between mobile terminals and storage devices wirelessly, and connecting a laptop wirelessly to the TV instead of using HDMI cables also need higher wireless data rates than what is available today.

One of the primary limitations in delivering high data rates is that most of the bandwidth in the microwave range $(300 \mathrm{MHz}-100 \mathrm{GHz})$ is already reserved for some applications and only limited bandwidth can be used for general communication purposes. Researchers have used advanced modulation methods and multipleinput and multiple-output (MIMO) to better utilize this available bandwidth and achieve higher data rates. They utilize advanced modulation methods to increase the spectral utilization efficiency (more bits per second per hertz of bandwidth). These methods increase data rates, enhance sharing of a given band of frequencies, and enable higher amounts of frequency reuse within the same volume of space. In MIMO, multiple antennas are used at both the transmitter and the receiver to improve coverage, reliability, and capacity of transmission. MIMO is used in many wireless communication standards such as IEEE 802.11n and IEEE 802.11ac (WiFi), and 4G. Even though by utilizing these techniques higher data rates have been achieved, based on Shannon's channel capacity formula there is an upper bound on the achievable data rate for a given bandwidth, and user demand has already exceeded this capacity [16].

In order to achieve higher data rates it is necessary to use a frequency range that has greater available bandwidth. One option is the Terahertz $(\mathrm{THz})$ spectrum that has higher frequency than the microwave domain and has high available bandwidth that is not reserved for any specific application. Because of that, the $\mathrm{THz}$ domain has gained a great deal of attention over the past few years as a technology that 


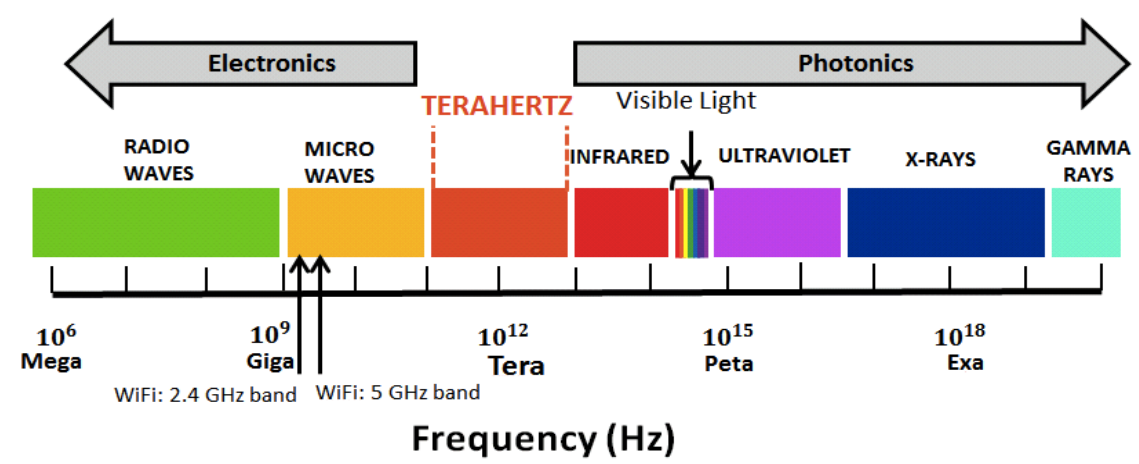

Figure 1.1: Terahertz spectrum sits between microwave and infrared regions.

can achieve the required data rate for future applications. To explore the feasibility of the $\mathrm{THz}$ domain for wireless communication the IEEE 802.15 Terahertz Interest Group was formed in 2008 [37].

\section{WHAT IS TERAHERTZ}

$\mathrm{THz}$ domain has a frequency range from $0.1 \mathrm{THz}$ to $10 \mathrm{THz}(30 \mu \mathrm{m}$ to $3 \mathrm{~mm}$ wavelength) and is located between the microwave and infrared light in the electromagnetic spectrum. Figure 1.1 shows the $\mathrm{THz}$ domain as well as other domains in the electromagnetic spectrum.

THz signals have two significant advantages over microwaves:

- $\mathrm{THz}$ domain has a large available bandwidth that in theory could deliver multiple Terabits/s (Tbps) data rates.

- THz signals are attenuated by atmospheric molecules. Therefore, THz communication is relatively secure and can be used in applications where security is a must [63].

$\mathrm{THz}$ signals also have advantages over Infrared (IR) signals. For example, 
- $\mathrm{THz}$ signals have lower frequency than IR signals. Therefore, they are less affected by atmospheric attenuation and as a result they propagate further.

- As $\mathrm{THz}$ signals have longer wavelengths than IR signals, they are safer to our health. For example, because of health hazards, the transmission power of IR signals is limited to eye-safe power levels [39].

- Because of limitations, such as poor sensitivity of incoherent receivers, high defuse reflection losses and high ambient light noise of IR signals, only Mbps data rates have been achieved using IR frequencies [33, 45].

\section{PRACTICAL PROBLEMS OF USING TERAHERTZ}

For many years, due to the lack of appropriate signal generators, transmitters and receivers, $\mathrm{THz}$ signals were only used in astronomy and other related fields [68]. However, the invention of new and improved $\mathrm{THz}$ transmitters and detectors, such as femtosecond lasers and photoconductive antennas, has made it possible to use $\mathrm{THz}$ signals in wireless communication [32, 42, 58, 63] as well as some other fields, such as explosive and concealed weapon detection $[7,17]$ and pharmaceutical quality control [72].

In the past few years, various kinds of devices have been reported that can be used at the transmitter and receiver front ends of $\mathrm{THz}$ communications. However, $\mathrm{THz}$ communication is in early stages of its development and there are still significant technical challenges. For example, electronic devices based on compound semiconductor transistors have been considered one of the best candidates for generating and detecting $\mathrm{THz}$ signals with output power in the range of $\mathrm{mW}$, but they only work for frequencies up to $\sim 350 \mathrm{GHz}$. For frequencies above 350 $\mathrm{GHz}$, photonic devices, such as photodiodes, that have been originally developed 
for fiber-optic communications need to be used. One limitation of using photonic technology is that the generated output power is low - in the range of $\mu \mathrm{W}$. Therefore, due to limited output power and lack of having sensitive enough transmitters and receivers at high frequencies, most of the experimental demonstrations have been conducted at frequencies below $350 \mathrm{GHz}$ [63].

The higher frequency means smaller wavelength. $\mathrm{THz}$ signals can penetrate some non-conduction materials such as paper, wood, and plastic. However, their penetration depth is less than microwave signals due to their smaller wavelength. $\mathrm{THz}$ signals have limited penetration through fog and clouds and they cannot go through liquid water and metallic objects. Also, the ratio between the size of objects and the signal becomes larger in $\mathrm{THz}$ range as opposed to microwave range. In this case, the roughness of surfaces cause an additional attenuation as the signal scatters more and the reflected signal becomes very weak [37, 48]. Figure 1.2 shows the effect of a rough surface on the reflected signal. As we see, the rough surface diffuses the signal and causes a weak reflection.

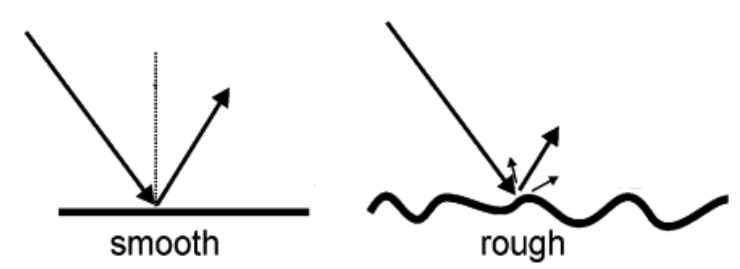

Figure 1.2: The effect of a smooth (on the left) and a rough (on the right) surface on the reflected signal [48].

The other challenge in using THz communication is attenuation. Due to dissipation, the signal's energy attenuates as it travels the distance between the transmitter and the receiver. This attenuation is given by Friis transmission formula 
as free-space loss $\left(\frac{4 \pi f d}{c}\right)^{2}$, where, $c$ is the speed of light, $f$ is the frequency of the electromagnetic signal, and $d$ is the transmitter-receiver distance [33]. According to this formula, the high frequency of $\mathrm{THz}$ range, $f$, corresponds to high free-space loss.

Further, molecules in the atmosphere, especially water molecules, absorb the signal energy at some frequencies. Figure 1.3 shows the overall attenuation as a function of distance and frequency in the range of $0-1 \mathrm{THz}$ and Figure 1.4 shows the $\mathrm{THz}$ atmospheric transmission for frequencies up to $5 \mathrm{THz}$. As Figure 1.3 shows, the attenuation increases at higher frequencies and with longer distances.

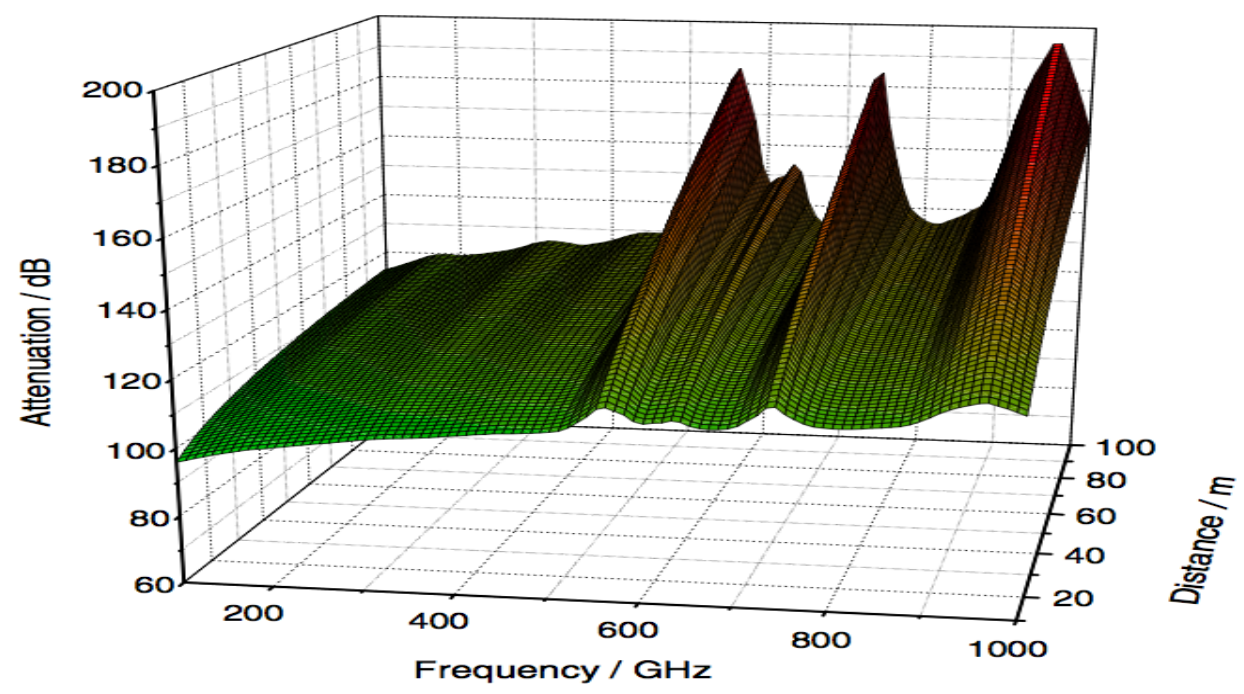

Figure 1.3: Atmospheric attenuation as a function of frequency and distance in the range of $0-1 \mathrm{THz}[33]$. 


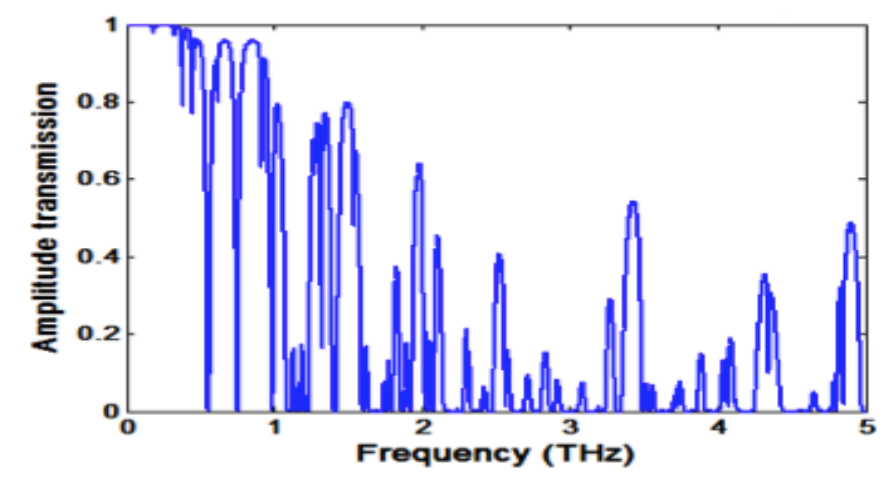

Figure 1.4: Atmospheric transmission of $\mathrm{THz}$ signal within $0-5 \mathrm{THz}$ [71].

\section{PROPOSED RESEARCH}

Thesis statement: Can we develop techniques that can lead to tens of Gbps data rates using THz signals?

Currently due to high atmospheric attenuation and the limited output power, pulsed $\mathrm{THz}$ signals have been only used in nano-network communications. Also, there are no media access control (MAC) protocols for sharing THz signals among different users.

In this research we will develop a technique to address the thesis question using pulsed $\mathrm{THz}$ and continuous waves $(\mathrm{CW})$. In the case of pulsed $\mathrm{THz}$, we will use the large bandwidth of each $\mathrm{THz}$ pulse to modulate a large number of data bits. We will develop techniques to adapt our method such that atmospheric attenuation and transmitter-receiver distance do not affect the bit error rate (BER) at the receiver drastically and we achieve better BER under higher humidity levels and longer transmitter-receiver distances. We will also develop adaptation algorithms to be used among multiple users with the goal of maintaining fairness and achieving high data rates. 
The research will rely on detailed channel measurements, using the Picometrix T-Ray 4000 system, and analysis for the case when using a collimated lens as well as the case where the transmitter and receiver have no lens. After we fully understand the channel, we will use Matlab simulations to develop and analyze new communication methods.

\section{MAJOR RESEARCH CONTRIBUTIONS}

The major outcomes of this research can be summarized as follows:

- Detailed channel characterization using collimating lens for directional channels.

- Detailed channel characterization using omni-directional antennas for omnidirectional channels.

- Analysis of pulsed $\mathrm{THz}$ signals in wireless communications for the first time in channels other than nano-networks.

- Develop techniques to get better BER in the case of high humidity and long transmitter-receiver distances.

- Study the usage of continuous wave $\mathrm{THz}$ wireless communications in omnidirectional channels.

- Develop rate adaptation algorithms to be used in THz systems.

\section{THESIS ORGANIZATION}

In the next chapter we summarize the technical background related to this thesis. In Chapter 3 we provide indoor $\mathrm{THz}$ channel characterization to develop models 
to be used in Matlab simulations. Chapters 4 and 5 examine different modulation methods in directional and omni-directional $\mathrm{THz}$ channels, respectively, using the analysis from Chapter 3. Finally, a detailed literature review is provided in Chapter 6 followed by our conclusion in Chapter 7 . 
Chapter 2

\section{OVERVIEW OF COMMUNICATION SYSTEMS}

Communication systems are built to transmit information signals from a source, located at one point, to a destination, located at another point some distance away. A signal is defined as a single-valued function of time that conveys information. That is, every instant of time corresponds to a unique value of the signal. A discrete-time signal is defined only at discrete instants of time and are described as sequences of samples that may take on continuous values. When each sample of a discrete-time signal is quantized and then coded, the resultant signal is called a digital signal.

Communication systems consist of three main parts: transmitter, channel, and receiver. The transmitter processes the message signal into a suitable form for transmission over the channel (modulation). The channel provides a physical connection between the output of the transmitter and the input of the receiver. The function of the receiver is to process the received signal so that it can produce an estimate of the original message signal (detection or demodulation). Figure 2.1 shows a block diagram of the communication system.

In any communication system, there are two primary communication resources which are average transmitted power and channel bandwidth. The average transmitted power is the average power of the signal that is transmitted. The channel bandwidth is the range of frequencies that the channel can use for the transmission of signals. The objective in designing communication systems is to use these two 


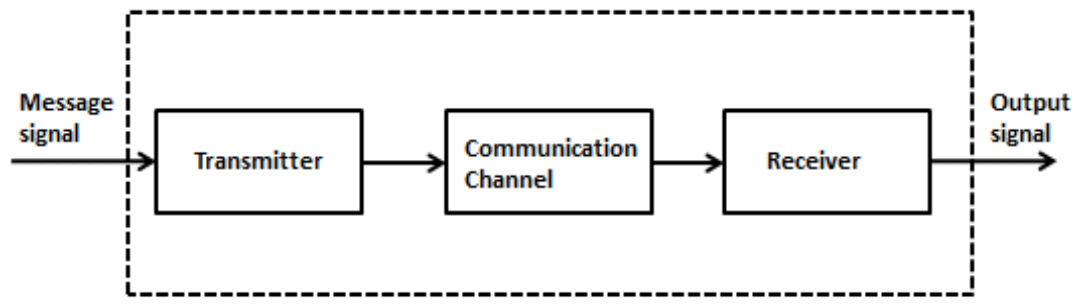

Figure 2.1: Block diagram of a communication system.

resources as efficiently as possible.

Transmitted power is important because it determines the signal to noise ratio (SNR) at the receiver. SNR determines the noise performance at the receiver and if the received signal has SNR below some level, the transmission of the signal is not considered satisfactory. Noise refers to unwanted electromagnetic signals that are present in every communication system. Noise limits the receiver's ability to correctly detect symbols due to the fact that noise masks the signal. Thermal noise is one of the natural sources of noise that is caused by the thermal motion of electrons in all dissipative components. We can describe the thermal noise as a zero-mean Gaussian random process. A Gaussian process $n(t)$ is a random function whose value $n$ at any time $t$ is characterized by the Gaussian probability density function that is as follows:

$$
p(n)=\frac{1}{\sigma \sqrt{2 \pi}} \mathrm{e}^{-\frac{1}{2}\left(\frac{n}{\sigma}\right)^{2}}
$$

Here $\sigma^{2}$ is the variance of $n$. We set $\sigma=1$ in the normalized Gaussian density function of a zero-mean process.

Thermal noise has the same power spectral density $(\mathrm{PSD})^{1}$ for all frequencies

\footnotetext{
${ }^{1}$ Note that power spectral density (PSD) is a function of frequency and shows the strength of the signal's energy at each frequency.
} 
of interest in all communication systems. Therefore, a model for thermal noise assumes that the power spectral density $G_{n}(f)$ of noise is the same for all frequencies and can be found from the following equation:

$$
G_{n}(f)=\frac{N_{0}}{2}
$$

The factor 2 indicates that $G_{n}(f)$ is a two-sided power spectral density and $N_{0}$ is noise power spectral density in Joules, given by $N_{0}=k T$, where $k$ is Boltzmann's constant, $1.38 \times 10^{-23}$ Joules/Kelvin, and $T$ is the receiver system noise temperature in Kelvin.

Error performance of a channel can be stated as energy per bit to noise power spectral density ratio $\left(E_{b} / N_{0}\right)$ of the channel and is defined as follows:

$$
\frac{E_{b}}{N_{0}}=\frac{S T_{b}}{N / B}=\frac{S}{N}\left(\frac{B}{R_{b}}\right)
$$

where $E_{b}$ is bit energy that is the signal power $S$ times the bit time $T_{b}, R_{b}$ is bit rate which is the reciprocal of the bit time $T_{b}$, and $N_{0}$ can be written as noise power $N$ divided by bandwidth $B$. The dimensionless $E_{b} / N_{0}$ is a standard quality measure for digital communications system performance. The channel with a smaller $E_{b} / N_{0}$ for the same data rate and error probability indicates that it has a more efficient detection process than a channel with higher $E_{b} / N_{0}$.

SNR is degraded if the signal power is decreased and/or if the noise power is increased. One of the reasons that can decrease the signal power is loss. Losses in the channel occur when the receiver does not receive some portions of the signal. That is portions of the signal are absorbed, diffracted, or scattered when the signal propagates through the channel.

The number of received bits at the receiver that have been altered and are not detected correctly gives us the number of bit errors. Bit error ratio (BER) is the number of bit errors divided by the total number of bits that are transferred. 
BER is dimensionless and usually is measured in percentage. Bit error probability, $P_{B}$, is the expectation of the BER. The BER of a system may be improved by transmitting a stronger signal at the transmitter.

In addition to the signal's transmission power, bandwidth of the channel is also important in determining data rate. Based on Shannon's capacity theorem we have:

$$
C=B \log _{2}\left(1+\frac{S}{N}\right)
$$

where $B$ is the bandwidth of the channel in $\mathrm{Hz}$, and $C$ is the capacity in bits/s (bps). The channel capacity is the maximum bit rate at which information may be transmitted without any errors through the channel. This equation shows that the channel capacity depends on the bandwidth and the SNR of the transmission system.

\section{FOURIER TRANSFORM}

Fourier transform is a mathematical function of time into the frequency components that make up the signal. That is the Fourier transform of a function of time is a complex valued function of frequency. The absolute value of each fre-

quency component represents the amount of that frequency present in the original function. The complex argument is the phase offset of the basic sinusoid in that frequency.

The Fourier transform of the signal $f(t)$ is equal to the following.

$$
F(\omega)=\int_{-\infty}^{\infty} f(t) e^{-j \omega t} d t
$$

where, $F(\omega)$ is a complex-valued function of angular frequency, $\omega=2 \pi f$ and $f$ corresponds to frequency. Inverse Fourier transform is calculated as:

$$
f(t)=\frac{1}{2 \pi} \int_{-\infty}^{\infty} F(\omega) e^{j \omega t} d \omega
$$


The continuous Fourier transform converts a signal in time-domain of infinite duration into a continuous spectrum composed of an infinite number of sinusoids. However, we usually deal with discretely sampled signals with constant intervals that are of finite duration or periodic. In this case, only a finite number of sinusoids is needed and the discrete Fourier transform (DFT) is appropriate. For a discrete signal $x[n]$, we calculate the Fourier transform as follows:

$$
X[k]=\sum_{n=0}^{N-1} x[n] e^{-j 2 \pi k n / N}
$$

The following is the inverse Fourier transform:

$$
x[n]=\frac{1}{N} \sum_{k=0}^{N-1} X[k] e^{j 2 \pi k n / N}
$$

\section{ENERGY AND POWER OF THE SIGNAL}

Energy, $E$, of a continuous signal in time domain, $x(t)$, is defined as follows:

$$
E=\int_{-\infty}^{\infty}|x(t)|^{2} d t
$$

According to Parseval's theorem, the Fourier transform is unitary. That is, the energy of the signal in time domain is equal to its energy in frequency domain. Therefore:

$$
\int_{-\infty}^{\infty}|x(t)|^{2} d t=\int_{-\infty}^{\infty}|X(f)|^{2} d f
$$

where, $x(t)$ is the continuous signal in time domain and $X(f)$ is the correspond-

ing frequency domain (spectrum) of the signal. For the discrete signal $x[n]$, the Parseval relation is changed as follows.

$$
\sum_{n=0}^{N-1}|x[n]|^{2}=\frac{1}{N} \sum_{k=0}^{N-1}|X[k]|^{2}
$$


where, $x[n]$ is the sampled signal in time domain and $X[k]$ is the frequency domain of the corresponding signal. Note that, the length of both $x[n]$ and $X[k]$ is the same and is equal to $N$ in this equation.

Power is the amount of energy consumed per unit time. The following equation shows the power for continuous signal $x(t)$ that is extended infinitely in time domain.

$$
P(t)=\lim _{T \rightarrow \infty} \frac{1}{T} \int_{-T / 2}^{T / 2}|x(t)|^{2} d t
$$

Here, $T$ is the time period of the signal. In case of discrete signal $x[n]$ that is extended infinitely in time, the power equation is as follows.

$$
P(t)=\lim _{N \rightarrow \infty} \frac{1}{2 N+1} \sum_{n=-N}^{N}|x[n]|^{2}=\lim _{N \rightarrow \infty} \frac{1}{N} \sum_{n=0}^{N-1}|x[n]|^{2}
$$

From (2.11), we calculate the power of the corresponding frequency domain signal $X[k]$ from the following equation.

$$
P(f)=\lim _{N \rightarrow \infty} \frac{1}{N^{2}} \sum_{k=0}^{N-1}|X[k]|^{2}
$$

Note that a signal that has finite energy and zero average power is called an energy signal. Also, a signal with infinite energy and finite average power is called a power signal. As a result, energy signals are time limited, whereas power signals can exist over infinite time. Moreover non-periodic signals are energy signals while power signals are always periodic.

Energy spectral density (ESD) measures the distribution of signal energy over frequency and has the unit of $\mathrm{J} / \mathrm{Hz}$. Power spectral density (PSD) measures the distribution of signal power over frequency domain and has the unit of $\mathrm{W} / \mathrm{Hz}$.

\section{THE CHANNEL}

The electromagnetic path or the propagation medium that connects the transmitter to the receiver is called the channel. The communication channel might consist 
of wires, fiber optics and in the case of wireless communication, waveguides, atmosphere, or free space. The free space concept assumes a channel that does not cause any absorption, reflection, refraction, or diffraction of the signal. In a free space channel the energy of the signal is a function of distance. In this ideal channel the received power at the receiver is attenuated by a factor $L_{s}(d)$, which is called path loss or free space loss. Path loss is computed as follows:

$$
L_{s}(d)=\left(\frac{4 \pi d}{\lambda}\right)^{2}
$$

where $d$ is the transmitter-receiver distance, and $\lambda$ is the wavelength of the propagating signal.

The signal power that is received by one antenna under ideal conditions can be modeled by Friis transmission equation as below:

$$
\frac{P_{r}}{P_{t}}=\frac{G_{t} G_{r}}{L_{s}(d)}=G_{t} G_{r}\left(\frac{\lambda}{4 \pi d}\right)^{2}
$$

where $P_{r}$ is the received power at the receiver, $P_{t}$ is the transmitted signal's power, $L_{s}(d)$ is the free space path loss from $(2.15), G_{t}$ and $G_{r}$ are the antenna gain in the transmitter and receiver, respectively. We can find the antenna gain from the following equation:

$$
G=\frac{4 \pi A}{\lambda^{2}}
$$

Here $A$ is the aperture size of the antenna. As the equation shows, for a given antenna gain, as $\lambda$ is increased we need to have an antenna with a bigger aperture size.

In practice, propagation through the channels that take place in the atmosphere and near the ground is not ideal and results in absorption, reflection, diffraction and scattering. Some molecules in the atmosphere, such as oxygen and water vapor, absorb some of the signal's energy as it propagates through the channel. 
As a result of absorption, the molecules radiate energy that causes noise in the channel. When a signal hits a smooth surface that has relatively larger dimension relative to the wavelength of the signal, $\lambda$, reflection occurs. Diffraction happens in non line-of-sight (NLOS) channels when an object with dense body, which has large dimensions relative to $\lambda$, is placed in the propagation path of the signal. As a result, secondary waves are formed behind the obstructing body and diffraction occurs. When the signal hits a large, rough surface or a surface that has a length smaller or in the range of $\lambda$, the signal is scattered and its energy spreads out in all directions.

\subsubsection{Channel Modeling}

The first step to designing a wireless communication system is to develop and characterize the model of the channel that properly describes the environment under which we are going to use our system. For that we need to study the impulse response of the channel that mimics the applications environment. Figure 2.2 shows a linear system that models the communication channel. The input

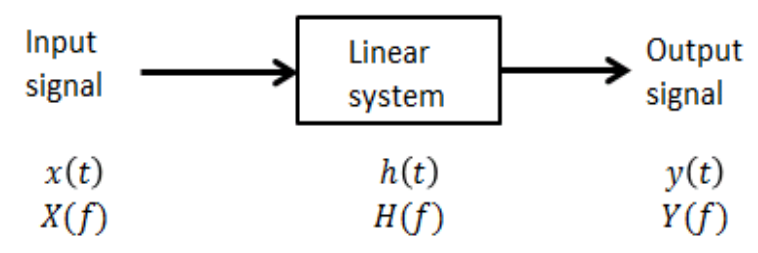

Figure 2.2: Linear system.

signal to a linear system can be considered in time domain as $x(t)$ or in frequency domain as $X(f)$. The transmitted signal goes through the linear and time invariant system and results in output signal $y(t)$ or $Y(f)$ in time domain and frequency domain, respectively. We characterize the channel with impulse response function, 
$h(t)$, in time domain. That is the response, or output, of the system when the input signal is a unit impulse $\delta(t)$. That is:

$$
\text { if } x(t)=\delta(t) \quad \text { then } h(t)=y(t)
$$

Figure 2.3 shows the unit impulse function. As the figure shows, the unit impulse

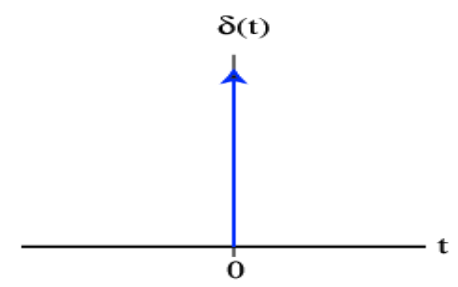

Figure 2.3: Unit impulse function.

function or Dirac delta function $\delta(t)$ is a large amplitude pulse with zero pulse width and unity area under the pulse that is concentrated at the point where its argument is zero. It is defined by the following relationship:

$$
\begin{aligned}
& \int_{-\infty}^{\infty} \delta(t) d t=1 \\
& \delta(t)=0 \text { for } t \neq 0 \\
& \delta(t) \text { is unbounded at } t=0
\end{aligned}
$$

The impulse response of the channel relates to the transmitted and received signals as follows:

$$
y(t)=x(t) \star h(t)=\int_{-\infty}^{\infty} x(\tau) h(t-\tau) d \tau
$$

Where $\star$ indicates the convolution operation. $h(t)$ encapsulates all the environmental impairments suffered by the signal $x(t)$ as it travels to the receiver. Since the communication system is linear time-invariant, $h^{-1}(t)$ exists and is unique. Thus, 
if the receiver knows $h(t)$, it can retrieve the signal $x(t)$ exactly by computing,

$$
x(t)=y(t) * h^{-1}(t)
$$

Unfortunately, in reality $h(t)$ is not known accurately and it is time varying. Typ-

ically, the receiver estimates the channel impulse response $\hat{h}(t)$ and computes,

$$
\hat{x}(t)=y(t) \star \hat{h}^{-1}(t)
$$

The channel estimator $\hat{h}(t)$ is constructed by requiring the transmitter to transmit well known symbols periodically. In that case, $x(t)$ is known at the receiver as is $y(t)$ and thus,

$$
\hat{h}(t)=y(t) \star x^{-1}(t)
$$

and the channel estimator in frequency domain, $H(f)$, is constructed as follows:

$$
\hat{H}(f)=\frac{Y(f)}{X(f)}
$$

\section{PULSE SHAPING (FILTER)}

A filter is a device that is used to limit the spectrum of a signal to some specified band of frequencies. For example, the 802.11g standard that is used in WiFi limits transmitters to use $20 \mathrm{MHz}$ bandwidth at $2.4 \mathrm{GHz}$ frequency. Therefore, filters should be used in transmitters to limit the transmission bandwidth.

The frequency response of a filter is characterized by a passband and a stop band. All the frequencies inside the passband are transmitted, whereas the frequencies in the stop band are rejected. Filters can be used in both transmitters and receivers. The filters that are used in receivers are also referred to as equalizing filters. 


\subsubsection{Raised Cosine Filter}

Raised cosine filter is a low pass filter and is frequently used in receivers. The frequency-domain of the filter can be expressed as below:

$$
C(f)= \begin{cases}T, & \text { for }|f| \leq \frac{1-\beta}{2 T} \\ \frac{T}{2}\left[1+\cos \left(\frac{\pi T}{\beta}\left[|f|-\frac{1-\beta}{2 T}\right]\right)\right], & \text { for } \frac{1-\beta}{2 T}<|f| \leq \frac{1+\beta}{2 T} \\ 0, & \text { otherwise }\end{cases}
$$

Where, $\beta$ is called the roll-off factor and $0 \leq \beta \leq 1$, and $T$ is the symbol time. Figure 2.4 shows the filter in frequency domain. As the figure shows, the filter

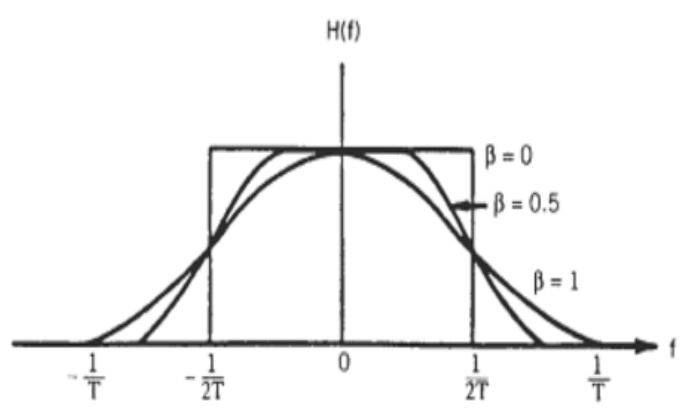

Figure 2.4: Raised cosine filter [46, p.334].

with $\beta=0$ corresponds to an ideal filter.

\subsubsection{Matched Filter}

Matched filter is used to detect the received signal at the receiver. It is a linear filter that is designed to provide the maximum signal to noise ratio for a given transmitted signal. Equation (2.25) shows the basic property of a matched filter:

$$
m(t)= \begin{cases}s(T-t) & 0 \leq t \leq T \\ 0 & \text { elsewhere }\end{cases}
$$


From (2.25) a matched filter is the mirror image of the signal, $s(t)$, that is delayed by the symbol time duration $T$.

The output of the matched filter in time domain, $z(t)$, is defined as the convolution of the filter, $m(t)$, with the received signal at the receiver, $r(t)$, that is:

$$
z(t)=r(t) \star m(t)=\int_{0}^{t} r(\tau) m(t-\tau) d \tau
$$

By substituting $m(t)$ from (2.25) into (2.26) we get the following equation:

$$
z(T)=\int_{0}^{T} r(\tau) s(\tau) d \tau
$$

As we know, the product integration of the received signal $r(t)$ with a replica of the transmitted signal $s(t)$ over one symbol interval is the correlation of $r(t)$ with $s(t)$. This technique is used in receivers to detect the received signal. That is, the received signal $r(t)$ is correlated with all signal prototypes $s_{i}(t)$ that are available at the receiver. The prototype $s_{i}(t)$ that yields the maximum output $z_{i}(T)$ is the signal that matches $r(t)$ better than other prototypes and is detected as the transmitted signal.

\section{CONTINUOUS WAVE MODULATION METHODS}

Communication systems can send the information signal from the transmitter to the receiver without changing the frequency of the signal, or they may shift the frequency of the signal to a higher frequency and then transmit it. The former is called baseband transmission while the latter is called bandpass transmission. In the case of bandpass transmission, the signal is shifted back to its original frequency at the receiver prior to detection.

The process of transforming digital symbols into waveforms that are compatible with the characteristics of the channel is called digital modulation. In the case of 
baseband modulation, the waveform that is used to send data usually takes the form of shaped pulses. However, in the case of bandpass modulation a sinusoid carrier wave is used to transmit data. The reason why carriers are used to send data can be explained as follows.

We use antennas in our communication channels to send information. As we explained in subsection 3, the size of the antenna depends on the frequency of the signal. As an example, the size of an antenna is typically $\frac{c}{4 f}$ in cellular networks, where $c$ is the speed of light and is equal to $3 \times 10^{8} \mathrm{~m} / \mathrm{s}$ and $f$ is the frequency of our signal. If the signal has the frequency of $3000 \mathrm{~Hz}$, we need an antenna with the diameter of 15 miles to send the signal without using a sinusoid carrier. However, if we use a sinusoid carrier and use a higher frequency of $900 \mathrm{MHz}$ to send our signal, we need an antenna with diameter of $8 \mathrm{~cm}$. Therefore, using bandpass modulation is an important step in communication channels. The process of shifting the baseband signal to bandpass frequency is called modulation and the process of shifting the bandpass signal to baseband frequency at the receiver is known as demodulation. In the modulation process, one or more characteristics of the carrier - amplitude, phase, or frequency — is changed according to the information signal — and are called amplitude modulation, phase modulation, and frequency modulation, respectively.

The general form of the carrier is as follows:

$$
s(t)=A(t) \cos \theta(t)
$$

Where, $A(t)$ is the time varying amplitude and $\theta(t)$ is the time varying angle of the carrier. Also, we usually write $\theta(t)=\omega_{0}(t)+\phi(t)$. Therefore, $(2.28)$ is changed to:

$$
s(t)=A(t) \cos \left[\omega_{0}(t)+\phi(t)\right]
$$


Here, $\omega_{0}$ is the radian frequency of the carrier and $\phi(t)$ is the phase. We should note that $\omega_{0}=2 \pi f$ where $f$ is the frequency in Hz. The sinusoid carrier can also be written in its complex form, from Euler's theorem, as follows:

$$
e^{j \omega_{0} t}=\cos \omega_{0} t+j \sin \omega_{0} t
$$

Where, $\cos \omega_{0} t$ is called the in-phase (real) and $\sin \omega_{0} t$ is called the quadrature (imaginary) parts of the carrier wave. The in-phase and quadrature parts are orthogonal and are called $I$ and $Q$, respectively.

When the signal arrives at the receiver, it needs to extract the information signal. As noted, the information signal may be represented in the phase, amplitude or frequency of the carrier. If the information is in the phase, then the receiver needs to be able to tell different phase shifts (one per information symbol) apart. This process has been implemented in two ways - coherent and non-coherent detection. Coherent detection is the case when the receiver uses a phase reference to exploit knowledge of the carrier phase to detect the signal. In coherent detection, the receiver has a prototype of each possible arriving signal. To detect the symbol, the receiver correlates, multiplies and integrates the incoming signal with the prototypes that it has. Phase shift keying (PSK) is an example of modulation that requires coherent detection. When the receiver does not require phase information of the received signal we call it non-coherent detection. Differential phase shift keying (DPSK) is an example of non-coherent modulation. In DPSK the phase information of the prior symbol is used as a phase reference for the phase detection of the current symbol.

\subsubsection{Phase Shift Keying (PSK)}

Phase Shift Keying (PSK) is one the modulation methods that is widely used in

communication systems today. The general form of PSK modulation is written as 
follows:

$$
\begin{gathered}
s_{i}(t)=\sqrt{\frac{2 E}{T}} \cos \phi_{i}(t) \cos (2 \pi f t)+\sqrt{\frac{2 E}{T}} \sin \phi_{i}(t) \sin (2 \pi f t), \\
0 \leq t \leq T \quad \text { and } \quad i=1, \cdots, M
\end{gathered}
$$

The phase term $\phi_{i}(t)$ will have $M$ different values, where $M$ is the modulation order:

$$
\phi_{i}(t)=\frac{2 \pi i}{M} \quad i=1, \cdots, M
$$

Here, each symbol corresponds to $\log _{2} M$ data bits. $T$ is the symbol time, $0 \leq t \leq T, E$ is the symbol energy, $f$ is the carrier frequency, and $s_{i}(t)$ corresponds to the signal that is generated from the $i$ th symbol.

The generated signal is then passed through the channel and it is received by the receiver. The following equation shows the received signal.

$$
\begin{aligned}
r(t)= & s_{i}(t) \star h(t)+n(t), \\
& 0 \leq t \leq T \text { and } i=1, \cdots, M
\end{aligned}
$$

Where, $h(t)$ is the impulse response of the channel, and $n(t)$ is the noise.

The received signal at the receiver, $r(t)$ is then demodulated as is shown in Fig. 2.5. In the figure, $\phi_{i}=\frac{2 \pi i}{M}$ is the original phase that corresponds to symbols $i=1, \cdots, M$ and $\hat{\phi}$ is the calculated received phase by the receiver. $\hat{s}_{i}(t)$ is the detected signal based on the estimated phase $\hat{\phi}$ at the receiver.

It should be noted that in $\sqrt{\frac{2}{T}} \sin (2 \pi f t)$ and $\sqrt{\frac{2}{T}} \cos (2 \pi f t)$, the amplitude $\sqrt{\frac{2}{T}}$ is chosen to normalize the expected output of the detector. 


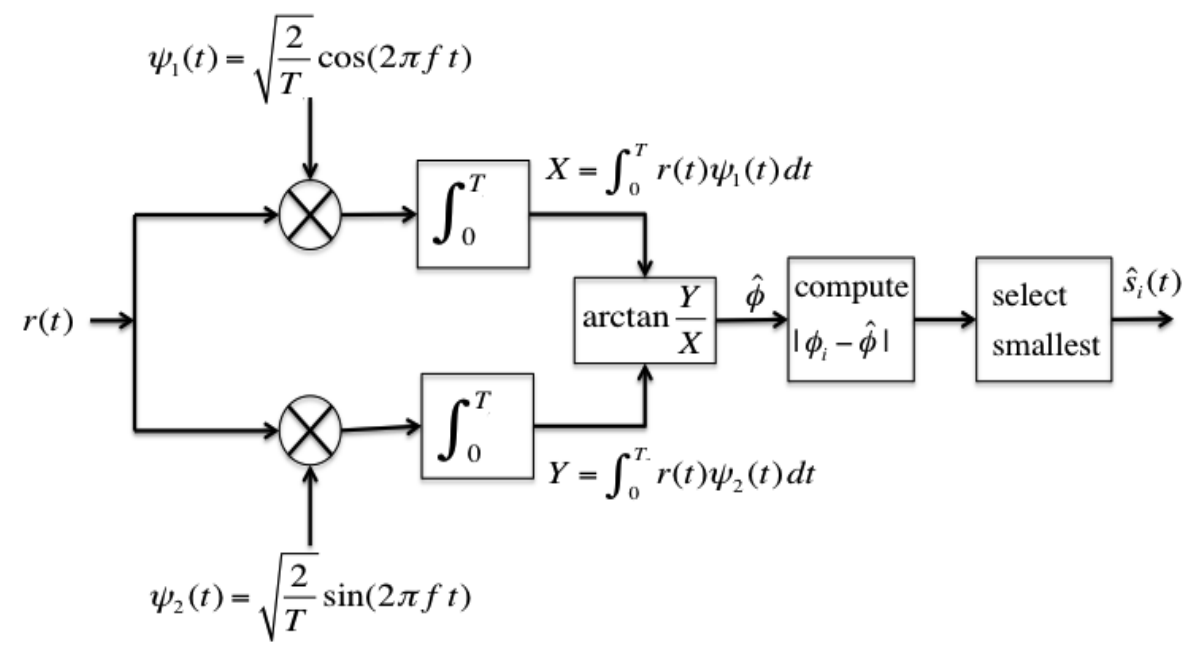

Figure 2.5: PSK receiver block diagram.

\subsubsection{Quadrature Amplitude Modulation (QAM)}

QAM modulation can be considered as a logical extension of PSK modulation with the addition of two independent amplitude-modulated carriers. In PSK modulation, the amplitude of the transmitted signal is constrained to remain constant. However, in QAM amplitude can also vary with the phase. Figure 2.6 shows the constellation diagram for 16QAM. A constellation is a representation of the possible symbols that may be selected by a given modulation scheme as points that show the corresponding in-phase and quadrature parts of a signal in the complex plane. 


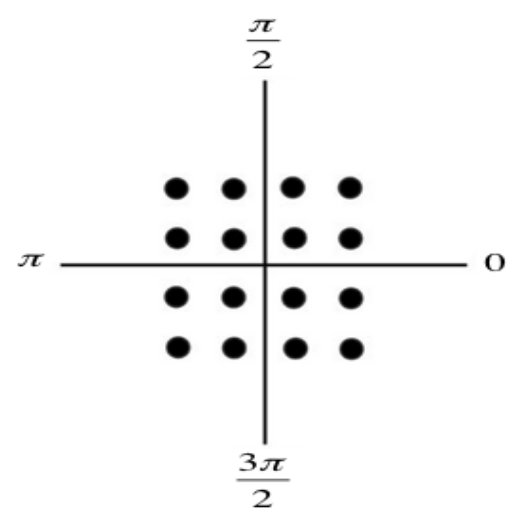

Figure 2.6: Constellation diagram 16QAM.

The general form of an M-ary QAM signal is as follows:

$$
\begin{aligned}
& s_{i}(t)= \sqrt{\frac{2 E_{\text {min }}}{T}} a_{i} \cos (2 \pi f t)+\sqrt{\frac{2 E_{\text {min }}}{T}} b_{i} \sin (2 \pi f t) \\
& 0 \leq t \leq T \quad i=1,2, \cdots, M
\end{aligned}
$$

Where $E_{\text {min }}$ is the energy of the signal when it has the lowest amplitude, $a_{i}$ and $b_{i}$ are a pair of integers that are chosen based on the location of the particular signal point on the constellation diagram.

The coordinates of the $i$ th signal are $a_{i} \sqrt{E_{\text {min }}}$ and $b_{i} \sqrt{E_{\text {min }}}$, where $\left(a_{i}, b_{i}\right)$ can be found from the following matrix:

$$
\left\{a_{i}, b_{i}\right\}=\left[\begin{array}{cccc}
(-L+1, L-1) & (-L+3, L-1) & \ldots & (L-1, L-1) \\
(-L+1, L-3) & (-L+3, L-3) & \ldots & (L-1, L-3) \\
\vdots & \vdots & \ddots & \vdots \\
(-L+1,-L+1) & (-L+3,-L+1) & \ldots & (L-1,-L+1)
\end{array}\right]
$$

Where $L=\sqrt{M}$ and $M$ is the modulation order. For example, for 16QAM with the 
constellation diagram shown in Figure 2.6, the corresponding matrix is as follows:

$$
\left\{a_{i}, b_{i}\right\}=\left[\begin{array}{cccc}
(-3,3) & (-1,3) & (1,3) & (3,3) \\
(-3,1) & (-1,1) & (1,1) & (3,1) \\
(-3,-1) & (-1,-1) & (1,-1) & (3,-1) \\
(-3,-3) & (-1,-3) & (1,-3) & (3,-3)
\end{array}\right]
$$

At the receiver, a matched filter is used to estimate the received $a_{i}$ and $b_{i}$. Figure 2.7 is the block diagram of the receiver.

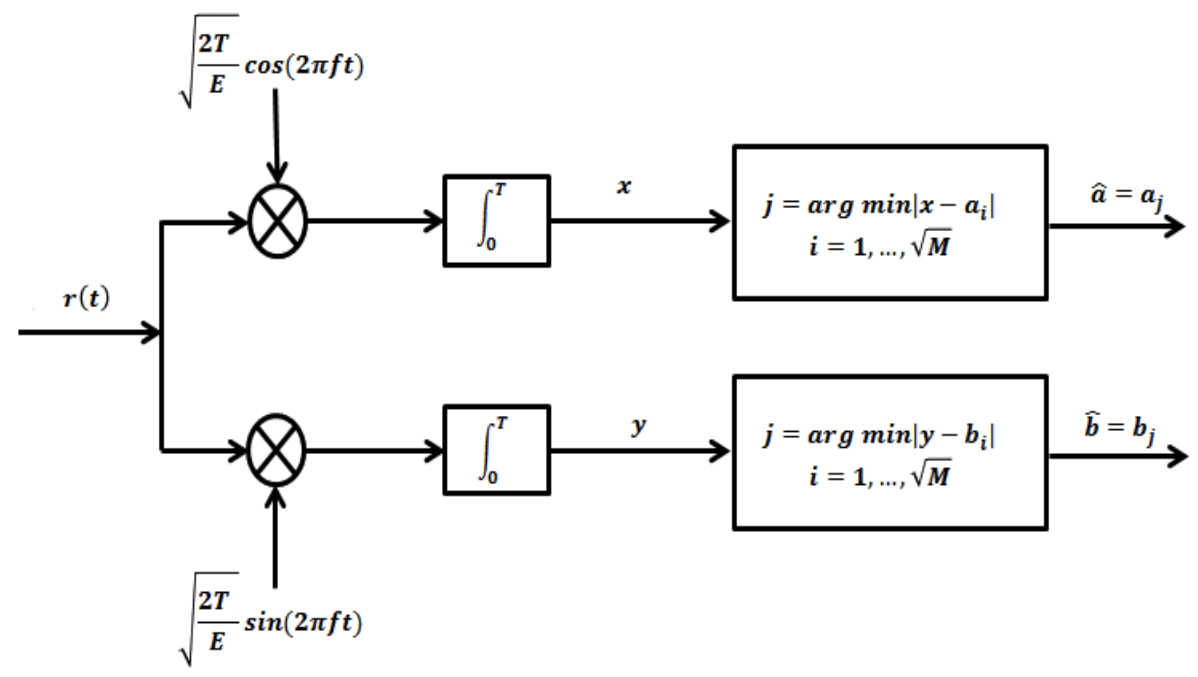

Figure 2.7: Block diagram of QAM receiver.

\section{PULSED SIGNAL MODULATION METHODS}

One method that can be used to transmit digital information from the transmitter to the receiver is to use pulsed signals. Pulsed signals are ultra short pulses in the time domain and as a result occupy a very wide bandwidth. 


\subsubsection{Amplitude Shift Keying (ASK)}

If we assume that $x(t)$ is a pulsed signal with amplitude 1 , the general form of a pulse signal with amplitude shift keying (ASK) modulation is as follows:

$$
\begin{aligned}
& s_{i}(t)=A_{i} x(t), \\
& 0 \leq t \leq T \text { and } i=1, \cdots, M,
\end{aligned}
$$

Where, $A_{i}$ is the amplitude of the $i$ th symbol, and $M$ is the modulation order.

At the receiver, a matched filter (correlator) is used to detect the received signal, $r(t)$, as follows:

$$
z_{i}(t)=\int_{0}^{T} r(t) s_{i}(t) d t \quad i=1, \cdots, M
$$

Where $s_{i}(t)$ is the candidate prototype signal that is known a priori to the receiver. The receiver chooses the signal $s_{i}(t)$ as the detected signal that matches best or has the largest correlation with the received signal $r(t)$. Figure 2.8 is the diagram of the receiver and here $\hat{s}_{i}(t)$ is the signal that the receiver detects.

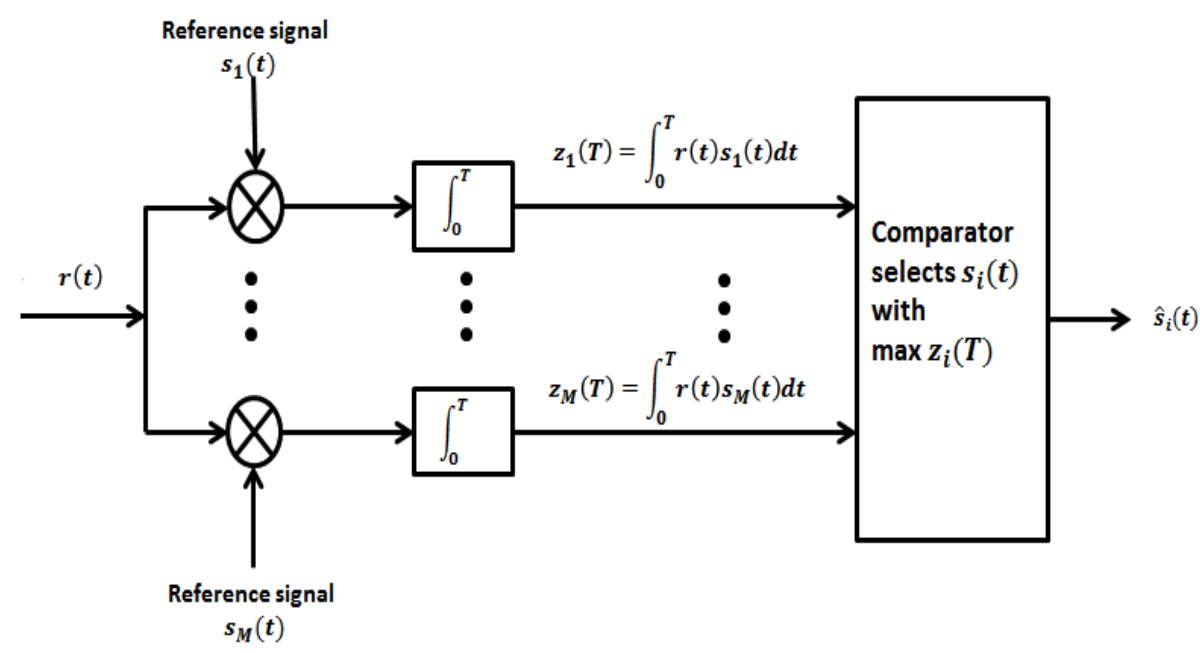

Figure 2.8: Block diagram of a correlator receiver in ASK modulation. 


\subsubsection{On-Off Keying (OOK)}

One method that is used to modulate $\mathrm{THz}$ pulses is called on-off keying (OOK) that is a binary ASK modulation. In this method, by changing the amplitude of pulses we can create two different states to represent a "0" or a " 1 ". That is, a pulse with amplitude $A$ corresponds to a " 1 " and a pulse with amplitude zero (no pulse is sent) corresponds to a "0". Figure 2.9 is an example of OOK modulation.

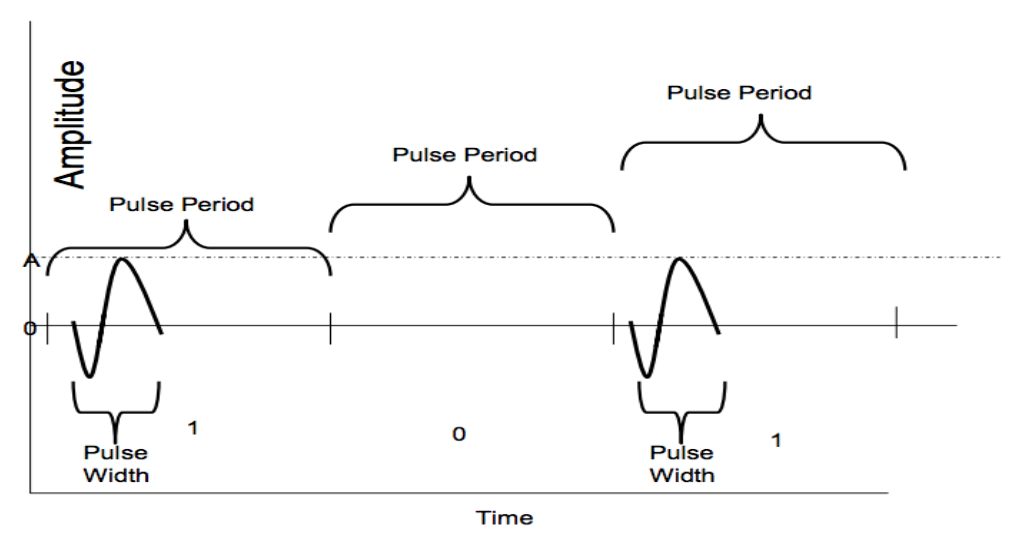

Figure 2.9: An example of OOK modulation [9].

The block diagram of the ASK receiver in Figure 2.8 is simplified to a binary detector. Here, the correlation receiver is configured as a signal matched filter with the reference signal that is the difference between the binary prototype signals, $s_{1}(t)-s_{2}(t)$. Figure 2.10 shows the block diagram of the receiver. Here $\gamma$ is the amplitude threshold that is $\frac{A+0}{2}=\frac{A}{2}$. 


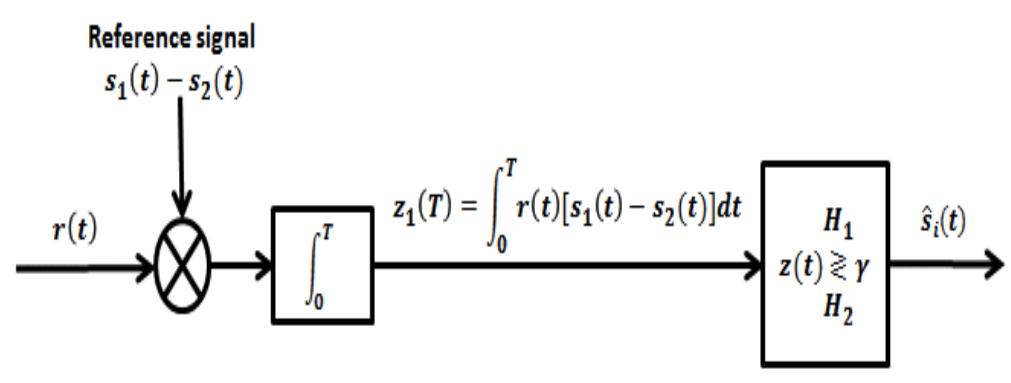

Figure 2.10: The block diagram of OOK receiver.

\subsubsection{Frequency-Domain Differential PSK (FD-DPSK)}

Rhee et al. [49] proposed a method to phase modulate pulses, which is called frequency domain differential phase shift keying (FD-DPSK). In this method, each pulse corresponds to several number of bits. Here, the frequency spectrum of each pulse is phase modulated. That is, the frequency spectrum of each pulse is divided into several bands. Each band corresponds to one symbol. The first band is always modulated with phase 0 and the other bands are phase modulated with respect to their previous band. As a result, having $n+1$ bands, we can modulate $n$ symbols in this method.

Rhee et al. used an acousto-optic modulator (AOM) with spectral resolution $n+1$ to modulate pulses. AOM has a piezoelectric transducer that is attached to a glass. An oscillating electric signal drives the transducer to vibrate and as a result sound waves are created in the glass. Figure 2.11 shows the transmitter's structure in this system. 


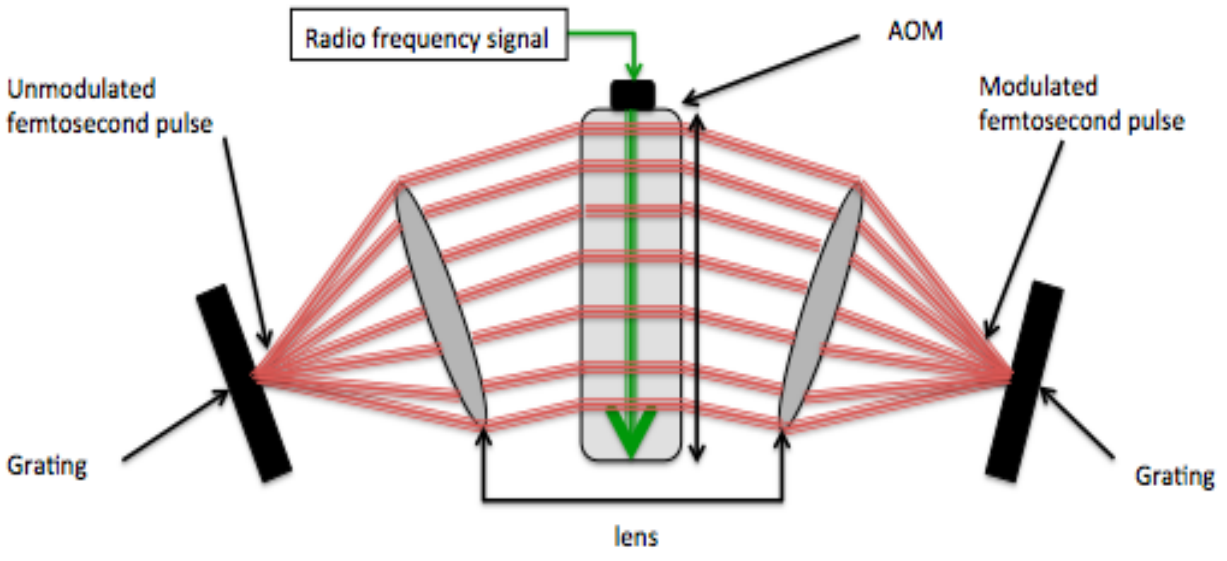

Figure 2.11: Transmitter structure using an AOM.

Here, a transmitter consists of a femtosecond pulse laser and a sequence of grating $\rightarrow$ lens $\rightarrow$ AOM $\rightarrow$ lens $\rightarrow$ grating. The first grating and lens are placed before the AOM and are used to slice the pulse into separate frequency bands. A radio frequency $(\mathrm{RF})$ signal containing the data sequence goes into the AOM. This RF signal creates a traveling acoustic wave that propagates through the AOM and is used to modulate the sliced pulsed signal. The modulated pulse is then passed through another grating and lens. As a consequence, the output pulse is a superposition of all modulated bands.

In the case of DBPSK, where each symbol carries one bit of data, $n$ bits of data from $a_{i}=\{0,1\}$ are considered. In this case, for each of the $n+1$ spectral bands we have:

$$
b_{i}= \begin{cases}0 & \text { for } i=0, \\ \left(a_{i}+b_{i-1}\right) \bmod 2 & \text { for } i=1, \cdots, n .\end{cases}
$$

Then, the phase for each band is found from the equation below:

$$
\phi_{i}=\pi b_{i}
$$

The assumption here is that the portion of the pulsed signal that is used in 
modulation is a rectangle function with a constant amplitude and a spectral bandwidth of $\Delta f$. The following relation shows the definition of a rectangular function with a constant amplitude 1 :

$$
\operatorname{rect}(x, a)= \begin{cases}1 & \text { for }-|a| / 2 \leq x \leq|a| / 2 \\ 0 & \text { otherwise }\end{cases}
$$

The spectrum of the pulse is divided into $n+1$ spectral bands and each band is modulated from (2.38) as below:

$$
E(f)=\sum_{i=0}^{n} \mathrm{e}^{j \phi_{i}} S\left(f-f_{i}, \delta f\right)
$$

Here, $E(f)$ is the spectral complex value of the signal as a function of frequency $f, i$ corresponds to each spectral band, and $S\left(f-f_{i}, \delta f\right)$ is the spectral value of $i$ th band. Also, it should be noted that $f_{i}=f_{0}+i \delta f$ is the central frequency, and $\delta f=\Delta f /(n+1)$ is the bandwidth of the $i$ th band. Where, $f_{0}$ is the central frequency of the first spectral band and $\Delta f$ is the bandwidth of the portion of the signal's spectrum that is used for communication.

The signal is then passed through the channel and is changed based on the channel model. At the receiver a Mach-Zehnder or Sagnac interferometer is used with a device for wavelength shift. The received signal, $R(f)$, is first sliced into $n+1$ bands. Then, a copy of the signal is shifted in frequency by $\Delta f$. As a consequence, the shifted $(i-1)$ th band has the central frequency of the unshifted $i$ th band. Then the $i$ th element at the receiver is found from the following equations:

$$
E_{\text {det }}^{i}(f)= \begin{cases}\frac{1}{2} \operatorname{rect}\left(f-f_{0}, \delta f\right) \mathrm{e}^{j \phi_{0}} & \text { for } i=0 \\ \frac{1}{2} \operatorname{rect}\left(f-f_{i}, \delta f\right)\left[\mathrm{e}^{j \phi_{i-1}}+\mathrm{e}^{j \phi_{i}}\right] & \text { for } i=1, \cdots, n \\ \frac{1}{2} \operatorname{rect}\left(f-f_{n+1}, \delta f\right) \mathrm{e}^{j \phi_{n}} & \text { for } i=n+1\end{cases}
$$


From $(2.37) a_{i}=\left(b_{i}-b_{i-1}\right)$. As a result we can find the power spectrum for $i=1, \cdots, n$ as below:

$$
I_{\text {det }}^{i}(f)=\left|\tilde{E}_{\text {det }}^{i}(f)\right|^{2}=\frac{1}{2} \operatorname{rect}\left(f-f_{i}, \delta f\right)\left[1+\cos \left(\pi a_{i}\right)\right]
$$

From (2.42), the phase information is converted to amplitude modulation. that is a 0 bit $\left(a_{i}=0\right)$ produces the intensity of 1 and a 1 bit $\left(a_{i}=1\right)$ produces the intensity of 0 . As a result, the corresponding bit to each spectral band at the receiver can be found based on what we saw in OOK modulation in subsection 2.6.2. 
Chapter 3

\section{CHANNEL CHARACTERIZATION}

In this chapter we characterize indoor $\mathrm{THz}$ channels and develop models that can be used in simulation and analysis of terahertz $(\mathrm{THz})$ communication systems. We first describe our testbed and then explain the channel measurements we conducted to develop our channel model. The data clearly shows significant attenuation due to absorption of some frequencies in the air, In section 3.3.1 we develop a model for this attenuation. Our measurements were conducted using collimating lenses at both the transmitter and receiver as well as without. A detailed description of the directional channel produced using collimating lenses is in section 3.3 and of the non-directional channel is in section 3.4.

\section{TESTBED AND EXPERIMENTAL DESIGN}

We use the commercially available Picometrix T-Ray 4000 system that is provided by Advanced Photonix, Inc (API) [2] in our measurements. In this system, the transmitter generates pulses that travel through the air and are then received by a synchronized receiver to be processed. The time resolution of the detector is 78 femtoseconds, the signal to noise ratio (SNR) is equal to $80 \mathrm{~dB}$ at $0.5 \mathrm{THz}$ frequency, and each generated pulse covers the frequency range of 0-2 THz. The transmitter sends pulses and receiver captures and saves the average of 10000 pulses for our measurements. In this system, both transmitter and receiver have photoconductive antenna (PCA) and can have collimating or focus lenses in front 
of them to fully collimate or focus the signal, respectively.

A collimating lens is a highly directional lens which produces parallel beams with the focus at infinity. We call the channel that has a collimating lens a $d i$ rectional channel. Removing collimating lenses at transmitter and receiver results in a different channel, which we call non-directional channel. We experiment with these two very different channels to analyze the need for directional transmission. In the first set of experiments, we put a collimating lens at both the transmitter and receiver. We set the initial transmitter-receiver distance to $11.5 \mathrm{~cm}$ and we go up to the transmitter-receiver distance of $66.5 \mathrm{~cm}$ with increments of $5 \mathrm{~cm}$. In the next set of experiments we remove the collimating lens from both the transmitter and the receiver. Also, to better analyze the antenna in the non-directional channel we change transmitter-receiver angle up to 40 degrees.

As the $\mathrm{THz}$ signal propagates through the channel, it is attenuated due to the resonance of some molecules in the atmosphere at specific frequencies. These resonance modes are due to rotational or vibrational transitions, that correspond to the $\mathrm{THz}$ frequency range. Many rotational and vibrational frequencies of water vapor lie in the terahertz domain. To explore the effect of water vapor, we conduct measurements in dry air and in humid air.

The humid air experiment has a relative humidity $(\mathrm{RH})$ of $42 \%$. For the dry case, we constructed a plastic tunnel around the transmitter and the receiver and blow dry air into the tunnel continuously to make sure that the $\mathrm{RH}$ remains at $0 \%$. It should be noted that the temperature for all these measurements is set to $70^{\circ} \mathrm{F}$. Figure 3.1 shows the lab setup for creating the dry channel. 


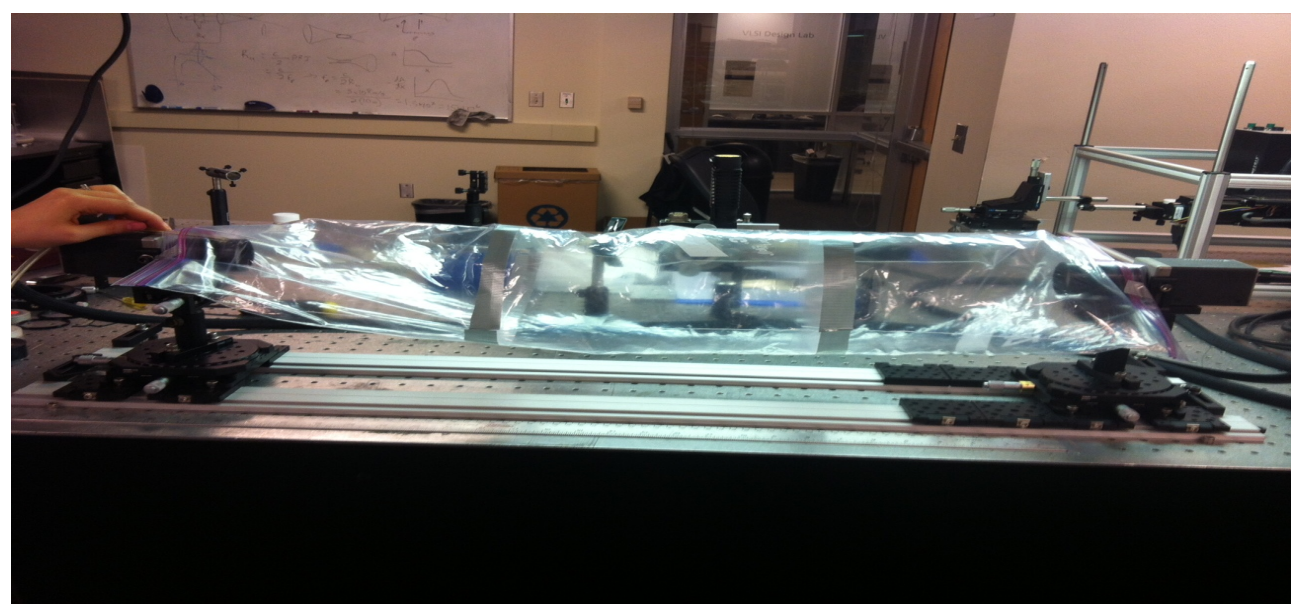

Figure 3.1: The lab setup for creating dry channel.

Table 3.1 is the outline of our experimental design. For the collimating lens case we varied the transmitter-receiver distance from $11.5 \mathrm{~cm}$ to $66.5 \mathrm{~cm}$ in 5 $\mathrm{cm}$ increments. We conducted measurements at $0 \%$ and $42 \% \mathrm{RH}$. For the nondirectional case, we kept the humidity at $42 \%$ but varied the transmitter-receiver (Tx-Rx) distance from $20 \mathrm{~cm}$ to $70 \mathrm{~cm}$ in $5 \mathrm{~cm}$ increments.

Table 3.1: Summary of variable parameters.

\begin{tabular}{|l|l|l|}
\hline Lens Type & Collimating & No-lens \\
\hline Tx-Rx Distance & $11.5-66.5 \mathrm{~cm}$, increments of $5 \mathrm{~cm}$ & $20-70 \mathrm{~cm}$, increments of $5 \mathrm{~cm}$ \\
\hline Humidity & $0 \%, 42 \%$ & $42 \%$ \\
\hline Tx-Rx Angle & $0^{\circ}$ & $0^{\circ}-39.4^{\circ}$, increments of $<5^{\circ}$ \\
\hline
\end{tabular}

Note that, in the no-lens case, the receiver angle to the pivot point is changing from $0^{\circ}$ to $40^{\circ}$ in $5^{\circ}$ increments. However, as we will discuss in section 3.4.1, the actual transmitter-receiver angle is changed between $0^{\circ}-39.4^{\circ}$ with increments of $2.92^{\circ}-4.98^{\circ}$ 


\section{OVERVIEW OF THE RECEIVED SIGNALS}

As mentioned earlier, for each measurement the receiver captures an average of 10000 time domain pulses. Each captured signal (pulse) has 4096 time samples where the sample time is $T_{s}=78.125 \times 10^{-15} \mathrm{~s}$. Figures 3.2 and 3.3 show pulses that the receiver receives for a directional channel for transmitter-receiver distance of $21.5 \mathrm{~cm}$ when the $\mathrm{RH}$ is $42 \%$ and $0 \%$, respectively.

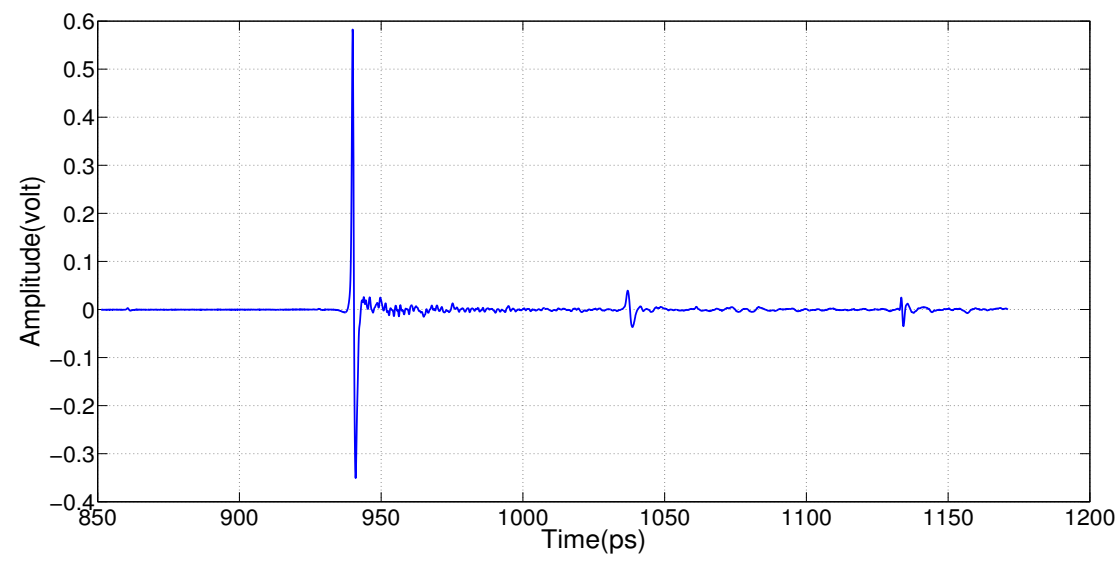

Figure 3.2: Received pulse in directional channel for transmitter-receiver distance of 21.5 $\mathrm{cm}$ and $\mathrm{RH}$ of $42 \%$. 


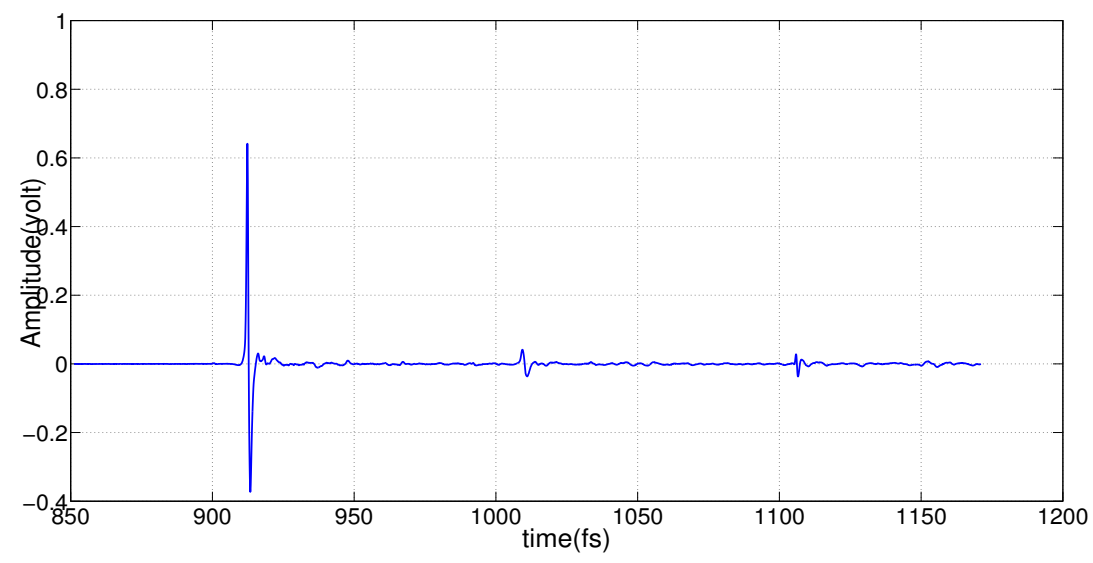

Figure 3.3: Received pulse in directional channel for transmitter-receiver distance of 21.5 $\mathrm{cm}$ and $\mathrm{RH}$ of $0 \%$.

Figure 3.4 is the time domain signal for the non-directional channel when the transmitter-receiver distance is $20 \mathrm{~cm}$.

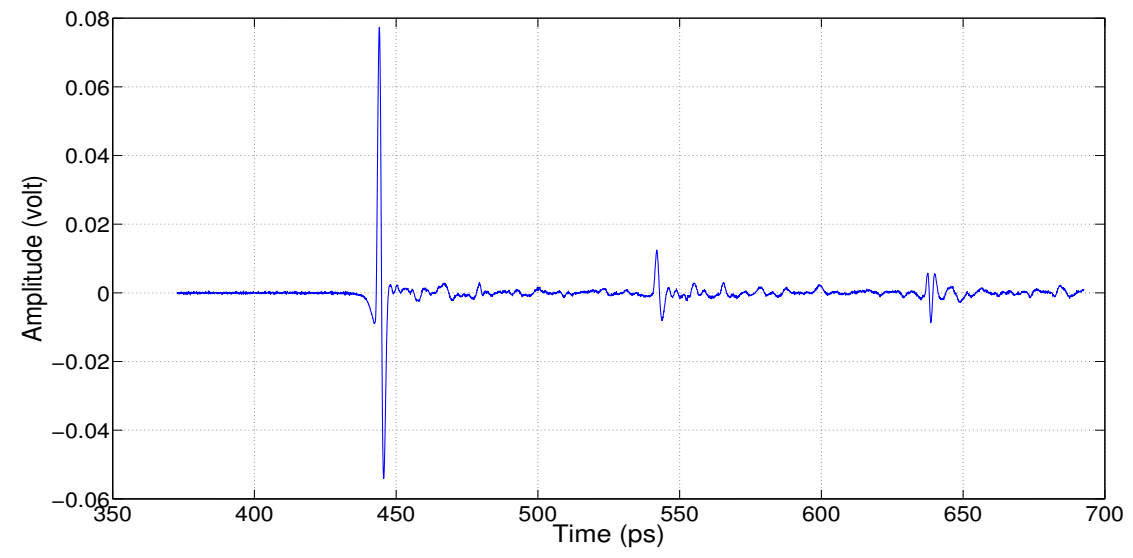

Figure 3.4: Received pulse in non-directional channel for transmitter-receiver distance of $20 \mathrm{~cm}$.

For longer transmitter-receiver distances, the receiver receives the signal later than for the shorter transmitter-receiver distances. Therefore, x-axis shows a longer signal travel time. As all three figures show, there are oscillations to the right of the 
main pulse. These oscillations are due to back-reflections off the antenna substrate of the Picometrix system [69]. To decrease the effect of these oscillations, we first filter the pulse with a 500 index window. Hence, the length of the window is $500 \times T_{s}$, where $T_{s}$ is the sample time and is equal to $78.125 \times 10^{-15} \mathrm{~s}$. The corresponding Matlab code is in Appendix A. Figures 3.5, 3.6, and 3.7 show the filtered pulse from Figures 3.2, 3.3, and 3.4, respectively.

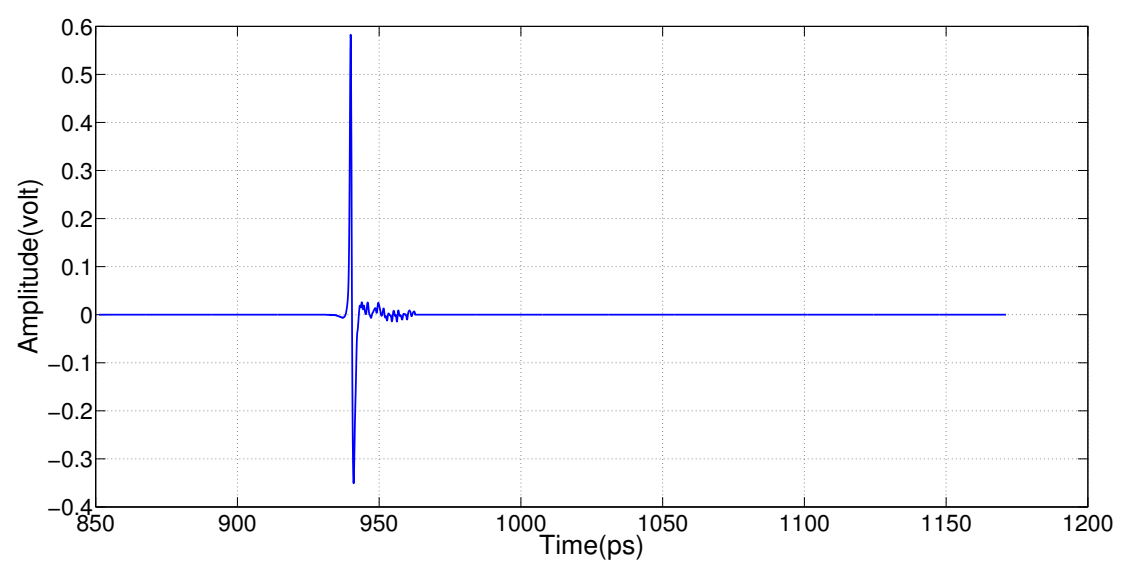

Figure 3.5: Filtered received pulse for directional channel when transmitter-receiver distance is $21.5 \mathrm{~cm}$ and $\mathrm{RH}$ is equal to $42 \%$. 


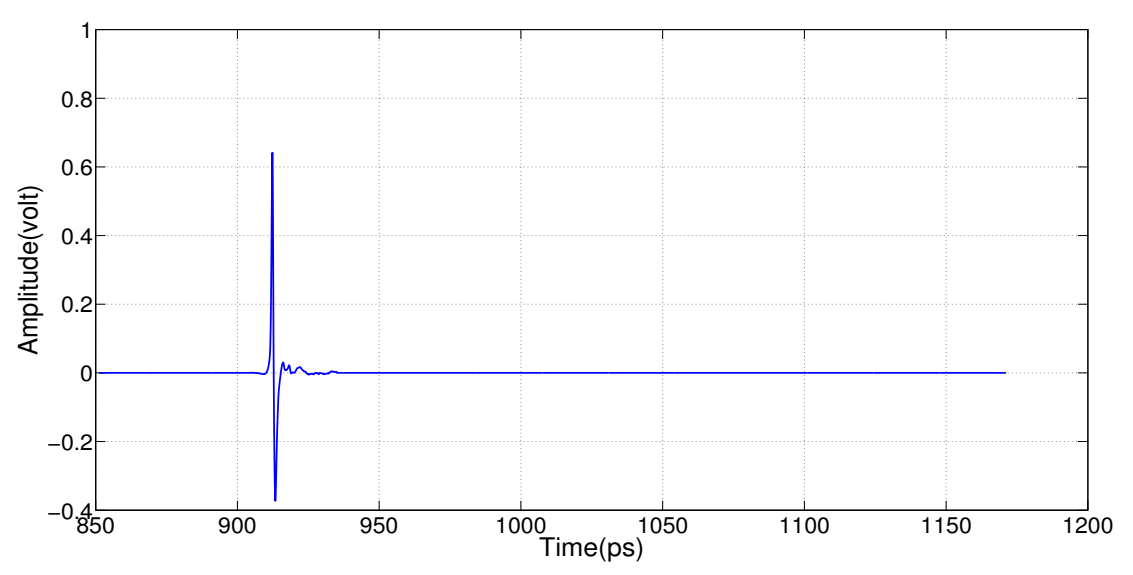

Figure 3.6: Filtered received pulse for directional channel when transmitter-receiver distance is $21.5 \mathrm{~cm}$ and $\mathrm{RH}$ is equal to $0 \%$.

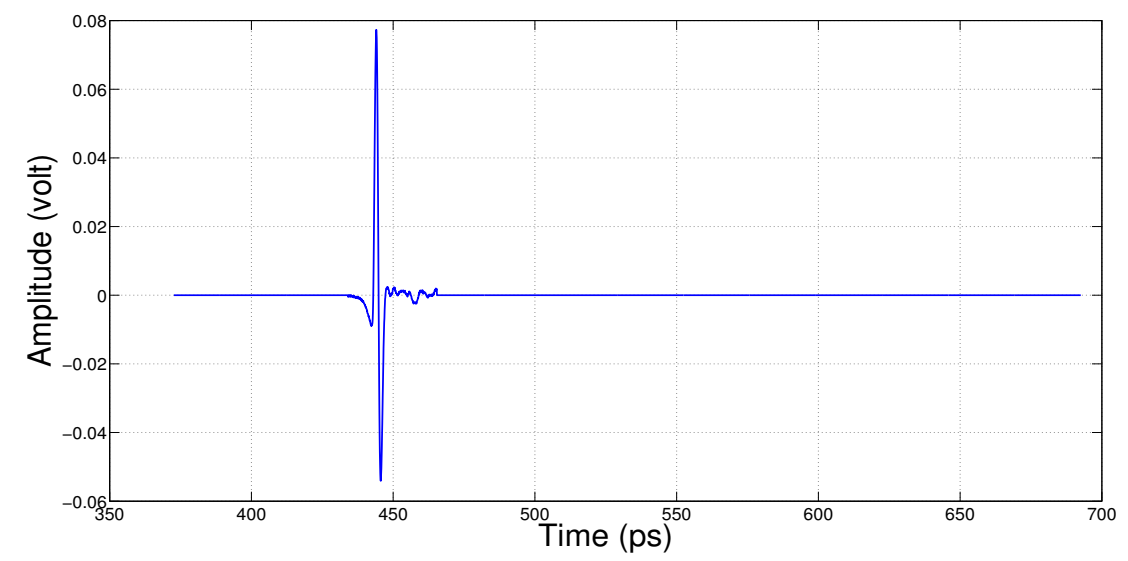

Figure 3.7: Filtered received pulse for non-directional channel when transmitter-receiver distance is $20 \mathrm{~cm}$.

\section{DIRECTIONAL CHANNEL CHARACTERIZATION}

As we discussed earlier, in the directional channel both the transmitter and receiver have collimating lenses and the receiver is aligned with the transmitter to receive the transmitted signal. In other words the transmitter-receiver angle is set to 0 
degrees. Figure 3.8 shows the total power vs. transmitter-receiver distance for $0 \%$ and $42 \%$ RH levels.

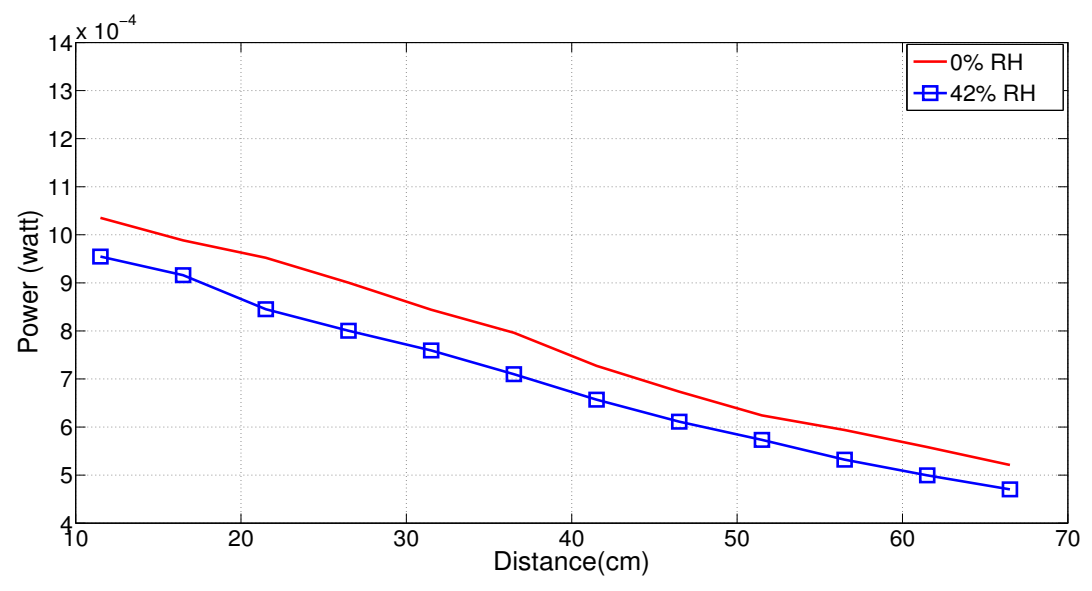

Figure 3.8: Power vs. transmitter-receiver distance of the received signal in directional channel for $0 \%$ and $42 \%$ relative humidities.

As we see, due to attenuation caused by water vapor molecules, the received power in the case of $42 \% \mathrm{RH}$ is less than the case of $0 \% \mathrm{RH}$. Figure 3.9 shows the result of comparing the frequency spectrum of the signals with $0 \%$ and $42 \% \mathrm{RH}$ from Figures 3.5 and 3.6. 


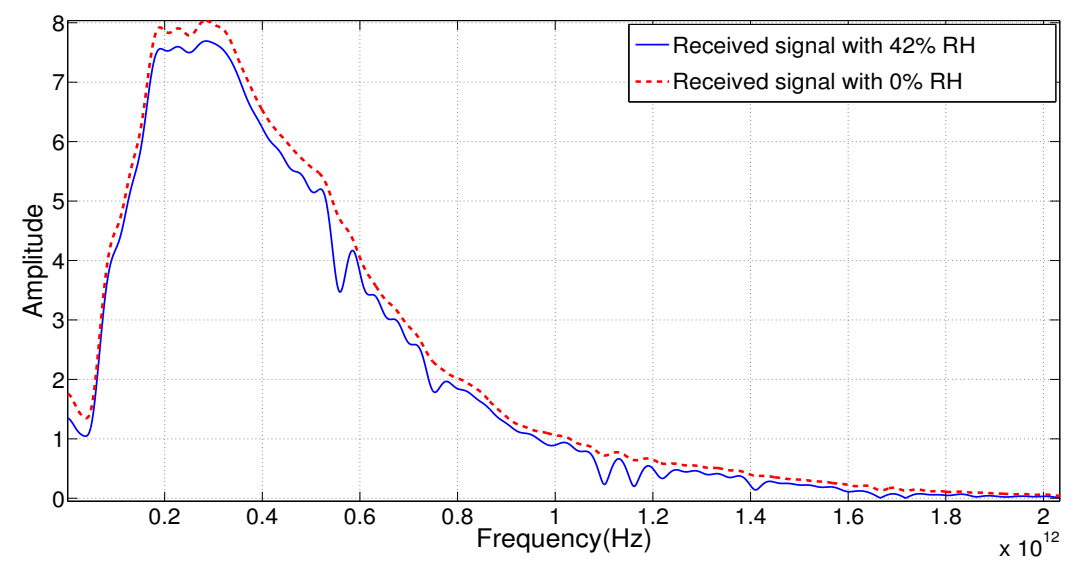

Figure 3.9: Comparison of the frequency spectrum of the signals with $42 \%$ and $0 \%$ relative humidity $(\mathrm{RH})$ with transmitter-receiver distance of $21.5 \mathrm{~cm}$. The solid line corresponds to signal with $42 \% \mathrm{RH}$ and the dotted line corresponds to the signal with $0 \% \mathrm{RH}$.

The figure shows that the detected $\mathrm{THz}$ energy is mainly below $2 \mathrm{THz}$ in our system. Beyond $2 \mathrm{THz}$ the signal is too weak to be distinguished from noise. Also, we see that in the received signal with $42 \% \mathrm{RH}$ some frequencies are severely attenuated. These troughs correspond to the atmospheric attenuation that happens due to the presence of water vapor molecules in the air.

Now lets investigate the received power for some individual bands. First, from Figure 3.9 we select three frequencies that are not absorbed by water vapor molecules. Then, we consider a $1 \mathrm{GHz}$ band centered at these frequencies and calculate their received power. Figures 3.10, 3.11, and 3.12 show the received power for $0 \%$ and $42 \%$ humidities for frequency bands centered at $110 \mathrm{GHz}, 170$ $\mathrm{GHz}$, and $400 \mathrm{GHz}$, respectively. 


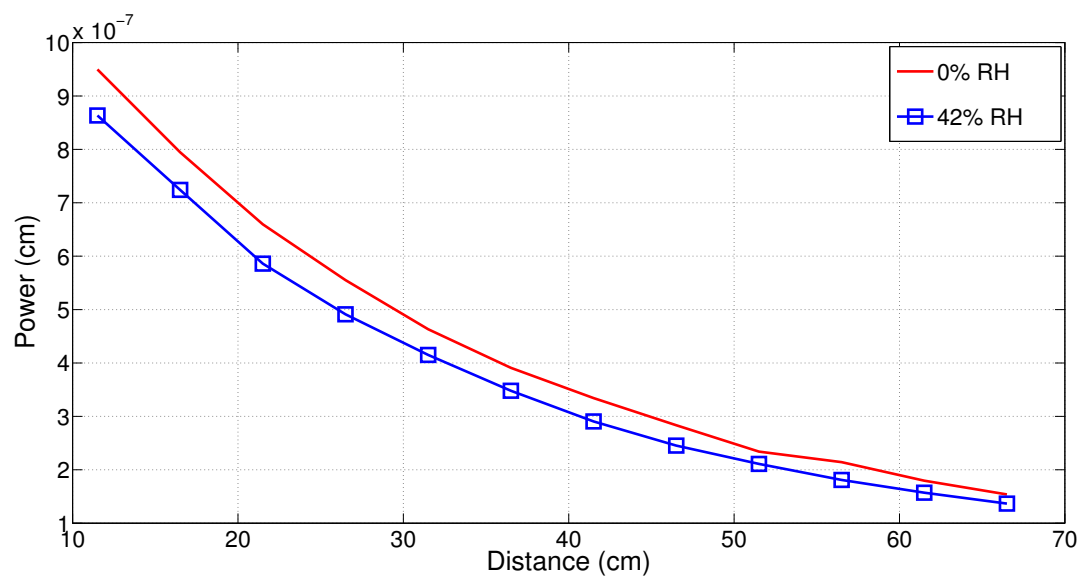

Figure 3.10: Received power of $110 \mathrm{GHz}$ band with $1 \mathrm{GHz}$ bandwidth for $0 \%$ and $42 \%$ RH levels.

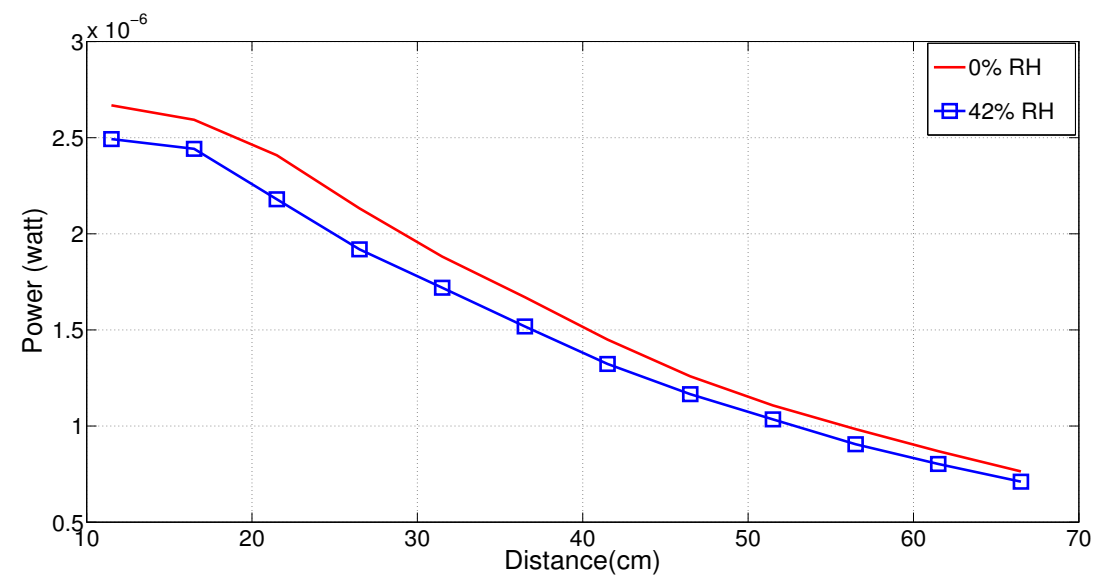

Figure 3.11: Received power of $170 \mathrm{GHz}$ band with $1 \mathrm{GHz}$ bandwidth for $0 \%$ and $42 \%$ RH levels. 


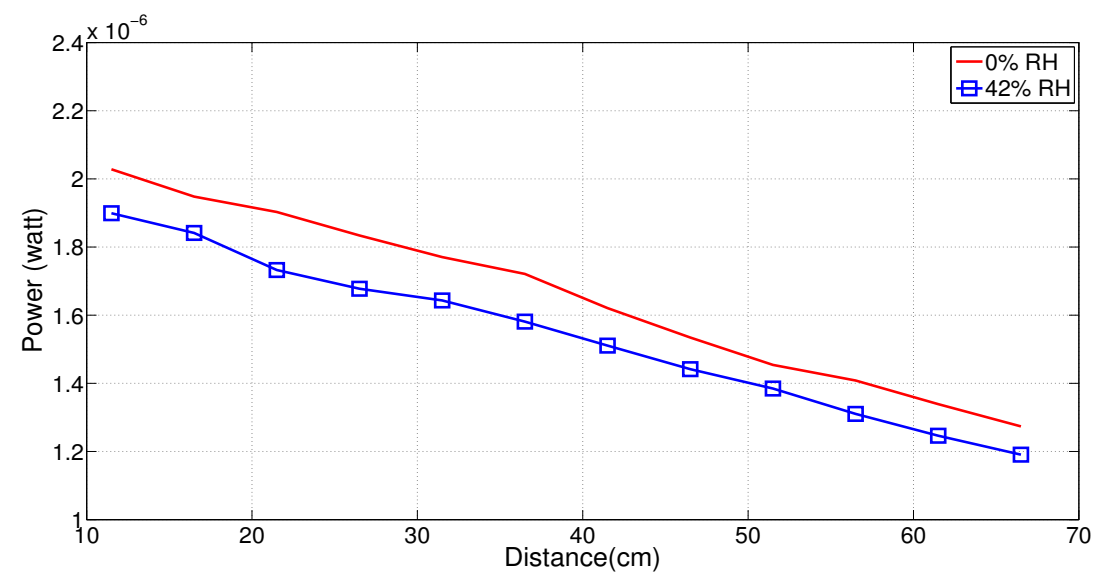

Figure 3.12: Received power of $400 \mathrm{GHz}$ band with $1 \mathrm{GHz}$ bandwidth for $0 \%$ and $42 \%$ RH levels.

The result shows that the received power corresponding to $42 \% \mathrm{RH}$ is less than received power corresponding to $0 \% \mathrm{RH}$ in all three figures. For example, in Figure 3.12 the received power of $42 \% \mathrm{RH}$ is $\frac{1.899 \times 10^{-6}}{2.028 \times 10^{-6}}=0.9364$ that of the $0 \% \mathrm{RH}$ case. The reason is that water vapor molecules absorb the energy of the signal as it propagates through the air. Therefore, with higher humidity, the signal power is attenuated more.

Now consider three frequency bands of $560 \mathrm{GHz}, 750 \mathrm{GHz}$, and $1.1 \mathrm{THz}$ where there is a higher water vapor attenuation. Figures 3.13, 3.14, and 3.15 show the received power corresponding to these three bands, respectively. 


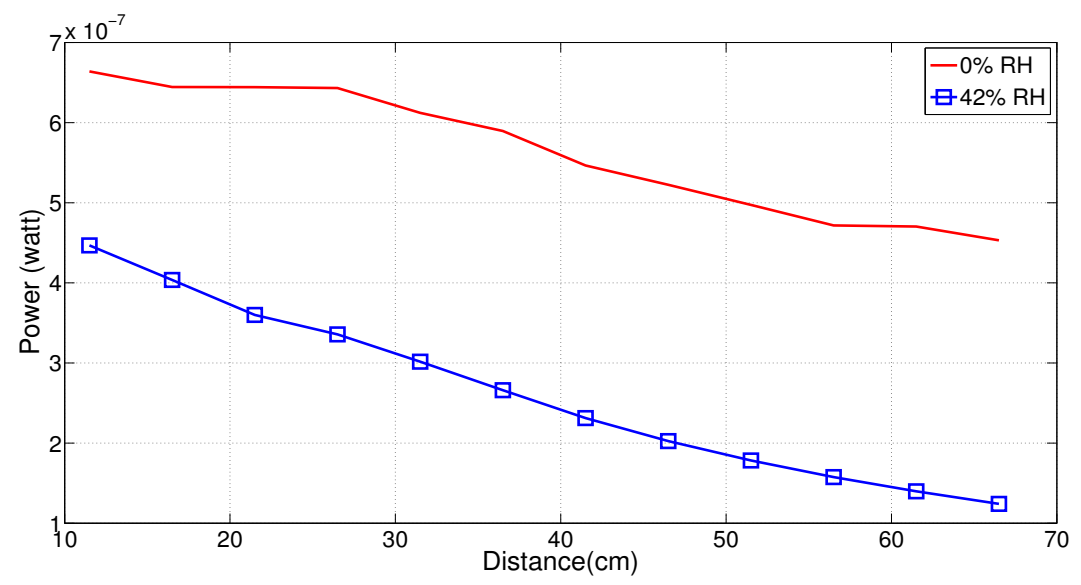

Figure 3.13: Received power of $560 \mathrm{GHz}$ band with $1 \mathrm{GHz}$ bandwidth for $0 \%$ and $42 \%$ RH levels.

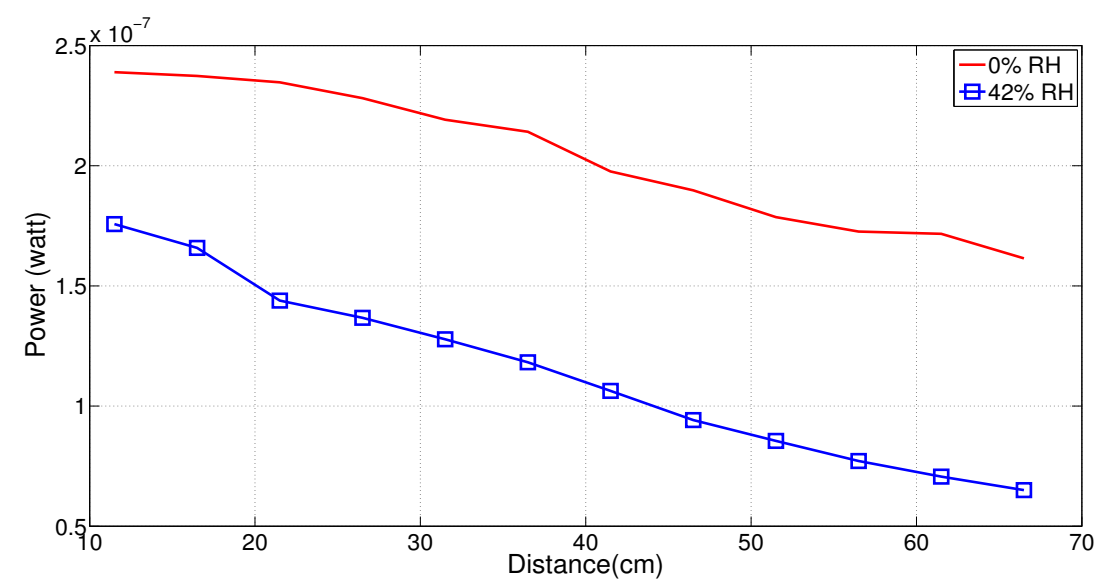

Figure 3.14: Received power of $750 \mathrm{GHz}$ band with $1 \mathrm{GHz}$ bandwidth for $0 \%$ and $42 \%$ RH levels. 


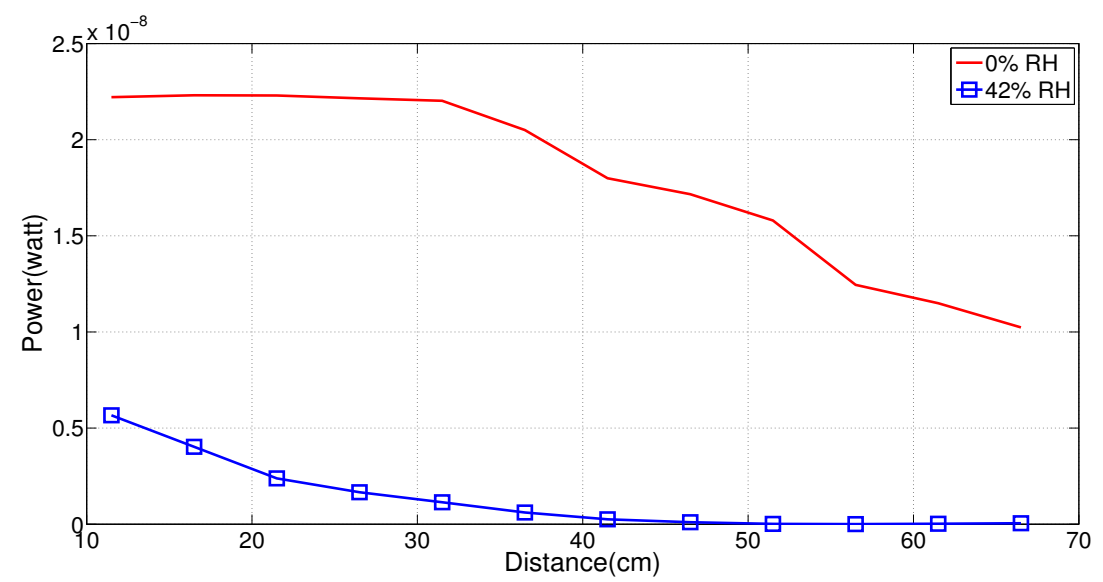

Figure 3.15: Received power of $1.1 \mathrm{THz}(1100 \mathrm{GHz})$ band with $1 \mathrm{GHz}$ bandwidth for $0 \%$ and $42 \%$ RH levels.

The results show a much greater difference between the received power corresponding to $0 \%$ and $42 \% \mathrm{RH}$ ranging from $\frac{1.757 \times 10^{-7}}{2.389 \times 10^{-7}}=0.7355$ to $\frac{6.445 \times 10^{-12}}{1.245 \times 10^{-8}}=$ $5.1767 \times 10^{-4}$. Comparing the result of these three figures with the result of 80 $\mathrm{GHz}, 170 \mathrm{GHz}$, and $400 \mathrm{GHz}$ bands shows the significant effect of water vapor attenuation in these bands. In section 3.3.1 we develop a model for absorption due to water vapor.

Another important observation we can make from figures 3.10 to 3.12 is that collimating lenses are not behaving exactly as explained. Since we use collimating lens, we expect parallel beams with minimum of divergence. In other words, the power for $0 \% \mathrm{RH}$ should not change with distance. However, the result of above figures show a decrease of received power for the $0 \% \mathrm{RH}$ case with distance. A possible explanation is that the beam diverges causing the decrease in the received power. In the section 3.3.2 we will model the diverging angle $\theta$. 


\subsubsection{Modeling Attenuation Due to Absorption}

When the frequency of an electromagnetic signal reaches the resonant frequency of some molecule in the atmosphere, the molecule absorbs the signal's energy and changes its state between two quantum mechanical states. The absorption that occurs due to a transition between the two states is called an absorption line. The $\mathrm{THz}$ frequency spectrum is composed of many absorption lines that are unique for each gas molecule. The energy associated with the quantum mechanical change primarily determines the frequency of the absorption line but the frequency can be shifted by some factors like changes in temperature and pressure of the atmosphere. For our channel model to be used in simulations, we need an accurate formula to compute this atmospheric frequency-specific attenuation. Therefore, we need a precise measurement of the atmospheric attenuation corresponding to each frequency component.

At each of the absorption lines, molecules absorb energy, i.e., photons, from the radiated field at different frequencies or wavenumbers, where wavenumber is the number of waves per centimeter.

$$
\nu\left(\mathrm{cm}^{-1}\right)=\frac{f(\mathrm{~Hz})}{c(\mathrm{~cm} / \mathrm{s})}
$$

Here, $f$ is the frequency in $\mathrm{Hz}$, and $c$ is the speed of light, $2.997925 \times 10^{10} \mathrm{~cm} / \mathrm{s}$.

Molecular absorption lines always have a width and shape. It is common for a line to be characterized by its intensity and width instead of its shape. This produces a unique spectrum for each gas molecule. The shape, Gaussian or Lorentzian distribution, is determined by the broadening mechanism while the intensity of a line is obtained by integrating the area under the absorption line and is proportional to the temperature and amount of the absorbing substance present in the environment. 
Given a set of parameters, absorption lines can be modeled at any temperature and pressure. The collection of these parameters are called line lists and can be obtained from high-resolution transmission molecular absorption (HITRAN) database [53]. Table 3.2 shows the list of parameters that we use in our model for modeling the absorption lines.

Table 3.2: HITRAN line by line parameters

\begin{tabular}{ccc}
\hline \hline Name & Symbol & Unit \\
\hline Line center at $P_{0}$ and $T_{0}$ & $\nu_{c}^{0}$ & $\mathrm{~cm}^{-1}$ \\
Air broadened halfwidth at $T_{0}$ & $\alpha_{f}^{0}$ & $\mathrm{~cm}^{-1}$ \\
Self broadened halfwidth at $T_{0}$ & $\alpha_{s}^{0}$ & $\mathrm{~cm}^{-1}$ \\
Line intensity at $T_{0}$ & $S_{0}$ & $\mathrm{~cm}^{-1} /\left(\right.$ molecule $\left.\times \mathrm{cm}^{-2}\right)$ \\
\hline
\end{tabular}

Where, $T_{0}$ is the reference value for temperature and is equal to $294 \mathrm{~K}\left(\sim 70^{\circ}\right.$ $\mathrm{F}$ or $\left.\sim 21^{\circ} \mathrm{C}\right) . P_{0}$ is the reference value for atmospheric pressure and is equal to 1 atmosphere.

To simulate the absorption spectrum in a given frequency range, we first calculate the absorption lines of each gas molecule. The effect of Lorentz line broadening due to collisions between gas molecules is characterized by a half-width parameter that determines the width and shape of the lines. $\alpha$ is the Lorentz half-width at half of the max value. Half-width is obtained from mean time between collisions and is proportional to the number of collisions per unit time and changes with the pressure and temperature. $\alpha_{a}^{0}$ is the Lorentz half-width at half max between collisions of the gas molecule with other molecules in air and $\alpha_{s}^{0}$ is the half-width at half max for collisions between gas molecules of the same type. The Lorentz half-width $\alpha$ is computed as the weighted sum of air-broadened and self-broadened 
half-widths as in (3.2).

$$
\alpha=\left[(1-q) \alpha_{a}^{0}+q \alpha_{s}^{0}\right]
$$

Where, $q$ is the mixing ratio of the absorber molecule in the air and is dimensionless.

We use the Van Vleck-Weisskopf (VVW) line shape to model the shape of the absorption lines. VVW line shape equation is as below [66]:

$$
g(\nu)(\mathrm{cm})=\frac{1}{\pi}\left(\frac{\nu}{\nu_{0}}\right)^{2}\left[\frac{\alpha}{\left(\nu-\nu_{0}\right)^{2}+\alpha^{2}}+\frac{\alpha}{\left(\nu+\nu_{0}\right)^{2}+\alpha^{2}}\right]
$$

Here, $\nu_{0}$ is the wavenumber $\left(\mathrm{cm}^{-1}\right)$ of the line center and $\nu$ is the wavenumber $\left(\mathrm{cm}^{-1}\right)$ of the frequency for which we want to calculate the absorption.

Based on the Beer-Lambert law, the molecular absorption spectrum is quantitatively related to the presented amount of material. (3.4) shows how to calculate the absorption coefficient $\kappa(\nu)$.

$$
\kappa(\nu)\left(\mathrm{cm}^{-1}\right)=u S g(\nu)
$$

$S$ is the line strength in $\mathrm{cm}^{-1} /\left(\right.$ molecule $\left.\times \mathrm{cm}^{-2}\right) . u$ is the absorber thickness in molecule $/ \mathrm{cm}^{3}$ that shows the number of absorber molecules in one $\mathrm{cm}^{3}$ of environment.

To calculate the total absorption coefficient of a specific molecule at any wavenumber, we calculate the summation of the attenuation due to each absorption line as below.

$$
K(\nu)=\sum_{i} \kappa_{i}(\nu)
$$

In our experiment, we consider absorption lines up to $2 \mathrm{THz}$ (wavenumber $=$ $\left.67 \mathrm{~cm}^{-1}\right)$ for water vapor $\left(\mathrm{H}_{2} \mathrm{O}\right)$, oxygen $\left(\mathrm{O}_{2}\right)$, nitrogen $\left(\mathrm{N}_{2}\right)$, and carbon dioxide $\left(\mathrm{CO}_{2}\right)$. Since the pressure and temperature of our experimental environment are the same as the reference values $T_{0}$ and $P_{0}$, there is no need to adjust the value of the parameters. 


\section{Calculating Water Vapor Absorption Coefficient in our channel}

To calculate absorption coefficient of water vapor, we need to first calculate water vapor thickness $u$ and also its mixing ratio $q$ in our channel.

The pressure of our channel, $P$, is equal to the reference pressure $P_{0}$, that is 1 atm or 101325 pa. We also need to find water vapor pressure $P_{v}$. For that we find the water vapor saturation pressure $P_{v s}$ from the following equation.

$$
P_{v s}=6.1078 \times 10^{\frac{7.5 \times T}{T+237.3}}
$$

Where $T$ is the temperature in Celsius and is $21^{\circ} \mathrm{C}$ for the day on which the data is collected. From the equation, $P_{v s}$ becomes 24.8680 millibar. Note that 1 millibar $=0.000986923267 \mathrm{~atm}=100 \mathrm{pa}$.

Based on the result from (3.6) and having $42 \%$ relative humidity $\Phi$, water vapor pressure $P_{v}$ is equal to 10.4446 millibar.

$$
P_{v}=P_{v s} \times \Phi
$$

To find the corresponding thickness of water vapor, $u$, we first calculate the water vapor density in $1 \mathrm{~cm}^{3}$ of our channel from the following equation.

$$
\rho\left(\mathrm{kg} / \mathrm{m}^{3}\right)=\frac{P_{v} M_{v}}{R T}
$$

Here, $\rho$ is the density of the water vapor in $\mathrm{kg} / \mathrm{m}^{3}, P_{v}$ is partial pressure of water vapor in pa, $M_{v}$ is the molar mass of water vapor and is equal to $0.018016(\mathrm{~kg} /-$ mole), and $R$ is the universal gas constant that is equal to $8.314 \mathrm{~J} /(\mathrm{Kmole}) . T$ is the temperature in ${ }^{\circ} \mathrm{K}$. (Note that, $1 \mathrm{~J}=\mathrm{Nm}$ and $1 \mathrm{pa}=\mathrm{N} / \mathrm{m}^{2}$ ). The result is then multiplied by 0.001 to give the water vapor density in $\mathrm{gr} / \mathrm{cm}^{3}$.

Since each gram of water vapor is 0.0555084350618 moles and each mole has $6.02214129 \times 10^{23}$ water molecules, multiplying the value of water vapor density 
from (3.8) by these two values gives us the number of water vapor molecules in one $\mathrm{cm}^{3}$. Therefore, $u$ is equal to $2.556 \times 10^{17}\left(\right.$ molecule $\left./ \mathrm{cm}^{3}\right)$. To find the water vapor mixing ratio $q$, we use the following equation.

$$
q=\frac{0.622 \times P_{v}}{P-P_{v}}
$$

Having found the required values, we use HITRAN database with the use of JavaHAWKS software package [52] and equations (3.2), (3.3), and (3.4) to calculate water vapor absorption coefficient for frequencies up to $2 \mathrm{THz}\left(67 \mathrm{~cm}^{-1}\right)$. Figure 3.16 shows the absorption coefficient of water vapor in $\mathrm{dB} / \mathrm{cm}$ when the $\mathrm{RH}$ is $42 \%$.

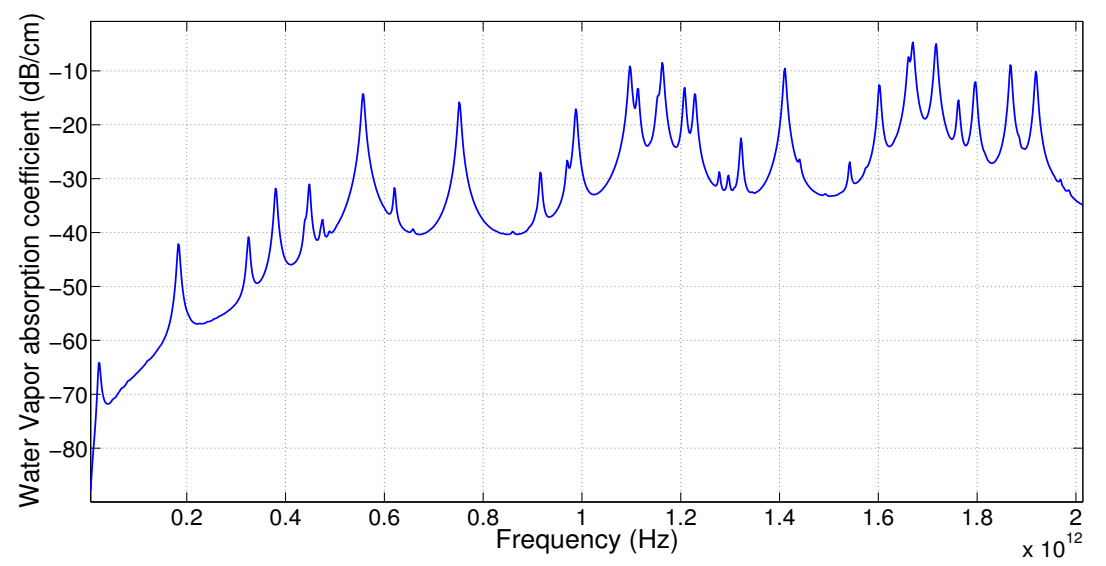

Figure 3.16: Water vapor absorption coefficient for $42 \%$ relative humidity in $\mathrm{dB} / \mathrm{cm}$.

\section{Calculating Oxygen Absorption Coefficient in our channel}

As we know, $20.946 \%$ of the air is oxygen. Therefore, the mixing ratio, $q$, of oxygen is equal to 0.20946 . Density of oxygen at $296 \mathrm{~K}$ is $1.308 \mathrm{gr} /$ Liter and since each gram of oxygen has 0.0625023438379 moles; therefore, the thickness, $u$, of oxygen is 0.081753 moles/Liter, which is $0.081753 \times 6.02214129 \times 10^{23}$ molecule/Liter or $4.923 \times 10^{19}$ molecule $/ \mathrm{cm}^{3}$. As previously explained, we use HITRAN database to 
extract the required parameters for oxygen absorption lines. Figure 3.17 shows the oxygen absorption coefficient in $\mathrm{dB} / \mathrm{cm}$.

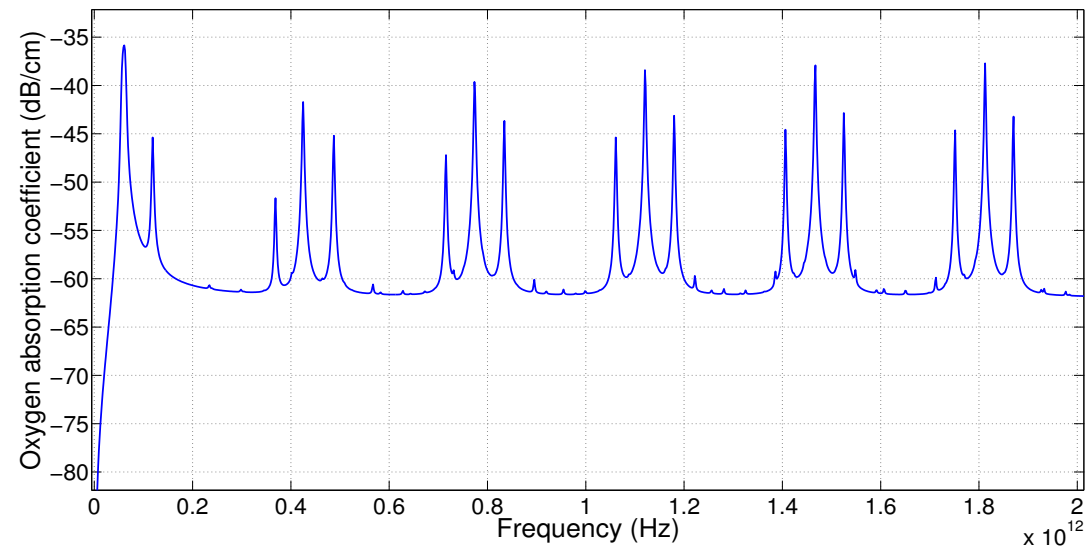

Figure 3.17: Oxygen absorption coefficient in $\mathrm{dB} / \mathrm{cm}$.

\section{Calculating Nitrogen Absorption Coefficient in our channel}

Nitrogen forms $78.084 \%$ of air. Therefore $q$ of nitrogen is equal to 0.78084 . Also, Nitrogen density is equal to $1.145 \mathrm{gr} / \mathrm{L}$ or 0.0817465 mole/Liter. Multiplying this value by Avogadro constant $\left(6.02214129 \times 10^{23}\right)$ gives the number of molecule, which is $4.9229 \times 10^{19}$ molecule $/ \mathrm{cm}^{3}$. Figure (3.18) shows the Nitrogen absorption coefficient in $\mathrm{dB} / \mathrm{cm}$. 


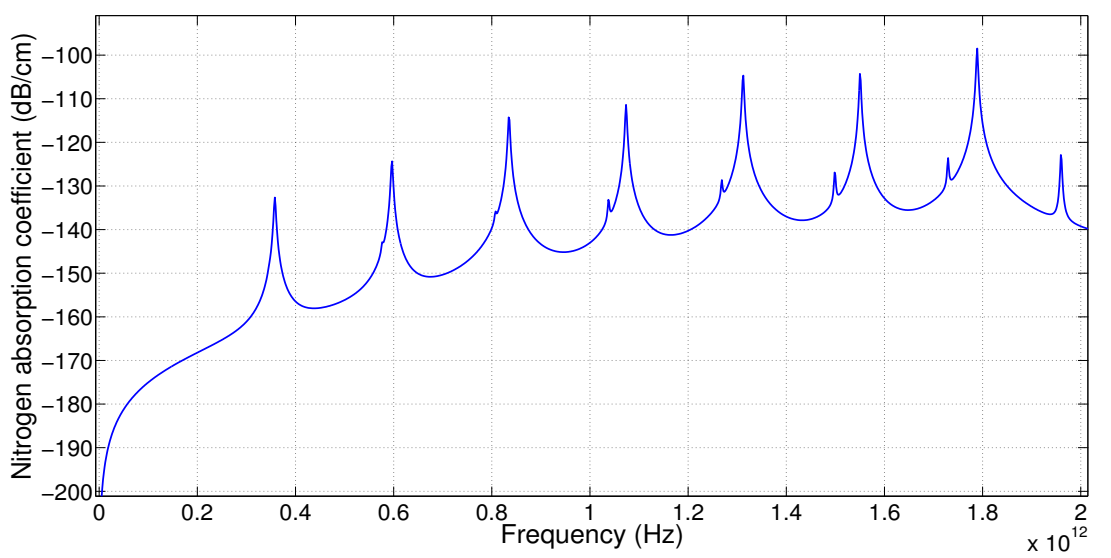

Figure 3.18: Nitrogen absorption coefficient in $\mathrm{dB} / \mathrm{cm}$.

\section{Calculating Carbon Dioxide Absorption Coefficient in our channel}

Carbon dioxide is only $0.035 \%$ of the air. As a result $q$ is equal to 0.00035 which is used in the (3.2) for calculating the Carbon dioxide half-width parameter. Carbon dioxide has density of $1.799 \mathrm{gr} / \mathrm{L}$ in $296 \mathrm{~K}$ or 0.040877 moles/Liter, which is $8.6159 \times 10^{15}$ molecule $/ \mathrm{cm}^{3}$. Figure(3.19) shows the Carbon dioxide absorption coefficient in $\mathrm{dB} / \mathrm{cm}$.

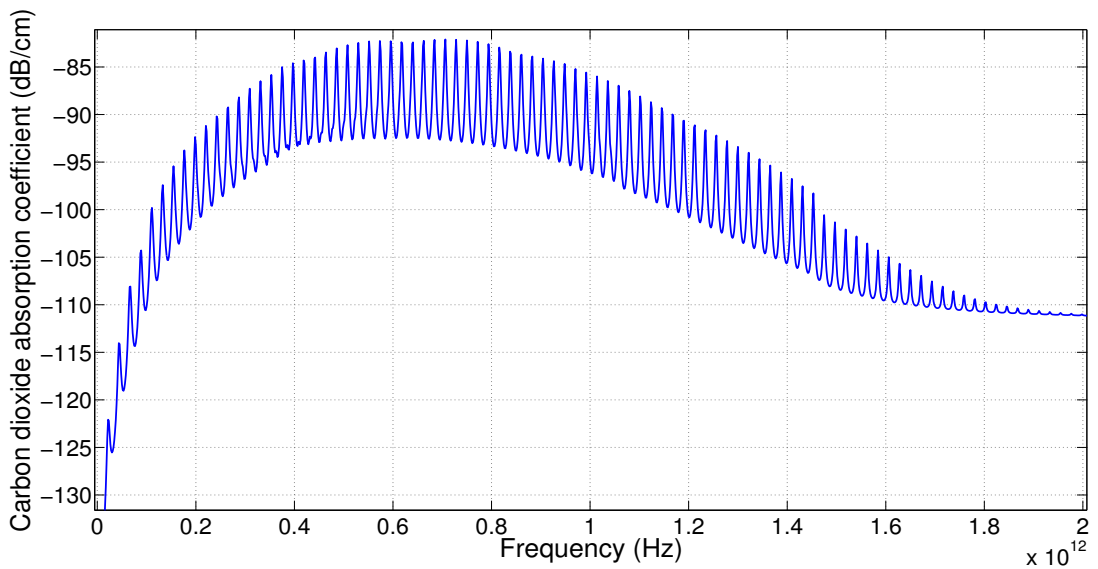

Figure 3.19: Carbon dioxide absorption coefficient in $\mathrm{dB} / \mathrm{cm}$. 


\section{Calculating Total Absorption Coefficient in our channel}

For calculating the total absorption coefficient, we need to add the calculated absorption coefficients corresponding to all molecules. Therefore, we calculate the total absorption coefficient for dry and humid channels from (3.10) and (3.11), respectively.

$$
\begin{gathered}
K_{\text {dry }}(\nu)\left(\mathrm{cm}^{-1}\right)=K_{\mathrm{O}}+K_{\mathrm{n}}+K_{\mathrm{C}} \\
K_{\text {humid }}(\nu)\left(\mathrm{cm}^{-1}\right)=K_{\mathrm{W}}+K_{\mathrm{O}}+K_{\mathrm{n}}+K_{\mathrm{C}}
\end{gathered}
$$

Where, $K_{\mathrm{W}}$ corresponds to water vapor absorption coefficient, $K_{\mathrm{O}}$ corresponds to oxygen absorption coefficient, $K_{\mathrm{n}}$ corresponds to nitrogen absorption coefficient, and $K_{\mathrm{C}}$ corresponds to carbon dioxide absorption coefficient.

We calculate the total absorption coefficient for different humidity levels. The Matlab code for calculating the atmospheric attenuation is in Appendix B. Figure 3.20 shows the result in $\mathrm{dB} / \mathrm{cm}$ for the range of $0-2 \mathrm{THz}$ for $0 \%, 10 \%, 40 \%$, and $80 \%$ RH levels.

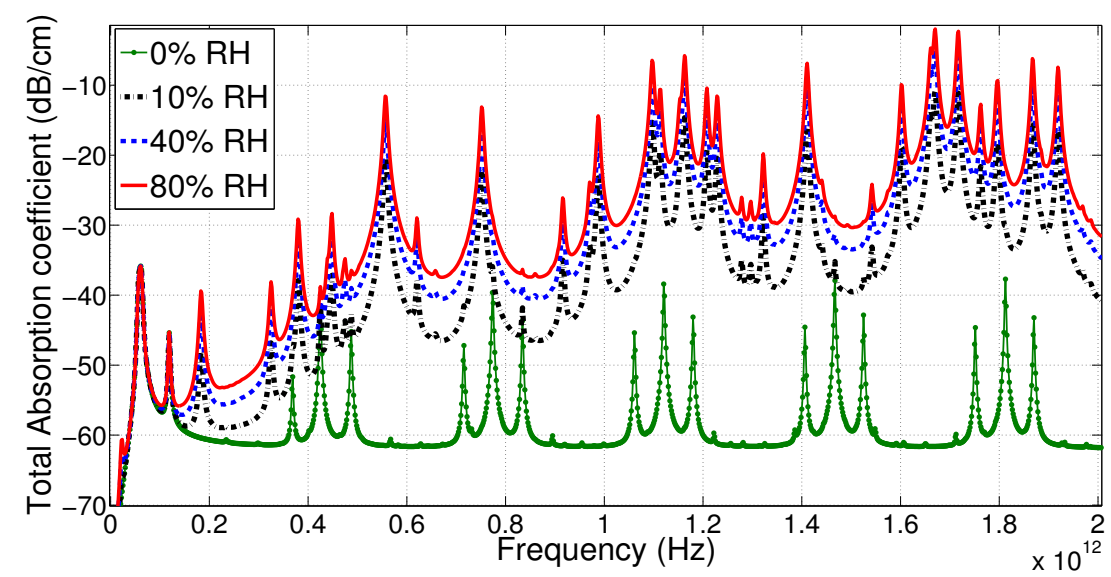

Figure 3.20: Atmospheric attenuation coefficient for different relative humidity $(\mathrm{RH})$ levels. 
As the figure shows and we expected, with higher humidity the attenuation coefficient is increased. The attenuation coefficient corresponding to some frequency bands is slightly attenuated due to the increase in humidity, whereas other frequencies are drastically attenuated. For example, in $400 \mathrm{GHz}$ band the signal power will be decreased $-45 \mathrm{~dB} / \mathrm{cm}$ when the $\mathrm{RH}$ is $40 \%$ and the corresponding number is $-60 \mathrm{~dB} / \mathrm{cm}$ for $0 \% \mathrm{RH}$. However, in $750 \mathrm{GHz}$ band the signal power will be decreased $-18 \mathrm{~dB} / \mathrm{cm}$ when the $\mathrm{RH}$ is $40 \%$ and the corresponding number for $0 \% \mathrm{RH}$ is $-60 \mathrm{~dB} / \mathrm{cm}$. This explains why we saw a smaller difference in received power for $42 \% \mathrm{RH}$ than $0 \% \mathrm{RH}$ in figures $3.10-3.12$ in comparison with the corresponding numbers in figures $3.13-3.15$.

\subsubsection{Modeling of Collimating Lenses Divergence}

Recall that we see a drop in received power at increasing distances even when $\mathrm{RH}$ is $0 \%$. We believe this is due to divergence in the beam. To model this, consider Figure 3.21, $d_{1}$ and $d_{2}$ are the transmitter-receiver distances for two different experiments, $\theta$ is the signal diverging angle and $r$ is the transmitter and receiver lenses' radius.

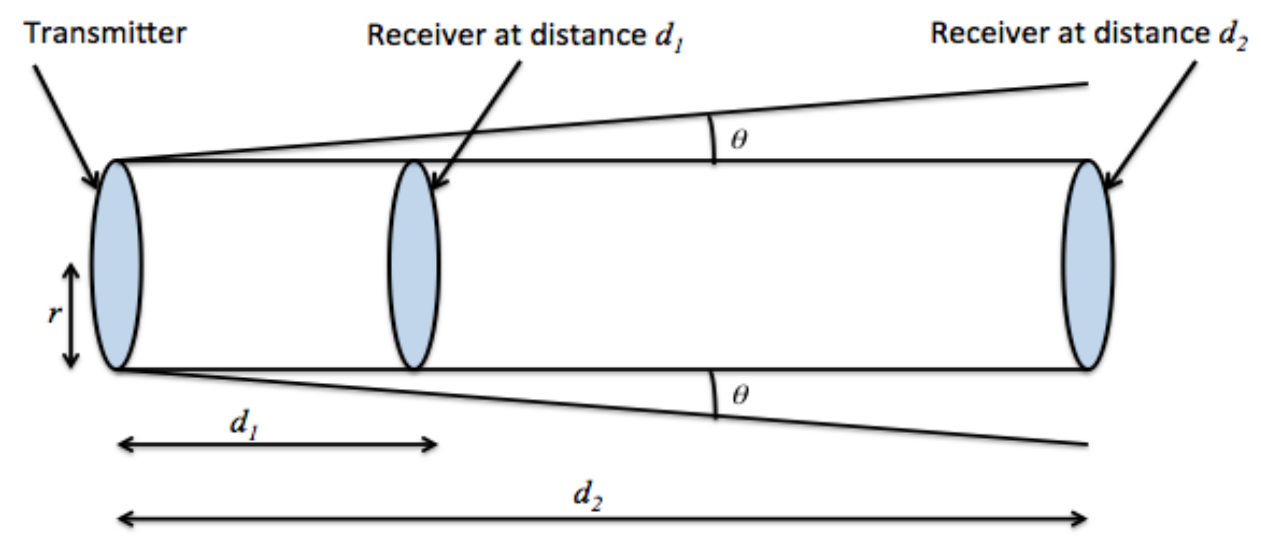

Figure 3.21: Diverging factor that causes attenuation in the received signal power. 
From the figure, since the signal is diverging with angle $\theta$, receiver receives $P_{t} \times \frac{\pi r^{2}}{\pi(r+d \tan \theta)^{2}}$ portion of the transmitted power, where $P_{t}$ is the signal power at the transmitter. Moreover, based on Friis transmission equation, the received signal power $P_{r}$ is proportional to the product of transmitted signal power $P_{t}$, transmitter gain $G_{t}$, receiver gain $G_{r}$ and the wavelength $\lambda$ as below.

$$
P_{r} \approx P_{t} G_{t} G_{r} \lambda^{2}
$$

Therefore, the received signal power for a specific frequency band at distance $d$ from transmitter can be calculated as below:

$$
P_{r}=\frac{\pi r^{2}}{\pi(r+d \tan \theta)^{2}} \times P_{t} G_{t} G_{r} \lambda^{2}
$$

Since $P_{t} G_{t} G_{r} \lambda^{2}$ is a constant value for each frequency band with wavelength $\lambda$, we can write the ratio of received power at transmitter-receiver distances $d_{1}$ and $d_{2}$ for a frequency band.

$$
\frac{P_{r_{d_{1}}}}{P_{r_{d_{2}}}}=\frac{\left(r+d_{2} \tan \theta\right)^{2}}{\left(r+d_{1} \tan \theta\right)^{2}}
$$

In (3.14), $P_{r_{d_{1}}}$ is the received power at distance $d_{1}$ and $P_{r_{d_{2}}}$ is the received power at distance $d_{2}$. From (3.14), we can find the signal diverging angle as below:

$$
\tan \theta=\frac{r\left(\sqrt{P_{r_{1}}}-\sqrt{P_{r_{1}}}\right)}{d_{2} \sqrt{P_{r_{d_{2}}}}-d_{1} \sqrt{P_{r_{d_{1}}}}}
$$

Experimental results. We repeat our experimental measurement 5 times for all distances in Table 3.1 for the directional channel. We chose $d_{1}$ as $11.5 \mathrm{~cm}$ and $d_{2}$ as $66.5 \mathrm{~cm}$. To find $\theta$, we first filter frequencies from 100 to $1000 \mathrm{GHz}$ into $10 \mathrm{GHz}$ bands using a raised cosine filter explained in 2.4.1, and find the diverging angle for all 5 repetitions. The Matlab code for the raised cosine filter is in Appendix C. We calculate the average diverging angle $\bar{\theta}$ and based on that we find the $95 \%$ 
confidence interval from the following equation.

$$
\left(\bar{\theta}-z_{1-\frac{\alpha}{2}} \frac{s}{\sqrt{n}}, \bar{\theta}+z_{1-\frac{\alpha}{2}} \frac{s}{\sqrt{n}}\right)
$$

Here $\bar{\theta}$ is the average value of the diverging angle for each filtered frequency band, $s$ is the sample standard deviation, $n$ is number of repetitions, and $z_{1-\frac{\alpha}{2}}$ is the $\left(1-\frac{\alpha}{2}\right)$-quantile of a unit normal variate. In our case we repeated the experiment 5 times for each transmitter-receiver distance. Hence, $n$ is equal to 5 and for $95 \%$ confidence level $z_{1-\frac{\alpha}{2}}$ is equal to 1.960 .

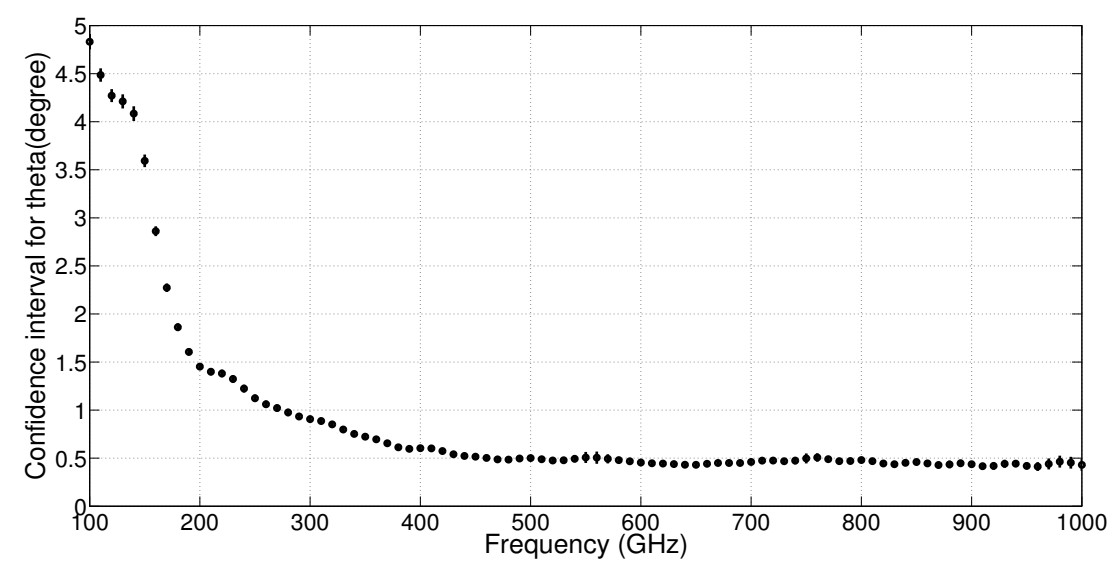

Figure 3.22: Confidence interval on the average value of diverging factor found from (3.15) for distances 11.5 and $66.5 \mathrm{~cm}$ when $\mathrm{RH}$ is 0.

Figure 3.22 shows that the value of the diverging angle is almost constant in all of our 5 experiments. For all cases, the diverging angle between $100 \mathrm{GHz}$ and $400 \mathrm{GHz}$ is not constant and decreases from $\sim 5^{\circ}$ to $0.5^{\circ}$. On the other hand, the diverging angle for bands between $400 \mathrm{GHz}$ to $1000 \mathrm{GHz}$ is constant and is around $0.4^{\circ}$. This behavior is because frequencies below $400 \mathrm{GHz}$ do not get fully collimated and are diffracted more than higher frequency components. Therefore, we get a bigger diverging angle for smaller frequencies [11]. 
Based on the value of $\theta$ from Figure 3.22 and the attenuation coefficient from Figure 3.20, we calculate the received power vs. transmitter-receiver distance for $400 \mathrm{GHz}$ frequency and 0\% RH. Figure 3.23 is the comparison of the simulation result and the experimental result from Figure 3.12.

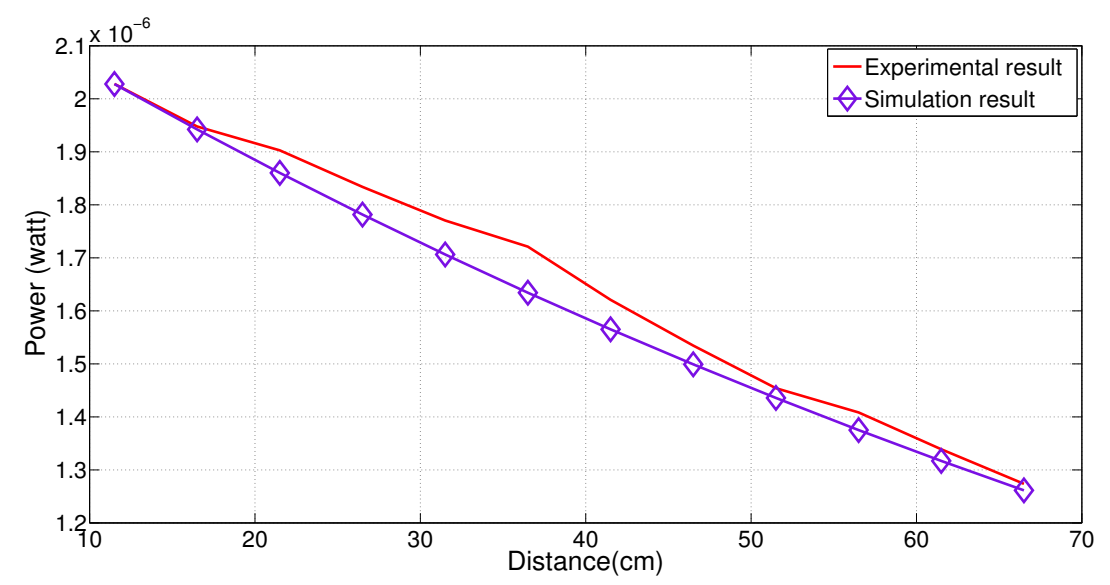

Figure 3.23: A comparison of the experimental and the simulation result of the received power in $400 \mathrm{GHz}$ frequency when $\mathrm{RH}$ is $0 \%$.

As the result show, the experimental and the simulation result are in a good agreement and both have the same decreasing slope.

\subsubsection{Summary of the Directional Channel Model}

We saw that the primary impairment to $\mathrm{THz}$ communications is the absorption of the transmitted signal by water vapor molecules in the channel. Also, we saw that the total attenuation coefficient is calculated from (3.11). Here we present the analytical formulation for obtaining the attenuation as a function of distance and humidity.

First from (3.1) we can calculate the corresponding wave number for each frequency and as a result we get $K(f)$, that is the total attenuation coefficient as a 
function of frequency. According to the Beer-Lambert law transmittance of the signal decreases exponentially with the transmitter-receiver distance [64]. Therefore, the transfer function of the channel is set to:

$$
H(f)=\frac{r^{2}}{(r+d \tan (\theta))^{2}} \times e^{-(j 2 \pi f \tau+d K(f))}
$$

Where, $\frac{r^{2}}{(r+d \tan (\theta))^{2}}$ corresponds to the diverging angle, $d$ is the transmitter-receiver distance, $\tau$ is the time in seconds required for the signal to travel through distance $d$, and $K(f)$ is the atmospheric attenuation from (3.11).

Having the transfer function from (3.17), we can characterize the channel as below:

$$
R(f)=S(f) H(f)+\text { Noise }
$$

Equation (3.18) shows the effect of the channel on the signal. The input signal, $S(f)$, is multiplied by the transfer function, $H(f)$, and Noise is added to the result and $R(f)$ that is the signal in frequency domain is received at the receiver. For Noise, we use a standard model and assume it is a random variable with a Gaussian distribution with power of $-174 \mathrm{dBm} / \mathrm{Hz}$. Based on the channel model from (3.18), we can find the received signal for a specific humidity and transmitterreceiver distance.

Figure 3.24 compares experimental measurements with our simulation results for a 51.5-cm transmitter-receiver distance and 0\% RH. Simulation results, shown as a solid line, are calculated from our model given above and the experimental results are illustrated by the dotted line. As can be observed from this figure, the measurement results are in close agreement with values calculated from our proposed model especially at high frequencies.

Similarly, Figure 3.25 compares experimental and theoretical results for $42 \%$ RH. Again, it can be clearly observed from the figure that measured and calculated 


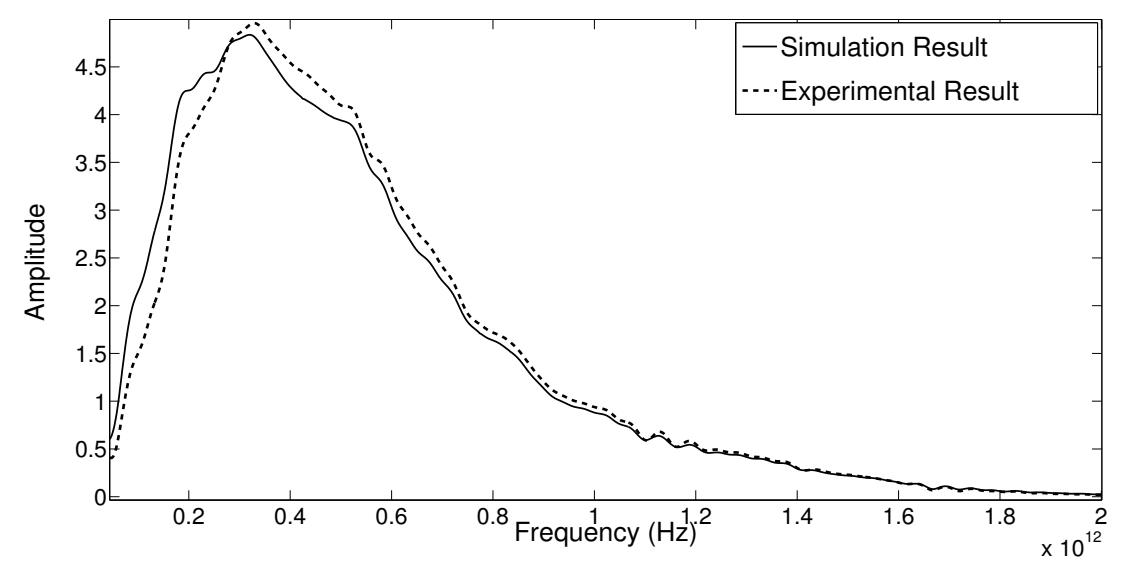

Figure 3.24: Simulated vs Experimental signal over $50 \mathrm{~cm}$ distance and $0 \% \mathrm{RH}$. Solid and dotted line represent simulation and experimental results, respectively.

numbers are very similar. The dips we see in the figure correspond to water and other absorption lines.

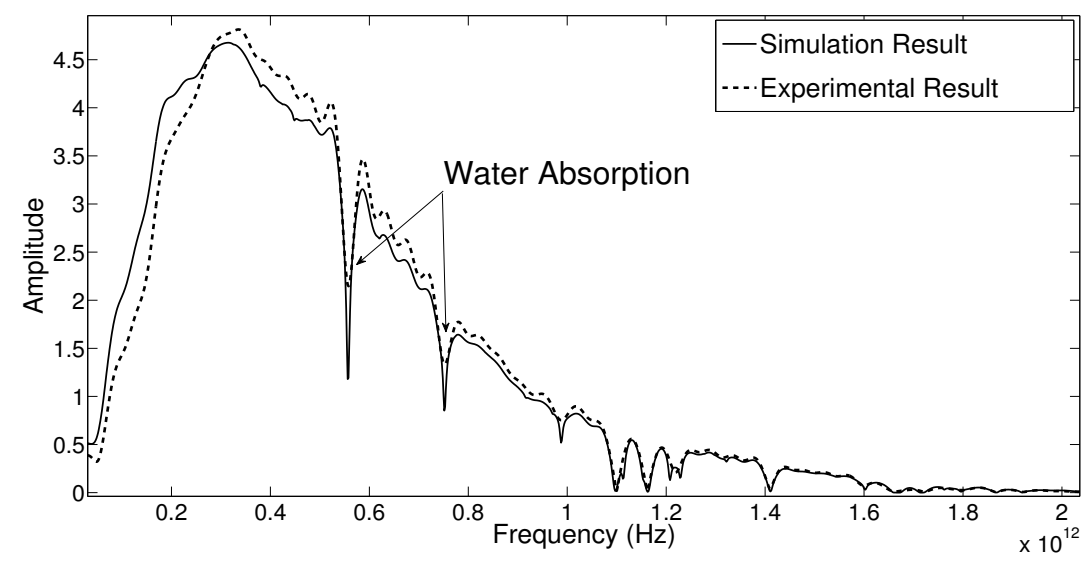

Figure 3.25: Simulated vs Experimental signal over $50 \mathrm{~cm}$ distance and $42 \% \mathrm{RH}$. Solid and dotted line represent simulation and experimental results, respectively. 


\section{NON-DIRECTIONAL CHANNEL CHARACTERIZATION}

In the non-directional channel case we remove the collimating lenses from both the transmitter and receiver. Figure 3.26 shows the received power for different transmitter-receiver distances when the humidity is $42 \%$. In this case the transmitted beams are not parallel and as a result the received signal attenuates more as transmitter-receiver distance increases in comparison with the directional case in Figure 3.8.

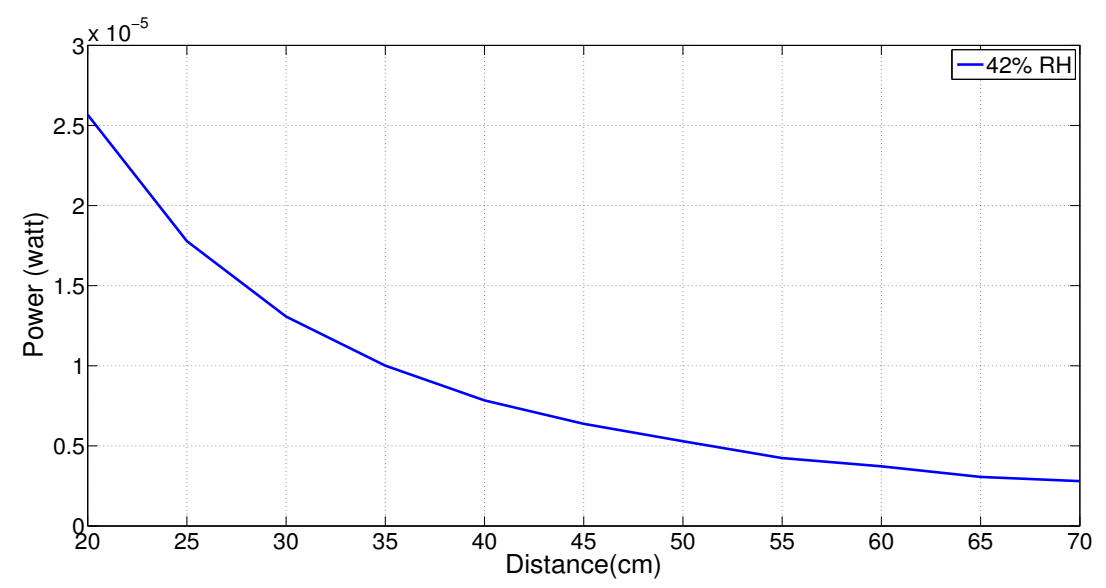

Figure 3.26: Received power vs. transmitter-receiver distance in non-directional channel when humidity is $42 \%$.

Figure 3.27 shows the frequency spectrum of the signal for the non-directional channel when the transmitter-receiver distance is $50 \mathrm{~cm}$. 


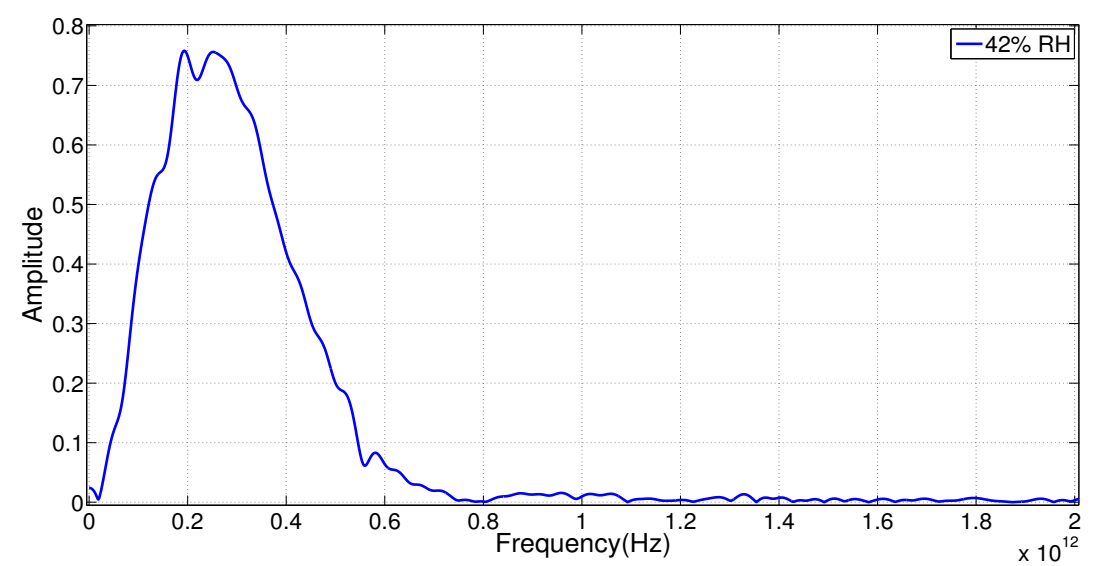

Figure 3.27: Frequency spectrum of the signal in non-directional channel with transmitter-receiver distance of $50 \mathrm{~cm}$ and $42 \% \mathrm{RH}$.

As we see, the receiver only receives part of the signal that has frequency below $700 \mathrm{GHz}$ and the higher frequency components are gone. This happens due to the fact that higher frequencies have smaller wavelengths and as a result they are attenuated more with distance.

From the result from Figure 3.26, we find a function that fits the experimental received power using Matlab cftool. The function we found is as below.

$$
P_{r}=\left(5.739 \times 10^{-5} d+0.01136\right) /\left(d^{2}+4.01 d+1.754\right)
$$

where, $P_{r}$ corresponds to the received power and $d$ is the transmitter-receiver distance. Figure 3.28 shows the result of fitting the experimental received power in Figure 3.26 with the function from (3.19). 


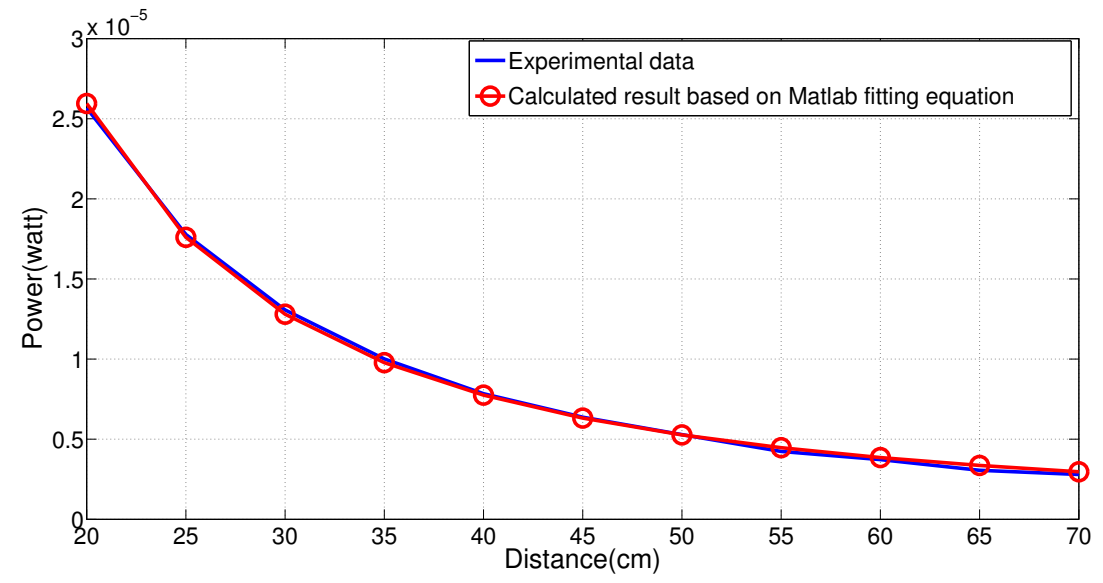

Figure 3.28: Experimental received power and the fitting function using Matlab "cftool".

In the figure, the solid line corresponds to the experimental received power and the line with circles corresponds to the function from (3.19). The figure shows that the result from the function we found is in a good agreement with the experimental result.

Equation (3.19) can be broken into two parts as below.

$$
\begin{gathered}
P_{r 1}=5.739 \times 10^{-5} d /\left(d^{2}+4.01 d+1.754\right) \\
P_{r 2}=0.01136 /\left(d^{2}+4.01 d+1.754\right)
\end{gathered}
$$

where, $P_{r}=P_{r 1}+P_{r 2}$. Figure 3.29 shows the result of fitting only the second part of the function with the experimental result. 


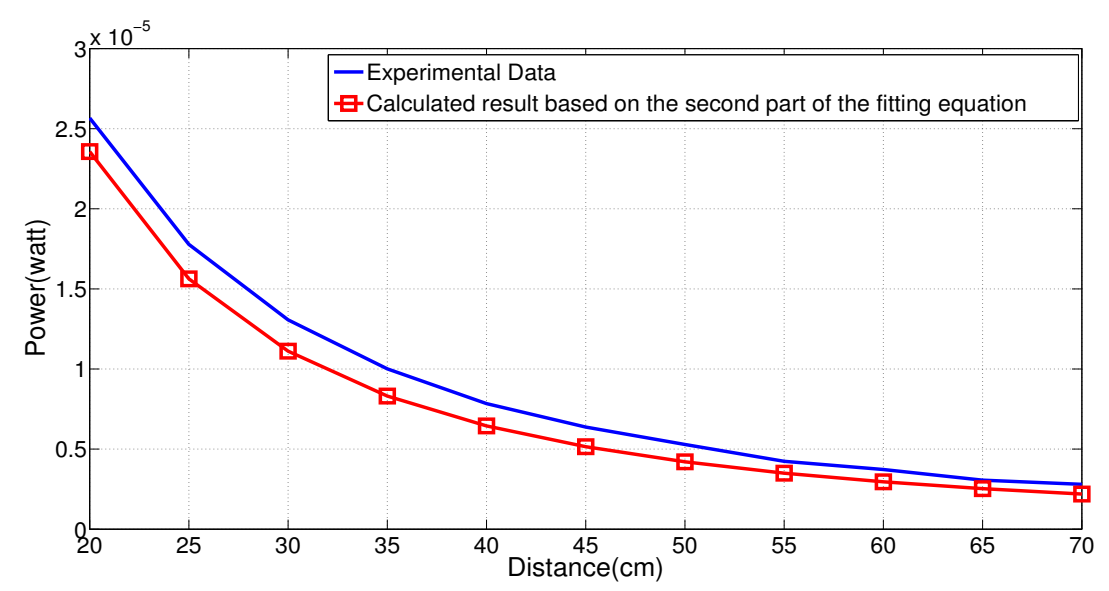

Figure 3.29: Curve fitting with the second part of the (3.19) .

The fitting function has lower degree than Friis formula. Friis equation that corresponds to omni-directional channels is as below:

$$
P_{r}=C \times \frac{1}{d^{2}} \times P_{t}
$$

Here, $P_{t}$ is the transmitted power, and $C$ is a constant factor that is calculated based on values such as transmitter and receiver antenna gains and wavelength of the frequency. In Friis equation the numerator degree is 0 while the denominator degree is 2. However, we have a different equation in (3.19) that has degree of 1 in numerator and degree of 2 in denominator. Therefore, we conclude that if we remove the collimating lens from transmitter and receiver, the antenna of the Picometrix T-Ray system that we use is not omni-directional.

Since we have a large bandwidth, we investigate the fitting function for some individual bands as well. For that we consider the 3 frequency bands of $110 \mathrm{GHz}$, $170 \mathrm{GHz}$, and $400 \mathrm{GHz}$. Figures 3.30-3.32 show the experimental received power for each of these bands along with the fitting function for each of them. 


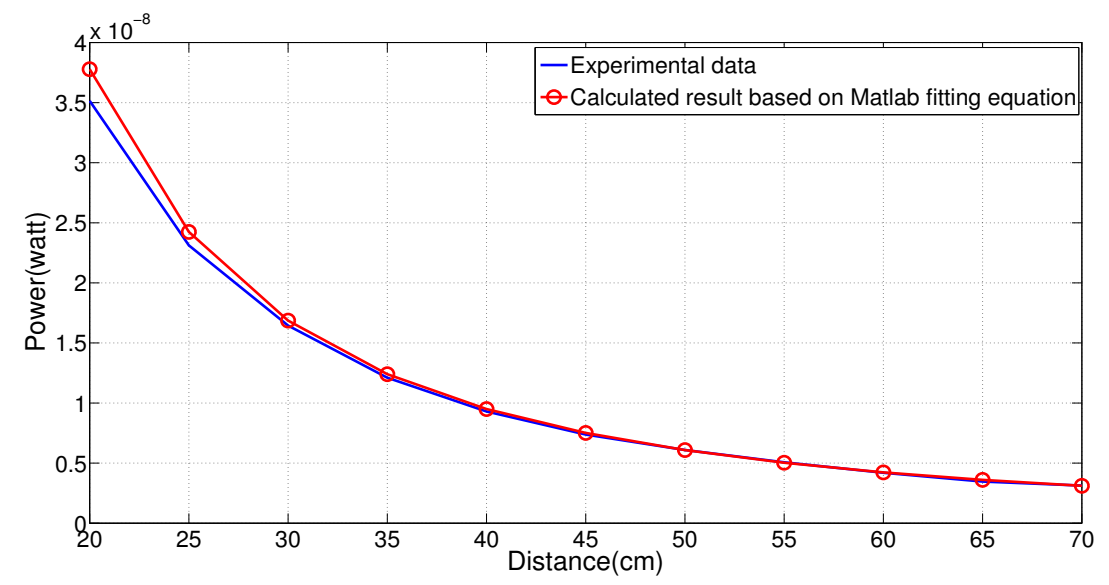

Figure 3.30: Experimental received power for $110 \mathrm{GHz}$ band along with the fitting function.

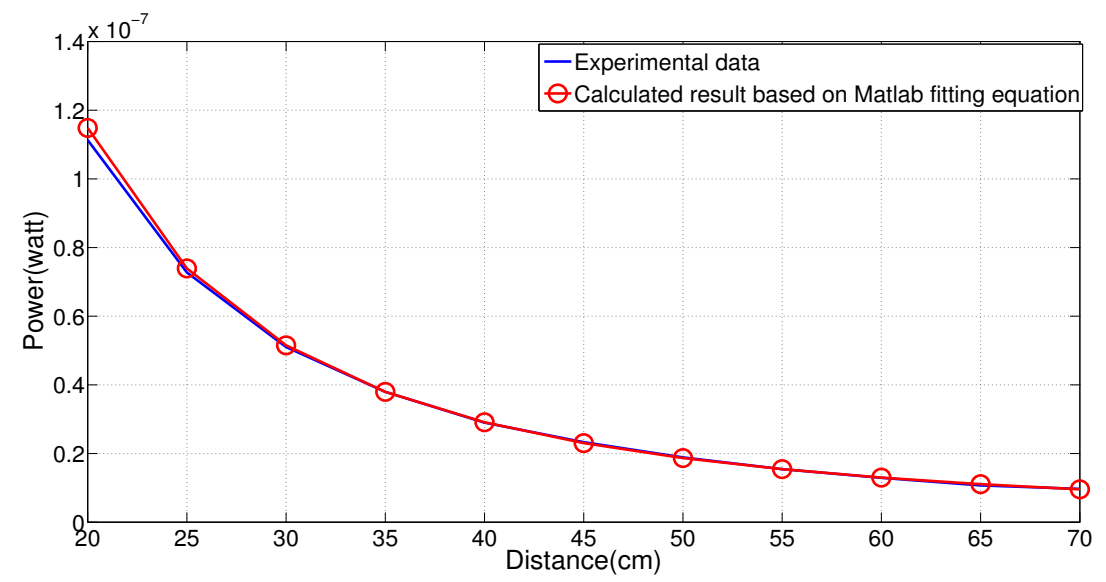

Figure 3.31: Experimental received power for $170 \mathrm{GHz}$ band along with the fitting function. 


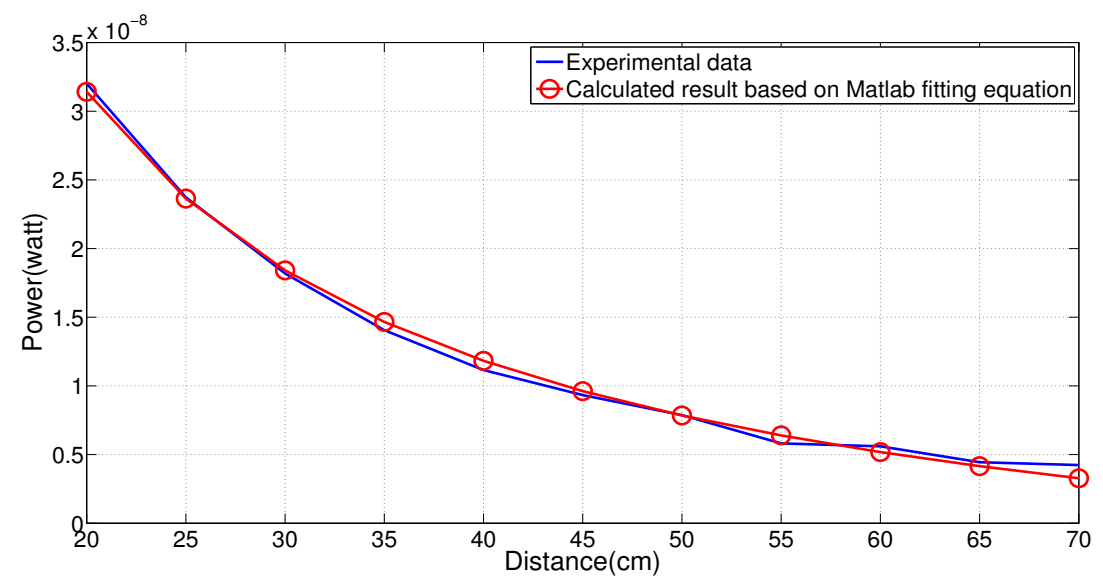

Figure 3.32: Experimental received power for 400GHz band along with the fitting function.

The following equations show the fitting functions for $110 \mathrm{GHz}, 170 \mathrm{GHz}$, and $400 \mathrm{GHz}$ bands, respectively.

$$
\begin{gathered}
P_{r}=\left(1.527 \times 10^{-5}\right) /\left(d^{2}+0.1576 d+0.9711\right) \\
P_{r}=\left(4.719 \times 10^{-5}\right) /\left(d^{2}+0.4981 d+0.9693\right) \\
P_{r}=\left(-8.283 \times 10^{-9} d+8.106 \times 10^{-7}\right) /(d+0.528)
\end{gathered}
$$

\subsubsection{Antenna Characterization}

As noted before, the antenna used in Picometrix 4000 T-Ray system is not omnidirectional. The goal of the analysis in this section is to characterize this antenna.

In Picometrix system a femtosecond laser generates $\mathrm{THz}$ pulses, then a photoconductive antenna (PCA) picks up these pulses. The main part of the PCA is a bowtie antenna with a photoconductive gap. When the antenna is illuminated by femtosecond laser pulses, pulsed current flows through the metallic part of the antenna. This radiation is then collimated by a high resistivity hemispherical silicon 
lens. A High-Density PolyEthylene (HDPE) lens is then used to further collimate the radiation or to focus it at some known distance from the transmitter. Figure 3.33 shows the structure of the PCA and Table 3.3 gives more specifications about the size and the material that is used in the PCA.

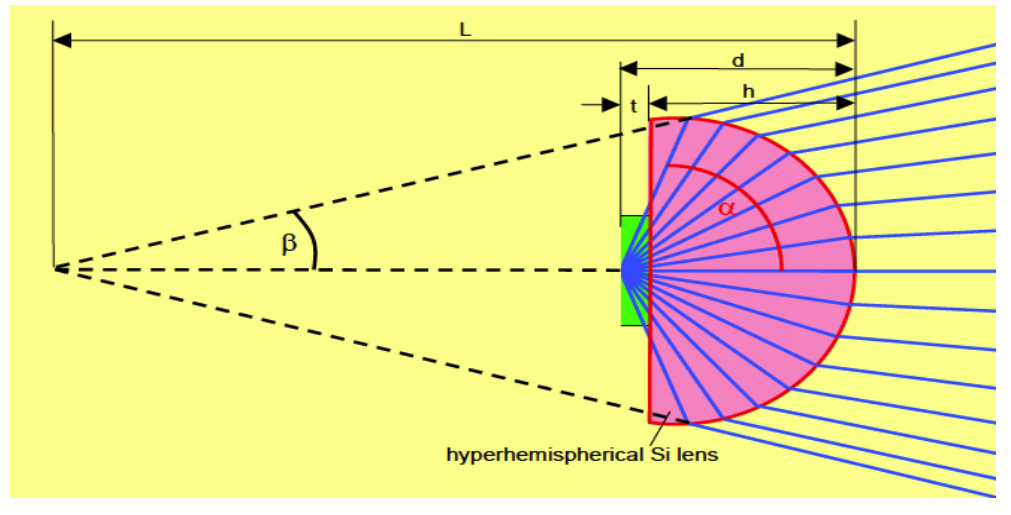

Figure 3.33: Antenna PCA structure [1].

Table 3.3: PCA specifications [1].

\begin{tabular}{|l|l|l|}
\hline \multirow{2}{*}{ Photoconductive antenna } & substrate & semi-insulating GaAs \\
chip area & $4 \mathrm{~mm} \times 4 \mathrm{~mm}$ \\
& thickness $t$ & $650 \mu \mathrm{m}$ \\
& active area & $1 \mathrm{~mm} \times 1 \mathrm{~mm}$ \\
\hline \multirow{2}{*}{ Hyperhemispherical lens } & material & undoped HRFZ-silicon \\
& specific resistance $\rho$ & $>10 \mathrm{k} \Omega \mathrm{cm}$ \\
refractive index $n$ & 3.4 \\
\hline Terahertz beam & height $h$ & $12 \mathrm{~mm}$ \\
& distance $d$ & $7.1 \mathrm{~mm}$ \\
\hline & collection angle $\alpha$ & $7.7 \mathrm{~mm}$ \\
\hline & divergence angle $\beta$ & $15^{\circ}$ \\
\hline & virtual focus length $L$ & $26.4 \mathrm{~mm}$ \\
\hline
\end{tabular}


Figure 3.34 shows the PCA that is used in the transmitter without the HDPE lens, and Figure 3.35 shows the transmitter with a collimating HDPE lens.

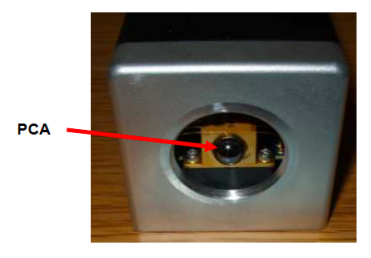

Figure 3.34: Picotemrix transmitter with PCA.

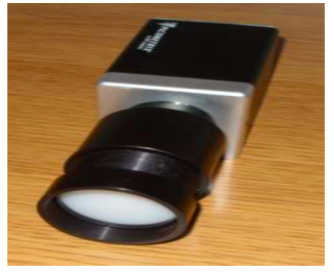

Figure 3.35: Picotemrix transmitter with PCA and HDPA lens.

\section{Experimental Measurement}

For the experimental measurement of the radiation pattern, we build a device that has a stable part connected to a moveable arm. We put the transmitter on the table just next to the stable part of the device and the receiver is mounted on the moveable arm. Therefore, the receiver can see different angles with respect to the transmitter. We remove the lens from the transmitter so that it only has the PCA. For the receiver we do the experiment once with collimating HDPA lens and once without it. Figure 3.36 shows the lab setup.

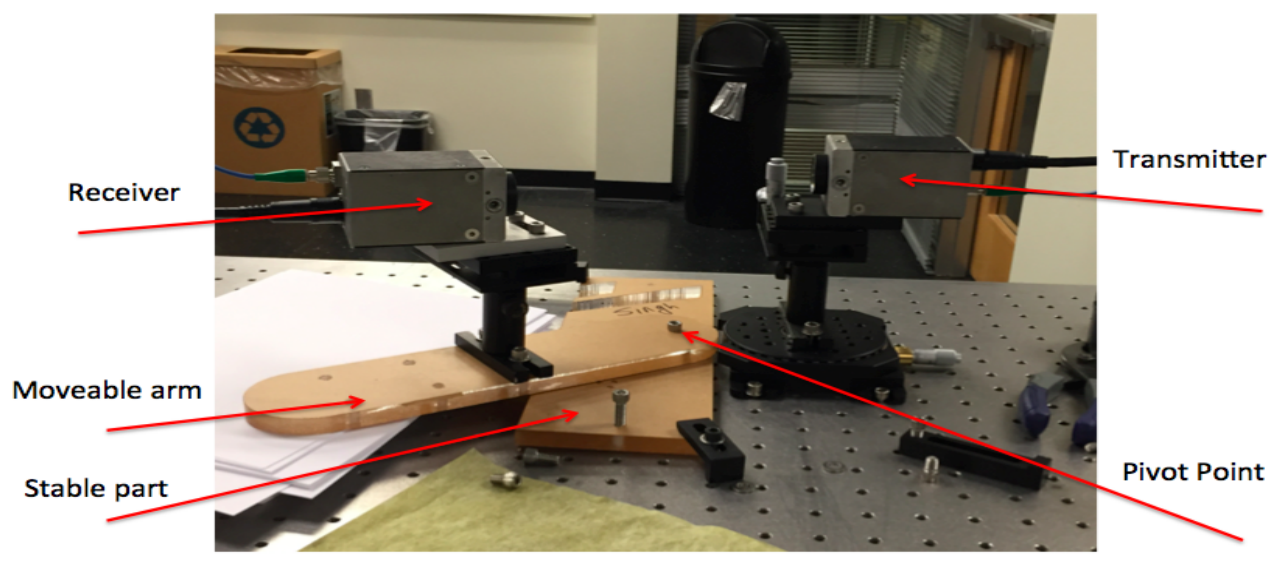

Figure 3.36: Lab setup for characterizing the antenna. 
In our measurements, we change the moveable arm such that the receiver sees angles of 0 to 40 degrees with increments of 5 degrees with respect to the pivot point.

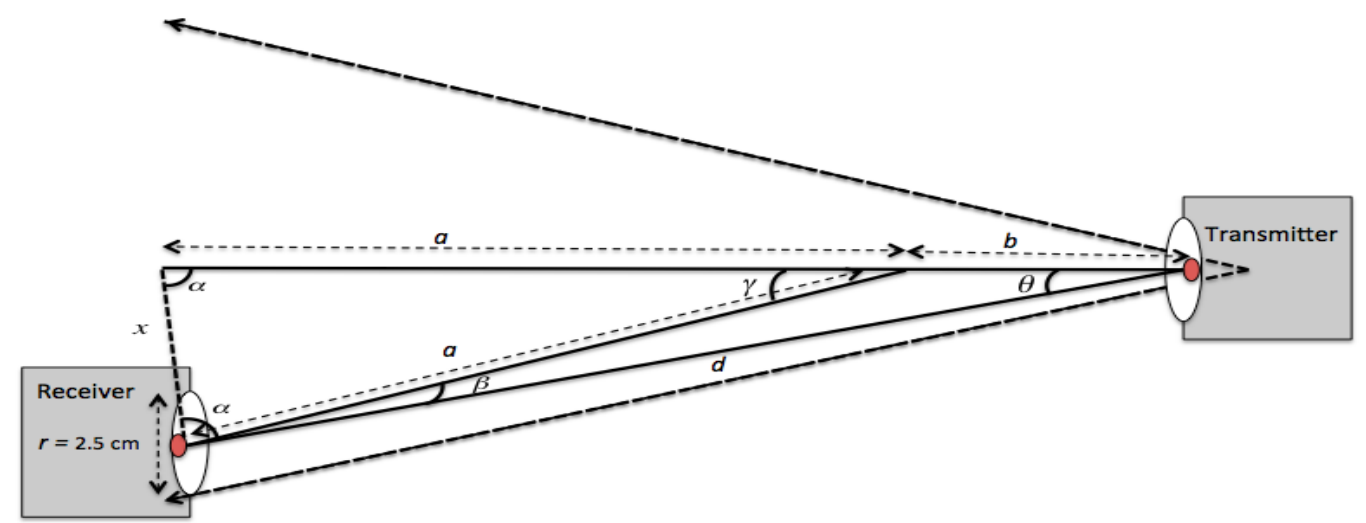

Figure 3.37: transmitter-receiver angle.

As we see in Figure 3.37, we have the value of $\gamma$, but we need to know the value of $\theta$ that is the actual transmitter-receiver angle. For that, we use the following equations to calculate the value of $\theta$ and the transmitter-receiver distance $d$.

$$
\begin{gathered}
x=2 a \cos (\alpha) \\
d=\sqrt{(a+b)^{2}+x^{2}-2 x(a+b) \cos (\alpha)} \\
\sin (\alpha+\beta)=(a+b) \frac{\sin (\alpha)}{d} \\
\theta=180-\alpha-(\alpha+\beta)
\end{gathered}
$$

For each transmitter-receiver angle $\theta$, we calculate the received power. Figure 3.38 shows the result for the case where receiver has a collimating lens. The numbers on the figure are the transmitter-receiver distances, $d$, in $\mathrm{cm}$. 


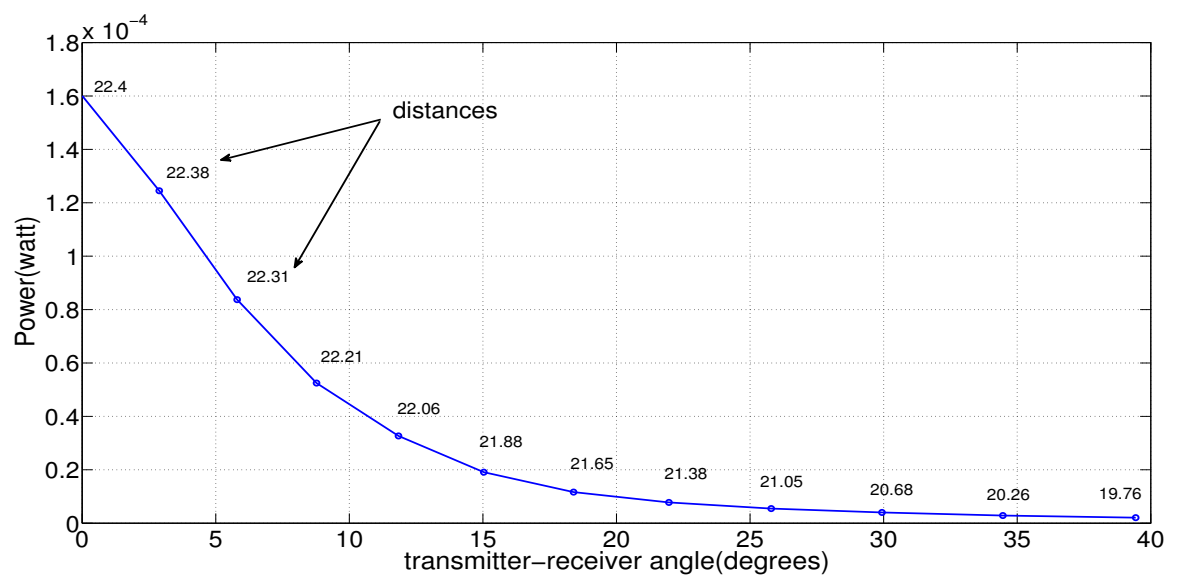

Figure 3.38: Received power in watt when receiver has collimating lens.

Figure 3.39 is the received power vs. transmitter-receiver distance for the case where we remove the collimating lens from the receiver.

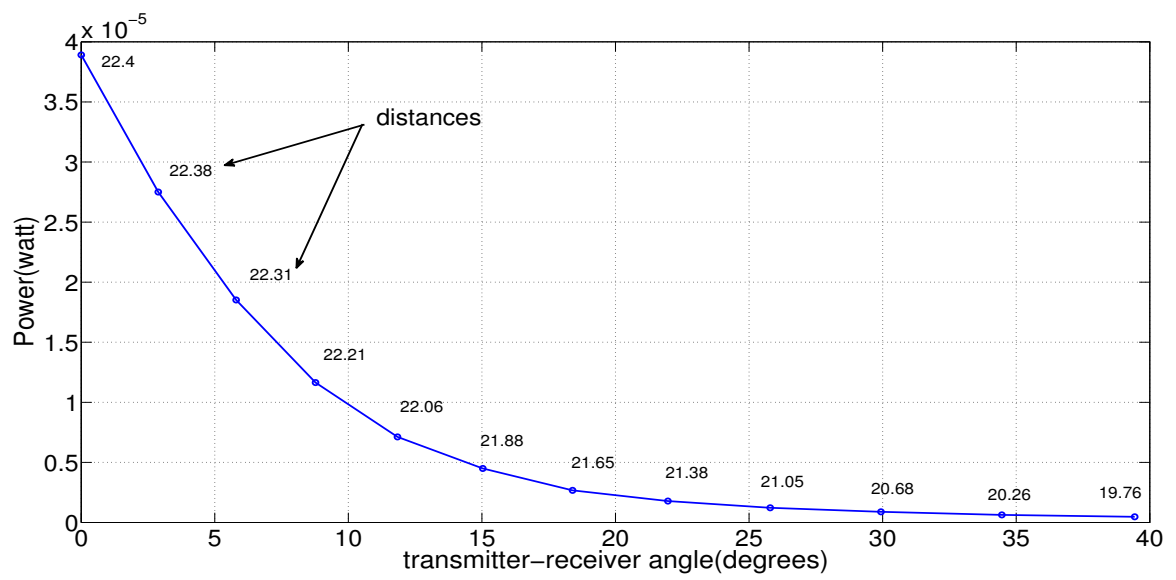

Figure 3.39: Received power in watt when receiver has no collimating lens.

The results of both figures show that the received power is decreased dramatically when transmitter-receiver angle is increased. For example, around transmitterreceiver angle of $6^{\circ}$ the power is almost half of the initial value.

To better understand the effect of the antenna on different frequencies, we 
calculate the received power only for frequency component of $300 \mathrm{GHz}$ with bandwidth of $1 \mathrm{GHz}$. Figures 3.40 and 3.41 show the result for cases when the receiver has a collimating lens and when it doesn't have a lens, respectively.

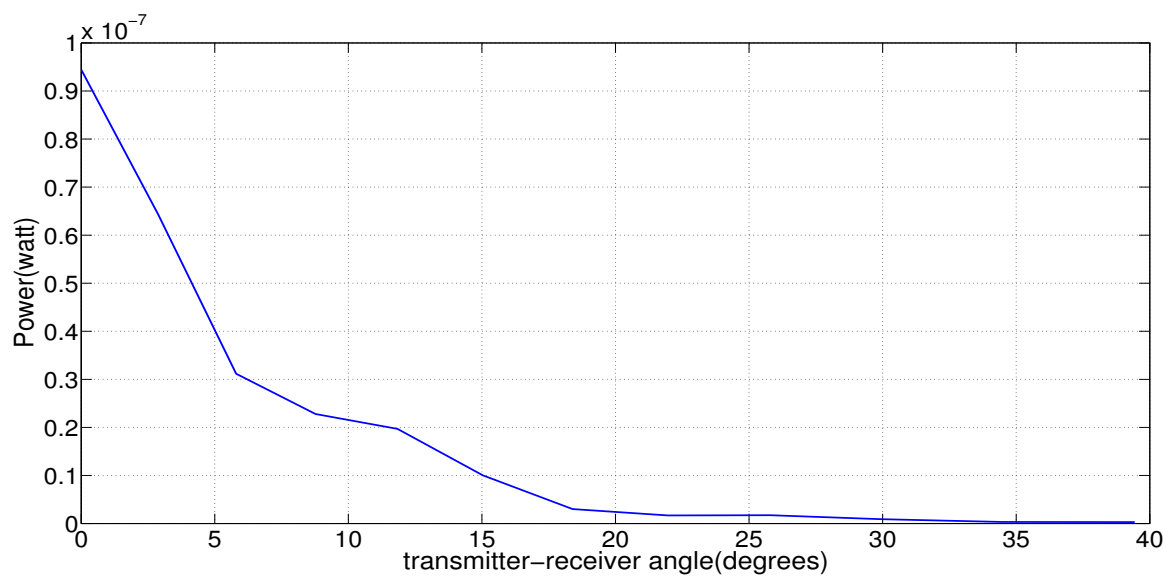

Figure 3.40: Received power in watt for $300 \mathrm{GHz}$ frequency band when receiver has collimating lens.

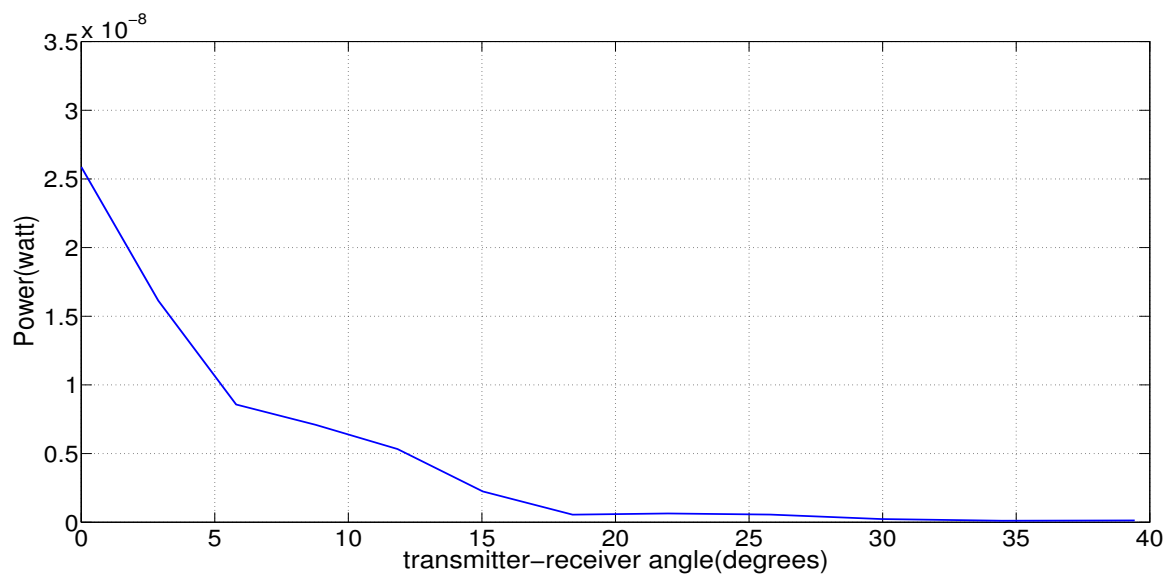

Figure 3.41: Received power in watt for $300 \mathrm{GHz}$ frequency band when receiver has no collimating lens.

For frequency component of $300 \mathrm{GHz}$, the power is halved even with transmitterreceiver angles less than $6^{\circ}$ as we saw earlier. 
To better understand the antenna characterization, we conduct other measurements to see if we get any different result for the other polarization plane. For that we rotate the transmitter and receiver heads by 90 degrees and we do the same measurements. Figure 3.42 and 3.43 is the received power for the receiver with collimating lens and receiver with no lens, respectively.

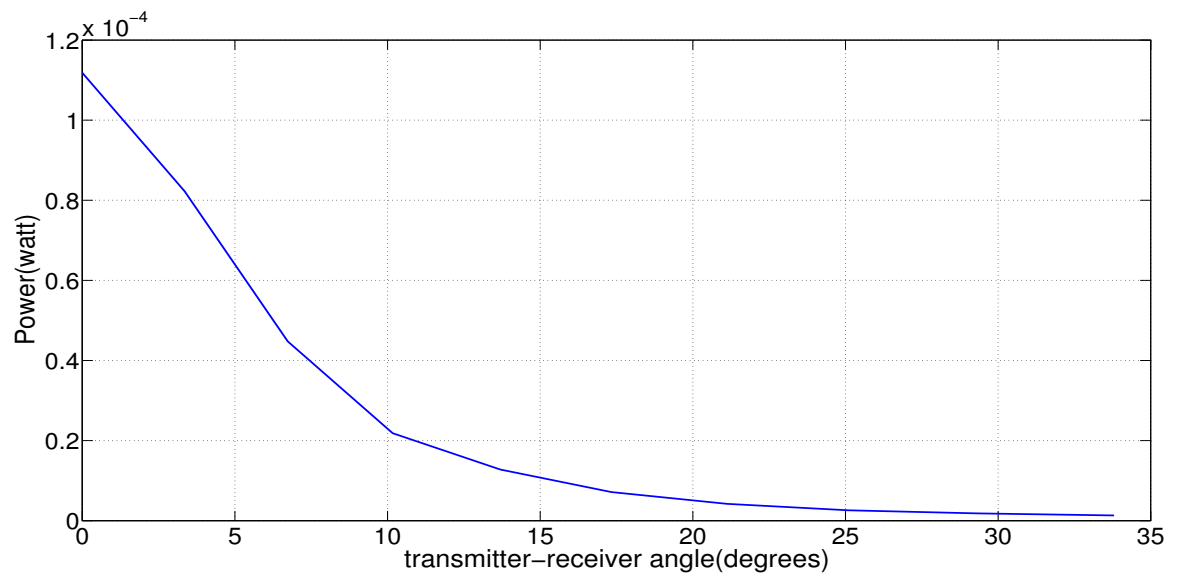

Figure 3.42: Received power in watt when receiver has collimating lens and transmitter and receiver heads are rotated $90^{\circ}$.

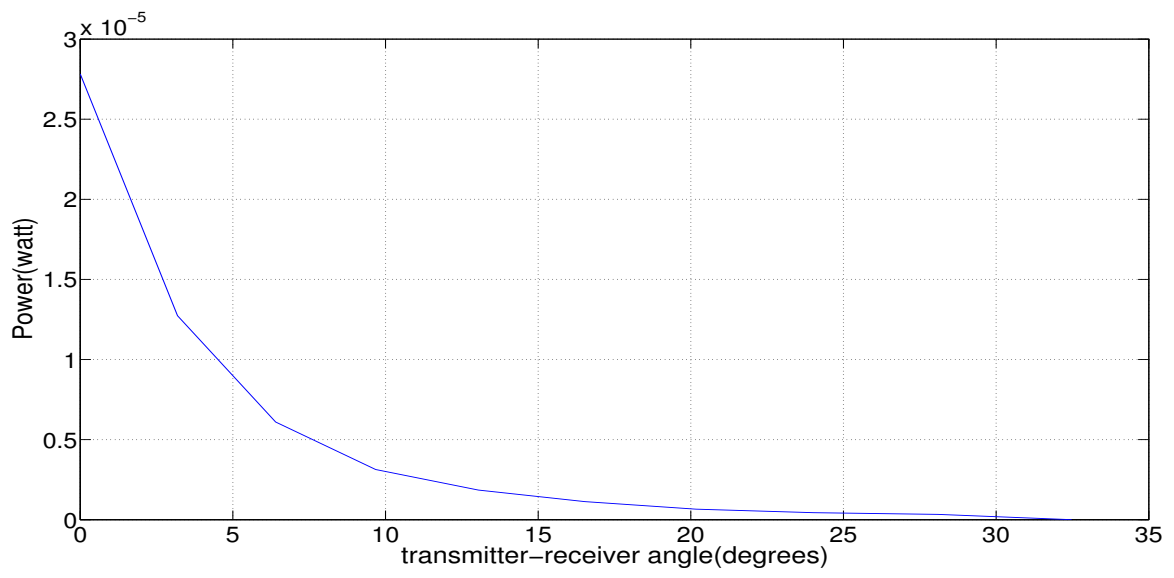

Figure 3.43: Received power in watt when receiver has no collimating lens transmitter and receiver heads are rotated $90^{\circ}$. 
As we saw earlier, the result of these figures show the same dramatic decrease in the received power as the transmitter-receiver angle is increased.

Figures 3.44 and 3.45 are the results for $300 \mathrm{GHz}$ frequency component when the receiver has a collimating lens and when it does not have a lens, respectively.

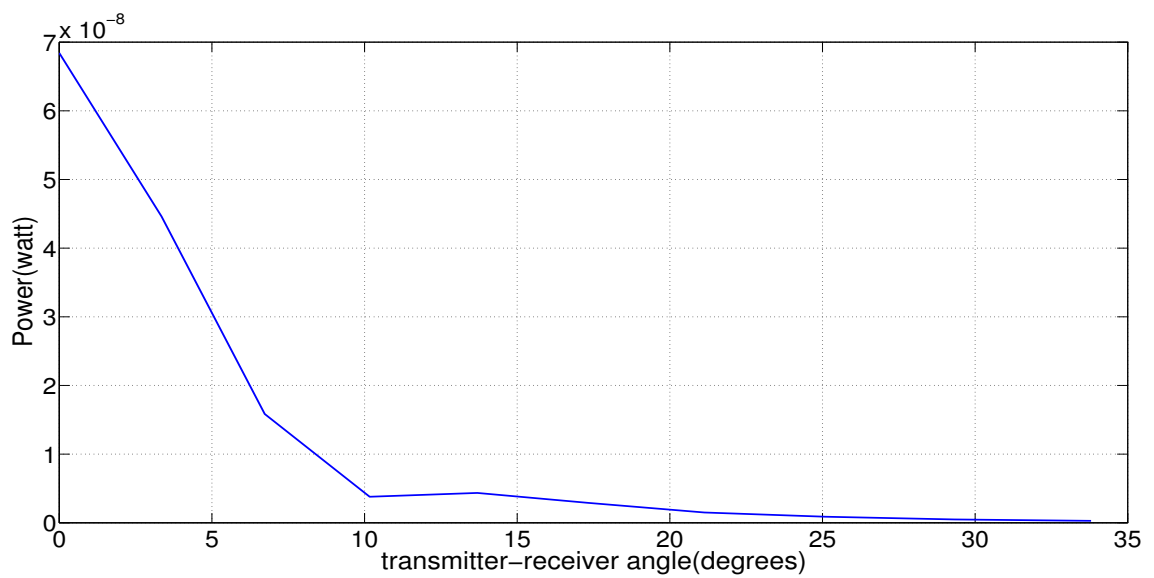

Figure 3.44: Received power in watt for $300 \mathrm{GHz}$ band when receiver has collimating lens and transmitter and receiver are rotated $90^{\circ}$.

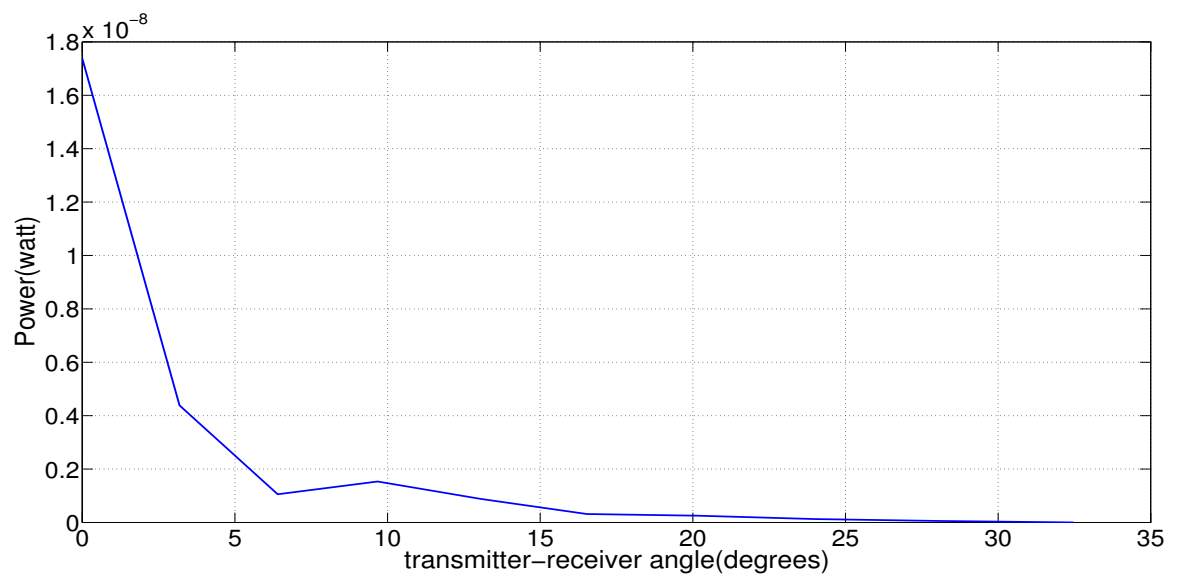

Figure 3.45: Received power in watt for $300 \mathrm{GHz}$ band when receiver has no collimating lens transmitter and receiver are rotated $90^{\circ}$.

For $300 \mathrm{GHz}$ frequency band the power is decreased drastically as before. 
Therefore, we conclude that the received power in both polarization planes is decreased with the same pattern and there is no difference in polarization planes.

\section{Airy Disk Effect}

The transmitter has a circular aperture and as a result the intensity of the received power can be explained by the airy disk effect [59, p. 283-291]. Airy disk is a description of the focus spot of a signal that has a circular aperture. The following equation explains the received power's intensity,

$$
I(\theta)=I_{0}\left(\frac{2 J_{1}(k r \sin \theta)}{k r \sin \theta}\right)^{2}
$$

where, $\theta$ is the angle between transmitter-receiver in degrees, $I_{0}$ is the received power with transmitter-receiver angle of $0, I_{\theta}$ is the received power with transmitterreceiver angle of $\theta, r$ is the radius of the circular aperture, $k$ is equal to $2 \pi / \lambda$ where $\lambda$ is the wavelength of the frequency, and $J_{1}$ is the Bessel function of the first kind that can be found from (3.31).

$$
J_{1}(x)=\sum_{m=0}^{\infty} \frac{(-1)^{m}}{m ! \Gamma(m+2)}\left(\frac{x}{2}\right)^{2 m+1}
$$

Here, $\Gamma(n)$ is the gamma function and for positive integers is defined as below.

$$
\Gamma(n)=(n-1) !
$$

We find the normalized received power based on (3.30) for frequency band of $300 \mathrm{GHz}$ and compare the result with the normalized power found from our lab measurements when the receiver has a collimating lens. Figure 3.46 shows the result. As we see, for a transmitter aperture radius, $r$, of $4 \mathrm{~mm}$ we get a good agreement between the result from (3.30) and our lab measurements. Note that 
the aperture size of $4 \mathrm{~mm}$ is the photoconductive antenna size that we saw in Table 3.3.

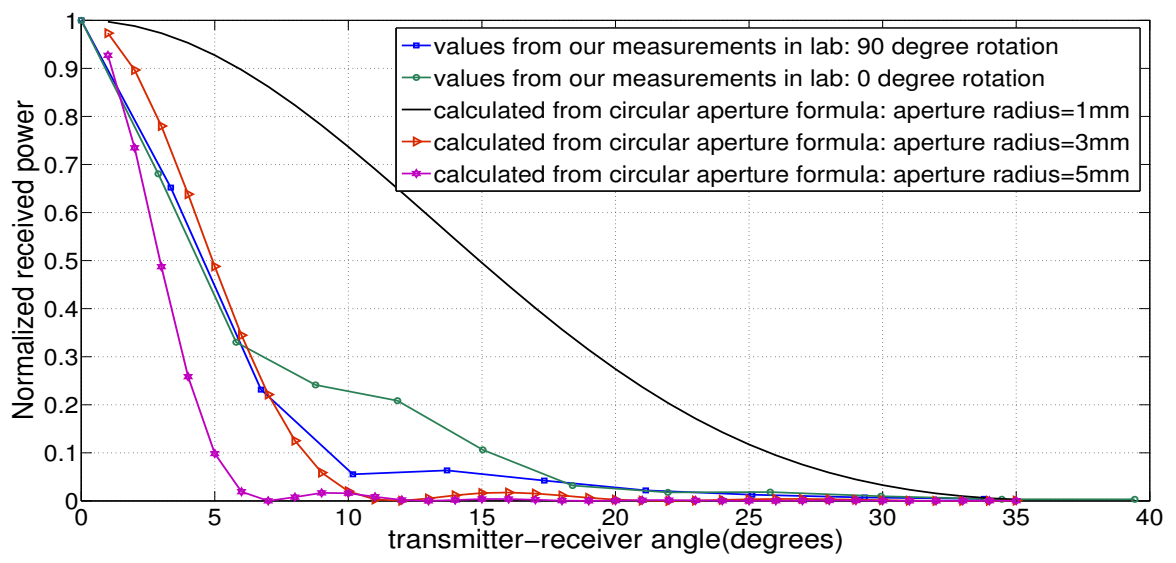

Figure 3.46: Comparison of the normalized received power for $300 \mathrm{GHz}$ band from (3.30) with the lab measurements when receiver has collimating lens.

\subsubsection{Summary of the Non-Directional Channel Model}

Our analysis shows that the received power of the Picometix T-Ray 4000 system that does not have collimating lens is decreased drastically as transmitter-receiver angle is increased. That is the received power is decreased to half when transmitterreceiver angle is increased from 0 degrees to 5 degrees. This result is in agreement with (3.19) that shows the Picometrix antenna is not an omni-directional antenna but rather has behavior that can be described by the airy disk effect.

\section{SUMMARY}

In this section, we analyzed the received signals in directional and non-directional channels. Also, we modeled the signal attenuation caused by atmospheric molecules for the $\mathrm{THz}$ frequencies. In the next chapters, we will use these concepts in our simulations to modulate, send, and receive data in our communication channels. 
Chapter 4

\section{DIRECTIONAL COMMUNICATION MODELS}

In this chapter and the next we study the problem of communicating using terahertz frequencies. Since there are no terahertz communication devices available, we use simulations with the channel model derived from our measurements in the Chapter 3. The work reported in this chapter assumes the presence of collimating lenses at the transmitter and receiver that gives us a highly directional channel.

In section 4.1 we generalize the concept of frequency domain differential phase shift keying (FD-DPSK) modulation in which each pulse corresponds to many data symbols. Then we propose an adaptive algorithm to improve the bit error rate (BER). We also discuss the use of multiple transmitters and multiple receivers with FD-DPSK modulation to linearly increase throughput. In section 4.2 we employ amplitude shift keying (ASK) modulation where each pulse corresponds to only one data symbol. Finally, we present the achievable data rates using FDDQPSK and ASK modulation methods in different cities around US where the main variable parameters are humidity and temperature.

\section{FD-DPSK MODULATION}

We initially use frequency domain differential phase shift keying (FD-DPSK) modulation that is proposed by Rhee et al. [49]. In this method, the data is modulated based on the phase difference between adjacent spectral bands of the signal. The 
receiver retrieves the modulated data by means of wavelength shift with an interferogram.

For modulating the spectral domain of the pulsed signal, we assume an acoustooptic modulator (AOM) pulse shaper technique [50] [49]. In our system, the transmitter consists of a femtosecond pulse laser and a sequence of grating $\rightarrow$ lens $\rightarrow$ $\mathrm{AOM} \rightarrow$ lens $\rightarrow$ grating, as we saw in section 2.6.3.

The AOM of our system has a $40 \mathrm{~mm}$ aperture size with 1000 pixel spectral resolution. It takes $\sim 10 \mu \mathrm{s}$ for the input radio frequency $(\mathrm{RF})$, which has the carrier frequency of $200 \mathrm{MHz}$ and modulation bandwidth of $100 \mathrm{MHz}$, to propagate through the AOM. Meanwhile, the grating and lens slice the femtosecond pulse into 1000 bands. Since the bandwidth of our signal is $2 \mathrm{THz}$, each of these frequency bands is $2 \mathrm{GHz}$ wide. The sliced pulse is then passed through the AOM and phase modulated with the phase information of the modulating RF signal. As is clear from Fourier transform relations, this output pulse spreads in time and takes the time window equal to one of the spectral bands, which is 1 ns.

\subsubsection{FD-DMPSK Modulation}

We saw the general idea of FD-DBPSK modulation in 2.6.3. Here we generalize the modulation to FD-DMPSK modulation, where $M$ is the modulation order. Since we are using differential phase modulation methods, for sending $n$ symbols, $a_{i} \in\{1,2, \cdots, n\}$, the transmitter needs to send an extra symbol as the reference phase. Therefore, the spectrum of the pulse signal is sliced into $n+1$ bands.

The modulation process is as follows: consider $n$ symbols $a_{1}, a_{2}, a_{3}, \cdots, a_{n}$, where $a_{i} \in\{1,2, \cdots, M\}$ and $1,2, \cdots, M$ correspond to symbols having bit sequences such that the Hamming distance between each two adjacent symbols is

only 1 . That is every two consecutive symbols differ in only 1 bit of data, so that 


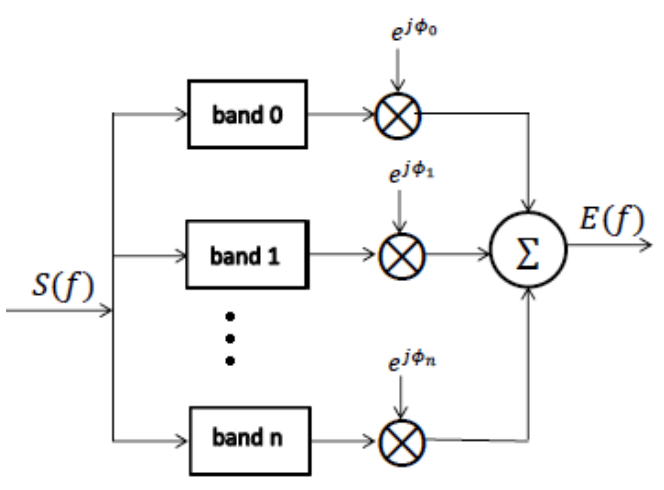

Figure 4.1: Transmitter block diagram.

we can minimize the number of detected errors in the receiver.

First, the phase function, $\psi\left(a_{i}\right)$, for each of these symbols is set as follows:

$$
\begin{array}{r}
\psi\left(a_{i}\right)=\frac{\left(a_{i}-1\right) \pi}{2^{b}-1}, \\
\text { for } i=1, \cdots, n
\end{array}
$$

Then, the corresponding $n+1$ phases are found:

$$
\phi_{i}= \begin{cases}0 & \text { for } i=0, \\ \psi\left(a_{i}\right)+\phi_{i-1}, & \text { for } i=1,2, \cdots, n\end{cases}
$$

where $\phi_{0}$ corresponds to the reference phase.

Figure 4.1 shows the transmitter block diagram. We generate the femtosecond pulse as a step rectangular pulse function that corresponds to a sinc function with bandwidth of $2 \mathrm{THz}$ in the frequency domain. We slice the frequency domain of the signal into $n+1$ bands and then each band is modulated with the phase obtained from (4.2) as below.

$$
E(f)=\sum_{i=0}^{n} \mathrm{e}^{j \phi_{i}} S\left(f-f_{i}, \delta f\right)
$$




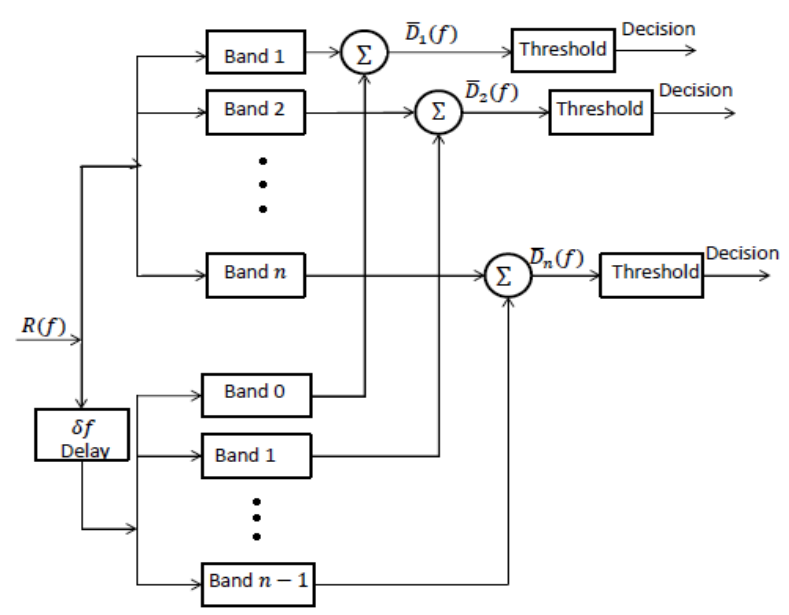

Figure 4.2: Receiver block diagram.

Here, $E(f)$ is the spectral complex value of the femtosecond signal and $S\left(f-f_{i}, \delta f\right)$ is the spectral value of $i$ th band. Note that $f_{i}=f_{0}+i \delta f$ is the central frequency, and $\delta f=\Delta f /(n+1)$ is the bandwidth of the $i$ th band. Here, $f_{0}$ is the central frequency of the first spectral band and $\Delta f=2 \mathrm{THz}$. The modulated signal is then passed through the channel and is changed based on the channel model.

$$
R(f)=E(f) H(f)+\text { Noise }
$$

where, $H(f)$ is the transfer function of the directional channel from (3.17), Noise is the noise in the channel that is added to the signal, and $R(f)$ is the received signal at the receiver.

At the receiver, the signal is first divided by the transfer function of the channel, $H(f)$, to decrease the effect of channel impairments. Figure 4.2 shows the receiver block diagram. As can be seen from the figure, the received signal, $R(f)$, is first passed through a filter and is sliced into $n+1$ bands. A copy of the signal is shifted in frequency by $\delta f$. Thus, the shifted $(i-1)$ th band has the central frequency of the unshifted $i$ th band. Combining the $i$ th band of the unshifted signal with the 
$(i-1)$ th band in the shifted signal we get the following.

$$
\begin{aligned}
& \bar{D}_{i}(f)=\frac{1}{2}\left[R\left(f-f_{i-1}, \delta f\right)+R\left(f-f_{i}, \delta f\right)\right], \\
& \quad \text { for } i=1, \cdots, n
\end{aligned}
$$

In (4.5), $R\left(f-f_{i}, \delta f\right)$ is the $i$ th band of the received signal, and $R\left(f-f_{i}, \delta f\right)$ is the $i$ th band of the received signal that is shifted by $\delta f$.

We now compare the intensity of the $\bar{D}_{i}(f),\left|\bar{D}_{i}(f)\right|^{2}$, with different thresholds at the receiver that are found based on the following equations.

$$
\begin{gathered}
\bar{E}_{i}(f)=\frac{1}{2}\left(\mathrm{e}^{j \phi_{i-1}} S\left(f-f_{i-1}, \delta f\right)+\right. \\
\left.\qquad \mathrm{e}^{j \phi_{i}} S\left(f-f_{i}, \delta f\right)\right) \\
\text { for } i=1, \cdots, n \\
\left|\bar{E}_{i}(f)\right|^{2}=\frac{1}{2}\left|\bar{S}_{i}(f)\right|^{2} \times\left|1+\mathrm{e}^{2 j \psi\left(a_{i}\right)}+2 \mathrm{e}^{j \psi\left(a_{i}\right)}\right| \\
\text { for } i=1, \cdots, n
\end{gathered}
$$

Here, $\bar{S}_{i}(f)$ is the average of each two consecutive unmodulated spectral bands and is found as follows.

$$
\begin{aligned}
& \bar{S}_{i}(f)=\frac{1}{2}\left[S\left(f-f_{i-1}, \delta f\right)+S\left(f-f_{i}, \delta f\right)\right], \\
& \text { for } i=1, \cdots, n
\end{aligned}
$$

For $n$ symbols we get $(n-1)$ thresholds.

$$
\begin{gathered}
t h_{i}^{j-1}=\frac{\left|\bar{E}_{i}(f)\right|_{a_{j-1}}^{2}+\left|\bar{E}_{i}(f)\right|_{a_{j}}^{2}}{2}, \\
\text { for } j=2, \cdots, n
\end{gathered}
$$




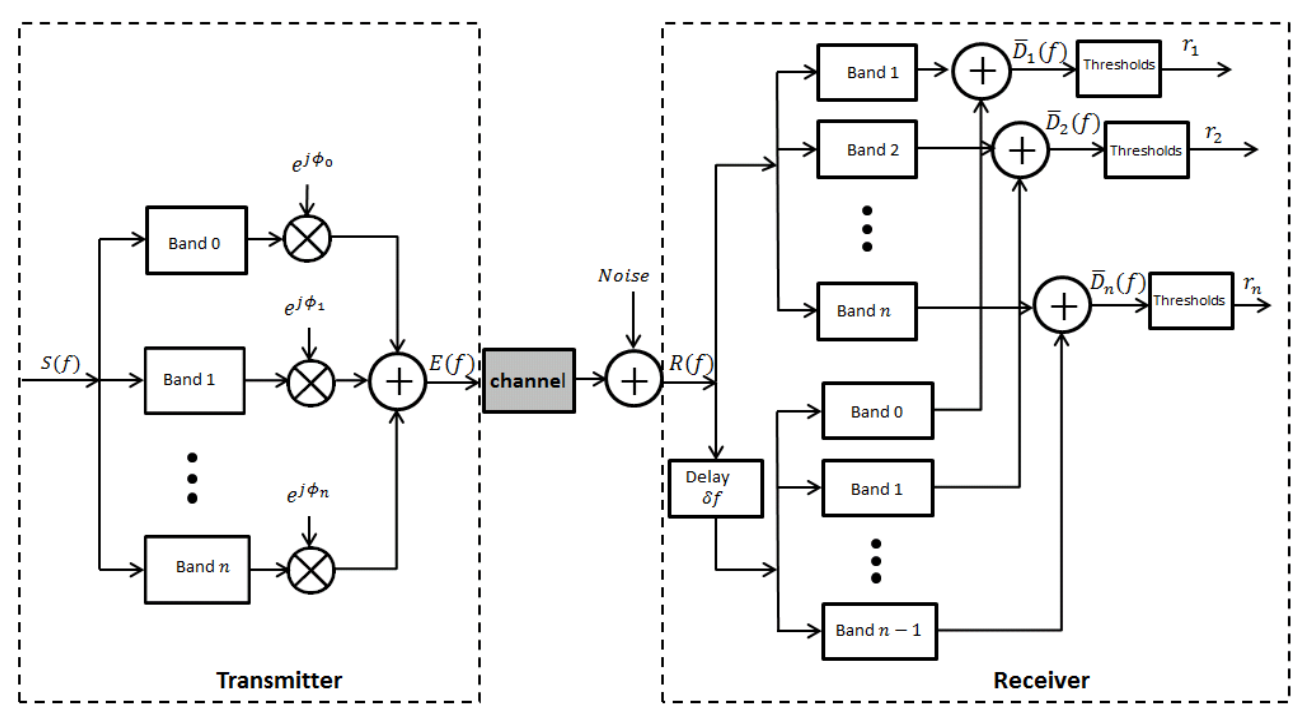

Figure 4.3: transmitter, receiver, and channel block diagram for FD-DmPSK, where $m$ is $2,4,8,16$, and 64 .

Having the thresholds, the received symbols are detected. Where, $r_{i}$ is the $i$ th detected symbol in the receiver.

$$
r_{i}= \begin{cases}a_{1}, & \text { if }\left|\bar{D}_{i}(f)\right|^{2} \geq t h_{i}^{1} \\ a_{2}, & \text { else if }\left|\bar{D}_{i}(f)\right|^{2} \geq t h_{i}^{2} \\ \vdots & \\ a_{n-1}, & \text { else if }\left|\bar{D}_{i}(f)\right|^{2} \geq t h_{i}^{n-1} \\ a_{n}, & \text { otherwise }\end{cases}
$$

Figure 4.3 is the block diagram of the system that shows transmitter, receiver and the channel together. 
Table 4.1: The list of figures that show BER vs $\mathrm{E}_{\mathrm{b}} / \mathrm{N}_{0}$ for different modulation schemes corresponding to transmitter-receiver (Tx-Rx) distances of $50 \mathrm{~cm}$ and $100 \mathrm{~cm}$.

\begin{tabular}{|c|ccc|}
\hline \multirow{2}{*}{ Tx-Rx Distance } & \multicolumn{3}{|c|}{$\mathbf{R H}$} \\
\cline { 2 - 4 } & $\mathbf{0 \%}$ & $\mathbf{4 0 \%}$ & $\mathbf{8 0 \%}$ \\
\hline $\mathbf{5 0} \mathbf{~ c m}$ & Figure 4.4 & Figure 4.5 & Figure 4.6 \\
$\mathbf{1 0 0} \mathbf{~ c m ~}$ & Figure 4.7 & Figure 4.8 & Figure 4.9 \\
\hline
\end{tabular}

\subsubsection{Simulation Results for FD-DMPSK}

We use Matlab to simulate the communication system in Figure 4.3. We provide the results of our simulation for each modulation scheme in the following subsections. The code is provided in Appendix D.

We utilize 5 different modulation schemes: FD-DBPSK, FD-DQPSK, FDD8PSK, FD-D16PSK, and FD-D64PSK. For each of these modulations, we consider $50 \mathrm{~cm}$ and $100 \mathrm{~cm}$ distances between transmitter and receiver. Each case is simulated for different RH levels of $0 \%, 40 \%$, and $80 \%$.

Table 4.1 lists the figures that show BER vs $\mathrm{E}_{\mathrm{b}} / \mathrm{N}_{0}$ for different modulation schemes.

In these figures, the dashed line with square corresponds to FD-DBPSK, dashed line corresponds to FD-DQPSK, solid line with triangle corresponds to FD-D8PSK, solid line with circle corresponds to D16PSK, and solid line corresponds to D64PSK. We ignore the cases with $\mathrm{BER}<10^{-6}$. In Figures 4.4 and 4.7, when the relative humidity is $0 \%$ and $\mathrm{E}_{\mathrm{b}} / \mathrm{N}_{0}>-80 \mathrm{~dB}$, the BER corresponding to FD-DBPSK, FD-DQPSK, and FD-D8PSK, is equal to 0.

It is interesting to note that the BER curves for all modulation schemes are 


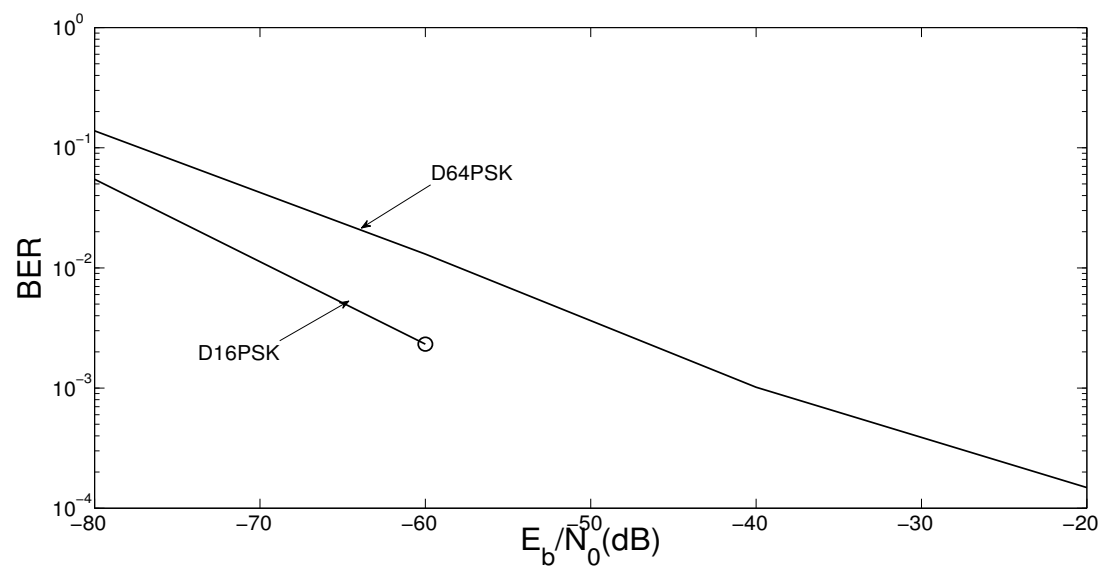

Figure 4.4: BER vs $\mathrm{E}_{\mathrm{b}} / \mathrm{N}_{0}$ for $0 \% \mathrm{RH}$ and transmitter-receiver distance of $50 \mathrm{~cm}$.

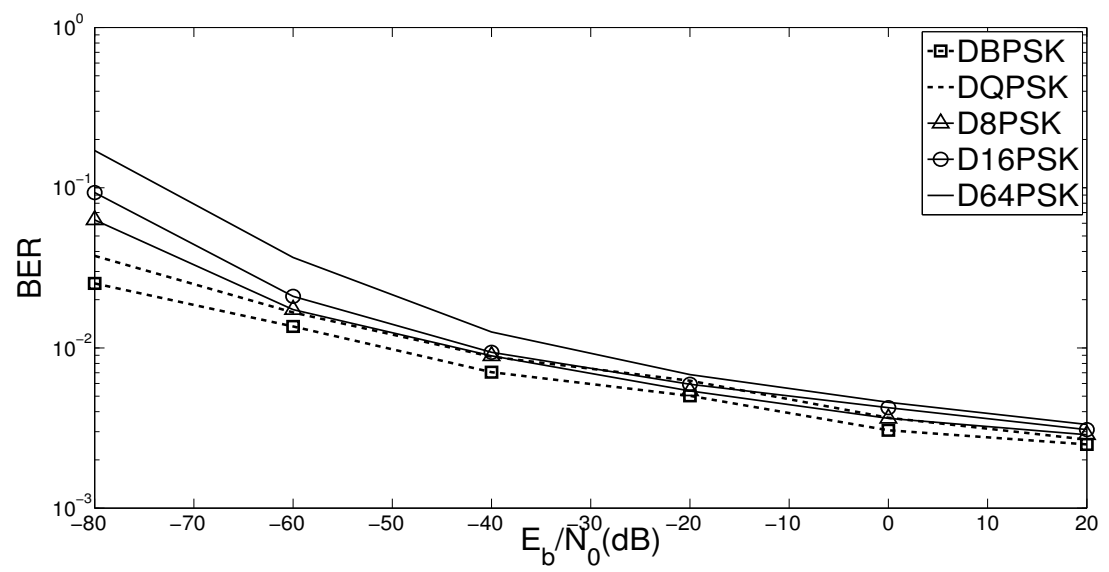

Figure 4.5: BER vs $\mathrm{E}_{\mathrm{b}} / \mathrm{N}_{0}$ for $40 \% \mathrm{RH}$ and transmitter-receiver distance of $50 \mathrm{~cm}$.

close to one another. The reason is that for higher order modulation schemes we need to increase the transmitted signal power to maintain the same $E_{b} / N_{0}$. Therefore, BER is not degraded drastically as we use a higher order modulation method. 


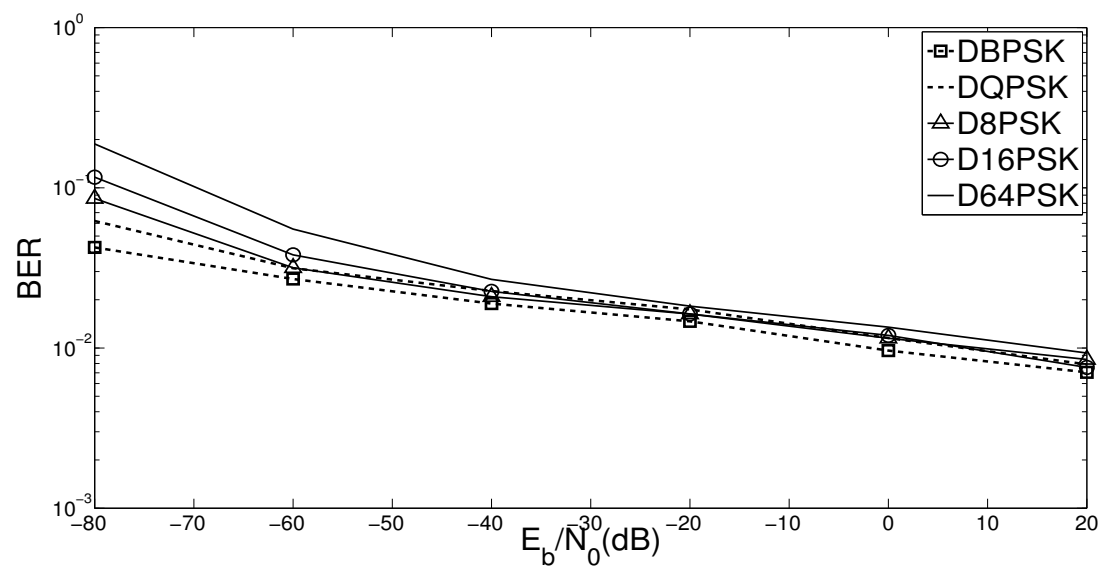

Figure 4.6: BER vs $\mathrm{E}_{\mathrm{b}} / \mathrm{N}_{0}$ for $80 \% \mathrm{RH}$ and transmitter-receiver distance of $50 \mathrm{~cm}$.

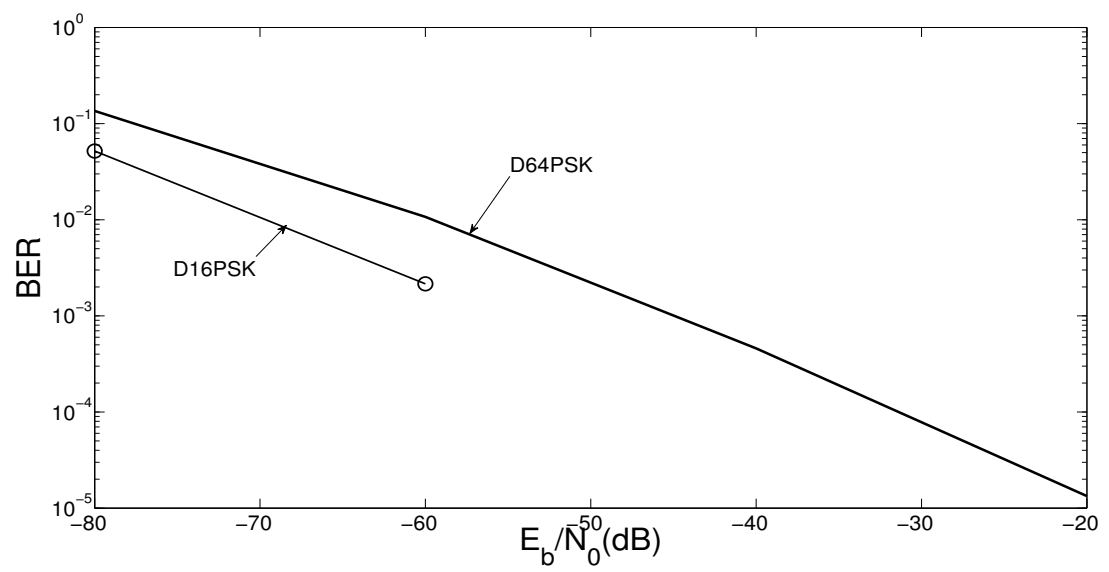

Figure 4.7: BER vs $\mathrm{E}_{\mathrm{b}} / \mathrm{N}_{0}$ for $0 \% \mathrm{RH}$ and transmitter-receiver distance of $100 \mathrm{~cm}$.

\subsubsection{Adaptive FD-DPSK Modulation}

As atmospheric attenuation depends on frequency, some bands are attenuated drastically. We exclude these bands from the modulation. The set of bands that are excluded depends on the transmitter-receiver distance as well as the humidity level. Thus, at lower humidity or smaller distances, fewer bands are excluded, since the attenuation is not significant. While this adaptive method decreases the number of symbols that are sent, it enhances the BER for the same energy per bit 


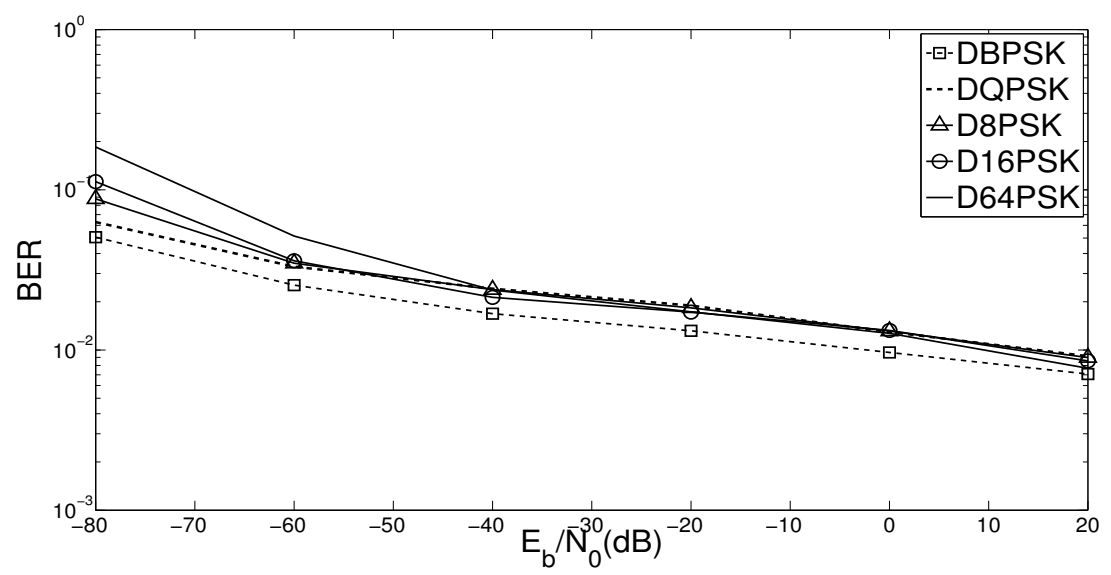

Figure 4.8: BER vs $\mathrm{E}_{\mathrm{b}} / \mathrm{N}_{0}$ for $40 \% \mathrm{RH}$ and transmitter-receiver distance of $100 \mathrm{~cm}$.

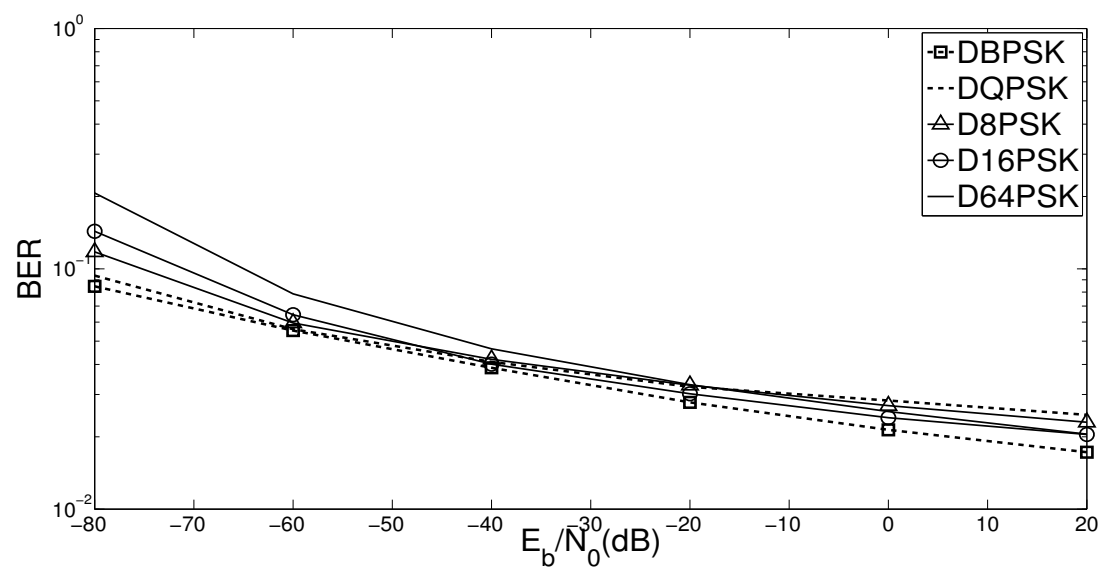

Figure 4.9: BER vs $\mathrm{E}_{\mathrm{b}} / \mathrm{N}_{0}$ for $80 \% \mathrm{RH}$ and transmitter-receiver distance of $100 \mathrm{~cm}$.

to the spectral noise density $\left(\mathrm{E}_{\mathrm{b}} / \mathrm{N}_{0}\right)$. Figure 4.10 shows the error rate for each band and the corresponding humidity absorption coefficient when the $E_{b} / N_{0}=0$ $\mathrm{dB}$, transmitter-receiver distance is $100 \mathrm{~cm}$, and the relative humidity is $80 \%$. As is shown in the figure, all of the bands that cause errors have center frequency higher than $1 \mathrm{THz}$. The reason is that the sync signal in our simulation has more power for frequencies below $1 \mathrm{THz}$ and even though it is attenuated, the corresponding bands are detectable at the receiver. On the other hand, the sync signal has less 


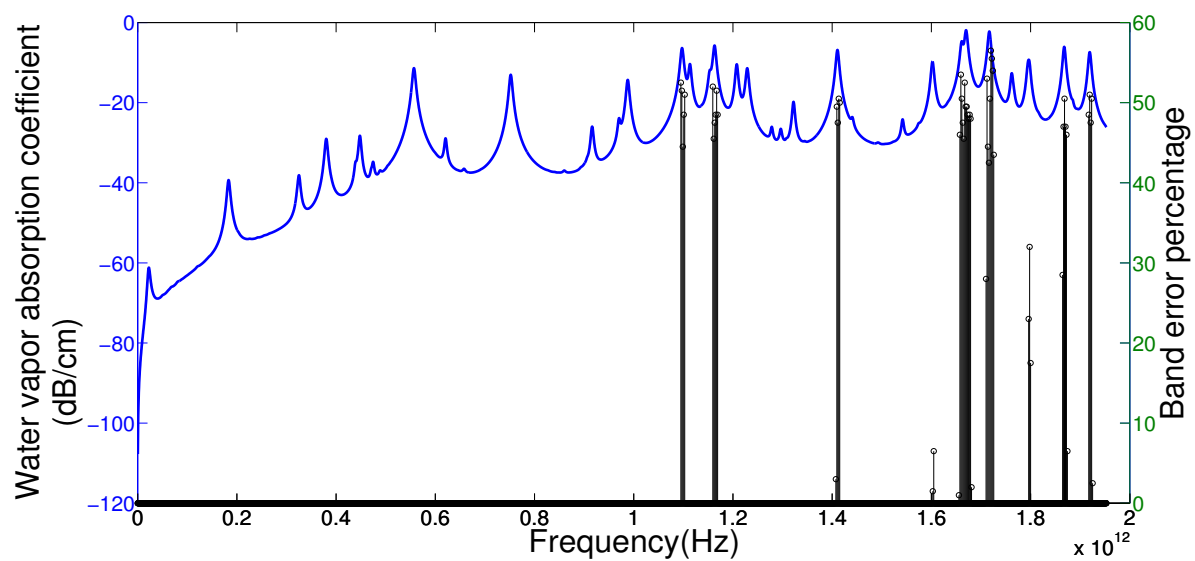

Figure 4.10: Bands' error rate vs water vapor absorption coefficient for $\mathrm{E}_{\mathrm{b}} / \mathrm{N}_{0}=0 \mathrm{~dB}$, transmitter-receiver distance $=100 \mathrm{~cm}$, and $\mathrm{RH}=80 \%$

power for the bands with center frequency of higher than $1 \mathrm{THz}$. Hence, the attenuation will cause errors in these bands.

We exclude the erroneous bands from the modulation based on the following algorithm:

- The available bands are categorized into two groups:

1. error-free bands: bands that will carry useful information,

2. erroneous bands: bands that should be excluded from the modulation,

- At the transmitter

1. The phase corresponding to error-free bands are found from (4.2),

2. The phase corresponding to erroneous bands are set to 0,

3. RF signal, which is the superposition of phases found in steps 1 and 2 , is used to modulate the pulse.

- Knowing the erroneous bands, the receiver will ignore them and use the remaining bands to decode data. 
Table 4.2: Number of erroneous bands with $\mathrm{E}_{\mathrm{b}} / \mathrm{N}_{0}=0$ and BER $\geq 10^{-2}$ for FD-DPSK scheme and transmitter-receiver (Tx-Rx) distances of $50 \mathrm{~cm}$ and $100 \mathrm{~cm}$.

\begin{tabular}{|c|cc|}
\hline \multirow{2}{*}{ Tx-Rx Distance } & \multicolumn{2}{|c|}{$\mathbf{R H}$} \\
\cline { 2 - 3 } & $\mathbf{4 0 \%}$ & $\mathbf{8 0 \%}$ \\
\hline $\mathbf{5 0} \mathbf{~ c m}$ & 9 & 29 \\
$\mathbf{1 0 0} \mathbf{~ c m}$ & 30 & 55 \\
\hline
\end{tabular}

Using the adaptive algorithm, we find the erroneous bands and exclude them from our modulation.

\subsubsection{Simulation Results for Adaptive FD-MDPSK Modulation}

In adaptive FD-DPSK, we find the erroneous bands for $\mathrm{E}_{\mathrm{b}} / \mathrm{N}_{0}=0$ that have $\mathrm{BER} \geq 10^{-2}$. Table 4.2 shows the number of erroneous bands for different scenarios.

Figures 4.11 and 4.12 compare BER vs $\mathrm{E}_{\mathrm{b}} / \mathrm{N}_{0}$ of adaptive modulation schemes when transmitter-receiver distance is $50 \mathrm{~cm}$ and the $\mathrm{RH}$ is $40 \%$ and $80 \%$, respectively.

Figures 4.13 and 4.14 compare BER vs $\mathrm{E}_{\mathrm{b}} / \mathrm{N}_{0}$ of modulation schemes when transmitter-receiver distance is $100 \mathrm{~cm}$ and $\mathrm{RH}$ is $40 \%$ and $80 \%$, respectively. In these figures the dashed line with square corresponds to FD-DBPSK, dashed line corresponds to FD-DQPSK, solid line with triangle corresponds to FD-D8PSK, solid line with circle corresponds to D16PSK, and solid line corresponds to D64PSK.

As is obvious from the figures, the BER has decreased significantly using adaptive modulation schemes in comparison with the regular modulation schemes. For example, at $\mathrm{E}_{\mathrm{b}} / \mathrm{N}_{0}=20 \mathrm{~dB}$, with $50 \mathrm{~cm}$ transmitter-receiver distance and $80 \%$ $\mathrm{RH}, \mathrm{BER} \sim 10^{-2}$ has been reduced to $\sim 10^{-4}$ in FD-D64PSK, to $\sim 10^{-5}$ in 


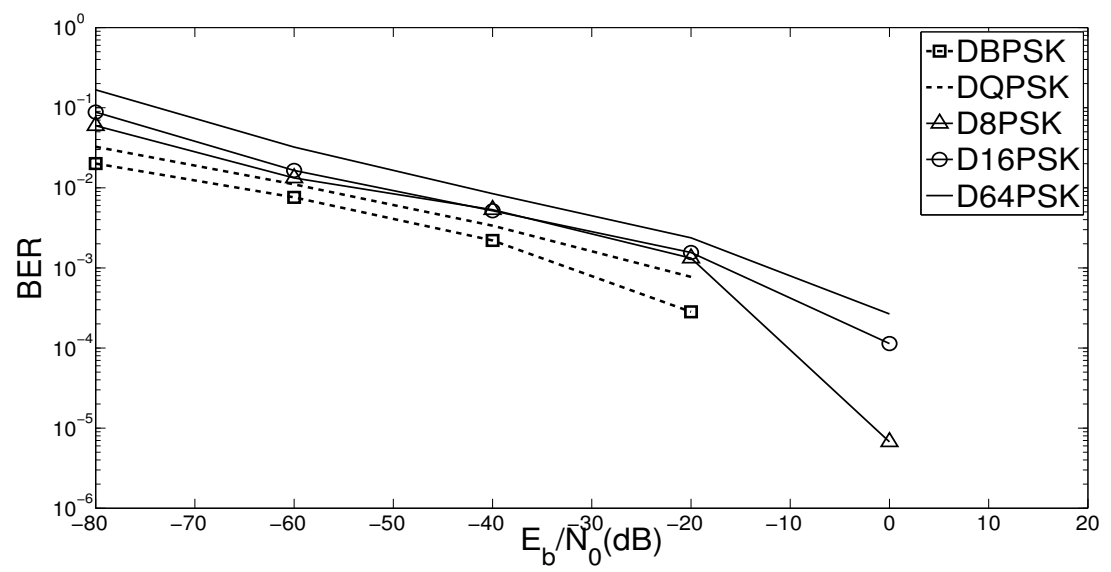

Figure 4.11: BER vs $\mathrm{E}_{\mathrm{b}} / \mathrm{N}_{0}$ corresponding adaptive modulation schemes for $40 \% \mathrm{RH}$ and transmitter-receiver distance of $50 \mathrm{~cm}$.

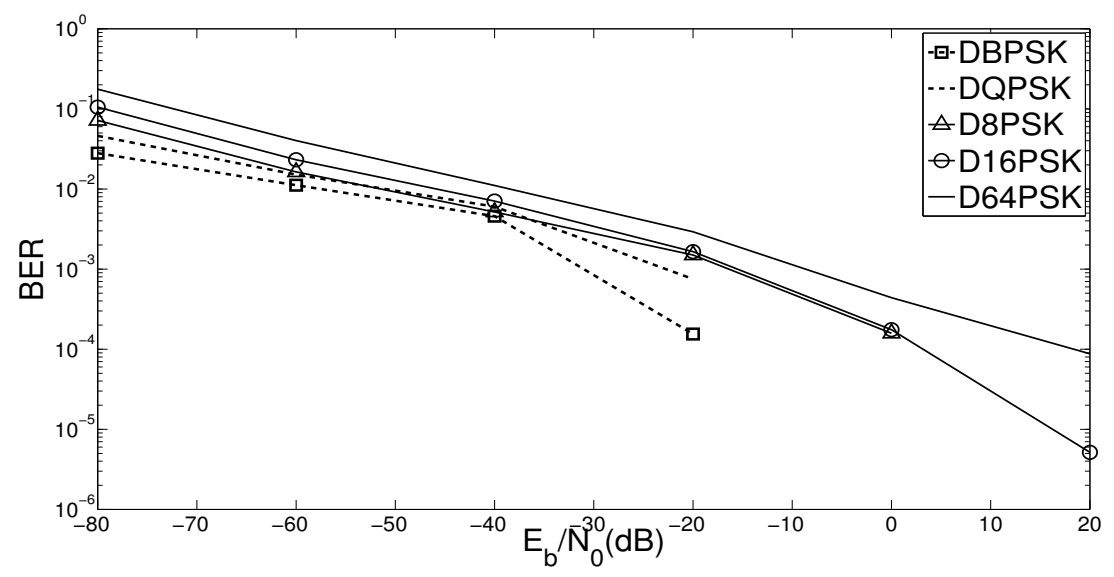

Figure 4.12: $\mathrm{BER}$ vs $\mathrm{E}_{\mathrm{b}} / \mathrm{N}_{0}$ corresponding adaptive modulation schemes for $80 \% \mathrm{RH}$ and transmitter-receiver distance of $50 \mathrm{~cm}$.

FD-D16PSK, and 0 for FD-D8PSK, FD-DQPSK, and FD-DBPSK. 


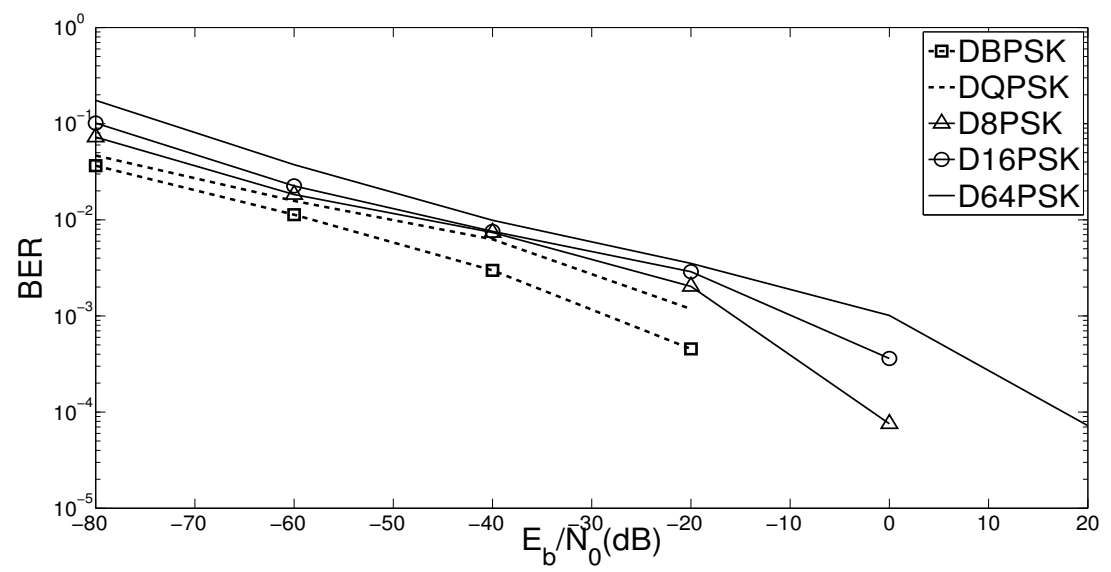

Figure 4.13: BER vs $\mathrm{E}_{\mathrm{b}} / \mathrm{N}_{0}$ corresponding adaptive modulation schemes for $40 \% \mathrm{RH}$ and transmitter-receiver distance of $100 \mathrm{~cm}$.

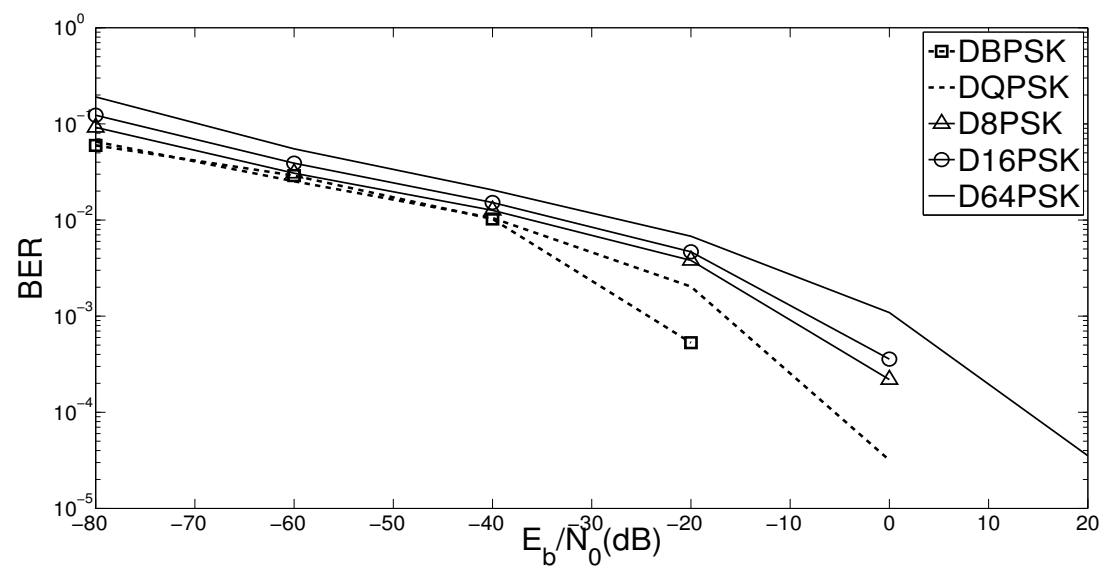

Figure 4.14: $\mathrm{BER}$ vs $\mathrm{E}_{\mathrm{b}} / \mathrm{N}_{0}$ corresponding adaptive modulation schemes for $80 \% \mathrm{RH}$ and transmitter-receiver distance of $100 \mathrm{~cm}$.

\subsubsection{Multiple Transmitters and Achievable Data Rates Based on Adap- tive FD-DPSK Modulation}

As explained earlier in section 4.1, based on the AOM structure and its aperture size, the transmitter sends a pulse every $10 \mu$ s that takes 1 ns. Therefore, up to 10000 transmitters can be incorporated in a time division multiplexing (TDM) 


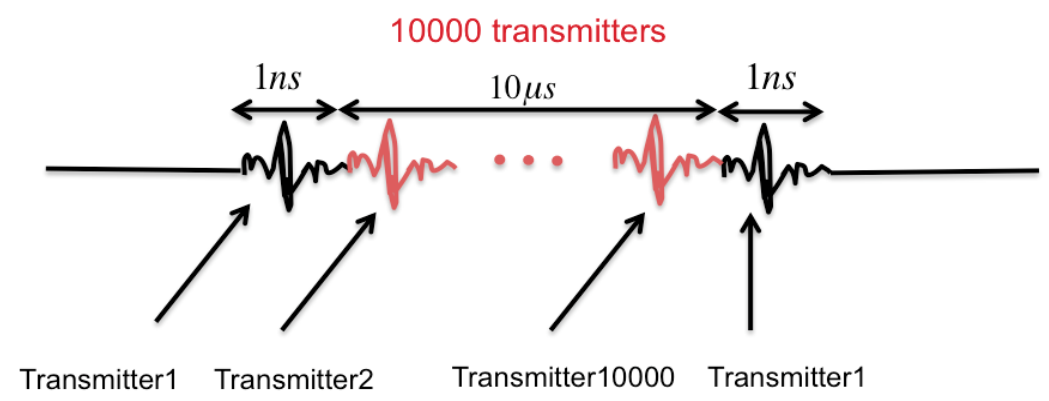

Figure 4.15: Up to 10'000 transmitters can generate pulses in a TDM system and achieve higher data rates.

system to achieve higher data rates. Figure 4.15 shows how this idea works. Having multiple transmitters, Table 4.3 shows the aggregate number of error-free detected bits at the receiver, when $\mathrm{E}_{\mathrm{b}} / \mathrm{N}_{0}=0(\mathrm{~dB})$.

\subsubsection{Achievable Data Rates in Directional THz Channels}

It is been only few years since the development of hardware that generates and detects $\mathrm{THz}$ pulses. Therefore, the technology is new and it is improving every year. One of the limitations with current hardware is the pulse generation and detection speed. For example, the Pricometrix T-ray 4000 system that we use in our lab experiments generates 100 million pulses per second that is $10^{8}=0.1 \mathrm{GHz}$ [56]. For our simulations we assume a relaxation time of 1 ns. That is the time between the end and the start of two consecutive pulses.

We simulate pulses such that the corresponding bandwidth in frequency domain is $2 \mathrm{THz}$. Where the relation between the pulse length, $T$, in time domain and the corresponding pulse bandwidth, $B$, is $T=\frac{1}{B}$. Therefore, the pulse length $T$ is equal to $5 \times 10^{-13}=0.5 \times 10^{-12}$ s or $0.5 \mathrm{ps}$.

Based on the above assumptions, we calculate the upper bound of data rate as 
Table 4.3: Adaptive FD-DQPSK bit rate for multiple scenarios with $\mathrm{E}_{\mathrm{b}} / \mathrm{N}_{0}=0 \mathrm{~dB}$

\begin{tabular}{|c|c|c|c|}
\hline & \multirow[b]{2}{*}{ RH } & \multicolumn{2}{|c|}{ Distance } \\
\hline & & $50(\mathrm{~cm})$ & $100(\mathrm{~cm})$ \\
\hline \multirow{3}{*}{ DBPSK } & $0 \%$ & $0.999 \mathrm{Tbit} / \mathrm{s}$ & $0.999 \mathrm{Tbit} / \mathrm{s}$ \\
\hline & $40 \%$ & $0.99 \mathrm{Tbit} / \mathrm{s}$ & $0.969 \mathrm{Tbit} / \mathrm{s}$ \\
\hline & $80 \%$ & $0.97 \mathrm{Tbit} / \mathrm{s}$ & $0.944 \mathrm{Tbit} / \mathrm{s}$ \\
\hline \multirow{3}{*}{ DQPSK } & $0 \%$ & $1.998 \mathrm{Tbit} / \mathrm{s}$ & $1.998 \mathrm{Tbit} / \mathrm{s}$ \\
\hline & $40 \%$ & $1.98 \mathrm{Tbit} / \mathrm{s}$ & $1.938 \mathrm{Tbit} / \mathrm{s}$ \\
\hline & $80 \%$ & $1.94 \mathrm{Tbit} / \mathrm{s}$ & $1.887 \mathrm{Tbit} / \mathrm{s}$ \\
\hline \multirow{3}{*}{ D8PSK } & $0 \%$ & 2.997 Tbit/s & $2.997 \mathrm{Tbit} / \mathrm{s}$ \\
\hline & $40 \%$ & $2.97 \mathrm{Tbit} / \mathrm{s}$ & $2.906 \mathrm{Tbit} / \mathrm{s}$ \\
\hline & $80 \%$ & $2.9095 \mathrm{Tbit} / \mathrm{s}$ & $2.8314 \mathrm{Tbit} / \mathrm{s}$ \\
\hline \multirow{3}{*}{ D16PSK } & $0 \%$ & $3.996 \mathrm{Tbit} / \mathrm{s}$ & $3.996 \mathrm{Tbit} / \mathrm{s}$ \\
\hline & $40 \%$ & $3.9595 \mathrm{Tbit} / \mathrm{s}$ & $3.8746 \mathrm{Tbit} / \mathrm{s}$ \\
\hline & $80 \%$ & $3.8793 \mathrm{Tbit} / \mathrm{s}$ & $3.7746 \mathrm{Tbit} / \mathrm{s}$ \\
\hline \multirow{3}{*}{ D64PSK } & $0 \%$ & $5.994 \mathrm{Tbit} / \mathrm{s}$ & $5.994 \mathrm{Tbit} / \mathrm{s}$ \\
\hline & $40 \%$ & $5.938 \mathrm{Tbit} / \mathrm{s}$ & $5.8081 \mathrm{Tbit} / \mathrm{s}$ \\
\hline & $80 \%$ & 5.817 Tbit/s & $5.657 \mathrm{Tbit} / \mathrm{s}$ \\
\hline
\end{tabular}

follows.

$$
b=b_{1}-\left(\mathrm{BER} \times b_{1}\right)
$$

Here, $b$ is bit rate, $b_{1}$ is initial bit rate that is the bit rate when BER is equal to 0 and is found from the following equation.

$$
b_{1}=\frac{s_{l}}{T+T_{p}}
$$

Where, $s_{l}$ is the number of bits per pulse, $T$ is the pulse length that is equal to 0.5 
Table 4.4: Relative Humidity $(\mathrm{RH})$ for cities in summer and winter

\begin{tabular}{ccc}
\hline \hline City & Summer RH & Winter RH \\
\hline Las Vegas (NV) & $21 \%$ & $42 \%$ \\
Sacramento (CA) & $54 \%$ & $76 \%$ \\
Omaha (NE) & $60 \%$ & $64 \%$ \\
New York (NY) & $65 \%$ & $63 \%$ \\
Portland (OR) & $64 \%$ & $79 \%$ \\
Washington (D.C.) & $68 \%$ & $66 \%$ \\
Miami (FL) & $73 \%$ & $70 \%$ \\
Fargo (ND) & $73 \%$ & $76 \%$ \\
Chicago (IL) & $72 \%$ & $74 \%$ \\
Austin (TX) & $73 \%$ & $71 \%$ \\
Los Angeles (CA) & $77 \%$ & $66 \%$ \\
\hline
\end{tabular}

$\mathrm{ps}$, and $T_{p}$ is the relaxation time that is equal to $1 \mathrm{~ns}$.

\subsubsection{Data Rate for Cities Based on Adaptive FD-DQPSK}

To estimate the achievable data rate in different cities in U.S. using adaptive modulation scheme, we first calculate the average relative humidity for cities around U.S. for summer and winter from [3]. Table 4.4 shows these cities with their relative humidity in summer and winter. Then we simulate the adaptive FD-DQPSK modulation to find the average data rate vs distance for these cities. Figure 4.16 shows the result of the simulation. As the figure shows, data rate is decreased when the distance and/or humidity is increased. That is as humidity and/or distance is increased, the adaptive FD-DQPSK deletes more bands from the modulation to enhance the BER. 


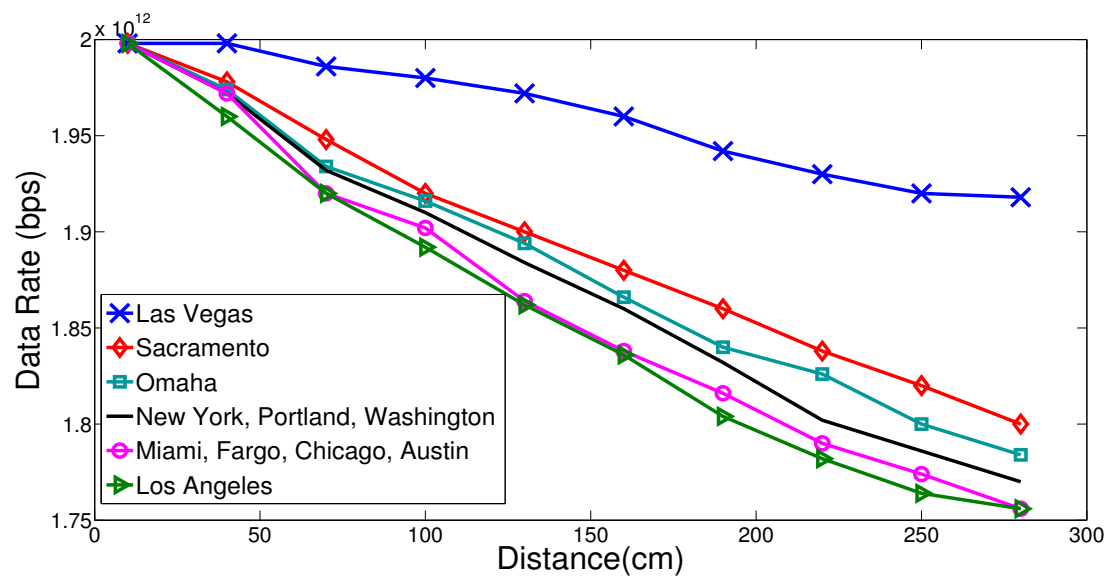

(a)

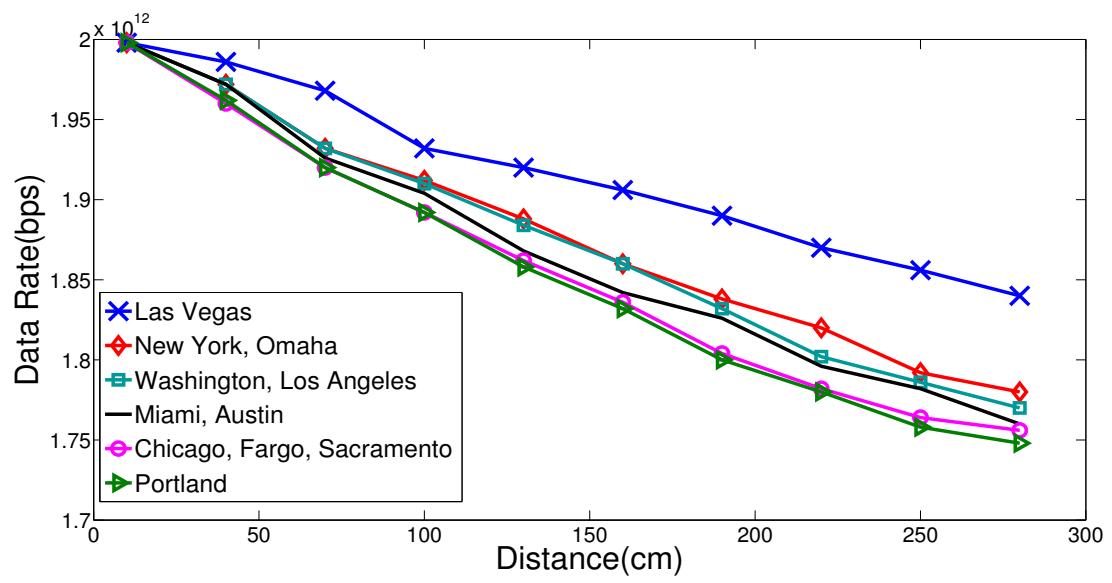

(b)

Figure 4.16: Adaptive FD-DQPSK modulation data rate vs distance $(\mathrm{cm})$ for cities in (a) summer and (b) winter.

\subsubsection{Multiple Users in FD-DPSK Modulation}

When we use the FD-DQPSK scheme, since we are splitting the $2 \mathrm{THz}$ bandwidth into $2 \mathrm{GHz}$ bands, the reconstituted pulse spreads in time and is now $1 \mathrm{~ns}$ long. Note that the AOM takes $10 \mu$ s to set to the next symbol. Therefore, by using 10,000 AOMs staggered in time with respect to each other by 1ns, we can fully 


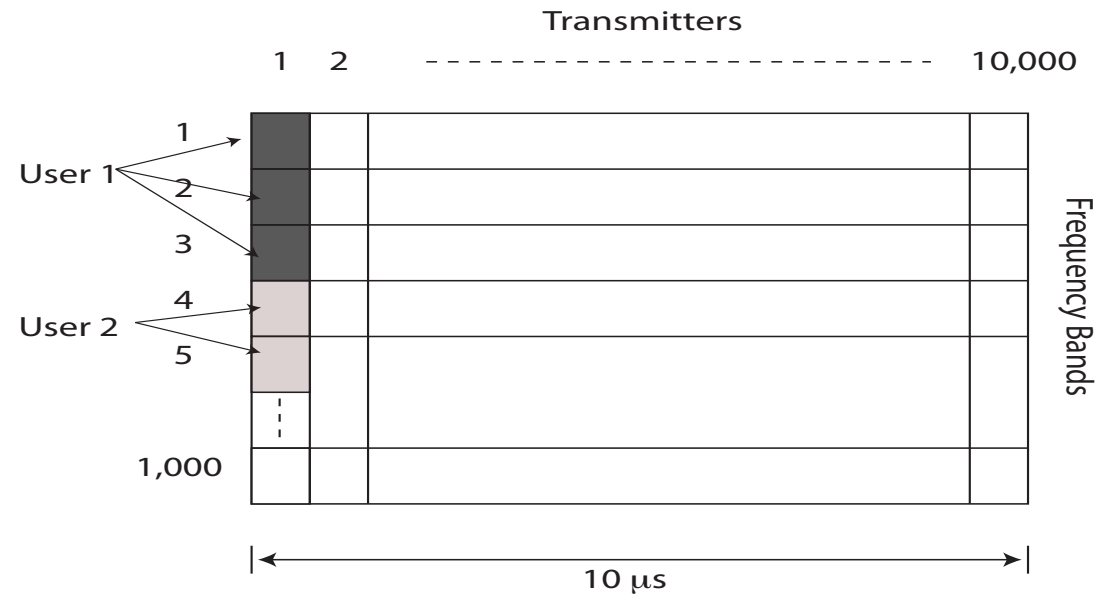

Figure 4.17: Using multiple transmitters.

occupy the channel as shown in Figure 4.17. In the figure we illustrate the 1000, 2 $\mathrm{GHz}$ channels and consecutive AOMs used to generate the modulated pulse. Using this simple scheme, we can achieve aggregate data rates of $1.98 \mathrm{Tbit} / \mathrm{s}$ and 1.94 Tbit/s when the transmitter-receiver distance is $50 \mathrm{~cm}$ and the relative humidity is $40 \%$ and $80 \%$, respectively. The figure also illustrates how we can use this channel structure to support multiple simultaneous users. In the figure we show that user 1 is allocated 3 slots, where a slot is a combination of frequency band and AOM. If we assume that user 1 only gets 3 slots every $10 \mu$ s the data rate it can achieve is $400 \mathrm{kbps}$. This is because one of the slots serves as the reference phase while the other two are each carrying 2 bits of data. It is easy to see that this structure will allow an access point to multiplex data streams for many users in a very flexible way.

Finally, we need to discuss the question of how a receiver can determine its slot allocation as well as which of the bands is not being used (due to the weakness of the signal caused by humidity and distance). A possible solution is to use designated lower frequency bands (below $300 \mathrm{GHz}$ ) for sending control information at some periodicity since these bands are relatively immune to water absorption. 
Therefore, in our proposed approach, the transmitter first estimates the relative humidity and uses some form of ranging to determine the distance at which each receiver is located. It then estimates which bands should not be used for each user, performs an allocation of slots and transmits this information in the control slots. Interestingly, some slots may be usable for nearby users and not for distant users. This gives us more choices in determining slot allocation.

\section{ASK MODULATION METHODS}

Now we discuss simpler modulation methods that only change the pulse amplitude to encode information on the transmitted signal. Here, each pulse corresponds to only one symbol. The reason to consider these modulation methods is the ease of implementation as compared with FD-DPSK.

\subsubsection{ASK Modulation}

In on-off keying (OOK) modulation each symbol corresponds to 1 bit of data. As we saw in section 2.6 .2 , by changing the amplitude of the pulse we can create two different states to send and detect a " 0 " and a "1". However, we differentiate between the cases where a " 0 " is sent and where "No-data" is sent in our analysis. That is, we use three different states to represent " 1 ", " 0 ", and the case that "No-data" has been sent and we call it 3ASK modulation. Lets assume that amplitude $a$ corresponds to the pulse that represents a " 1 ", pulse with amplitude $b$ corresponds to a " 0 ", and pulse with amplitude 0 (no pulse) corresponds to the case where "No-data" has been sent. To better differentiate between the pulse with amplitude $a$ and the pulse with amplitude $b$, we chose $b$ such that it is $a / 2$. Note that the power of a pulse in ASK modulation with amplitude $a$ is equal to 
$a^{2}$ as the following equation shows.

$$
P=\frac{1}{T} \int_{0}^{T} x(t)^{2} d t=\frac{1}{T} \int_{0}^{T} a^{2} d t=a^{2}
$$

Therefore, if the probability of having pulses with amplitudes $a, b$, and $c$ is the same and is equal to $1 / 3$, we can write the following relation.

$$
\begin{aligned}
& \mathrm{E}[\text { power }]=\frac{1}{3} \times a^{2}+\frac{1}{3} \times b^{2}+\frac{1}{3} \times 0=P, \\
& b=\frac{a}{2}
\end{aligned}
$$

where, $P$ is the signal power and $\mathrm{E}[$ power] is the expectancy of the signal power. Therefore, we can find $a$ and $b$ as follows.

$$
\begin{aligned}
& a=2 \sqrt{\frac{3}{5} \times P} \\
& b=\sqrt{\frac{3}{5} \times P}
\end{aligned}
$$

\section{Non-Coherent Detection of 3ASK Modulated Signal}

We calculate Fourier transform of the received signal $r(t)$ and we call it $R(f)$. Then we divide $R(f)$ by the transfer function of the channel $H(f)$ to minimize the effect of channel impairments. This new signal in time domain is called $r(t)$.

In ASK modulation, the information is conveyed in the amplitude or envelope of the modulated pulse signal and thus the data can be recovered using an envelope detector. For that, a matched filter is used to detect the received signal. That is, the receiver calculates the correlation of the received signal and the prototype signal that is available for each case. Lets assume that the prototype signal corresponding to data bit " 1 " is $s_{1}$, the prototype signal corresponding to data bit 0 is $s_{2}$, and the prototype signal corresponding to No-data is $s_{3}$. The receiver then compares the result with some thresholds that are defined as follows.

$$
T h_{1}=\left(\sum_{n=0}^{T-1} s_{1}^{2}+\sum_{n=0}^{T-1} s_{2}^{2}\right) / 2
$$




$$
T h_{2}=\left(\sum_{n=0}^{T-1} s_{2}^{2}+\sum_{n=0}^{T-1} s_{3}^{2}\right) / 2
$$

Where, $T$ is symbol duration in seconds, $\sum_{n=0}^{T-1} s_{1}^{2}$ is auto-correlation of $s_{1}, \sum_{n=0}^{T-1} s_{2}^{2}$ is auto-correlation of $s_{2}$, and $\sum_{n=0}^{T-1} s_{3}^{2}=0$ is auto-correlation of $s_{3}$. Having the thresholds, the receiver detects the received symbols.

$$
r_{i}= \begin{cases}1 & \text { if } \sum_{n=0}^{T-1} r(t) \times \sum_{n=0}^{T-1} s_{1} \geq T h_{1} \\ 0 & \text { else if } \sum_{n=0}^{T-1} r(t) \times \sum_{n=0}^{T-1} s_{2} \geq T h_{2} \\ \text { No-data } & \text { otherwise }\end{cases}
$$

Where, $r_{i}$ is the $i$ th detected symbol at the receiver.

\section{Simulation Results for 3ASK Modulation}

We do the simulation for $0 \%, 10 \%, 40 \%$, and $80 \%$ RH. For each case we consider transmitter-receiver distances of $11.5 \mathrm{~cm}$ and $66.5 \mathrm{~cm}$. Figures 4.18 and 4.19 show BER vs. $\mathrm{E}_{\mathrm{b}} / \mathrm{N}_{0}$ for transmitter-receiver distance of 11.5 and $66.5 \mathrm{~cm}$, respectively.

When the $\mathrm{RH}$ is $0 \%, \mathrm{BER}$ is almost the same as transmitter-receiver distance in increased. However, as the RH is increased, transmitter-receiver distance plays a significant role in BER. For example, when $\mathrm{RH}$ is $40 \%$ we get BER of $10^{-5}$ when $\mathrm{E}_{\mathrm{b}} / \mathrm{N}_{0}$ is $\sim-90 \mathrm{~dB}$ for transmitter-receiver distance is $11.5 \mathrm{~cm}$, whereas for transmitter-receiver distance of $66.5 \mathrm{~cm}$ we get BER of $10^{-5} \mathrm{~dB}$ when $\mathrm{E}_{\mathrm{b}} / \mathrm{N}_{0}$ is $\sim+60 \mathrm{~dB}$. It should be noted that we do not show cases where BER $<10^{-6}$. Also, when transmitter-receiver distance is $66.5 \mathrm{~cm}$ with $80 \% \mathrm{RH}$, the signal is attenuated a great deal more and as a result it is not detectable at the receiver. In this case, since the BER is larger than $10^{-1}$ for all $\mathrm{E}_{\mathrm{b}} / \mathrm{N}_{0}$ we have ignored it. 


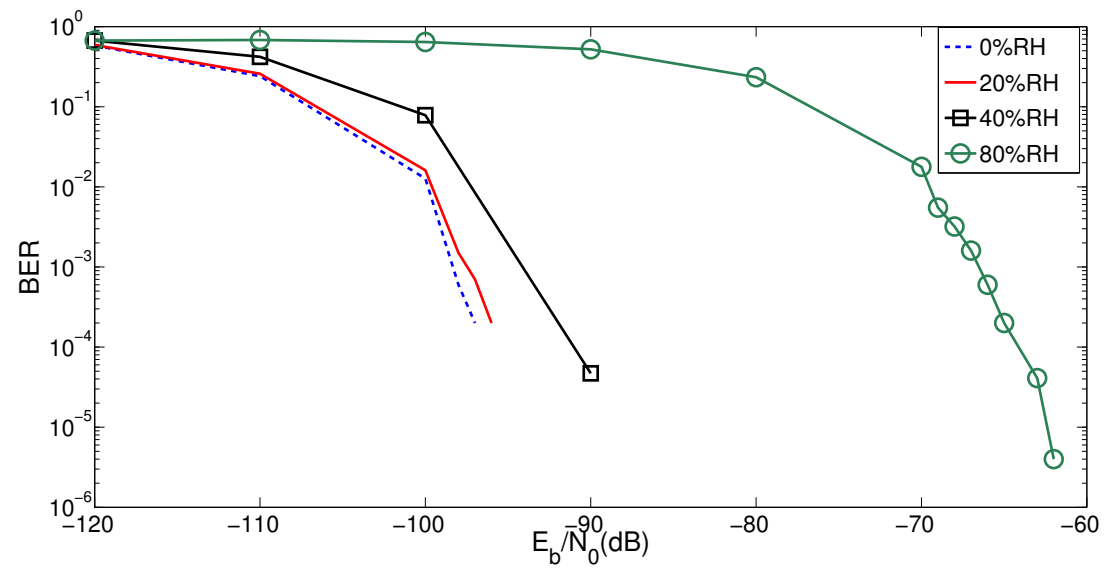

Figure 4.18: BER vs $\mathrm{E}_{\mathrm{b}} / \mathrm{N}_{0}$ corresponding to $3 \mathrm{ASK}$ modulation for different RH levels and transmitter-receiver distance of $11.5 \mathrm{~cm}$.

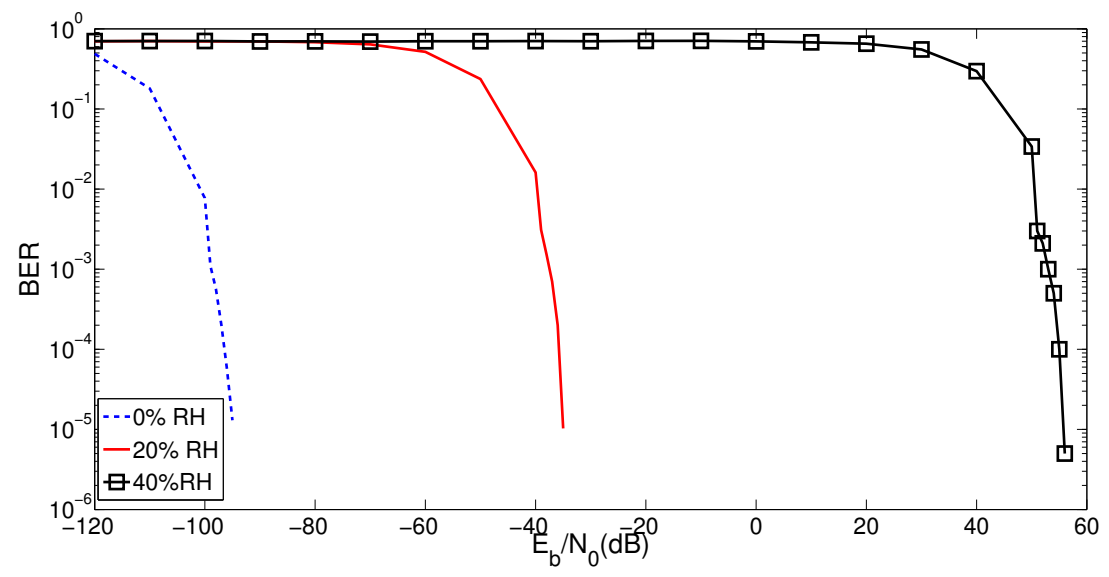

Figure 4.19: BER vs $\mathrm{E}_{\mathrm{b}} / \mathrm{N}_{0}$ corresponding to $3 \mathrm{ASK}$ modulation for different $\mathrm{RH}$ levels and transmitter-receiver distance of $66.5 \mathrm{~cm}$.

\subsubsection{ASK Modulation}

In 5 ASK modulation we encode 2 bits per symbol. Therefore, we need to have 4 different amplitudes and since the modulation is non-coherent we need an extra amplitude that correspond to the case where "No-data" is sent. As a result, we call this modulation 5ASK. 
Lets assume that symbols $s_{1}, s_{2}, \cdots, s_{5}$ correspond to symbols 1 through 5 where the corresponding bit sequences are 10, 00, 01, 11, and "No-data", respectively. Lets assume that $a$ is the amplitude for sending symbol $s_{1}, b$ is the amplitude for sending symbol $s_{2}, c$ is the amplitude for sending symbol $s_{3}, d$ is the amplitude for sending symbol $s_{4}$, and 0 is the amplitude for sending symbol $s_{5}$. Since all of these symbols have the same probability of occurrence, we calculate the amplitude of each symbol from the following equation:

$$
\begin{aligned}
\mathrm{E}[\text { power }] & =\frac{1}{5} \times a^{2}+\frac{1}{5} \times b^{2}+\frac{1}{5} \times c^{2}+\frac{1}{5} \times d^{2}+\frac{1}{5} \times 0=P, \\
d & =\frac{1}{4} a, c=\frac{1}{2} a, b=\frac{3}{4} a
\end{aligned}
$$

Note that, we set amplitudes such that their difference become maximum to get a better detection at receiver. Therefore, we can find the amplitudes corresponding to each symbol as below.

$$
\begin{aligned}
a & =2 \sqrt{\frac{2}{3} P} \\
b & =\frac{3}{2} \sqrt{\frac{2}{3} P} \\
c & =\sqrt{\frac{2}{3} P} \\
d & =\frac{1}{2} \sqrt{\frac{2}{3} P}
\end{aligned}
$$

Where, $P$ is the signal's power at the transmitter.

\section{Non-Coherent Detection of 5ASK Modulation}

As in the case of $3 \mathrm{ASK}$, first we calculate the Fourier transform of the received signal $r(t)$ to get $R(f)$. Then we divide it by the transfer function of the channel $H(f)$ to minimize the effect of channel impairments. We call the new signal in time domain $\dot{r}(t)$. 
If the prototype signal corresponding to symbol 1 is $s_{1}$, the prototype signal corresponding to symbol 2 is $s_{2}$, the prototype signal corresponding to symbol 3 is $s_{3}$, corresponding prototype signal to symbol 4 is $s_{4}$, and finally the corresponding prototype signal to the case where "No-data" is sent is $s_{5}$, we find thresholds in the receiver from $(4.21)$ to $(4.24)$ :

$$
\begin{gathered}
T h_{1}=\left(\sum_{n=0}^{T-1} s_{1}^{2}+\sum_{n=0}^{T-1} s_{2}^{2}\right) / 2 \\
T h_{2}=\left(\sum_{n=0}^{T-1} s_{2}^{2}+\sum_{n=0}^{T-1} s_{3}^{2}\right) / 2 \\
T h_{3}=\left(\sum_{n=0}^{T-1} s_{3}^{2}+\sum_{n=0}^{T-1} s_{4}^{2}\right) / 2 \\
T h_{4}=\left(\sum_{n=0}^{T-1} s_{4}^{2}+\sum_{n=0}^{T-1} s_{5}^{2}\right) / 2=\left(\sum_{n=0}^{T-1} s_{4}^{2}\right) / 2
\end{gathered}
$$

Having the thresholds, the receiver detect the received symbol $r_{i}$ as follows.

$$
r_{i}= \begin{cases}10 & \text { if } \sum_{n=0}^{T-1} \dot{r}(t) \times \sum_{n=0}^{T-1} s_{1} \geq T h_{1}, \\ 00 & \text { else if } \sum_{n=0}^{T-1} r(t) \times \sum_{n=0}^{T-1} s_{2} \geq T h_{2} \\ 01 & \text { else if } \sum_{n=0}^{T-1} r(t) \times \sum_{n=0}^{T-1} s_{3} \geq T h_{3}, \\ 11 & \text { else if } \sum_{n=0}^{T-1} r(t) \times \sum_{n=0}^{T-1} s_{4} \geq T h_{4} \\ \text { No-data } & \text { otherwise }\end{cases}
$$

\section{Simulation Results for 5ASK Modulation}

As in the case of 3ASK modulation, we do the simulation for RH levels of $0 \%, 10 \%$, $40 \%$, and $80 \%$. For each case we consider transmitter-receiver distance of $11.5 \mathrm{~cm}$ and $66.5 \mathrm{~cm}$. Figures 4.20 and 4.21 show BER vs. $\mathrm{E}_{\mathrm{b}} / \mathrm{N}_{0}$ for transmitter-receiver 


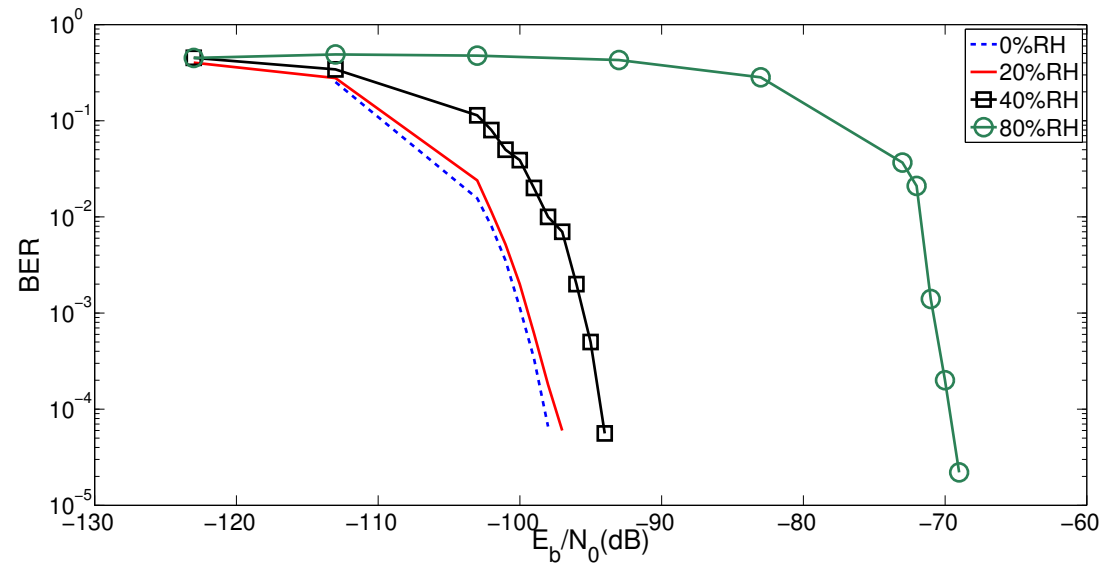

Figure 4.20: BER vs $\mathrm{E}_{\mathrm{b}} / \mathrm{N}_{0}$ corresponding to $5 \mathrm{ASK}$ modulation for different $\mathrm{RH}$ levels and transmitter-receiver distance of $11.5 \mathrm{~cm}$.

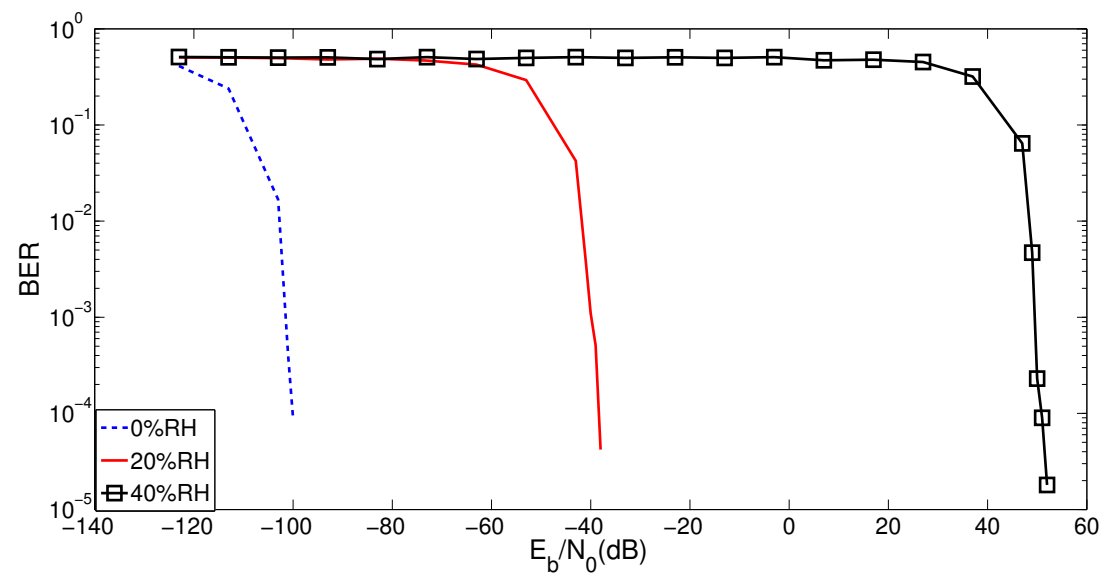

Figure 4.21: BER vs $\mathrm{E}_{\mathrm{b}} / \mathrm{N}_{0}$ corresponding to 5ASK modulation for different RH levels and transmitter-receiver distance of $66.5 \mathrm{~cm}$.

distances of 11.5 and $66.5 \mathrm{~cm}$, respectively. We get the same conclusion as in the case of 3ASK modulation. That is, for higher RH levels increasing transmitterreceiver distance has a significant effect on the BER at the receiver. Note that, in 5ASK modulation we have twice as many bits per symbol as in the case of 3ASK. Therefore, we get slightly lower $\mathrm{E}_{\mathrm{b}} / \mathrm{N}_{0}$ for $5 \mathrm{ASK}$ modulation comparing to $3 \mathrm{ASK}$ 
modulation.

\subsubsection{ASK Modulation Enhancement}

As we discussed earlier, water absorption plays an important role in atmospheric attenuation in the $\mathrm{THz}$ domain. To get better results, we decided to exclude the frequency bands that are attenuated drastically as the humidity increases. Here the number of bands that we exclude is constant and does not change corresponding to humidity level or transmitter-receiver distance. Deleting these bands from the detection process can enhance the result in some cases.

\section{ASK Modulation Enhancement}

For enhancing 3ASK modulation, transmitter transmits data as before. However, the detection procedure is changed to exclude the erroneous bands from our modulation.

In enhanced 3ASK modulation, we calculate the thresholds in frequency domain instead of time domain as in regular 3ASK modulation. Lets assume that the prototype pulse for sending 1 is $S_{1}$ in frequency domain, the prototype pulse for sending 0 is $S_{2}$ in frequency domain, and the prototype pulse for the case where "No-data" is sent is $S_{3}$ in frequency domain.

Using a filter, the receiver slices each prototype pulse into $n$ frequency bands. Then each of these frequency bands, except for the erroneous bands, is multiplied by its conjugate value and then we add up the result for all bands. The following equations show this procedure.

$$
\begin{aligned}
I_{1}= & \sum_{i=0}^{n-1} S_{1}\left(f-f_{i}, \delta f\right) \times S_{1}^{*}\left(f-f_{i}, \delta f\right) \\
& i \notin\{\text { Erroneous Bands }\}
\end{aligned}
$$




$$
\begin{aligned}
I_{0}= & \sum_{i=0}^{n-1} S_{0}\left(f-f_{i}, \delta f\right) \times S_{0}^{*}\left(f-f_{i}, \delta f\right) \\
& i \notin\{\text { Erroneous Bands }\}
\end{aligned}
$$

In (4.26), $n$ is the total number of bands, $S_{1}\left(f-f_{i}, \delta f\right)$ is the complex field amplitude of the $i$ th slice of $S_{1}$ as function of frequency $f$ and $S_{1}^{*}\left(f-f_{i}, \delta f\right)$ is the conjugate value of $S_{1}\left(f-f_{i}, \delta f\right)$. Having these values, the thresholds are defined as follows:

$$
\begin{gathered}
T h_{1}=\frac{I_{1}+I_{0}}{2} \\
T h_{0}=\frac{I_{0}+0}{2}=\frac{I_{0}}{2}
\end{gathered}
$$

Upon arrival of the signal $R(f)$, first $R(f)$ is divided by the transfer function of the channel $H(f)$ to get $R^{\prime}(f)$. Then the receiver slices $R^{\prime}(f)$ into $n$ bands and each band, except for erroneous bands, is multiplied with the corresponding frequency band of $S_{1}$. The result for all bands are added up together at the end. The same procedure is done for $S_{0}$ as is shown in the following equations.

$$
\begin{aligned}
E_{1}= & \sum_{i=0}^{n-1} R^{\prime}\left(f-f_{i}, \delta f\right) \times S_{1}^{*}\left(f-f_{i}, \delta f\right) \\
& i \notin\{\text { Erroneous Bands }\} \\
E_{0}= & \sum_{i=0}^{n-1} R^{\prime}\left(f-f_{i}, \delta f\right) \times S_{0}^{*}\left(f-f_{i}, \delta f\right) \\
& i \notin\{\text { Erroneous Bands }\}
\end{aligned}
$$

Then the receiver compares these values with the thresholds to detect the received symbol $r_{i}$.

$$
r_{i}= \begin{cases}1 & \text { if } E_{1} \geq T h_{1} \\ 0 & \text { else if } E_{0} \geq T h_{0} \\ \text { Nothing has been sent } & \text { otherwise }\end{cases}
$$




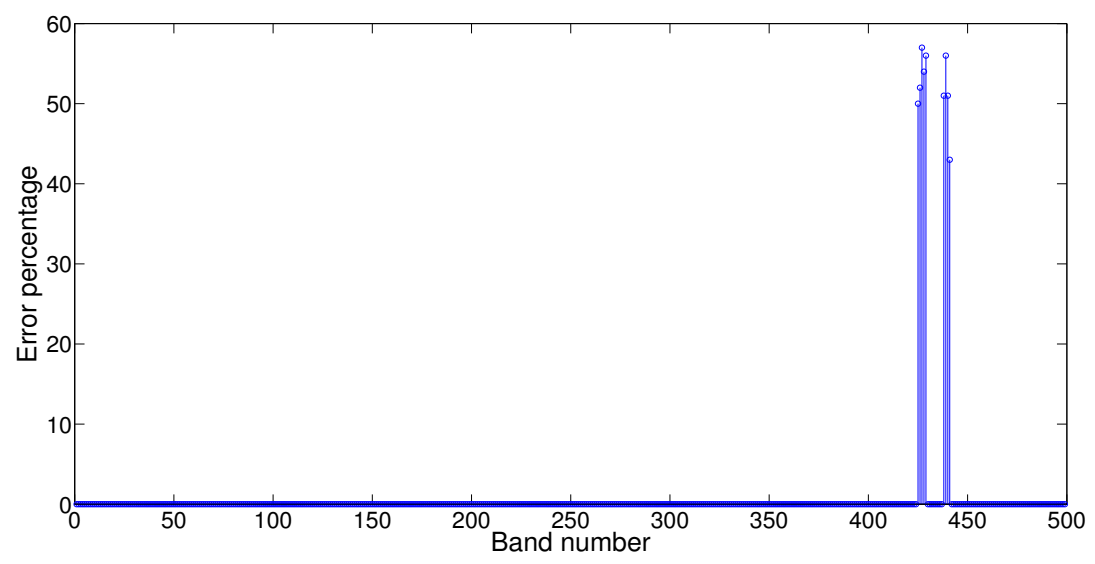

Figure 4.22: Error percentage of all bands when $\mathrm{RH}=40 \%$, transmitter-receiver distance is $66.5 \mathrm{~cm}$, and $\mathrm{E}_{\mathrm{b}} / \mathrm{N}_{0}=0$.

\section{Enhanced 3ASK Simulation Results}

In enhanced 3ASK modulation, we find the bands that are causing most of the error at the receiver. For that we divide the frequency domain to 500 bands and we use FD-DBSPK modulation as we explained earlier. We set $\mathrm{RH}$ to $40 \%$ and transmitter-receiver distance to $66.5 \mathrm{~cm}$. Figure 4.22 shows the error percentage of all bands in FD-DBPSK modulation when $\mathrm{E}_{\mathrm{b}} / \mathrm{N}_{0}=0$. It should be noted that having more frequency slices results in a better precision in excluding the erroneous bands. However, the execution time will be increased.

The result show that symbols corresponding to 9 bands are detected inaccurately $50 \%$ of the time while the other 491 bands do not cause any detection error. Figure 4.23 shows these 9 erroneous bands with the error percentage that they cause.

In our simulation, we divide the frequency domain into 500 bands using the raised-cosine filter with roll-off factor of 0.5 . Therefore, $n$ is equal to 500 in the corresponding equations. 


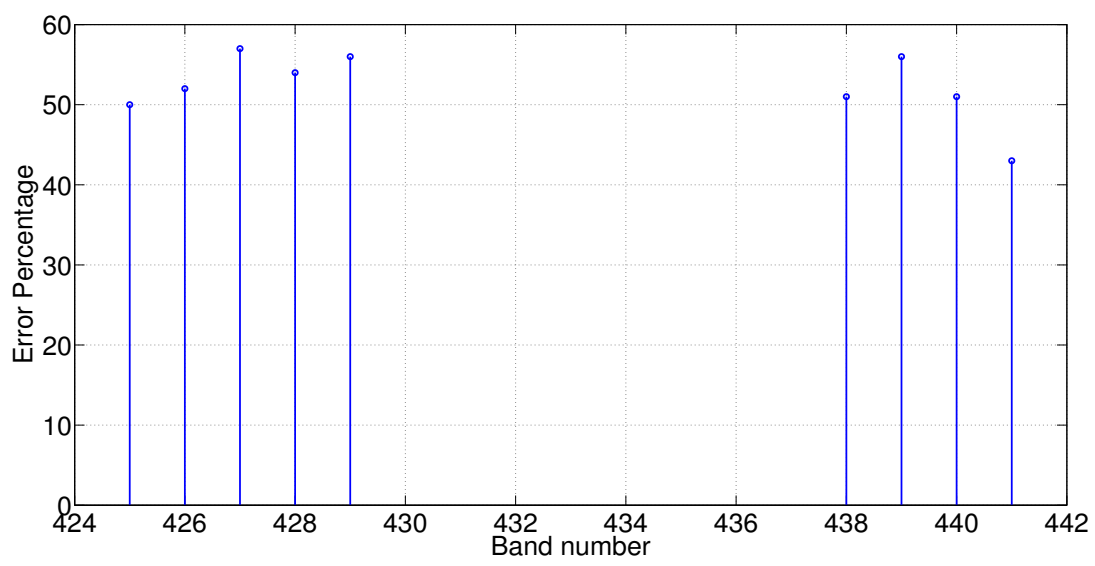

Figure 4.23: Error percentage of erroneous bands when $\mathrm{RH}=40 \%$, transmitter-receiver distance is $66.5 \mathrm{~cm}$, and $\mathrm{E}_{\mathrm{b}} / \mathrm{N}_{0}=0$.

Figures 4.24 and 4.25 show the output of the enhanced 3ASK for different humidity levels for transmitter-receiver distances of 11.5 and $66.5 \mathrm{~cm}$, respectively where the 9 bands from Figure 4.23 have been excluded.

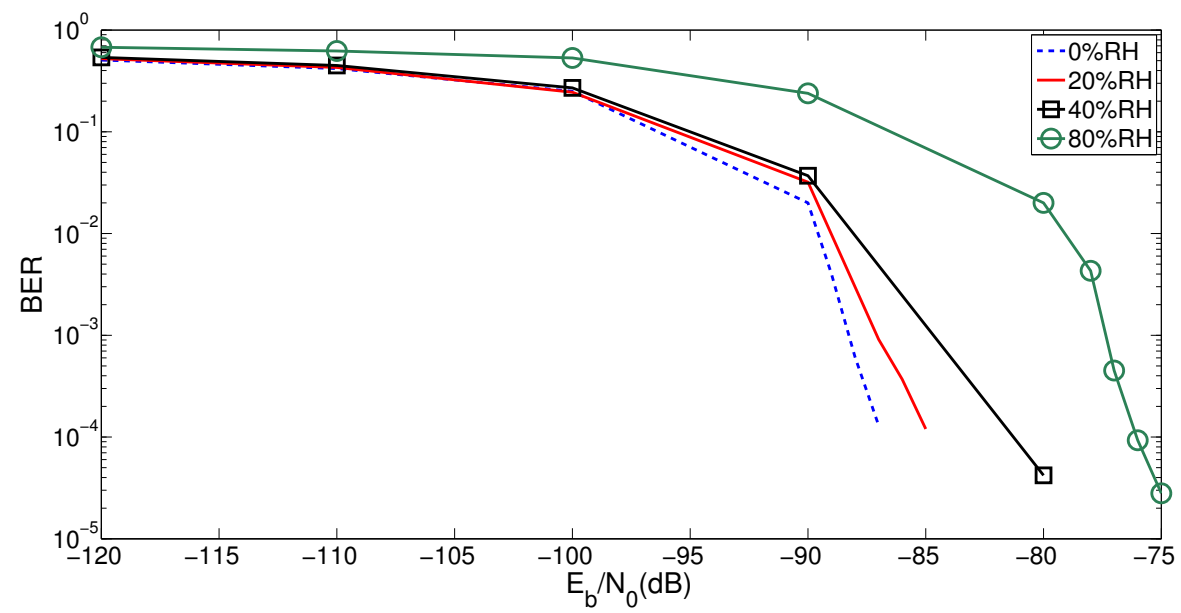

Figure 4.24: Enhanced BER based on 3ASK modulation for transmitter-receiver distance of $11.5 \mathrm{~cm}$ 


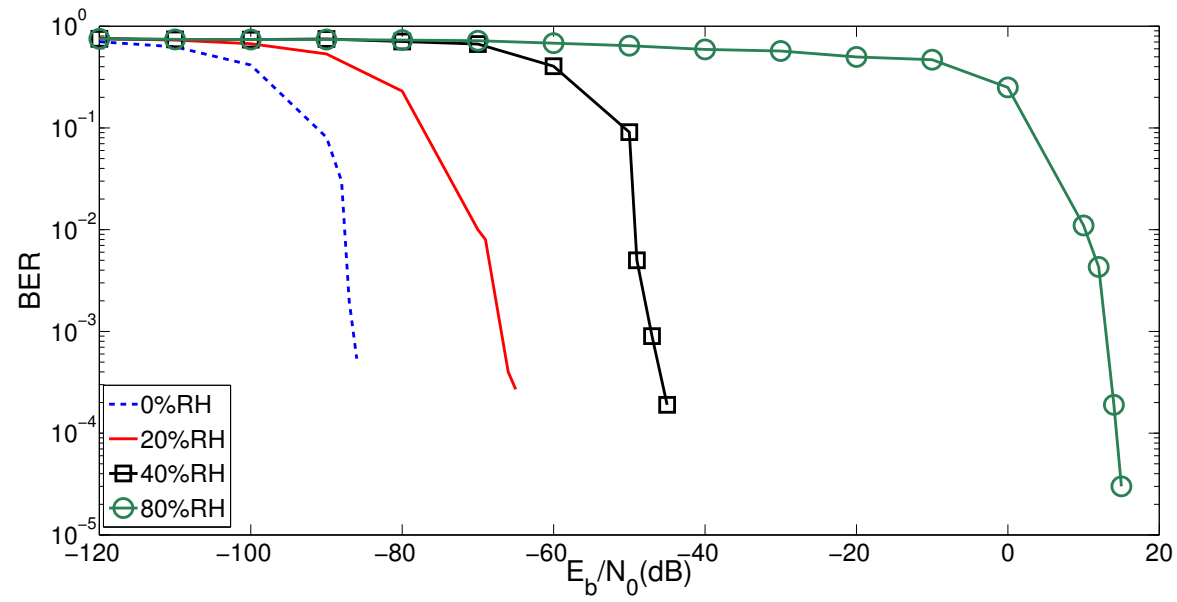

Figure 4.25: Enhanced BER based on 3ASK modulation for transmitter-receiver distance of $66.5 \mathrm{~cm}$

Comparing Figures 4.24 and 4.18, we find that in enhanced 3ASK modulation, BER corresponding to $80 \% \mathrm{RH}$ is decreased. That is in regular 3ASK modulation, we get BER of $\sim 10^{-6}$ for $\mathrm{E}_{\mathrm{b}} / \mathrm{N}_{0}$ of $-61 \mathrm{~dB}$. Whereas, in enhanced 3ASK modulation, we get the same BER for $\mathrm{E}_{\mathrm{b}} / \mathrm{N}_{0}$ of $-74 \mathrm{~dB}$. However, the BER corresponding to $0 \%, 20 \%$, and $40 \% \mathrm{RH}$ has been increased in enhanced modulation. The reason for this degradation is that when $\mathrm{RH}$ is not very high and transmitter-receiver distance is short, erroneous bands are not attenuated that much and as a result they do not cause detection errors. Therefore, deleting them decreases the received signal power and lowers the $\mathrm{E}_{\mathrm{b}} / \mathrm{N}_{0}$ resulting in a higher BER.

Comparing Figures 4.25 and 4.19, we find that for longer transmitter-receiver distance of $66.5 \mathrm{~cm}$, the result is improved a lot in enhanced modulation. Even for the case of $80 \% \mathrm{RH}$ the signal is detectable at the receiver with $\mathrm{BER}<10^{-6}$ when $\mathrm{E}_{\mathrm{b}} / \mathrm{N}_{0}>19 \mathrm{~dB}$. The reason is that erroneous bands are attenuated a lot and they cause error in detection, excluding them from the detection process will result in a better $\mathrm{BER}$ at the receiver. However, for $\mathrm{RH}$ of $0 \%$ the result in enhanced 
modulation is slightly degraded.

\section{ASK Modulation Enhancement}

In enhanced 5ASK modulation, the transmitter uses the same equations as in regular 5ASK. However, the receiver's equations are modified to eliminate the erroneous bands from calculation.

As in the case of adaptive 3ASK modulation, each spectral prototype signal is divided into $n$ bands. Then for each frequency band, we multiply the prototype signal with its conjugate, and the result is added up.

$$
\begin{aligned}
I_{1}= & \sum_{i=0}^{n-1} S_{1}\left(f-f_{i}, \delta f\right) \times S_{1}^{*}\left(f-f_{i}, \delta f\right) \\
& i \notin\{\text { Erroneous Bands }\} \\
I_{2}= & \sum_{i=0}^{n-1} S_{2}\left(f-f_{i}, \delta f\right) \times S_{2}^{*}\left(f-f_{i}, \delta f\right) \\
& i \notin\{\text { Erroneous Bands }\} \\
I_{3}= & \sum_{i=0}^{n-1} S_{3}\left(f-f_{i}, \delta f\right) \times S_{3}^{*}\left(f-f_{i}, \delta f\right) \\
& i \notin\{\text { Erroneous Bands }\} \\
I_{4}= & \sum_{i=0}^{n-1} S_{4}\left(f-f_{i}, \delta f\right) \times S_{4}^{*}\left(f-f_{i}, \delta f\right) \\
& i \notin\{\text { Erroneous Bands }\}
\end{aligned}
$$

Here, $S_{1}-S_{5}$ are prototype signals corresponding to symbols $1-5$. Having these values, we define thresholds from the below equations.

$$
T h_{1}=\frac{I_{1}+I_{2}}{2}
$$




$$
\begin{aligned}
& T h_{2}=\frac{I_{2}+I_{3}}{2} \\
& T h_{3}=\frac{I_{3}+I_{4}}{2} \\
& T h_{4}=\frac{I_{4}+0}{2}
\end{aligned}
$$

To detect the received signal $R(f)$, it is first divided by the transfer function of the channel $H(f)$. Then the result, $R^{\prime}(f)$, is sliced into $n$ bands and the complex amplitude value of each band, except for the erroneous ones, is multiplied by the corresponding complex amplitude value of each prototype signal available at the receiver.

$$
\begin{aligned}
E_{1}= & \sum_{i=0}^{n-1} R^{\prime}\left(f-f_{i}, \delta f\right) \times S_{1}^{*}\left(f-f_{i}, \delta f\right) \\
& i \notin\{\text { Erroneous Bands }\} \\
E_{2}= & \sum_{i=0}^{n-1} R^{\prime}\left(f-f_{i}, \delta f\right) \times S_{2}^{*}\left(f-f_{i}, \delta f\right) \\
& i \notin\{\text { Erroneous Bands }\} \\
E_{3}= & \sum_{i=0}^{n-1} R^{\prime}\left(f-f_{i}, \delta f\right) \times S_{3}^{*}\left(f-f_{i}, \delta f\right) \\
& i \notin\{\text { Erroneous Bands }\} \\
& E_{4}=\sum_{i=0} R^{\prime}\left(f-f_{i}, \delta f\right) \times S_{4}^{*}\left(f-f_{i}, \delta f\right) \\
& i \notin\{\text { Erroneous Bands }\}
\end{aligned}
$$


receiver detects the received symbol $r_{i}$ by comparing the results with thresholds.

$$
r_{i}= \begin{cases}10 & \text { if } E_{1} \geq T h_{1} \\ 00 & \text { else if } E_{2} \geq T h_{2} \\ 01 & \text { else if } E_{3} \geq T h_{3} \\ 11 & \text { else if } E_{4} \geq T h_{4} \\ \text { No data has been sent } & \text { otherwise }\end{cases}
$$

\section{Enhanced 5ASK Simulation Results}

As in the case of adaptive 3ASK, we set the number of frequency slices, $n$, to 500. That is the frequency domain of the prototype signals and the received signal are divided into 500 frequency bands. Since the bandwidth is $2 \mathrm{THz}$, each sliced frequency band has bandwidth of $4 \mathrm{GHz}$. We used the raised cosine filter with rolloff factor of 0.5 to exclude erroneous bands from the detection process. Figures 4.26 and 4.27 show the output of the enhanced 5ASK modulation scheme for different humidity levels for transmitter-receiver distances of 11.5 and $66.5 \mathrm{~cm}$, respectively. 


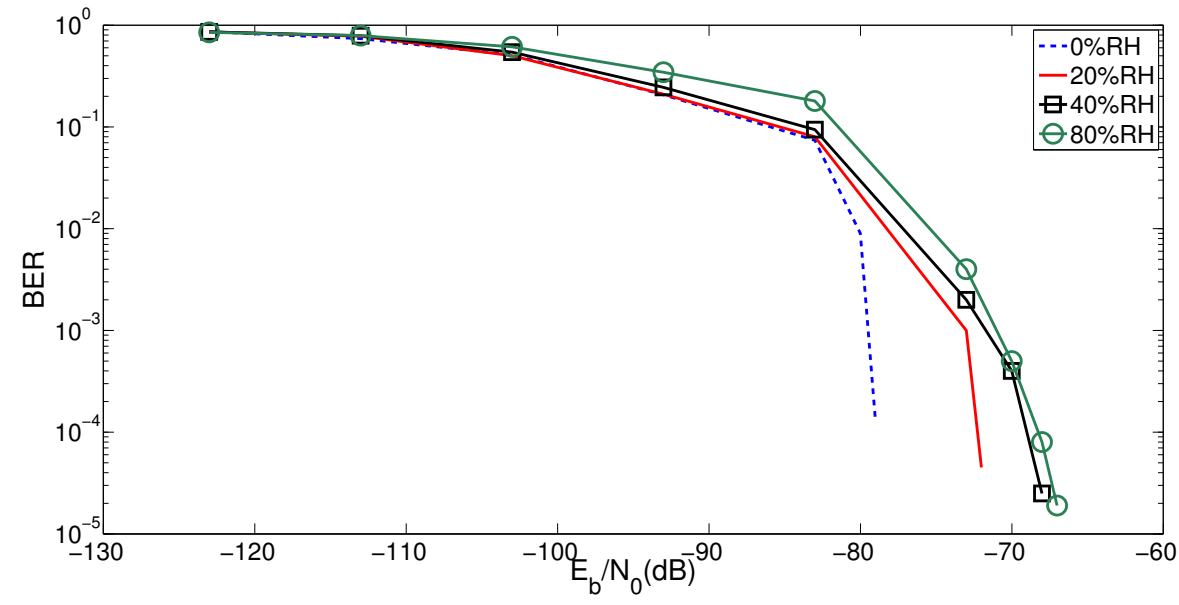

Figure 4.26: Enhanced BER based on 5ASK modulation for transmitter-receiver distance of $11.5 \mathrm{~cm}$

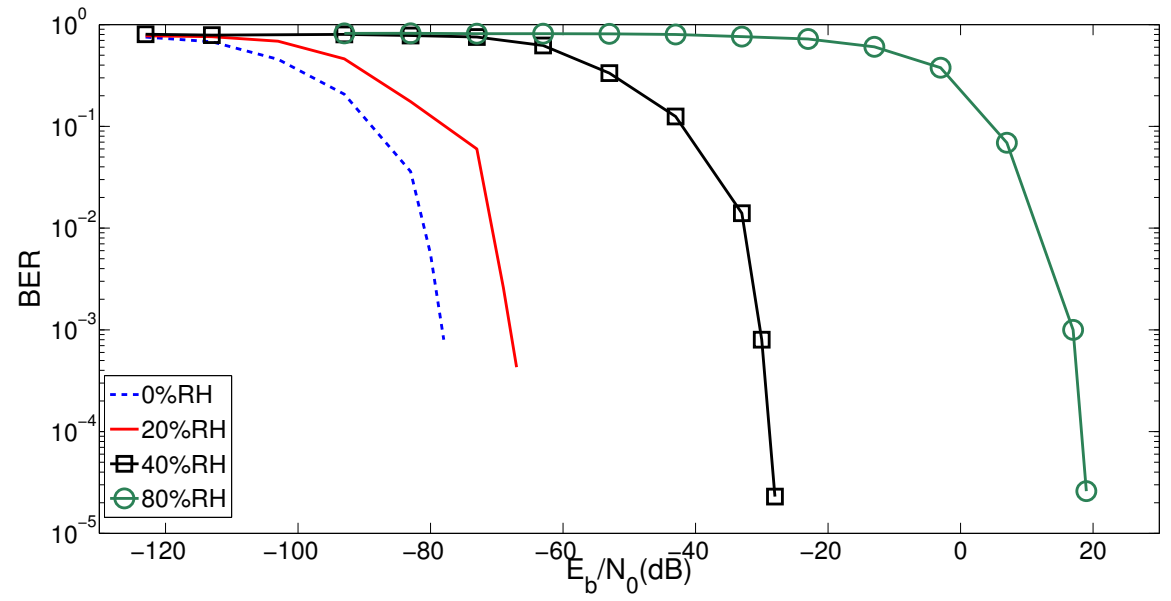

Figure 4.27: Enhanced BER based on 5ASK modulation for transmitter-receiver distance of $66.5 \mathrm{~cm}$

Comparing these figures with Figures 4.20 and 4.21 shows that for shorter transmitter-receiver distance of $11.5 \mathrm{~cm}$ the $\mathrm{BER}$ result of $80 \% \mathrm{RH}$ is not changed while the result for lower humidity levels of $0 \%, 20 \%$, and $40 \%$ are degraded as by 
excluding erroneous bands the received $\mathrm{E}_{\mathrm{b}} / \mathrm{N}_{0}$ is decreased at the receiver.

For longer transmitter-receiver distance of $66.5 \mathrm{~cm}$ we see a great enhancement for $20 \%, 40 \%$, and $80 \% \mathrm{RH}$ while the BER result for $0 \%$ is slightly degraded.

\subsubsection{Data Rate for Cities Based on Enhanced ASK Modulation}

For cities in Table 4.4, we simulate the ASK modulation methods to find an average bit rate vs distance based on (4.11). Figures 4.28 and 4.29 show the result of simulating enhanced 3ASK and 5ASK modulations, respectively.

As the figures show, we get better result when $\mathrm{RH}$ and/or transmitter-receiver distance is smaller. Also, for each RH level the receiver can detect the symbol to some specific transmitter-receiver distance and after that point the signal is attenuated such that it is not detectable.

\section{SUMMARY}

In this section we studied communication using a pulsed $\mathrm{THz}$ system and directional channels. We simulated data transmission corresponding to different humidity levels for ASK modulation as well as FD-DPSK modulation methods. We also calculated the average achievable data rate for different cities around US with different relative humidities. Moreover, we described how multiple transmitters can be used to achieve terabit data rates as well as support multiple users simultaneously in FD-DPSK modulation. In the next chapter we will see how we can support multiple users at the same time using omni-directional THz channels. 


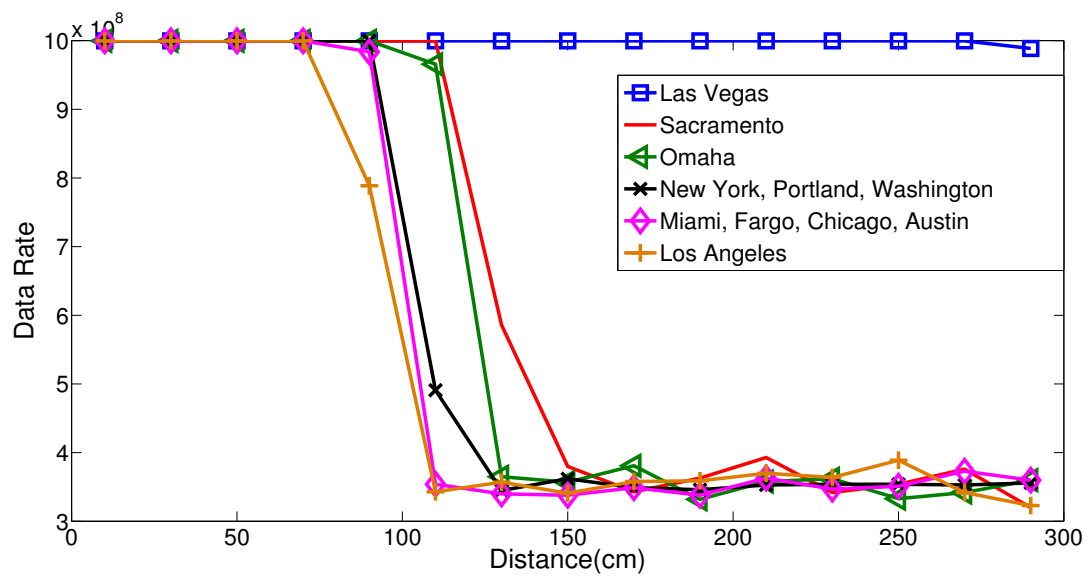

(a)

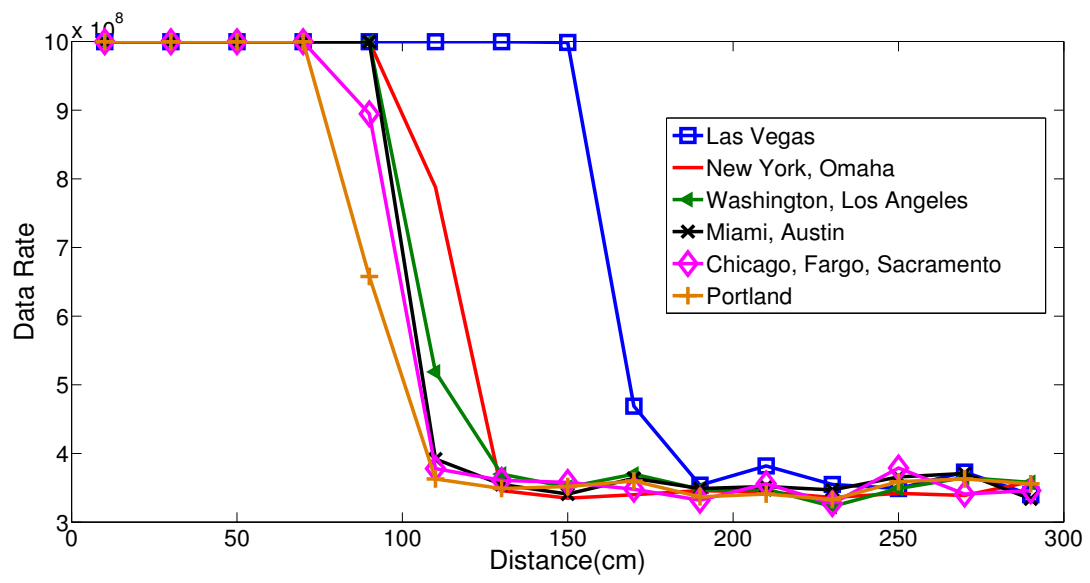

(b)

Figure 4.28: Enhanced 3ASK modulation data rate vs distance (cm) for cities in (a) summer and (b) winter. 


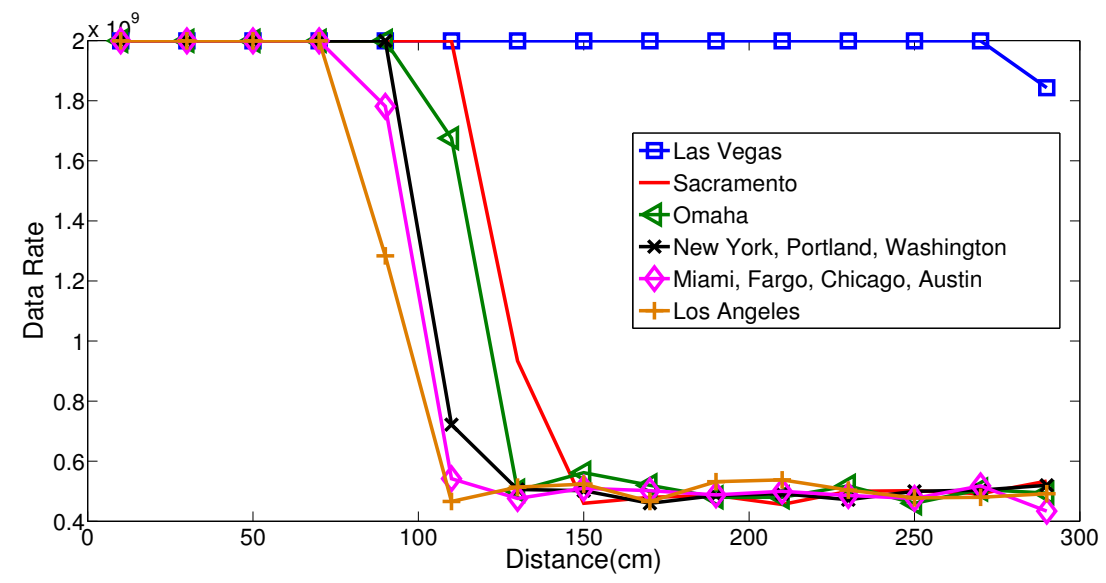

(a)

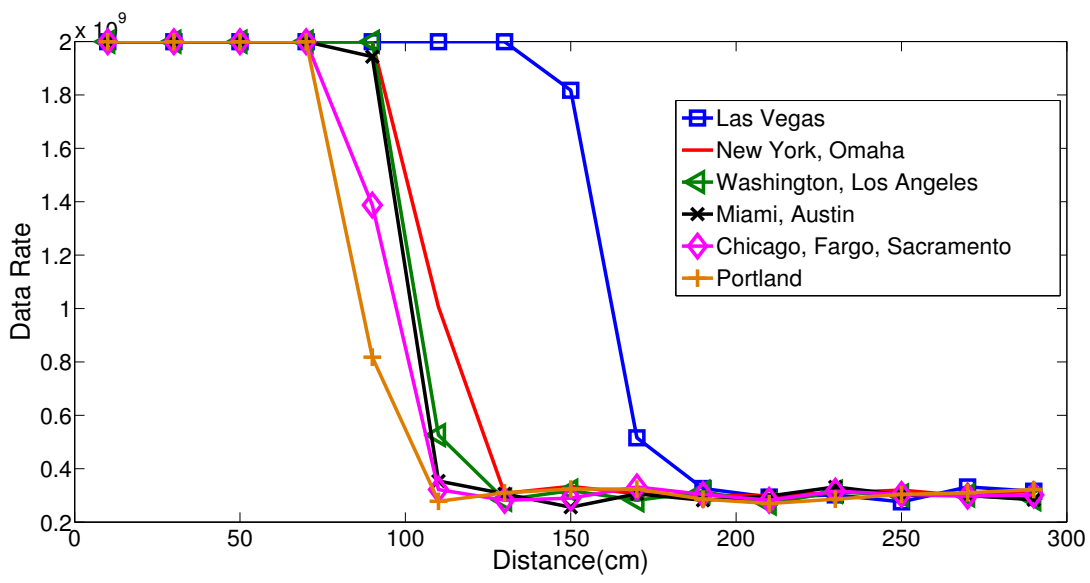

(b)

Figure 4.29: Enhanced 5ASK modulation data rate vs distance (cm) for cities in (a) summer and (b) winter. 
Chapter 5

\section{OMNI-DIRECTIONAL COMMUNICATION MODELS}

In this chapter we study ideal omni-directional terahertz channels. However, unlike

the analysis in Chapter 4, we assume a continuous wave (CW) system rather than a pulsed system. Continuous waves have smaller bandwidth than pulsed signals that we use in directional channels. Therefore, the $\mathrm{THz}$ transmitter and receiver can be tuned to a specific frequency that is not attenuated drastically by water vapor molecules. As a result, the transmitted signal can reach receivers that are placed relatively further away from the transmitter. As a result CW can be used in omni-directional channels to avoid the limitations of a directional channel.

In the following sections we first derive the omni-directional channel model based on our measurements from Chapter 3. Then we analyze the performance of standard methods. Finally, we develop two rate adaptation algorithms based on the result of the simulations.

\section{OMNI-DIRECTIONAL CHANNEL CHARACTERIZATION AND MODELING}

To characterize the $\mathrm{THz}$ omni-directional channel we find the total attenuation coefficient $K(f)$ from (3.11). As we discussed earlier, according to the Beer-Lambart law the power of the signal decreases exponentially with transmitter-receiver distance. Also, based on Friis equation, in the case of omni-directional antennas, the signal also suffers the effect of free space path loss. Therefore, the transfer function 


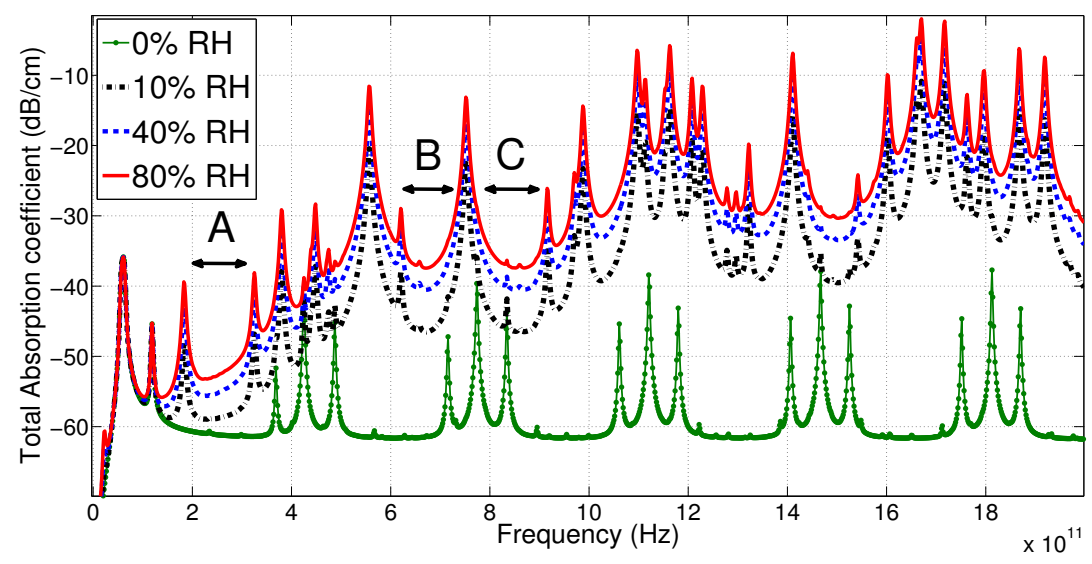

Figure 5.1: Atmospheric attenuation coefficient for multiple relative humidity levels and the three channels with relatively less attenuations.

for our channel is as follows.

$$
H(f)=\frac{\lambda}{4 \pi d} \times e^{-(i 2 \pi f \tau+d K(f))}
$$

In this equation, $\lambda$ is the wavelength in $\mathrm{cm}$ for which the transfer function is calculated, $d$ is the transmitter-receiver distance in $\mathrm{cm}, \tau$ is the time in seconds required for the signal to travel through distance $d$.

Figure 5.1 shows atmospheric attenuation coefficient in $\mathrm{dB} / \mathrm{cm}$ as a function of frequency for the range of $0-2 \mathrm{THz}$ for $0 \%, 10 \%, 40 \%$, and $80 \% \mathrm{RH}$ levels. The result shows that there are three windows $\mathrm{A}, \mathrm{B}$, and $\mathrm{C}$ in which the absorption coefficient is smaller compared to the other frequency bands. Hence, we use the center carrier frequencies of $237.5 \mathrm{GHz}$ (channel A), $677.5 \mathrm{GHz}$ (channel B) and 872.5 GHz (channel C) with the bandwidth of $35 \mathrm{GHz}$ in this chapter. Figure 5.2 plots the calculated received power based on the transfer function from (5.1) for these three channels.

Figure 5.3 shows the received signal's power using channel A. The solid line 


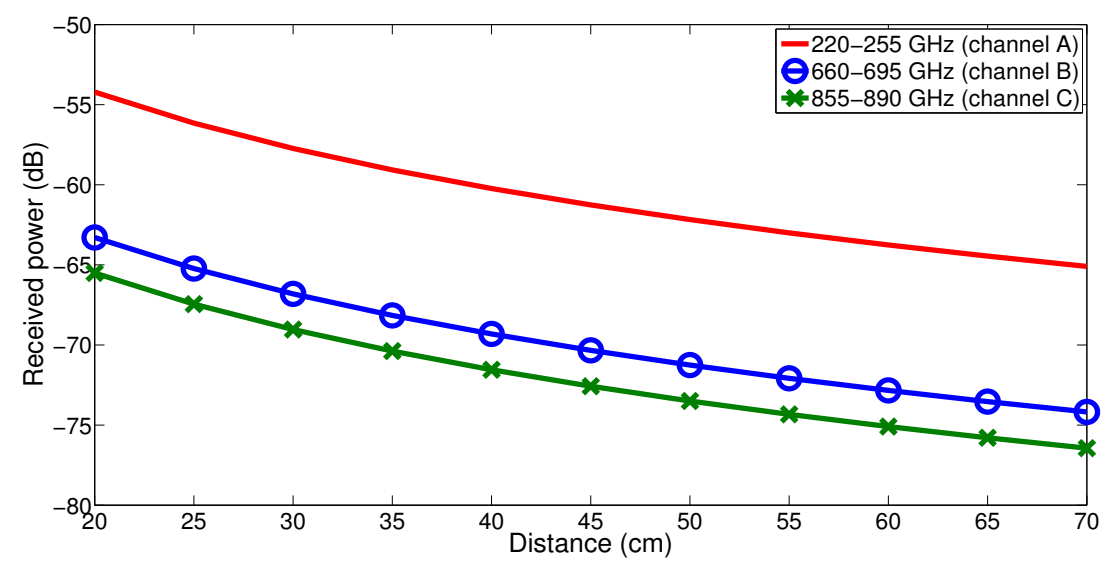

Figure 5.2: Received power of 220-255 GHz (channel A), 660-695 GHz (channel B) and 855-890 GHz (channel C) using omni-directional antennas.

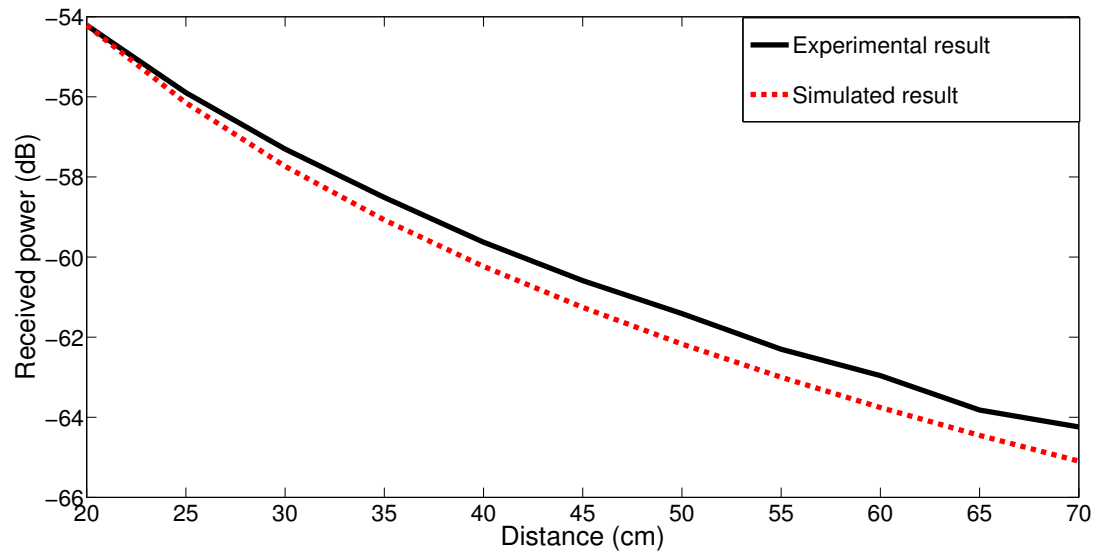

Figure 5.3: Received power of 220-255 GHz (channel A) using omni-directional antennas.

is the result that we get from our lab experiment when we remove the collimating lenses from both the transmitter and the receiver, and the dotted line is the corresponding result that we calculate based on the transfer function from (5.1). 


\section{RATE ADAPTATION ALGORITHMS}

In order to explain the overall problem addressed in this chapter, it is useful to first present the system model. Assume the presence of a terahertz access point (TAP) located in the ceiling so that it has line of sight (LOS) to all the potential users. Let us also assume that there is a wifi link between the users and the TAP. The wifi link is used as the control channel for the terahertz downlink. Thus, the wifi link is used in the normal way to register users into the network and to access the Internet. The only difference is that when sending large amounts of data to the user, the TAP sends the data stream over the terahertz channel rather than the wifi link.

We develop two rate adaptation algorithms in which TAP adapts the modulation order based on the channel parameters. In the first algorithm we use a single channel (A, B, or C) only. Later, in section 5.2.2, we develop a second algorithm that utilizes all three channels (A, B, and C) together. We assume that the wifi channel is used to estimate the distance to each user and the TAP also measures the relative humidity of the air. Using this information, the TAP can compute the expected $\mathrm{E}_{\mathrm{b}} / \mathrm{N}_{0}$ (energy per bit to the spectral noise density) for different users and different modulation schemes. Then it selects the highest order modulation with $\mathrm{BER} \leq \mathrm{BER}_{T h}$, where $\mathrm{BER}_{T h}$ is the maximum BER (bit error rate) that can

be tolerated at each receiver. We use MPSK (phase shift keying) modulation in which $M$ is changed based on the user distance from the TAP.

Our first algorithm is called single channel algorithm (SCA) in which TAP uses a single channel to send data to the users, whereas in the multiple channel algorithm (MCA) TAP uses three channels A, B, and C at the same time to increase the data rate of the system. 


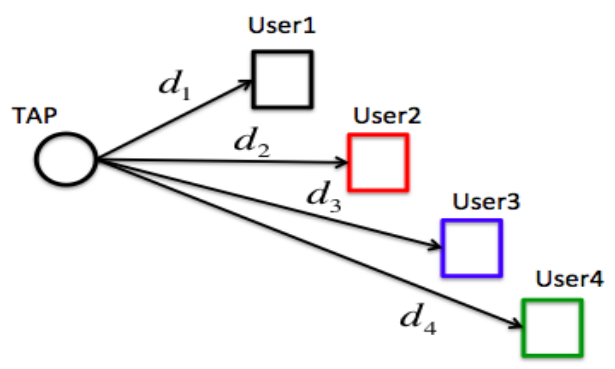

Figure 5.4: System schematic.

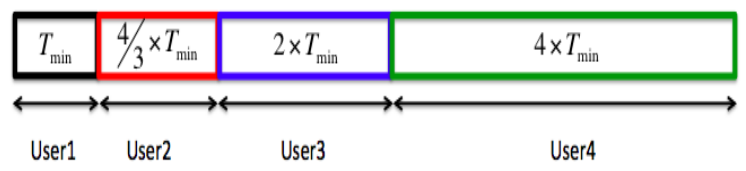

Figure 5.5: Time slots assigned to each user in SCA.

\subsubsection{Single Channel Algorithm (SCA)}

To have a fair connection in which all users can achieve similar data rates, TAP sends data to each user in a time devision multiplexing (TDM) system. For that, TAP assigns the smallest time slot, $T_{m i n}$, to the user that has the highest order

modulation. For users with lower order modulation, TAP selects multiples of $T_{\text {min }}$ such that all users receive almost the same data rate.

Imagine that there are 4 users and based on their distances to TAP, $d_{1}, \cdots, d_{4}$, the received $\mathrm{E}_{\mathrm{b}} / \mathrm{N}_{0}$ for each of them is such that the TAP assigns 16PSK, 8PSK, QPSK, and BPSK modulation to the users, respectively. Figure 5.4 is a schematic of this system and Figure 5.5 shows how TAP assigns time slots to each user in order to maintain fairness in the channel.

In general, the $i$ th user in its time slot receives the upper bound of $b_{i}$ bits of error free information that is equal to the following equation.

$$
b_{i}=\left\lfloor\frac{T_{i}}{T_{s}}\right\rfloor \log _{2} M_{i}-\left[\mathrm{BER} \times\left\lfloor\frac{T_{i}}{T_{s}}\right\rfloor \log _{2} M_{i}\right]
$$


Here, $T_{s}$ is the symbol time, $T_{i}$ is the time slot, and $M_{i}$ is the modulation order that are assigned to the $i$ th user. Therefore, having the total of $I$ users, we can calculate the data rate for each user, $r_{i}$, as follows.

$$
r_{i}=\frac{b_{i}}{\sum_{i=1}^{I} T_{i}}
$$

\subsubsection{Multiple Channel Algorithm (MCA)}

In this algorithm, TAP uses channels A, B, and C simultaneously to send data to users and achieve higher data rate for each user. As we will see later in section 5.3, each channel covers different ranges of TAP-user distances. The reason is that the frequency of channel $\mathrm{A}$ is lower than the other two channels and the lower frequency means longer wavelengths. Also, from (5.1), we saw that signal with

shorter wavelengths attenuated more. Therefore, using channel A, TAP can send data to users with longer distances in comparison with channels B and C. For the same reason channel B covers users with longer distance than channel C.

In MCA, time slots with the same duration $T$ are assigned to all users. In each time slot, TAP sends data to three users using different channels such that fairness is maintained among users and the highest data rate is achieved. The following is the rate adaptation algorithm that is used in MCA.

\section{Multiple Channel Algorithm (MCA)}

1: Assign Counter $=0$ to all users

2: Range $_{A}$ : The longest distance that can be covered using channel A 
3: Range $_{B}$ : The longest distance that can be covered using channel $\mathrm{B}$

4: Range $_{C}$ : The longest distance that can be covered using channel $\mathrm{C}$

5: for each timeSlot do

6: minCounter $_{A}=\operatorname{Min}($ Counter $)$ of users with distances $\leq$ Range $_{A}$

7: Find the user with the longest distance that has Counter $=$ minCounter $_{A}$, assign channel A to the user

8: If a user is found, find the highest order modulation method using channel A that has $\mathrm{BER} \leq \mathrm{BER}_{T h}$ and

9: $\quad$ Add the $\log _{2} M$ corresponding to the selected modulation to the user's Counter

10: minCounter $_{B}=\operatorname{Min}($ Counter $)$ of users with distances $\leq$ Range $_{B}$

11: Find the user with the shortest distance that is in $\left(\right.$ Range $_{C}$, Range $\left._{B}\right]$ and has Counter $=$ minCounter $_{B}$, assign channel B to the user

12: If did not find such user, find the user with the longest distance $\leq$ Range $_{C}$ that has Counter $=$ minCounter $_{B}$, assign channel B to the user

13: If a user is found, find the highest order modulation method using channel $\mathrm{B}$ that has $\mathrm{BER} \leq \mathrm{BER}_{T h}$ and

14: Add the $\log _{2} M$ corresponding to the selected modulation to the user's Counter

15: minCounter $_{C}=\operatorname{Min}($ Counter $)$ of users with distances $\leq$ Range $_{C}$

16: Find the user with the shortest distance that has Counter $=$ minCounter $_{C}$, assign channel $\mathrm{C}$ to the user

17: If a user is found, find the highest order modulation method using channel $\mathrm{C}$ with $\mathrm{BER} \leq \mathrm{BER}_{T h}$ and

18: Add the $\log _{2} M$ corresponding to the selected modulation to the user's Counter 19: end for 
MCA keeps track of the downlink data rate that is assigned to each user with the Counter variable. The upper bound of the received data bits for the $i$ th user is equal to the following equation.

$$
b_{i}=\sum_{t=1}^{K} \sum_{z=1}^{3}\left(\left\lfloor\frac{T}{T_{s}}\right\rfloor \log _{2} M_{i z}-\left[\mathrm{BER}_{i z} \times\left\lfloor\frac{T}{T_{s}}\right\rfloor \log _{2} M_{i z}\right]\right)
$$

Here, $T$ is the duration of each time slot, $K$ is the number of time slots, $M_{i z}$ is the modulation order and $B E R_{i z}$ is the bit error rate for the $i$ th user that is receiving data on channel $z$, where $z=1, z=2$, and $z=3$ correspond to channels $\mathrm{A}, \mathrm{B}$, and $\mathrm{C}$, respectively. Therefore, the data rate for user $i$ is found as follows.

$$
r_{i}=\frac{b_{i}}{\sum_{t=1}^{K} T}
$$

\section{SIMULATION RESULTS}

We choose BPSK, QPSK, 8PSK, and 16PSK modulation in our rate adaptation algorithms and use Matlab for the simulation. The symbol rate of our system is set to $25 \mathrm{GBd} / \mathrm{s}$ and we use the raised cosine filter with rolloff factor 0.4 . Figure 5.6 shows BER corresponding to all modulation methods for received $E_{b} / N_{0}(d B)$ levels. For the sake of comparison, we also show the BER vs $E_{b} / N_{0}$ for $8 Q A M$ and 16QAM methods. As the figure shows, QAM methods have higher BER for the same $\mathrm{E}_{\mathrm{b}} / \mathrm{N}_{0}$ in comparison with PSK modulation methods. Therefore, we only use PSK methods in our rate adaptation algorithms.

We set the $\mathrm{BER}_{T h}$ to $10^{-4}$ and we select the highest order modulation that has $B E R<\mathrm{BER}_{T h}$ for the received $\mathrm{E}_{\mathrm{b}} / \mathrm{N}_{0}$.

Based on the channel model from 5.1 and the transmitter power of $37 \mathrm{~mW}$, we calculate the received $\mathrm{E}_{\mathrm{b}} / \mathrm{N}_{0}$ and the corresponding BER for TAP-user distances 


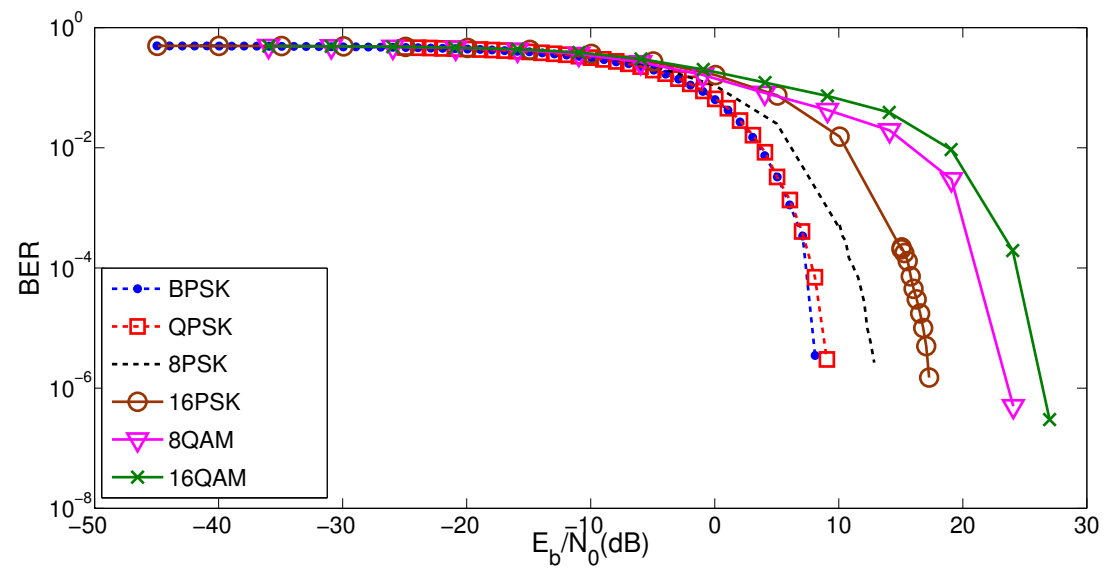

Figure 5.6: Bit error rate $(\mathrm{BER})$ vs received $\mathrm{E}_{\mathrm{b}} / \mathrm{N}_{0}$ for modulation methods.

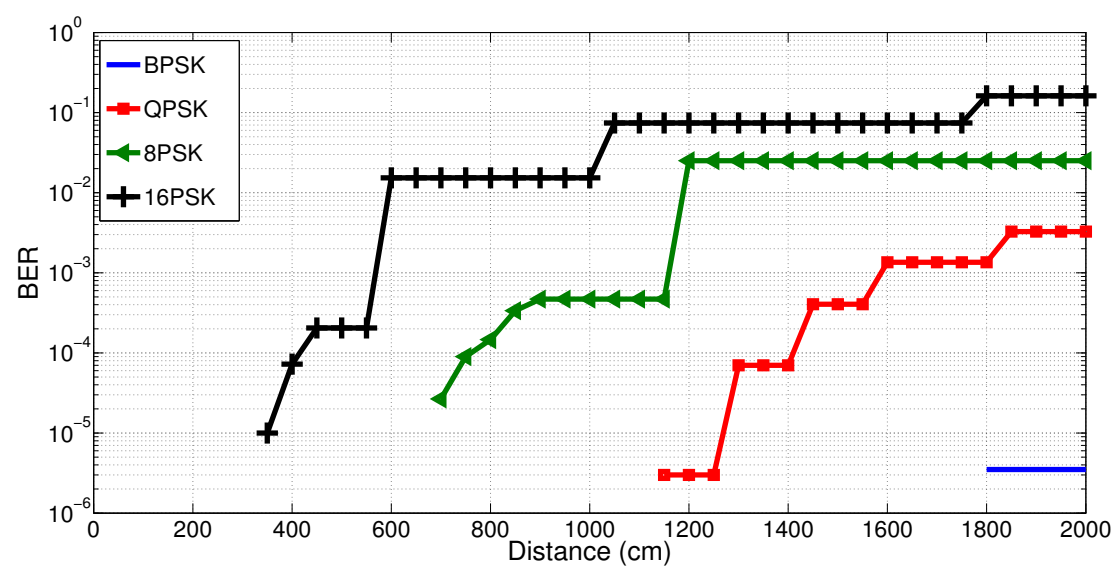

Figure 5.7: Bit error rate vs distance $(\mathrm{cm})$ for modulation methods using channel A for transmission.

of 100-2000 cm for channels A, B, and C. Figures 5.7-5.9 show BER vs distance for the modulation methods when we use channel A, B, or C, respectively. We should note that BER less than $10^{-6}$ is not shown in the figures.

As the figures show, and we mentioned earlier, the received power is attenuated more when we use higher frequencies. Therefore, channel A gives the best result, whereas channels B and C have higher BER for the same TAP-user distance. 


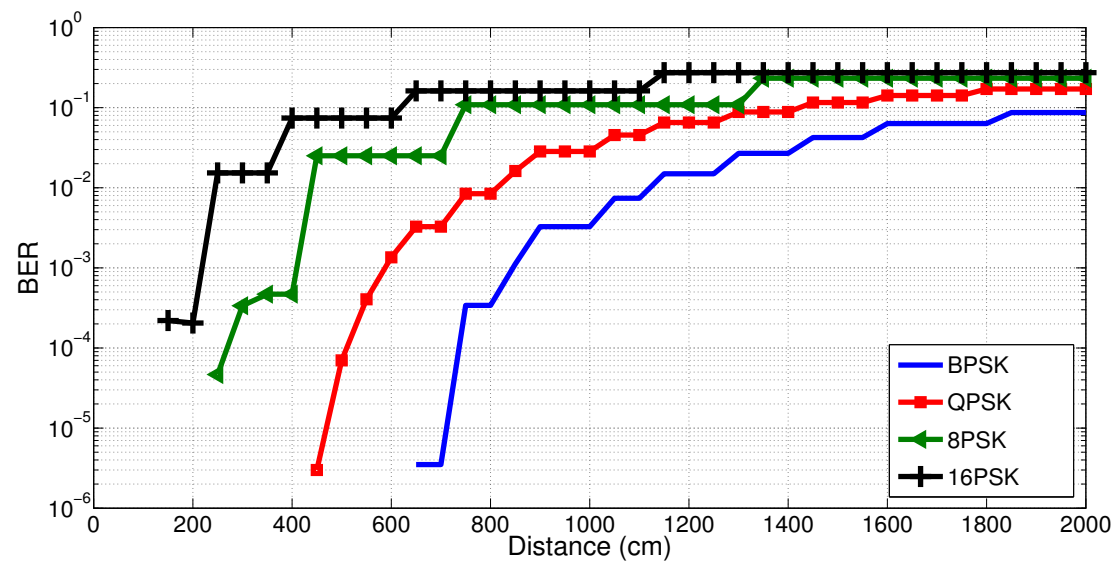

Figure 5.8: Bit error rate vs distance $(\mathrm{cm})$ for modulation methods using channel B for transmission.

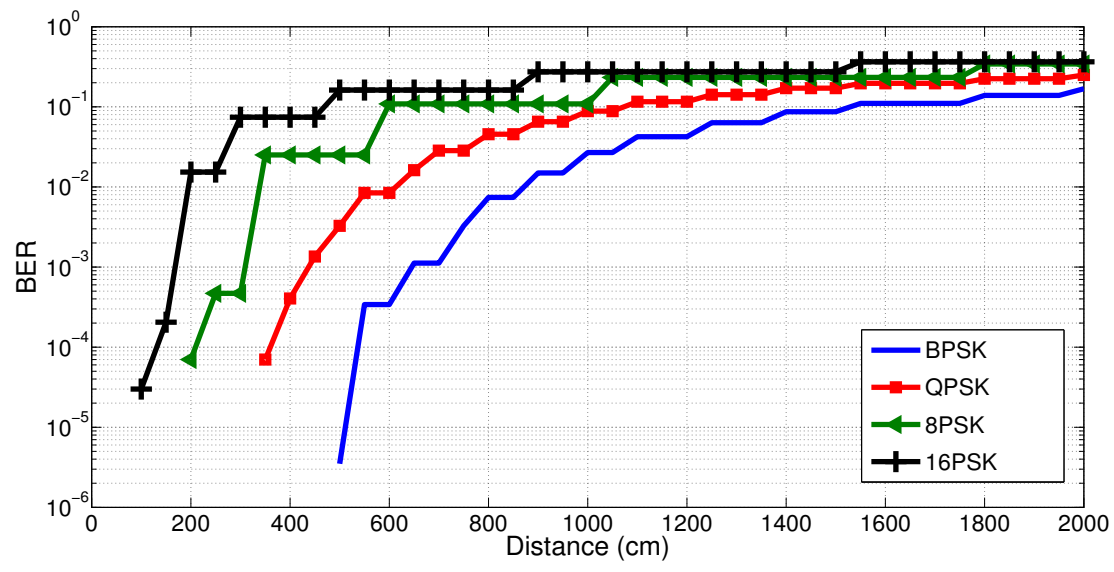

Figure 5.9: Bit error rate vs distance $(\mathrm{cm})$ for modulation methods using channel $\mathrm{C}$ for transmission.

Having the BER vs distance corresponding to each channel, we calculate data rate vs distance. Figures 5.10-5.12 show the result when TAP uses channel A, B, or C, respectively. 


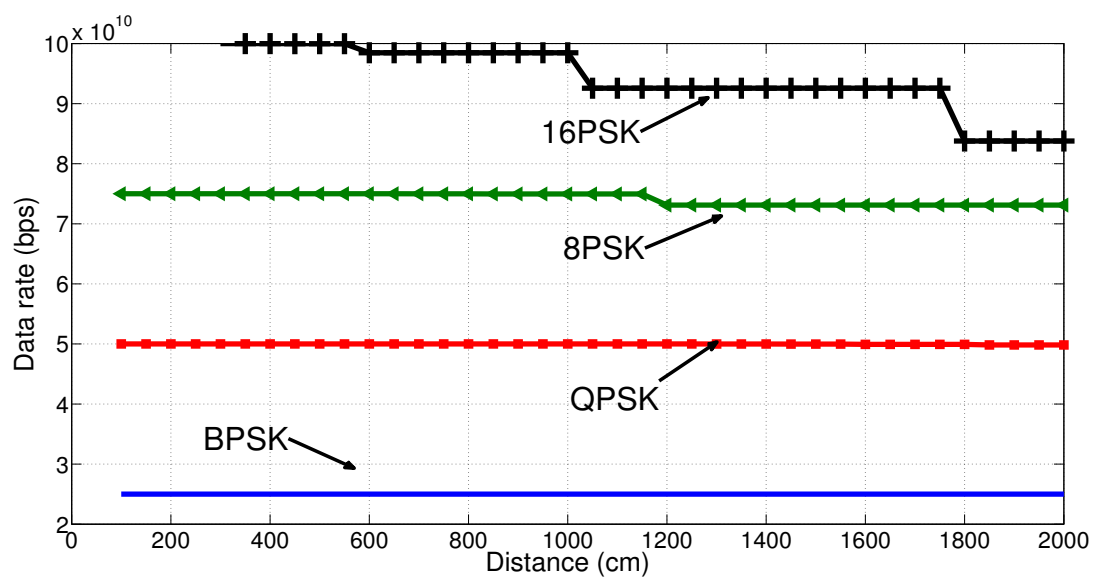

Figure 5.10: Data rate (bps) vs distance $(\mathrm{cm})$ for modulation methods using channel A.

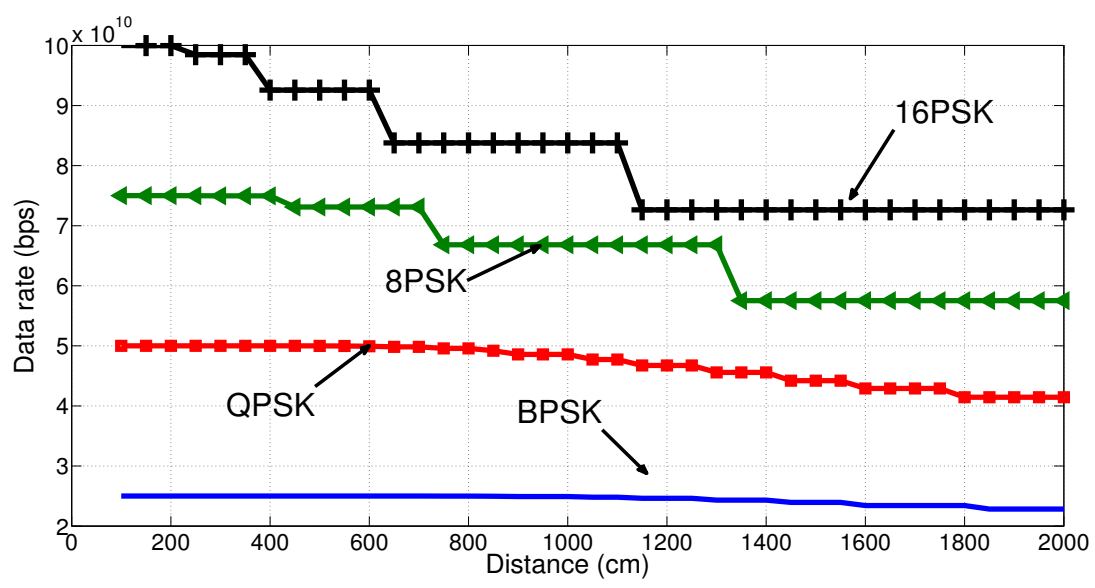

Figure 5.11: Data rate (bps) vs distance $(\mathrm{cm})$ for modulation methods using channel B.

\subsubsection{SCA Simulation Results}

For simulation, we assume there are 10 users at different distances. Based on the result from Figure 5.7, when TAP uses channel A, it can send data to users with distances up to $2000 \mathrm{~cm}$ with $\mathrm{BER}<\mathrm{BER}_{T h}$. Similarly, from the result of Figure 5.8, when TAP uses channel B, it covers users with distances up to $700 \mathrm{~cm}$. Finally, the result from Figure 5.9 shows that TAP can cover users with distances up to $500 \mathrm{~cm}$ using channel C. 


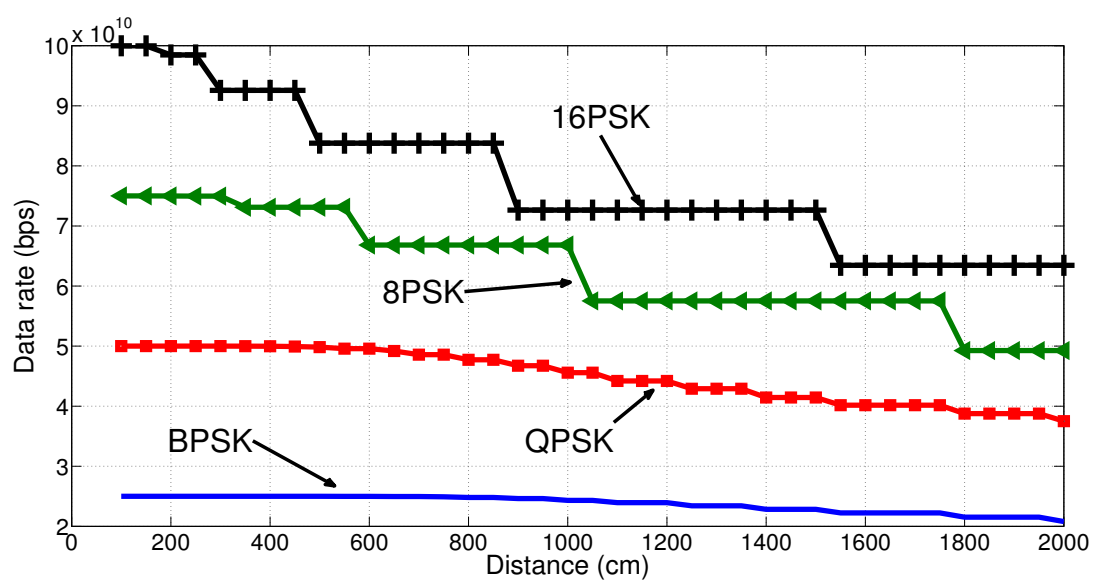

Figure 5.12: Data rate (bps) vs distance $(\mathrm{cm})$ for modulation methods using channel C.

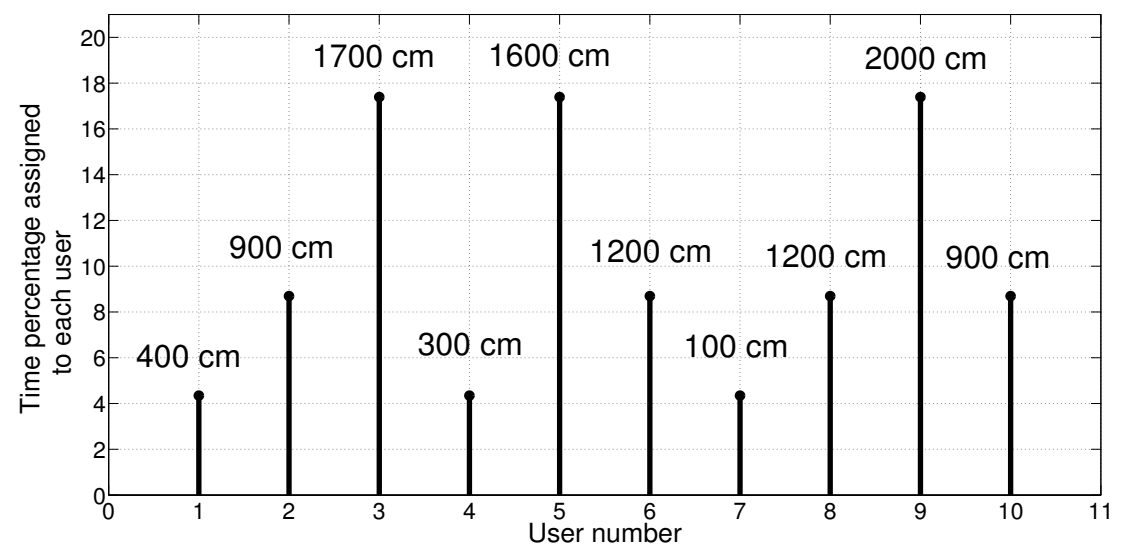

Figure 5.13: The assigned time percentage to each user when channel $\mathrm{A}$ is used.

To maintain fairness between the users, as is explained in section 5.2, TAP assigns a time slot to each user such that all users achieve the same data rate. We set $T_{\min }$ to be $600 \mu \mathrm{s}$ and based on that we assign the proper time slot to users.

We assign TAP-user distances of up to $2000 \mathrm{~cm}, 700 \mathrm{~cm}$, and $500 \mathrm{~cm}$ when TAP uses channel A, B, or C, respectively. Figures 5.13-5.15 show the percentage of time that TAP assigns to users corresponding to each scenario.

Figure 5.16 shows the data rate that each user achieves, from equations (5.2) 


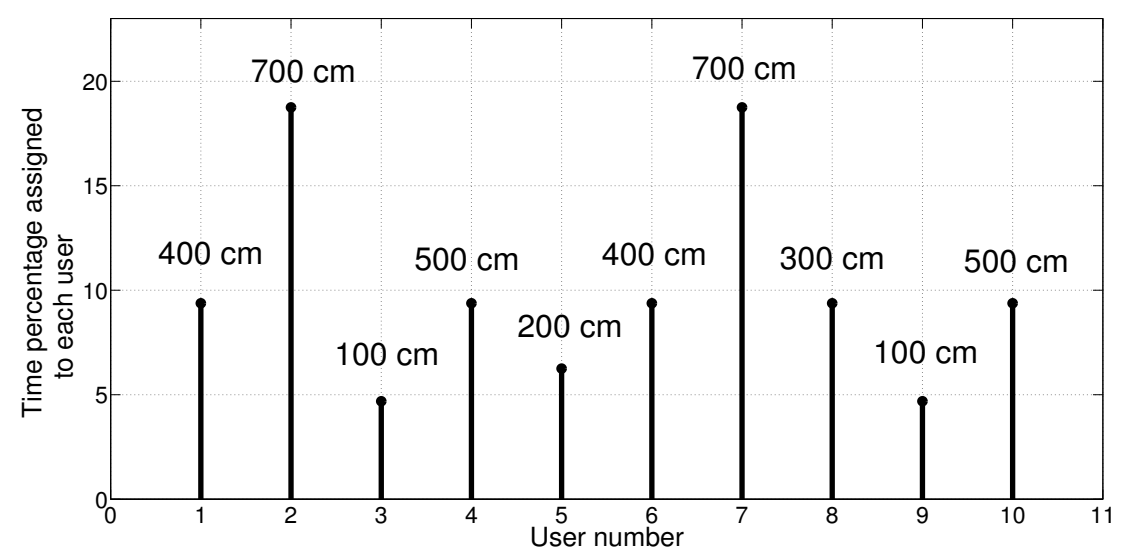

Figure 5.14: The assigned time percentage to each user when channel B is used.

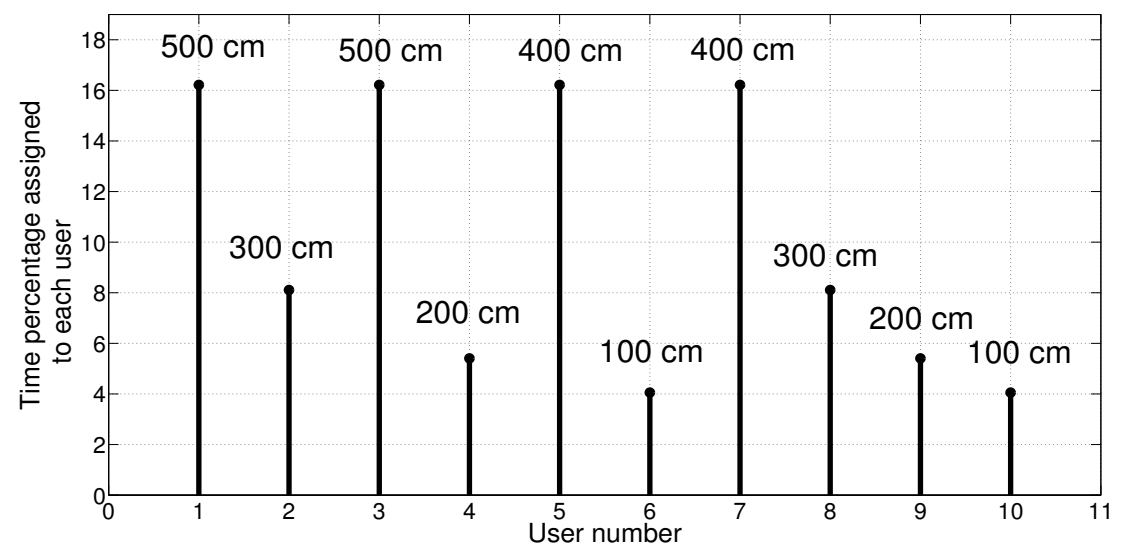

Figure 5.15: The assigned time percentage to each user when channel $\mathrm{C}$ is used.

and (5.3), when TAP uses channel A, B, or C. As is obvious, in all cases the fairness is maintained and all of the users achieve the same data rate. We should note that the achieved data rate for each channel depends on the average TAP-user distances. 


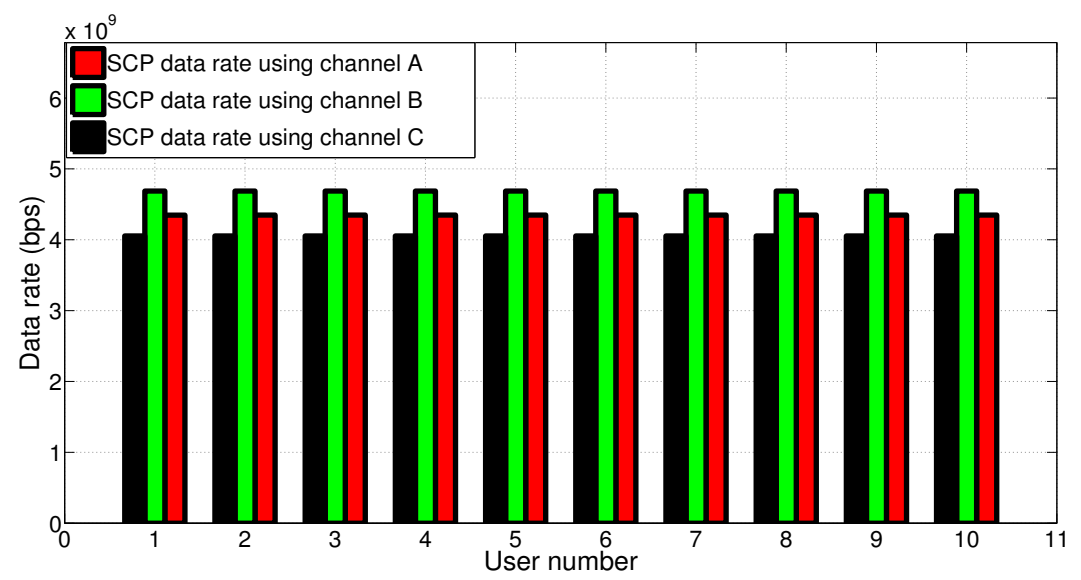

Figure 5.16: SCA downlink data rate when TAP uses channel A, B, or C.

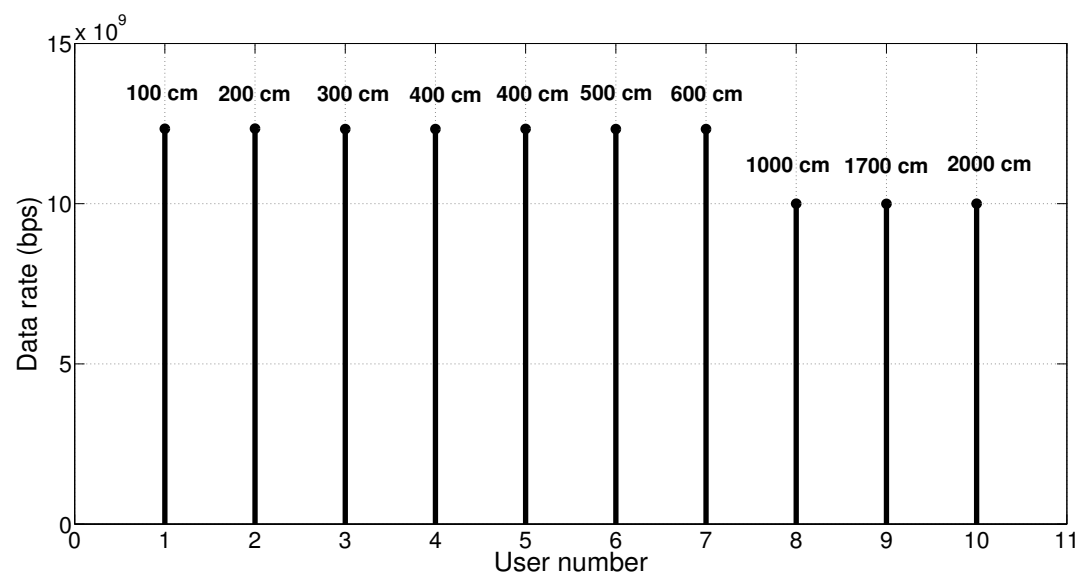

Figure 5.17: MCA downlink data rate.

\subsubsection{MCA Simulation Results}

In this section we show the simulation result when using MCA. Again, we assume that we have 10 users and randomly assign TAP-user distances up to $2000 \mathrm{~cm}$ to them. We consider the time slot duration, $T$, of $200 \mu \mathrm{s}$. We use the MCA and run the program for 5000 time slots.

From equations (5.4) and (5.5) we calculate the data rate for each user. Figure 


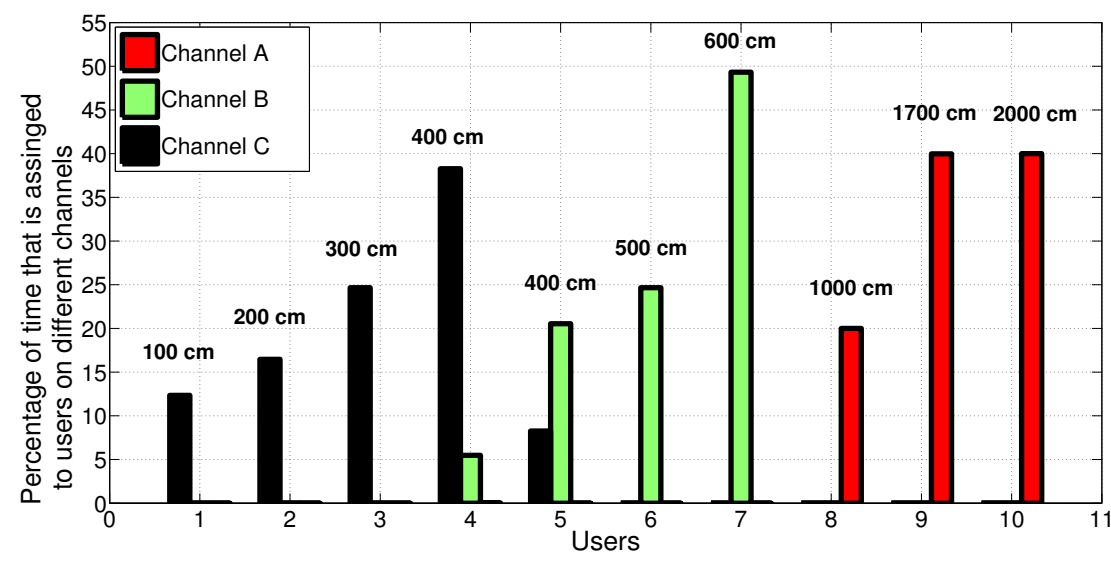

Figure 5.18: The percentage of time that TAP assigns different channels to each user in MCA.

5.17 shows the achieved data rate for each user and Figure 5.18 shows the percentage of time that TAP assigns to users on each channel. As we see, users 8-10 achieve lower data rate in comparison to other users. That is, because of the long TAP-user distances, only channel A can cover these users with a low modulation order. Hence, TAP uses channel A only for these three users and their achieved data rate could not go any higher. At the same time TAP uses channel B to send data to users $4-7$ and channel $\mathrm{C}$ for users $1-5$.

\section{THE EFFECT OF TRANSMITTED POWER ON TRANSMITTER- RECEIVER DISTANCE}

In the simulations we assume that the transmitted signal power is $37 \mathrm{~mW}$ and we show that on channel $\mathrm{A}$, receivers up to 20 meters are able to receive the signal using BPSK modulation.

As the $\mathrm{THz}$ technology is improved every year and newer techniques emerge, 


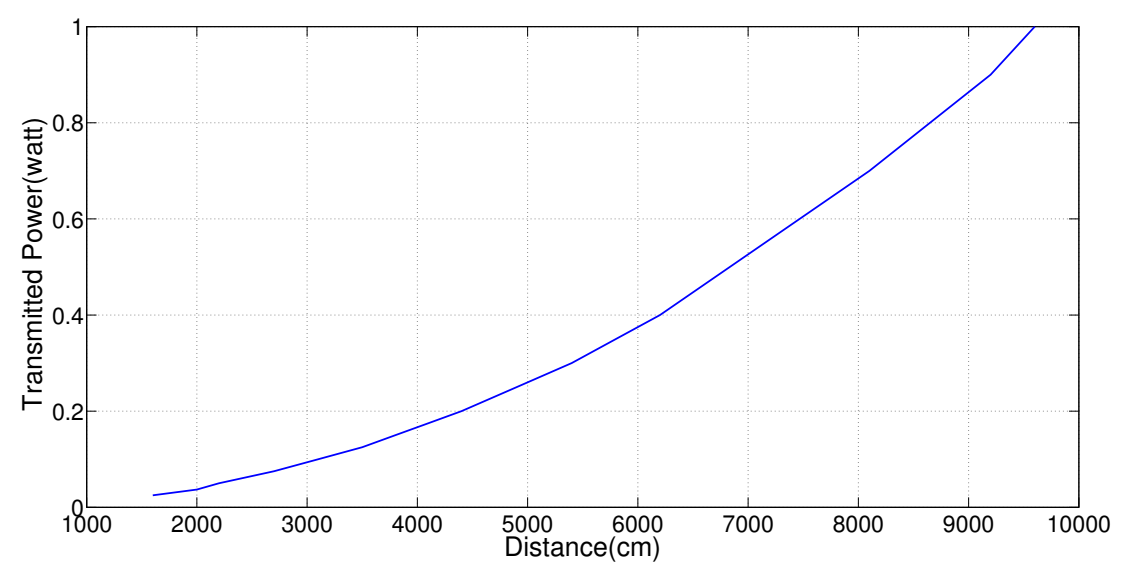

Figure 5.19: Transmitted power vs. coverable transmitter-receiver distance when BPSK is used on channel $\mathrm{A}$ and $\mathrm{BER} \leq 10^{-4}$.

it is predicted that in near future newer $\mathrm{THz}$ hardware will have higher transmitted power than what is available today. With higher transmitted power longer transmitter-receiver distances can be covered as well if a receiver is using BPSK modulation on channel A with $\mathrm{BER} \leq 10^{-4}$. Figure 5.19 shows power in watt vs transmitter-receiver distance in $\mathrm{cm}$. As is shown, increasing the transmitted power increases the covered transmitter-receiver distance and distances up to $96 \mathrm{~m}$ can be covered with transmitted power of 1 watt.

\section{SUMMARY}

In this chapter we derived an omni-directional $\mathrm{THz}$ channel model. We investigated the modulation of CW signals and provided two rate adaptation algorithms. Simulation results showed that all the users with different transmitter-receiver distances will achieve almost the same data rate in our channel and fairness is maintained. Assuming the ability of increasing the transmitted power in the near future, we provided a study on the effect of transmitted power on the longest 
transmitter-receiver distance that can be covered in our channel. 
Chapter 6

LITERATURE REVIEW

\section{LITERATURE REVIEW ON TERAHERTZ GENERATION AND DETECTION TECHNIQUES}

There are different techniques to generate and detect $\mathrm{THz}$ signals. In the following subsections first we will summarize $\mathrm{THz}$ generation techniques and then we will explain the detection methods. After that we will discuss the recently achieved data rates using those techniques.

\subsection{1 $\mathrm{THz}$ Generation Techniques}

$\mathrm{THz}$ signal generation techniques are mainly categorized as either photonic or all electronic techniques. The photonic technique is further divided into optoelectronic and quantum cascade lasers (QCLs) [71].

\section{Photonic Techniques}

Optoelcetronic Technique Optoelectronic is one of the most widely-used techniques in $\mathrm{THz}$ communications. In this technique, frequencies from optical to near-IR are down-converted to $\mathrm{THz}$ frequencies. Researchers use optoelectronic technique to generate both THz time-domain signals (THz-TDS), which is also called pulsed signals, and continuous-wave $(\mathrm{CW})$ signals.

In 1989, Exter et al. [14] developed a technique to generate THz-TDS (pulsed signals). Each of these pulses is a time-domain wave form of the electric field of 
the $\mathrm{THz}$ electromagnetic wave [41] and lasts only a few picoseconds. As a result, each pulse contains the information of both amplitude and phase of the electric field and the whole frequency range up to $\sim 3 \mathrm{THz}$.

In THz-TDS technique, transmitters and receivers use photoconductive dipole antenna (PDA) structures. PDAs have fast photoconductive material, such as gallium arsenide (GaAs), that acts as a fast switch. A short optical signal from a femto-second laser, for example a mode-locked Ti:sapphire laser, illuminates the GaAs, and the photoconductive switch closes. This process generates the ultrashort signal. Then, an electro-optic (EO) or electro-absorption (EA) modulator modulates the optical signal. The optical signal is then converted into an electrical signal by a photodiode (PD) or a photoconductor (PC) and then an antenna emits the generated electrical signal into the air.

In general, generating a pulsed $\mathrm{THz}$ signal with a higher power would result in a signal with lower bandwidth and generating a signal with a bandwidth larger than 1 $\mathrm{THz}$ results in a lower transmit power. The company T-Ray Science demonstrated $55 \mu \mathrm{W}$ of $\mathrm{THz}$ power for $100 \mathrm{~mW}$ of optical input power [16].

The THz-TDS technique is used widely for weapons detection, imaging of objects, and spectroscopy of gas, liquid and solid materials [44, 74]. The data rate that can be achieved with this technique is limited by the repetition rate of the Ti:sapphire laser, which is about $107 \mathrm{GHz}$ [40]. Figure 6.1 is a block diagram of the optoelectronic technique that generates THz-TDS. Here, with Fourier transform relations, the frequency characteristics of the time-domain signal can be found.

Besides THz-TDS systems, optoelectronic techniques can be used to generate continuous wave (CW) signals. Verghese et al. [65] developed this method in 1998. For generating CW signals, photo-mixing (heterodyning) technique is used to generate a narrowband $\mathrm{THz}$ signal. In photo-mixing technique, two collinear optical 


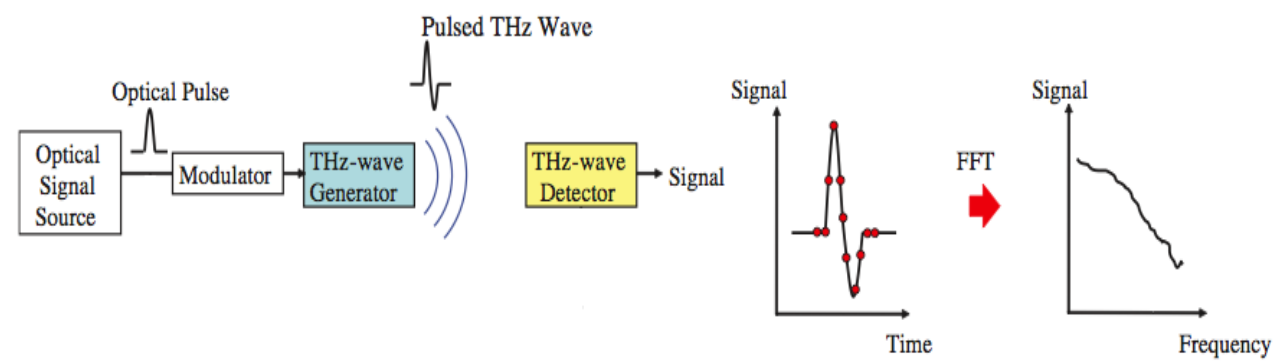

Figure 6.1: Block diagram of optoelectronic technique that generates THz-TDS [44]

to near-IR signals illuminate a PDA and generate a $\mathrm{THz}$ signal at the difference frequency of the two signals. To have efficient photo-mixing, the polarizations, frequencies, and phases of the input optical signals must be stable. Nagatsuma et al. [44] reported on generating the output power of $400 \mu \mathrm{W}(-40 \mathrm{dBm})$ in generating CW signals with optoelectronic sources.

Figure 6.2 is a block diagram of optoelectronic technique that generates CW signals. As the figure shows, the generated signal occupies only a narrow band of the frequency domain.
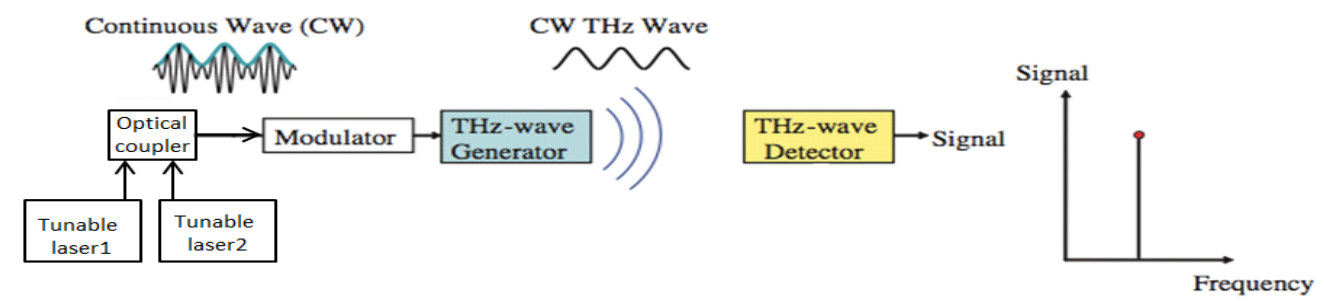

Figure 6.2: Block diagram of optoelectronic technique that generates $\mathrm{CW}$ signal with two tunable lasers. 
Besides PDA, UTC-PD (uni-traveling-carrier photodiode) can be used in generation and detection of $\mathrm{THz}$ signals in both pulsed and $\mathrm{CW}$ based systems. The PDA can generate signals with relatively larger bandwidth whereas UTC-PD can generate signals with higher $\mathrm{THz}$ power [71].

Figure 6.3 compares the transmitted signal's power of the THz-TDS system and the heterodyne based system that generates CW signals. As the result show, with the same input power, the output power generated in CW technique is lower than the output power generated in pulsed technique.

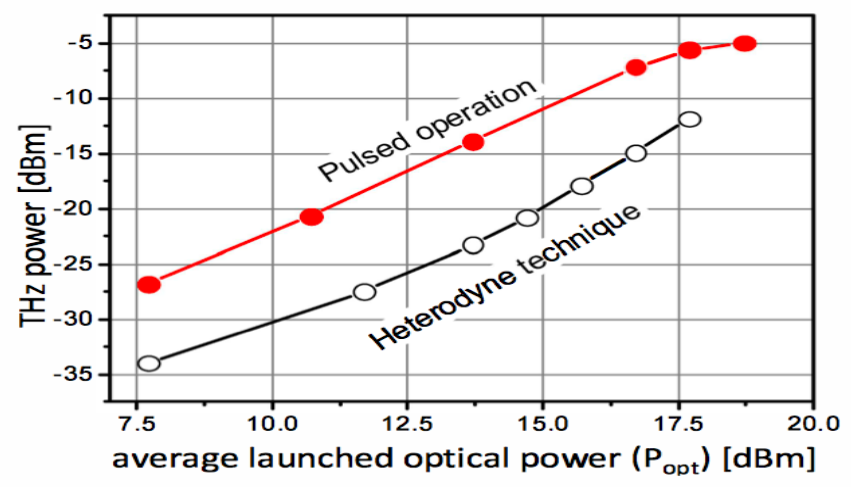

Figure 6.3: A power comparison between the signals generated based on THz-TDS and Heterodyne systems [40].

Quantum Cascade Laser (QCL) In this technique, tunable frequency $\mathrm{THz}$ semiconductor lasers are used as QCLs to generate pulsed and CW THz signals [71]. A QCL emits photons due to unipolar intersubband transitions in the quantum wells (QWs) of a semiconductor heterostructure [36].

The first QCL was demonstrated in 1994 by Bell Labs that could generate signals with $75 \mathrm{THz}$ frequency [15]. In 2001, Kohler et al. developed a QCL to generate 4.4 THz signals. Today QCLs cover the range of 1.2-5.0 THz. [35, 36]. 
However, very low temperatures $(<40 \mathrm{~K})$ are required for QCLs to work and systems that can operate below $1.5 \mathrm{THz}$ frequency at room temperature won't be available in the near future. This requirement of the low temperature, limits the usage of QCLs and makes this technique less practical [16, 68].

\section{Electronic Technique}

While optoelectronic based generators down-convert the high frequency of optical signals to the $\mathrm{THz}$ frequency, electronic based generators up-convert the lower frequency of radio frequency $(\mathrm{RF})$ signals to $\mathrm{THz}$ frequency. For that, a series of frequency doublers and triplers, such as Schotthky diode (SBD) multipliers, are used $[16,71]$.

Fig. 6.4 shows a microwave frequency multiplier that generates $\mathrm{THz}$ signals. A 16.66 GHz source signal is up-converted by a frequency tripler, then the signal is amplified, and then is tripled again to generate a $150 \mathrm{GHz}$ signal. The generated signal is then combined with a local oscillator (LO) from a dc-10 GHz signal generator in a mixer to generate a signal with the center frequency of $300 \mathrm{GHz}$ $[16,27]$.

\section{Conclusion}

Microwave multiplier systems can provide milliWatts $(\mathrm{mW})$ of $\mathrm{THz}$ power with several Gigahertz of bandwidth. On the other hand optoelectronic systems can generate a signal with a larger bandwidth, but they are more expensive than microwave based systems and their output power is in the order of $\mu \mathrm{W}[16]$.

Due to the limited maximum frequency of oscillation, electronic based systems have not been considered seriously for generating $\mathrm{THz}$ signal for relatively high frequencies. However, Huang et al. anticipate that signals beyond $2 \mathrm{THz}$ can be 


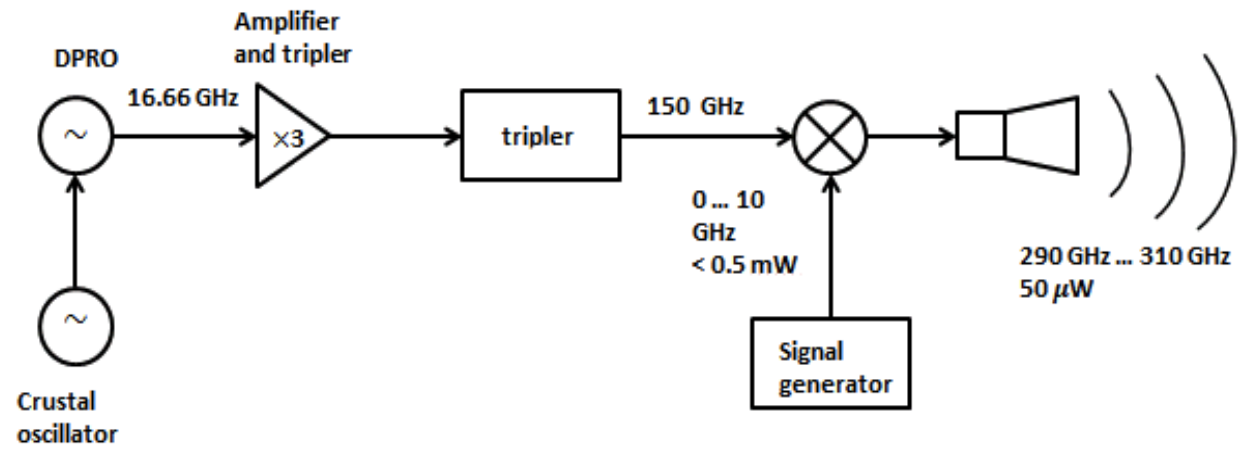

Figure 6.4: A transmitter with microwave frequency multiplier components [27].

generated using all electronic systems with output power up to $-1.5 \mathrm{dBm}(700 \mu \mathrm{W})$ $[25]$.

\subsubsection{THz Detection Techniques}

$\mathrm{THz}$ detection is categorized into homodyne, heterodyne, direct direction, and optoelectronics techniques. Optoelectronics is further divided into electro-optic sampling and photoconductive sampling methods.

\section{Homodyne Detection}

Systems based on homodyne detection are built using broadband thermal detectors such as Golay cell and bolometers. Here LO, which has the same frequency as the generated $\mathrm{THz}$ signal at the transmitter, is mixed with the received signal. In this technique, there is no need to down-convert the received signal to an intermediate frequency (IF). Here a direct conversion to the baseband is implemented and as a result the phase and the amplitude information of the received $\mathrm{THz}$ signal are derived [30, 33, 71]. 


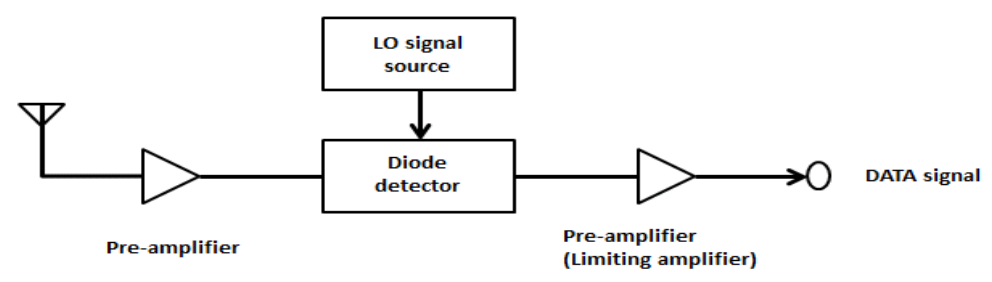

Figure 6.5: Typical configuration of a receiver based on heterodyne technique [43].

\section{Heterodyne Detection}

As in homodyne detection, a radiation of a $\mathrm{LO}$ is used at the receiver to detect the received signal. However, in the heterodyne technique, LO does not have the same frequency as the received $\mathrm{THz}$ signal.

Here, the received signal and the LO are superimposed at a mixer to downconvert the received THz signal to a relatively low frequency signal. As a result a non-zero IF is generated. In this technique, a mixer that has a strong electric field quadratic nonlinearity, such as SBD mixer, is used. The following electrical circuits can then retrieve the phase and the amplitude of the received THz signal.

Recently Monolithic microwave integrated circuits (MMIC) have been developed in heterodyne detection technique. These curcuits have small dimensions and can be used to mix and detect the received $\mathrm{THz}$ signal with frequency less than $500 \mathrm{GHz}[30,43,51,71]$. Fig. 6.5 shows the block diagram of a receiver that uses heterodyne technique to detect the received $\mathrm{THz}$ signal.

\section{Direct Detection}

In this technique, focusing optic elements, such as lenses and mirrors, are used to collect the received signal at the receiver and send it to the detector. It is possible 


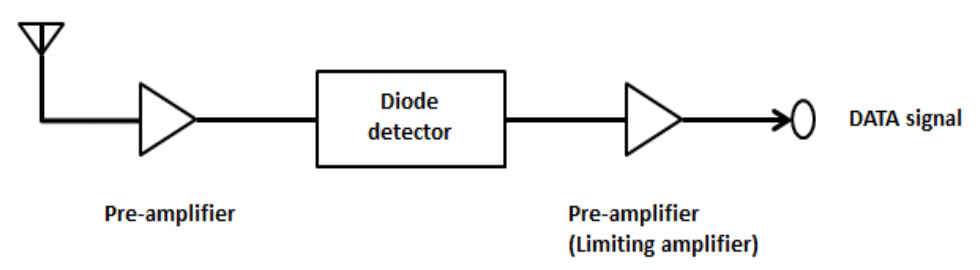

Figure 6.6: Typical configuration of a receiver based on direct detection technique [43].

to put an optical filter before the detector to remove background noise with wavelengths other than the detected signal. An amplifier amplifies the electrical signal from the detector and the signal is then further processed. In direct direction, only the amplitude of the received signal can be measured. Therefore, this detection technique only works with amplitude based modulations such as amplitude shift keying (ASK) and on-off keying (OOK). Direct detection is easy to do with a diode detector such as a SBD with a cutoff frequency of 1-10 THz [43, 51]. Figure 6.6 shows a receiver that uses direct detection technique to detect the received signal.

\section{Optoelectronic Detection}

Electro-optic sampling In electro-optic sampling, a probe optical pulse and the received $\mathrm{THz}$ signal are sent to a sensor crystal and passed through it. As a result of the nonlinear property of the crystal, the $\mathrm{THz}$ signal modulates the polarization ellipticity of the probe pulse. Therefore, the amplitude and phase information of the $\mathrm{THz}$ signal can be analyzed based on the polarization of the probe pulse [71]. 
Photoconductive sampling Photoconductive sampling is widely used in THz-TDS systems. In this technique wide-band PDAs are used. Here, ultrafast optical pulses are applied as sampling gates. The interaction between the received $\mathrm{THz}$ signal and the sampling pulses in PDA will generate a photocurrent that is proportional to the incident $\mathrm{THz}$ electric field [54].

In synchronous photoconductive sampling, the sampling gate comes from the same pulse source as the $\mathrm{THz}$ signal. Here the temporal profile of the received $\mathrm{THz}$ pulse is obtained by scanning the relative delay between the $\mathrm{THz}$ signal and the probe pulse. One disadvantage of this method is a long measurement time due to the mechanical delay stage [41]. In asynchronous photoconductive sampling (AOS), the sampling gate comes from an independent pulse source to decrease the measurement time and make the process faster and avoid the mechanical delay. Here two femtosecond lasers are used; one of them is used to produce the $\mathrm{THz}$ pulses at the transmitter and the other one samples the produced pulses [8].

Furuaya et al. [20] proposed a new sampling technique. They used only one femtosecond laser with a repetition frequency that is continuously changed. As a result, their system is cheaper but as fast as AOS technique.

Kim and Yee [31] proposed a sampling technique in which they used two synchronized Ti:sapphire femtosecond lasers with center wavelength of $800 \mathrm{~nm}$ and pulse duration of 10 and $20 \mathrm{fs}$, respectively with a repetition rate of $100 \mathrm{MHz}$. They used electronically controlled optical sampling and achieved a system that has a 50 times higher measurement speed than the AOS system.

\section{Conclusion}

Using electronic techniques, we can achieve higher output power than optoelectronic techniques. However, with today's available technology, only generating 
and detecting narrow band signals with relatively low center frequency is possible using electronic based transmitters and receivers [30, 71] and generating wide band or pulsed $\mathrm{THz}$ signals requires photonics techniques [33, 43].

The proper $\mathrm{THz}$ detection technique usually, but not always, depends on the $\mathrm{THz}$ generation technique. For example, systems that use microwave frequency multipliers in their transmitters would use the same or similar microwave sources at the receiver - to down-convert and detect the received signal. Systems that have optoelectronic transmitters would use the same principle to detect the received signal — as in THz time-domain or CW photomixing [16].

With the advent of new technologies $\mathrm{THz}$ transmitters and receivers are getting cheaper, smaller, faster, and more practical. Therefore, more researchers are starting to use THz techniques to send data. For example, recent works on CMOS technology and single chip design in Terahertz transmitters [18, 25] and receivers [57] indicates that $\mathrm{THz}$ transmitters and receivers are getting smaller and cheaper in both electronic and optoelectronic based systems. 


\section{LITERATURE REVIEW ON ACHIEVED DATA RATES}

THz-TDS systems have been used in applications such as Terahertz imaging [17], biological spectroscopy [70], and communication in nano-networks [29]. However, to the best of our knowledge, the pulsed approach has not been used in Terahertz communication systems over a distance of longer than few millimeters, as in nano-networks. To date all communication systems have been highly directional CW systems and mostly use simple modulation methods such as OOK and ASK. For example, Song et al. [60] used $0.25 \mathrm{THz}$ carrier frequency and transmitted $8 \mathrm{Gbit} / \mathrm{s}$ error free data over $50 \mathrm{~cm}$ distance with ASK modulation. They used optoelectronic technique in designing their transmitter that had two tunable laser sources (TLSs) with a frequency difference of $0.25 \mathrm{THz}$ and a UTC-PD module with a horn antenna. They used direct detection technique with an SBD at the receiver. The output power of their system was $\sim 200 \mu \mathrm{W}$. They also used a dielectric lens after the horn antenna to increase the directivity of the radiated signal. The bandwidth that they used was $4.5 \mathrm{GHz}\left(4.5 \times 10^{-3} \mathrm{THz}\right)$.

Hirata et al. used $0.12 \mathrm{THz}$ carrier frequency with optoelectronic based transmitter with a UTC-PD and an electronic based receiver with an SBD for direct detection [23]. Later they replaced all the photonics components by electronic components with the high transmitter power of $16 \mathrm{dBm}(39.8 \mathrm{~mW})$. They were able to achieve $10 \mathrm{Gbit} / \mathrm{s}$ data transmission rate with ASK modulation and forward error correction technique over a distance of $5.8 \mathrm{~km}[22]$.

Kallfass et al. [30] chose $0.22 \mathrm{THz}$ carrier frequency with OOK modulation. The authors used electronic techniques with microwave frequency multipliers and achieved data rates up to $40 \mathrm{Gbit} / \mathrm{s}$ over a $50 \mathrm{~cm}$ distance. However, they only got low BER for data rates up to 15 Gbps while for higher data rates they were 
not able to get a good signal quality at the receiver.

Antes et al. [6] presented an electronic based transmitter with microwave multipliers at the receiver that utilizes $0.24 \mathrm{THz}$ frequency over a $60 \mathrm{~cm}$ distance with OOK modulation that resulted in a 25 Gbps data rate.

Zhang et al. [73] proposed a switch-based ASK modulator for an electronic based system. With this proposed method, the authors were able to achieve 10 Gbit/s data rate with $0.135 \mathrm{THz}$ carrier frequency over $20 \mathrm{~cm}$ distance.

Song et al. [61] considered data transmission on the $0.3 \mathrm{THz}$ carrier frequency over a 50-cm distance. They used UTC-PD in their optoelectronic transmitter and SBD based direct detection technique in their receiver. With an output power of less than $200 \mu \mathrm{W}$, the authors got a BER of $1 \times 10^{-10}$. They achieved an error free data rate of as high as $24 \mathrm{Gbit} / \mathrm{s}$ with ASK modulation.

Ducournau et al. [13] used optoelectronic techniques with output power of $1 \mu \mathrm{W}$ at the transmitter and electronic techniques at the receiver with a $0.4 \mathrm{THz}$ carrier frequency. They achieved 40 Gbps data rate with ASK modulation.

Besides ASK and OOK, duobinary baseband modulation has also being used for data transmission. Researchers in Bell Laboratories [39] used a higher carrier frequency of $0.625 \mathrm{THz}$. They used a commercially available transmitter to generate CW signals. This electronic based transmitter had microwave frequency multipliers with an output power of $1 \mathrm{~mW}$. In this system, 4 frequency doublers followed by a frequency tripler up-converted the $0.013 \mathrm{THz}$ input signal to $0.625 \mathrm{THz}$. At the receiver an SBD converts the THz signal into baseband. Researchers achieved $2.5 \mathrm{Gbit} / \mathrm{s}$ data rate over a few-meters distance $(<10 \mathrm{~m})$ between transmitter and receiver.

Recently some researchers have utilized higher order modulation to achieve better spectrum efficiency. For example, Wang et al. [67] transmitted data over 
$1.5 \mathrm{~km}$ distance between transmitter and receiver. They used electronic based transmitter with microwave frequency multipliers. The authors achieved $10 \mathrm{Gbit} / \mathrm{s}$ over a non-real-time channel with software modulator and 2 Gbit/s over a real-time channel with hardware modulator using 16QAM modulation.

Lu et al. [38] demonstrated a system at $0.34 \mathrm{THz}$ band with all electronic components. They used 16QAM modulation and transmitted 3Gbps data over a $30 \mathrm{~cm}$ distance.

Koenig et al. [34] for the first time achieved a data rate as high as $100 \mathrm{Gbit} / \mathrm{s}$ over a 20 meter distance between transmitter and receiver. The carrier frequency was $0.237 \mathrm{THz}$ with a bandwidth of $0.035 \mathrm{THz}$. They utilized 8QAM and 16QAM modulation methods and designed relatively small optoelectronic based transmitter and electronic based receivers. Having small sizes, these transmitter and receiver systems may enable $\mathrm{THz}$ communication in future smartphones and tablets.

Considering the $\mathrm{THz}$ channel attenuation, Han and Akyildiz [21] proposed a distance adaptive modulation method. In this method, based on transmitterreceiver distance, transmission windows with path loss of less than $160 \mathrm{~dB}$ are selected. These transmission windows are divided into smaller windows that are used to transmit multi-carrier signals. In this method, the assigned bandwidth and modulation order to each user depends on the transmitter-receiver distance. This method however suffers from the complexity of the control channel unit and channel feedback path. Also, the transmitter uses thousands of carriers to send data to a receiver. Having that many carriers at once requires a complex transmitter and receiver which is not feasible today.

Table 6.1 is a summary of the work discussed above in addition to some other related work. 
Table 6.1: A summary of related works. CMOS: complementary metal oxide semiconductor, MMIC: monolithic microwave integrated circuits, SBD: schottky barrier diode.

\begin{tabular}{|c|c|c|c|c|c|c|}
\hline $\begin{array}{c}\text { Frequency } \\
(\mathrm{THz})\end{array}$ & $\begin{array}{c}\text { Data Rate } \\
\text { (Gbit/s) }\end{array}$ & $\begin{array}{l}\text { Distance } \\
\quad(\mathrm{m})\end{array}$ & Modulation & $\begin{array}{c}\text { transmitter } \\
\text { Technique }\end{array}$ & $\begin{array}{l}\text { receiver } \\
\text { Technique }\end{array}$ & Ref \\
\hline 0.125 & 10 & 200 & ASK & optoelectronic & $\begin{array}{c}\text { heterodyning } \\
\text { (MMIC) }\end{array}$ & [24] \\
\hline 0.25 & 8 & 0.5 & ASK & optoelectronic & $\begin{array}{l}\text { direct detection } \\
\quad(\mathrm{SBD})\end{array}$ & {$[60]$} \\
\hline 0.2 & 1 & 2.6 & ASK & optoelectronic & $\begin{array}{l}\text { heterodyning } \\
\text { (mixer) }\end{array}$ & {$[12]$} \\
\hline 0.12 & 10 & 5800 & ASK & electronic & direct detection & {$[22]$} \\
\hline 0.3 & 0.096 & 0.7 & 64QAM & electronic & $\begin{array}{l}\text { heterodyning } \\
\text { (mixer) }\end{array}$ & [28] \\
\hline 0.625 & 2.5 & $<10$ & Duobinary & electronic & $\begin{array}{l}\text { direct detection } \\
\quad(\mathrm{SBD})\end{array}$ & [39] \\
\hline 0.22 & $15-40$ & 10 & OOK & electronic & $\begin{array}{l}\text { heterodyning } \\
\text { (MMIC) }\end{array}$ & {$[30]$} \\
\hline 0.24 & 25 & 60 & OOK & electronic & $\begin{array}{l}\text { homodyning } \\
\text { (MMIC) }\end{array}$ & {$[6]$} \\
\hline 0.0875 & 100 & 1.2 & 16QAM & optoelectronic & $\begin{array}{l}\text { heterodyning } \\
\text { (SBD) }\end{array}$ & {$[47]$} \\
\hline 0.135 & 10 & 0.2 & ASK & electronic & $\begin{array}{l}\text { heterodyning } \\
\text { (CMOS) }\end{array}$ & {$[73]$} \\
\hline 0.3 & 24 & 0.5 & ASK & optoelectronic & $\begin{array}{l}\text { direct detection } \\
\quad(\mathrm{SBD})\end{array}$ & {$[61]$} \\
\hline 0.146 & 1 & 0.025 & OOK & optoelectronic & $\begin{array}{l}\text { heterodyning } \\
\text { (SBD) }\end{array}$ & [19] \\
\hline 0.22 & 30 & 20 & ASK & electronic & $\begin{array}{l}\text { homodyning } \\
\text { (MMIC) }\end{array}$ & {$[5]$} \\
\hline 0.542 & 2 & 0.01 & ASK & electronic & $\begin{array}{l}\text { direct detection } \\
\quad(\mathrm{SBD})\end{array}$ & {$[26]$} \\
\hline 0.14 & 10 & 1500 & 16QAM & electronic & $\begin{array}{l}\text { heterodyning } \\
\text { (SBD) }\end{array}$ & {$[67]$} \\
\hline 0.24 & 30 & 40 & 8PSK & electronic & $\begin{array}{l}\text { heterodyning } \\
\text { (MMIC) }\end{array}$ & {$[4]$} \\
\hline 0.196 & 0.1 & 0.5 & QPSK & electronic & $\begin{array}{l}\text { heterodyning } \\
\text { (MMIC) }\end{array}$ & {$[55]$} \\
\hline 0.34 & 3 & 0.3 & 16QAM & electronic & $\begin{array}{l}\text { heterodyning } \\
\text { (MMIC) }\end{array}$ & [38] \\
\hline 0.3 & 24 & 0.3 & ASK & optoelectronic & $\begin{array}{l}\text { heterodyning } \\
\text { (MMIC) }\end{array}$ & {$[62]$} \\
\hline 0.3 & 48 & 1 & OOK & optoelectronic & $\begin{array}{l}\text { direct detection } \\
\quad(\mathrm{SBD})\end{array}$ & {$[43]$} \\
\hline 0.237 & 100 & 20 & 16QAM & optoelectronic & $\begin{array}{l}\text { heterodyning } \\
\text { (MMIC) }\end{array}$ & {$[34]$} \\
\hline
\end{tabular}




\section{Chapter 7}

\section{CONCLUSION}

In this dissertation we studied Terahertz communication techniques to provide tens of Gigabits/second data rates to users. The following are the main contributions of this research:

1. We developed detailed channel characterization for directional and omnidirectional channels:

- We conducted experimental measurements using Picometrix T-Ray 4000 system under different channel settings. The results of channel measurements show that pulsed Terahertz signals are appropriate to be used in directional channels whereas continuous wave signals can be used in non-directional channels as well.

- We modeled channel transfer functions for directional and omni-directional channels to be used in our simulations and to be provided to the general research community. In these models, we investigated the effect of atmospheric attenuation corresponding to oxygen, water vapor, nitrogen, and carbon dioxide for the frequency range of 0-2 Terahertz. We compared the results of our experimental measurements with the developed theoretical models and showed that they are in a close agreement.

2. We analyzed pulsed Terahertz signals in wireless communications for the first time in channels other than nano-networks: 
- Pulsed Terahertz signals are used widely for purposes other than communications, such as imaging of objects, weapons detection, and spectroscopy of gas, liquid and solid materials. However, we used them in communication systems with transmitter-receiver distances of few meters and achieved Terabits/second data rates.

3. We developed a technique to get better bit error rate in the case of high humidity and long transmitter-receiver distances when pulsed Terahertz signals are used:

- We exclude the frequency bands that cause most of the detection error due to atmospheric attenuation from the modulation process. As a result, we got better results when humidity is high and transmitterreceiver distance is long.

4. We studied the usage of continuous wave Terahertz wireless communications in omni-directional channels and developed rate adaptation algorithms to be used in Terahertz systems:

- The WiFi channel is used as the feedback channel while the Terahertz channel is used as the data channel. We developed two rate adaptation algorithms that use single or multiple channels for sending downlink data to users with different distances. The results show that fairness is maintained among the users and the users receive high data rates. For the single channel algorithm, each user receives 4 Gigabits/second and for the multiple channel algorithm data rates of 10 Gigabits/second are achieved.

5. We contributed to the NS3 network simulator: 
- We contributed the $\mathrm{C}++$ code for the directional and omni-directional channel models, tests suites, and an example to the NS3 community. The corresponding source codes can be found in Appendix E - Appendix H. 


\section{BIBLIOGRAPHY}

[1] http://www.batop.com/information/news/hpa.pdf.

[2] http://advancedphotonix.com/.

[3] http://ggweather.com/ccd/avgrh.htm.

[4] J Antes, S Koenig, D Lopez-Diaz, F Boes, A Tessmann, R Henneberger, O Ambacher, T Zwick, and I Kallfass. Transmission of an 8-PSK modulated 30 Gbit/s signal using an MMIC-based $240 \mathrm{GHz}$ wireless link. In Microwave Symposium Digest (IMS), 2013 IEEE MTT-S International, pages 1-3. IEEE, 2013.

[5] J Antes, S Konig, A Leuther, H Massler, J Leuthold, O Ambacher, and I Kallfass. $220 \mathrm{GHz}$ wireless data transmission experiments up to $30 \mathrm{Gbit} / \mathrm{s}$. In $\mathrm{Mi}$ crowave Symposium Digest (MTT), 2012 IEEE MTT-S International, pages 1-3. IEEE, 2012.

[6] J. Antes, J. Reichart, D. Lopez-Diaz, A Tessmann, F. Poprawa, F. Kurz, T. Schneider, H. Massler, and I. Kallfass. System concept and implementation of a mmW wireless link providing data rates up to $25 \mathrm{Gbit} / \mathrm{s}$. In 2011 IEEE International Conference on Microwaves, Communications, Antennas and Electronics Systems (COMCAS), pages 1-4, Nov 2011.

[7] Roger Appleby and H Bruce Wallace. Standoff detection of weapons and contraband in the $100 \mathrm{GHz}$ to $1 \mathrm{THz}$ region. IEEE transactions on antennas and propagation, 55(11):2944-2956, 2007. 
[8] Albrecht Bartels, Florian Hudert, Christof Janke, Thomas Dekorsy, and Klaus Köhler. Femtosecond time-resolved optical pump-probe spectroscopy at kilohertz-scan-rates over nanosecond-time-delays without mechanical delay line. Applied physics letters, 88:041117, 2006.

[9] Ivan Bradaric, Gerard T Capraro, and Donal D Weiner. Ultra wide band (UWB) interference-assessment and mitigation studies. Technical report, DTIC Document, 2006.

[10] S Cherry. Edholm's law of bandwidth. Spectrum, IEEE, 41(7):58-60, July 2004.

[11] D. Côté, JE Sipe, and H.M. van Driel. Simple method for calculating the propagation of terahertz radiation in experimental geometries. JOSA B, 20(6):1374-1385, 2003.

[12] G Ducournau, P Szriftgiser, D Bacquet, A Beck, T Akalin, E Peytavit, M Zaknoune, and JF Lampin. Optically power supplied Gbit/s wireless hotspot using $1.55 \mu \mathrm{m}$ THz photomixer and heterodyne detection at $200 \mathrm{GHz}$. Electronics letters, 46(19):1349-1351, 2010.

[13] Guillaume Ducournau, Pascal Szriftgiser, Alexandre Beck, Denis Bacquet, Fabio Pavanello, Emilien Peytavit, Mohammed Zaknoune, Tahsin Akalin, and J-F Lampin. Ultrawide-bandwidth single-channel $0.4-\mathrm{THz}$ wireless link combining broadband quasi-optic photomixer and coherent detection. 2014.

[14] M. Exter, C. Fattinger, and D. Grischkowsky. Terahertz time-domain spectroscopy of water vapor. Optic Letters, 14(20):1128-1130, 1989.

[15] Jerome Faist, Federico Capasso, Deborah L Sivco, Carlo Sirtori, Albert L Hutchinson, and Alfred Y Cho. Quantum cascade laser. Science, 264(5158):553-556, 1994. 
[16] John Federici and Lothar Moeller. Review of terahertz and subterahertz wireless communications. Journal of Applied Physics, 107(11), 2010.

[17] John F Federici, Dale Gary, Robert Barat, and David Zimdars. THz standoff detection and imaging of explosives and weapons. In Defense and Security, pages 75-84. International Society for Optics and Photonics, 2005.

[18] Michael Feiginov, Cezary Sydlo, Oleg Cojocari, and Peter Meissner. Resonanttunnelling-diode oscillators operating at frequencies above $1.1 \mathrm{THz}$. Applied Physics Letters, 99(23), 2011.

[19] MJ Fice, E Rouvalis, F van Dijk, A Accard, F Lelarge, CC Renaud, G Carpintero, and AJ Seeds. 146-GHz millimeter-wave radio-over-fiber photonic wireless transmission system. Optics express, 20(2):1769-1774, 2012.

[20] T Furuya, K Horita, Christopher T Que, K Yamamoto, F Miyamaru, S Nishizawa, and M Tani. Development of fast scan THz-TDS system by using repetition rate tunable femtosecond laser. In Infrared Millimeter and Terahertz Waves (IRMMW-THz), 2010 35th International Conference on, pages 1-2. IEEE, 2010.

[21] Chong Han and Ian F Akyildiz. Distance-aware multi-carrier (DAMC) modulation in terahertz band communication. In 2014 IEEE International Conference on Communications (ICC), pages 5461-5467. IEEE, 2014.

[22] A. Hirata, T. Kosugi, H. Takahashi, J. Takeuchi, K. Murata, N Kukutsu, Y. Kado, S. Okabe, T. Ikeda, F. Suginosita, et al. 5.8-km 10-Gbps data transmission over a 120-GHz-band wireless link. In 2010 IEEE International Conference on Wireless Information Technology and Systems (ICWITS),, pages 1-4. IEEE, 2010.

[23] Akihiko Hirata, Toshihiko Kosugi, Nicholas Meisl, Tsugumichi Shibata, and 
Tadao Nagatsuma. High-directivity photonic emitter using photodiode module integrated with HEMT amplifier for 10-Gbit/s wireless link. IEEE Transactions on Microwave Theory and Techniques, 52(8):1843-1850, 2004.

[24] Akihiko Hirata, Toshihiko Kosugi, Hiroyuki Takahashi, Ryouichi Yamaguchi, Fumito Nakajima, Tomofumi Furuta, Hioshi Ito, Hirohiko Sugahara, Yasuhiro Sato, and Tadao Nagatsuma. 120-GHz-band millimeter-wave photonic wireless link for $10-\mathrm{Gb} / \mathrm{s}$ data transmission. IEEE Transactions on Microwave Theory and Techniques, 54(5):1937-1944, 2006.

[25] Daquan Huang, Tim R LaRocca, M-CF Chang, Lorene Samoska, Andy Fung, Richard L Campbell, and Michael Andrews. Terahertz CMOS frequency generator using linear superposition technique. IEEE Journal of Solid-State Circuits, 43(12):2730-2738, 2008.

[26] K Ishigaki, M Shiraishi, S Suzuki, M Asada, N Nishiyama, and S Arai. Direct intensity modulation and wireless data transmission characteristics of terahertz-oscillating resonant tunnelling diodes. Electronics letters, 48(10):582-583, 2012.

[27] C. Jastrow, K. Munter, R. Piesiewicz, T Kurner, M. Koch, and T. KleineOstmann. $300 \mathrm{GHz}$ transmission system. Electronics Letters, 44(3):213-214, January 2008.

[28] C Jastrow, S Priebe, B Spitschan, J Hartmann, M Jacob, T Kürner, T Schrader, and T Kleine-Ostmann. Wireless digital data transmission at 300 GHz. Electronics letters, 46(9):661-663, 2010.

[29] J Jornet and I Akyildiz. Femtosecond-long pulse-based modulation for terahertz band communication in nanonetworks. 2014.

[30] Ingmar Kallfass, Jochen Antes, Thomas Schneider, Fabian Kurz, Daniel 
Lopez-Diaz, Sebastian Diebold, Hermann Massler, Arnulf Leuther, and Axel Tessmann. All active MMIC-based wireless communication at $220 \mathrm{GHz}$. IEEE Transactions on Terahertz Science and Technology, 1(2):477-487, November 2011.

[31] Youngchan Kim and Dae-Su Yee. High-speed terahertz time-domain spectroscopy based on electronically controlled optical sampling. Optics letters, $35(22): 3715-3717,2010$.

[32] G Kh Kitaeva. Terahertz generation by means of optical lasers. Laser Physics Letters, 5(8), 2008.

[33] Thomas Kleine-Ostmann and Tadao Nagatsuma. A review on terahertz communications research. Journal of Infrared, Millimeter, and Terahertz Waves, $32(2): 143-171,2011$.

[34] S. Koenig, D. Lopez-Diaz, J. Antes, F. Boes, R. Henneberger, A. Leuther, A. Tessmann, R. Schmogrow, D. Hillerkuss, R. Palmer, T. Zwick, C. Koos, W. Freude, O. Ambacher, J. Leuthold, and I. Kallfass. Wireless sub-THz communication system with high data rate. Nature Photonics, 7:977-981, October 2013.

[35] Rüdeger Köhler, Alessandro Tredicucci, Fabio Beltram, Harvey E Beere, Edmund H Linfield, Giles Davies, David A Ritchie, Rita C Iotti, and Fausto Rossi. Terahertz semiconductor-heterostructure laser. Nature, 417(6885):156$159,2002$.

[36] Sushil Kumar and A.W.M. Lee. Resonant-phonon terahertz quantum-cascade lasers and video-rate terahertz imaging. Selected Topics in Quantum Electronics, IEEE Journal of, 14(2):333-344, March 2008.

[37] Thomas Kürner. Towards future thz communications systems. Terahertz 
Science and Technology, 5(1), 2012.

[38] Bin Lu, Wei Huang, C Lin, and C Wang. A 16QAM modulation based 3Gbps wireless communication demonstration system at $0.34 \mathrm{THz}$ band. In Infrared, Millimeter, and Terahertz Waves (IRMMW-THz), 2013 38th International Conference on, pages 1-2. IEEE, 2013.

[39] L. Moeller, John Federici, and Ke Su. THz wireless communications: $2.5 \mathrm{~Gb} / \mathrm{s}$ error-free transmission at $625 \mathrm{GHz}$ using a narrow-bandwidth $1 \mathrm{~mW} \mathrm{THz}$ source. In 2011 XXXth URSI General Assembly and Scientific Symposium, pages $1-4,2011$.

[40] L Moeller, A Shen, C Caillaud, and M Achouche. Enhanced THz generation for wireless communications using short optical pulses. In Infrared, Millimeter, and Terahertz Waves (IRMMW-THz), 2013 38th International Conference on, pages 1-3. IEEE, 2013.

[41] Tatsuya Mori, Hikaru Igawa, and Seiji Kojima. Progress of ultrafast terahertz time-domain spectroscopy: Raman inactive soft mode in quantum paraelectric SrTiO3. In IOP Conference Series: Materials Science and Engineering, volume 54, page 012006. IOP Publishing, 2014.

[42] P Mukherjee and B Gupta. Terahertz (THz) frequency sources and antennasa brief review. International Journal of Infrared and Millimeter Waves, 29(12):1091-1102, 2008.

[43] Tadao Nagatsuma, Shogo Horiguchi, Yusuke Minamikata, Yasuyaki Yoshimizu, Shinataro Hisatake, Shigeru Kuwano, Naoto Yoshimoto, Jun Terada, and Hiroyuki Takahashi. Terahertz wireless communications based on photonics technologies. Optics express, 21(20):23736-23747, 2013.

[44] Tadao Nagatsuma, Akira Kaino, Shinataro Hisatake, Katsuhiro Ajito, Ho-Jin 
Song, Atsushi Wakatsuki, Yoshifumi Muramoto, Naoya Kukutsu, and Yuichi Kado. Continuous-wave terahertz spectroscopy system based on photodiodes. PIERS Online, 6(4):390-394, 2010.

[45] Dominic C O’Brien, G Faulkner, Kalok Jim, Emmanuel B Zyambo, David J Edwards, Mark Whitehead, Paul Stavrinou, Gareth Parry, Jacques Bellon, Martin J Sibley, et al. High-speed integrated transceivers for optical wireless. IEEE Communications Magazine, 41(3):58-62, 2003.

[46] Kaveh Pahlavan and Allen H Levesque. Wireless information networks, volume 93. John Wiley \& Sons, 2005.

[47] Xiaodan Pang, Antonio Caballero, Anton Dogadaev, Valeria Arlunno, Robert Borkowski, Jesper S Pedersen, Lei Deng, Fotini Karinou, Fabien Roubeau, Darko Zibar, et al. 100 Gbit/s hybrid optical fiber-wireless link in the w-band (75-110 GHz). Optics express, 19(25):24944-24949, 2011.

[48] Radoslaw Piesiewicz, Christian Jansen, Daniel Mittleman, Thomas KleineOstmann, Martin Koch, and Thomas Kurner. Scattering analysis for the modeling of thz communication systems. IEEE Transactions on Antennas and Propagation, 55(11):3002-3009, 2007.

[49] J. K. Rhee, Hisashi Kobayashi, and Warren S. Warren. Optical frequency domain differential phase shift keying in femtosecond-pulse spectral modulation systems. Journal of Lightwave Technology, 15(12):2214-2222, 1997.

[50] June-Koo Rhee, Hisashi Kobayashi, and Warren S. Warren. Frequencydomain differential phase shift keying in femtosecond spread-time WDM/TDM systems. In Conf. Inform. Sci. Syst, pages 1107-1112, 1996.

[51] A Rogalski and F Sizov. Terahertz detectors and focal plane arrays. Optoelectronics review, 19(3):346-404, 2011. 
[52] Laurence S Rothman, John Schroeder, and Kuilian Tang. Javahawks (java hitran atmospheric workstation) manual. 2004.

[53] LS Rothman, IE Gordon, Y Babikov, A Barbe, D Chris Benner, PF Bernath, Manfred Birk, L Bizzocchi, V Boudon, LR Brown, et al. The hitran2012 molecular spectroscopic database. Journal of Quantitative Spectroscopy and Radiative Transfer, 130:4-50, 2013.

[54] Daryoosh Saeedkia. Handbook of terahertz technology for imaging, sensing and communications. Elsevier, 2013.

[55] S Sarkozy, M Vukovic, JG Padilla, J Chang, G Tseng, P Tran, P Yocom, W Yamasaki, KMKH Leong, and W Lee. Demonstration of a G-band transceiver for future space crosslinks. 2013.

[56] B Sartorius, H Roehle, H Künzel, J Böttcher, M Schlak, D Stanze, H Venghaus, and M Schell. All-fiber terahertz time-domain spectrometer operating at $1.5 \mu \mathrm{m}$ telecom wavelengths. Optics express, 16(13):9565-9570, 2008.

[57] Hani Sherry, Janus Grzyb, Yan Zhao, Richard Al Hadi, Andreia Cathelin, Andreas Kaiser, and Ullrich Pfeiffer. A 1kpixel CMOS camera chip for 25fps real-time terahertz imaging applications. In Solid-State Circuits Conference Digest of Technical Papers (ISSCC), 2012 IEEE International, pages 252-254. IEEE, 2012.

[58] Peter H Siegel. Terahertz technology. IEEE Transactions on microwave theory and techniques, 50(3):910-928, 2002.

[59] Cambridge Philosophical Society. Transactions of the Cambridge Philosophical Society, volume 5. University Press, 1835.

[60] H.J. Song, K. Ajito, A. Hirata, A. Wakatsuki, T. Furuta, N. Kukutsu, and T. Nagatsuma. Multi-gigabit wireless data transmission at over 200-GHz. In 
34th International Conference on Infrared, Millimeter, and Terahertz Waves, 2009. IRMMW-THz 2009, pages 1-2, 2009.

[61] H.J. Song, K. Ajito, Y. Muramoto, and A. Wakatasuki. 24 Gbit/s data transmission in $300 \mathrm{GHz}$ band for future terahertz communications. Electronics Letters, 48(15):953-954, July 2012.

[62] Ho-Jin Song, Jae-Young Kim, Katsuhiro Ajito, Makoto Yaita, and Naoya Kukutsu. Fully integrated ASK receiver MMIC for terahertz communications at $300 \mathrm{GHz}$. IEEE Transactions on Terahertz Science and Technology, $3(4): 445-452,2013$.

[63] Ho-Jin Song and Tadao Nagatsuma. Present and future of terahertz communications. IEEE Transactions on Terahertz Science and Technology, 1(1):256$263,2011$.

[64] DF Swinehart. The beer-lambert law. Journal of chemical education, $39(7): 333,1962$.

[65] S. Verghese, K. A. McIntosh, S. Calawa, W. F. DiNatale, E. K. Duerr, and K. A. Molvar. Generation and detection of coherent terahertz waves using two photomixers. Applied Physics Letters, 73(26):3824-3826, Dec 1998.

[66] John H Van Vleck and Victor F Weisskopf. On the shape of collisionbroadened lines. Reviews of Modern Physics, 17(2-3):227, 1945.

[67] Cheng Wang, Changxing Lin, Qi Chen, Bin Lu, Xianjin Deng, and Jian Zhang. A 10-Gbit/s wireless communication link using 16-QAM modulation in 140-GHz band. IEEE Transactions on Microwave Theory and Techniques, 61(7):2737-2746, July 2013.

[68] Benjamin S Williams. Terahertz quantum-cascade lasers. Nature photonics, 1(9):517-525, 2007. 
[69] Ruth M Woodward, Bryan E Cole, Vincent P Wallace, Richard J Pye, Donald D Arnone, Edmund H Linfield, and Michael Pepper. Terahertz pulse imaging in reflection geometry of human skin cancer and skin tissue. Physics in medicine and biology, 47(21):3853, 2002.

[70] Dwight Woolard, Peiji Zhao, Christopher Rutherglen, Zhen Yu, Peter Burke, Steven Brueck, and Andreas Stintz. Nanoscale imaging technology for THzfrequency transmission microscopy. International Journal of High Speed Electronics and Systems, 18(01):205-222, 2008.

[71] Xianbin Yu, Ying Chen, Michael Galili, Toshio Morioka, Peter Uhd Jepsen, and Leif K Oxenlowe. The prospects of ultra-broadband THz wireless communications. In Transparent Optical Networks (ICTON), 2014 16th International Conference on, pages 1-4. IEEE, 2014.

[72] J Axel Zeitler and Lynn F Gladden. In-vitro tomography and non-destructive imaging at depth of pharmaceutical solid dosage forms. European Journal of Pharmaceutics and Biopharmaceutics, 71(1):2-22, 2009.

[73] Bo Zhang, Yong-Zhong Xiong, Lei Wang, and Sanming Hu. A switch-based ASK modulator for $10 \mathrm{Gbps} 135 \mathrm{GHz}$ communication by 0.13 MOSFET. IEEE Microwave and Wireless Components Letters, 22(8):415-417, 2012.

[74] Xi-Cheng Zhang and Jingzhou Xu. Introduction to THz wave photonics. Springer, 2010. 
Appendix A

TIME DOMAIN SIGNALS

\author{
$\%$ \\ \% Filtering the time domain data to delete the \\ $\%$ reflection effects \\ $\%$ \\ function $x_{-}$filtered $=$filter_time $(x)$ \\ $\% \mathrm{x}$ is the time domain signal \\ index $=$ find $(\operatorname{abs}(x)>0.1 *(\max (\operatorname{abs}(x))))$; \\ index_begin = index $(1)-100$; \\ index_end $=$ index_begin +400 ; \\ palse_len $=$ index_end - index_begin ; \\ $x_{-}$filtered $=\operatorname{zeros}(1$, length $(x))$;

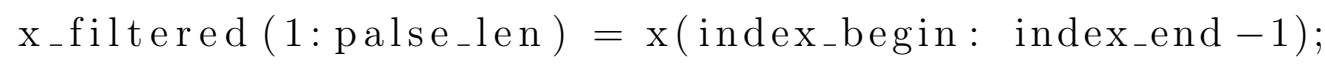 \\ $\mathrm{x}_{-}$filtered $($palse_len +1 : end $)=0$;
}

end 
Appendix B

ATMOSPHERIC ATTENUATION COEFFICIENT

$\%$ find the absorption coeffiecient based on
$\%$ Hitran database and WW line shape model
$\%$

function [total_absorption] = absorption(Freq, rh)

$\%$ Freq is the frequency elements

\%rh is the relative humidity of the air

$\% c$ is the speed of light

$\mathrm{c}=2.997925 \mathrm{e} 8 ; \quad \%$ speed of light $(\mathrm{m} / \mathrm{s})$

\%wavenumber of each correspond frequency

$\mathrm{v}=($ Freq $\cdot / \mathrm{c}) \cdot * 0.01$

$\% \mathrm{~T}$ in kelvin, pd and pw in pa

$\mathrm{p}=101325 ; \% \mathrm{pa}$

$\%$ millibar saturation pressure for $\mathrm{T}=21 \mathrm{C}$

$\mathrm{p}_{-} \mathrm{sat}=24.8680 ;$

$\mathrm{pv}=\mathrm{rh} / 100 * \mathrm{p}_{\text {- sat }} ; \% \mathrm{millibar}$

$\mathrm{pv}=\mathrm{pv} * 100 ; \%$ pressure of water vapor in pa

$\mathrm{pd}=\mathrm{p}-\mathrm{pv} ; \%$ pressure of dry air in pa 
$\mathrm{T}=296 ; \%$ temperature

\%Water absorption

$$
\begin{aligned}
& \text { if }(\mathrm{rh} \sim 0) \\
& \quad \text { data }=\text { importdata(' absorption_table/water_hitran.txt') } ;
\end{aligned}
$$

q_water_vapor $=(0.622 * \mathrm{pv}) /(\mathrm{pd})$;

v0_water $=\operatorname{data}(:, 1)$.' ;

s_water $=\operatorname{data}(:, 2)$.'

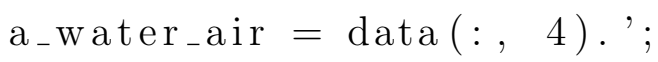

$a_{-}$water_self $=\operatorname{data}(:, 5) . '$;

a_water $=\left(1-\right.$ q_water_vapor $_{-} *$ a_water_air $_{-}+\ldots$

q-water_vapor .* a_water_self;

$$
\begin{aligned}
& \text { for } \quad v_{\text {_ind }}=1 \text { : length }(\mathrm{v}) \\
& \mathrm{k}_{-} \text {water }\left(\mathrm{v}_{-} \text {ind }\right)=0 \text {; } \\
& \text { ind }=1 \text {; } \\
& \text { for ind }=1 \text { : length (v0_water }) \\
& \text { orW line shape } \\
& \mathrm{k}_{\text {_water }}\left(\mathrm{v}_{\text {_ind }}\right)=\mathrm{k}_{\text {- water }}\left(\mathrm{v}_{-} \text {ind }\right)+\ldots \\
& \text { (s_water (ind)/pi )... } \\
& *\left(\mathrm{v}\left(\mathrm{v} \_ \text {ind }\right) / \mathrm{v} 0 \text { _water }(\text { ind })\right) \ldots \\
& \text { ^ } 2 *(\text { a }- \text { water (ind }) / \ldots \\
& \left(\left(\mathrm{v}\left(\mathrm{v} \_ \text {ind }\right)-\mathrm{v} 0 \text { _water }(\text { ind })\right) \ldots\right.
\end{aligned}
$$




$$
\begin{aligned}
& \left.\left.{ }^{\wedge} 2+\left(\text { a_water }_{-} \text {ind }\right)\right)^{\wedge} 2\right) \ldots \\
& +\mathrm{a}_{-} \text {water }(\text { ind }) / \ldots \\
& \left(\left(\mathrm{v}\left(\mathrm{v}_{-} \text {ind }\right)+\mathrm{v} 0 \text {-water }(\text { ind })\right)^{\wedge} 2+\ldots\right. \\
& \text { a_water } \left.\left.(\text { ind }) \wedge^{\wedge} 2\right)\right)
\end{aligned}
$$

end

end

$$
\begin{aligned}
& \mathrm{Mv}=0.018016 ; \\
& \mathrm{R}=8.314 ; \\
& \text { avogadro }=6.02214129 \mathrm{e}+23 ; \\
& \% \text { unit } \mathrm{kg} / \mathrm{m} 3 \longrightarrow 1 \mathrm{gr} \text { water }=0.0555084350618 \text { moles } \\
& \text { vapor_density }=(\mathrm{pv} * \mathrm{Mv}) /(\mathrm{R} * \mathrm{~T}) ; \\
& \text { vapor_density }=\text { vapor_density } * 0.001 * \ldots \\
& \quad 0.0555084350618 * \text { avogadro } \% \text { molecules } / \mathrm{cm} 3
\end{aligned}
$$

water_absorption_coeff $=$ vapor_density $*$ k $_{-}$water $;$

end

\%Oxygen absorption -

data_oxygen $=\ldots$

importdata ('absorption_table/oxygen_hitran .txt');

v0_oxygen $=$ data_oxygen $(:, 1) . '$;

s_oxygen $=$ data_oxygen $(:, 2) . '$;

a_oxygen_air = data_oxygen $(:, 4)$. ';

a_oxygen_self $=$ data_oxygen $(:, 5)$.' ;

q_oxygen $=0.21$; 


$$
\begin{aligned}
& \text { a_oxygen }=\left(1-\text { q_oxygen }_{-} . * \ldots\right. \\
& \text { a_oxygen_air + q_oxygen.* a_oxygen_self; } \\
& \text { for } \quad \mathrm{v}_{-} \text {ind }=1 \text { : } \operatorname{length}(\mathrm{v}) \\
& \text { k_oxygen }\left(\mathrm{v}_{\text {_ind }}\right)=0 \text {; } \\
& \text { for ind }=1 \text { : length (v0_oxygen }) \\
& \mathrm{k}_{-} \text {oxygen }\left(\mathrm{v}_{-} \text {ind }\right)=\mathrm{k}_{-} \text {oxygen }\left(\mathrm{v}_{-} \text {ind }\right)+\ldots \\
& \text { (s_oxygen (ind)/pi )... } \\
& *\left(\mathrm{v}\left(\mathrm{v}_{\text {_ind }}\right) / \mathrm{v} 0 \_ \text {oxygen }(\text { ind })\right)^{\wedge} 2 \ldots \\
& *(\text { a_oxygen }(\text { ind }) / \ldots \\
& \left(\left(\mathrm{v}\left(\mathrm{v}_{-} \text {ind }\right)-\mathrm{v} 0 \text { _oxygen }(\text { ind })\right)^{\wedge} 2 \ldots\right. \\
& \left.+ \text { a_oxygen }(\text { ind })^{\wedge} 2\right) \quad+\ldots \\
& \text { a_oxygen (ind ) / ( v ( v_ind ) ... } \\
& \left.+\mathrm{v} 0 \_ \text {oxygen }(\text { ind })\right)^{\wedge} 2 \ldots \\
& \text { +a_oxygen (ind )^2)); }
\end{aligned}
$$

end

end

oxygen_density $=4.923 \mathrm{e} 19 ; \%$ molecules $/ \mathrm{cm} 3$

oxygen_absorption $=$ oxygen_density $* \mathrm{k}_{-}$oxygen ;

$\%$ N2 absorption

data_N2= importdata ('absorption_table/N2_hitran.txt') ;

v0_N2 = data_N2 $(:, 1)$.' $^{\prime}$

$\mathrm{s}_{-} \mathrm{N} 2=$ data_N2 $(:, 2)$. ' $^{\prime}$

$\mathrm{a}_{-} \mathrm{N} 2$-air $=$ data_N2 $(:, 4)$.' $^{\prime}$ 


$$
\begin{aligned}
& \mathrm{a}_{-} \mathrm{N} 2{ }_{-} \text {self }=\text { data_N2 }(:, 5) \text {.' ; } \\
& \text { q_N2 }=78.084 * 0.01 \text {; } \\
& \mathrm{a}_{-} \mathrm{N} 2=\left(1-\mathrm{q}_{-} \mathrm{N} 2\right) \cdot * \mathrm{a}_{-} \mathrm{N} 22_{-} \mathrm{air}+\mathrm{q}_{-} \mathrm{N} 2 \cdot * \mathrm{a}_{-} \mathrm{N} 22_{-} \text {self } ; \\
& \text { for } \quad \mathrm{v}_{-} \text {ind }=1 \text { : } \operatorname{length}(\mathrm{v}) \\
& \mathrm{k}_{-} \mathrm{N} 2\left(\mathrm{v}_{-} \text {ind }\right)=0 \text {; } \\
& \text { for ind }=1: \text { length }\left(\mathrm{v} 0 \_\mathrm{N} 2\right) \\
& \mathrm{k}_{-} \mathrm{N} 2\left(\mathrm{v}_{-} \text {ind }\right)=\mathrm{k}_{-} \mathrm{N} 2\left(\mathrm{v}_{-} \text {ind }\right)+\ldots \\
& \left(\mathrm{s}_{-} \mathrm{N} 2(\text { ind }) / \mathrm{pi}\right) * \ldots \\
& \left(\mathrm{v}\left(\mathrm{v}_{-} \text {ind }\right) / \mathrm{v} 0 \_\mathrm{N} 2(\text { ind })\right)^{\wedge} 2 \ldots \\
& \text { * }\left(\mathrm{a}_{-} \mathrm{N} 2(\text { ind }) /\left(\left(\mathrm{v}\left(\mathrm{v} \_ \text {ind }\right) \ldots\right.\right.\right. \\
& \left.\left.-\mathrm{v} 0 \_\mathrm{N} 2(\text { ind })\right)^{\wedge} 2+\mathrm{a}_{-} \mathrm{N} 2(\text { ind }) \wedge 2\right) \ldots \\
& + \text { a_N2(ind }) / \ldots \\
& \left(\left(\mathrm{v}\left(\mathrm{v}_{-} \text {ind }\right)+\mathrm{v} 0 \_\mathrm{N} 2(\text { ind })\right) \wedge \ldots\right. \\
& \left.\left.2+\mathrm{a}_{-} \mathrm{N} 2(\text { ind })^{\wedge} 2\right)\right) \text {; }
\end{aligned}
$$

end

end

$\%$ N2_density $=0.0638 * 0.001 *$ avogadro $\%$ molecules $/ \mathrm{cm} 3$

$\mathrm{N} 2$ _density $=4.9229 \mathrm{e} 19 ;$

$\mathrm{N} 2$ _absorption $=\mathrm{N} 2$ _density $* \mathrm{k}_{-} \mathrm{N} 2 ; \%$ molecules $/ \mathrm{cm} 3$

$\% \operatorname{co} 2$ absorption

data_co $2=$ importdata(' absorption_table/co $2_{-}$hitran.txt') ;

$\mathrm{v}_{0} \mathrm{co} 2=\mathrm{data}_{-} \operatorname{co} 2(:, \quad 1) \cdot{ }^{\prime} ;$

$\mathrm{s}_{-} \mathrm{co} 2=$ data_co $2(:, \quad 2) \cdot{ }^{\prime}$; 


$$
\begin{aligned}
& a_{-} \operatorname{co} 2_{-} \text {air }=\text { data_co } 2(:, 4) . ' ; \\
& a_{-} \operatorname{co} 2_{-} \text {self }=\text { data_co } 2(:, \quad 5) . ' ;
\end{aligned}
$$

$\mathrm{q}_{-} \operatorname{co} 2=0.035 * 0.01$

$\mathrm{a}_{-} \operatorname{co} 2=\left(1-\mathrm{q}_{-} \operatorname{co} 2\right) \cdot * \mathrm{a}_{-} \operatorname{co} 2{ }_{-} \mathrm{air}+\mathrm{q}_{-} \operatorname{co} 2 * \mathrm{a}_{-} \mathrm{co} 2{ }_{-} \mathrm{self} ;$

$$
\begin{aligned}
& \text { for } \quad \mathrm{v}_{-} \text {ind }=1 \text { : } \operatorname{length}(\mathrm{v}) \\
& \mathrm{k}_{-} \operatorname{co} 2\left(\mathrm{v}_{-} \mathrm{ind}\right)=0 \text {; } \\
& \text { for ind }=1: \operatorname{length}\left(\mathrm{v} 0 \_\mathrm{co} 2\right) \\
& \mathrm{k}_{-} \mathrm{co} 2\left(\mathrm{v}_{-} \mathrm{ind}\right)=\mathrm{k}_{-} \operatorname{co} 2\left(\mathrm{v}_{-} \text {ind }\right)+\left(\mathrm{s}_{-} \operatorname{co} 2(\text { ind }) / \mathrm{pi}\right) * \ldots \\
& \left(\mathrm{v}\left(\mathrm{v}_{-} \text {ind }\right) / \mathrm{v}_{0}{ }_{-} \mathrm{co} 2(\text { ind })\right)^{\wedge} 2 *\left(\mathrm{a}_{-} \mathrm{co} 2(\text { ind }) / \ldots\right. \\
& \left(\left(\mathrm{v}\left(\mathrm{v}_{-} \text {ind }\right)-\mathrm{v}_{0} 0_{-} \mathrm{co} 2(\text { ind })\right)^{\wedge} 2 \ldots\right. \\
& \left.+a_{-} \operatorname{co} 2(\text { ind })^{\wedge} 2\right)+a_{-} \operatorname{co} 2(\text { ind }) \ldots \\
& /\left(\left(v_{(}\left(v_{-} \text {ind }\right)+v_{0}{ }_{-} \operatorname{co} 2(\text { ind })\right)^{\wedge} 2+\ldots\right. \\
& \left.\left.\mathrm{a}_{-} \operatorname{co} 2(\text { ind })^{\wedge} 2\right)\right) \text {; }
\end{aligned}
$$

end

end

co2_density $=2.4617$ e19; \%molecules $/ \mathrm{cm} 3$

co2_absorption $=$ co $2{ }_{-}$density $* \mathrm{k}_{-} \mathrm{co} 2$;

\%TOTAL ABSORPTION

freq_ind $=$ find $($ Freq $<=2 \mathrm{e} 12,1$, 'last,$\quad)$;

if $\left(\mathrm{rh}^{\sim}=0\right)$

$$
\text { total_absorption }=\text { oxygen_absorption }+\ldots
$$

water_absorption_coeff $+\ldots$

N2_absorption + co2_absorption; 


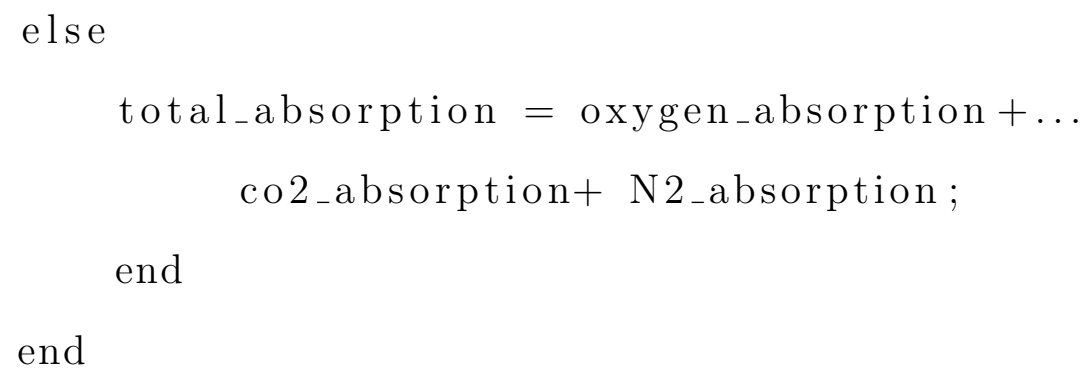


Appendix C

RAISED COSINE FILTER

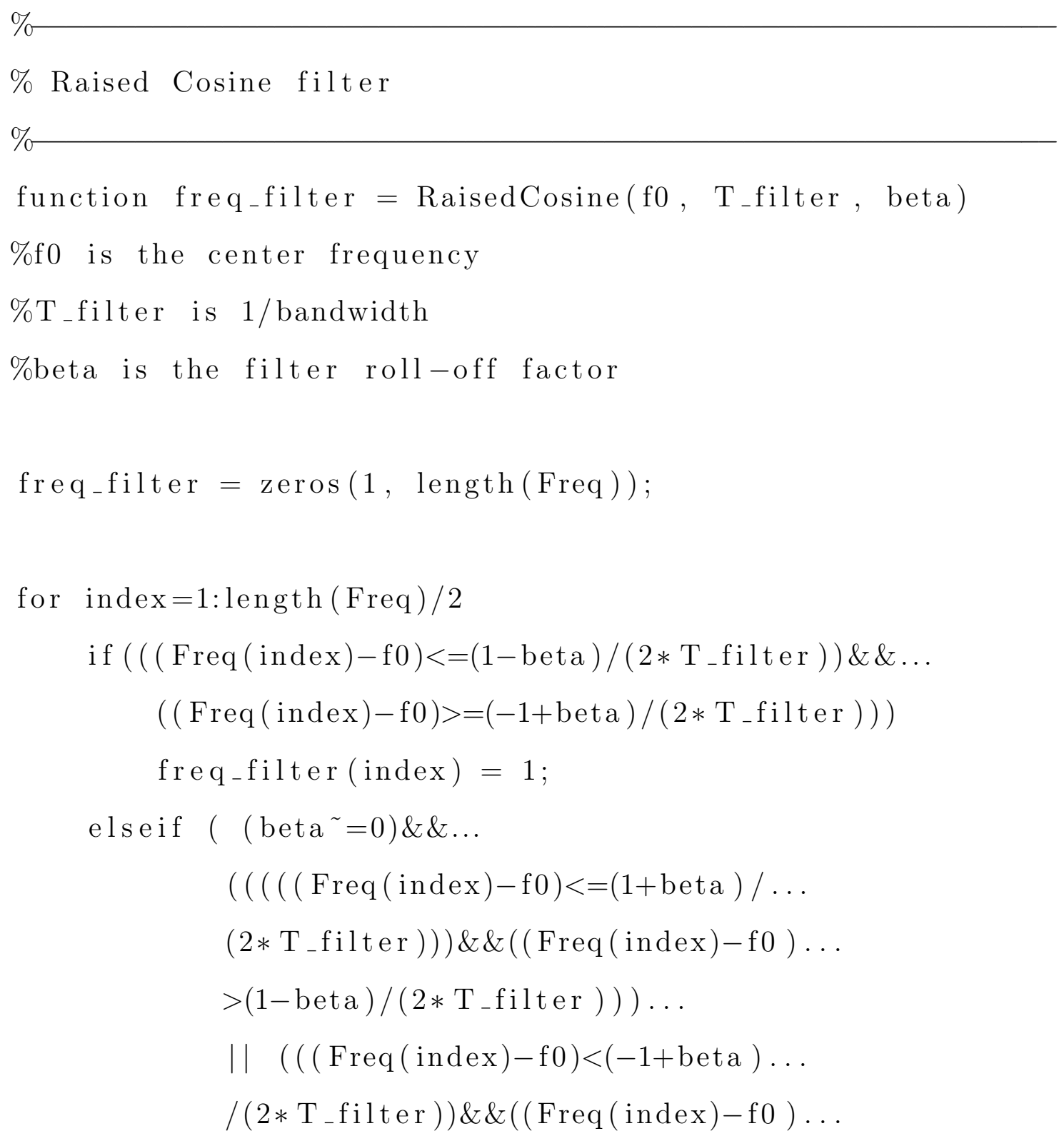




$$
\begin{aligned}
& \left.\left.\left.\left.>=(-1-\text { beta }) /\left(2 * T_{-} \text {filter }\right)\right)\right)\right)\right) \\
& \text { freq-filter }(\text { index })=\ldots \\
& 1 / 2 *\left(1+\cos \left(\left(\mathrm{pi} * \mathrm{~T}_{-} \text {filter } / \text { beta }\right) \ldots\right.\right. \\
& \text { *(abs (Freq }(\text { index })-\mathrm{f} 0)-\ldots \\
& \left.\left.\left.\left((1-\text { beta }) /\left(2 * T_{\text {_filter }}\right)\right)\right)\right)\right) \text {; } \\
& \text { freq-filter }(\text { index })=0 \text {; }
\end{aligned}
$$

else

end

end

end 
Appendix D

FD-DPSK MODULATION

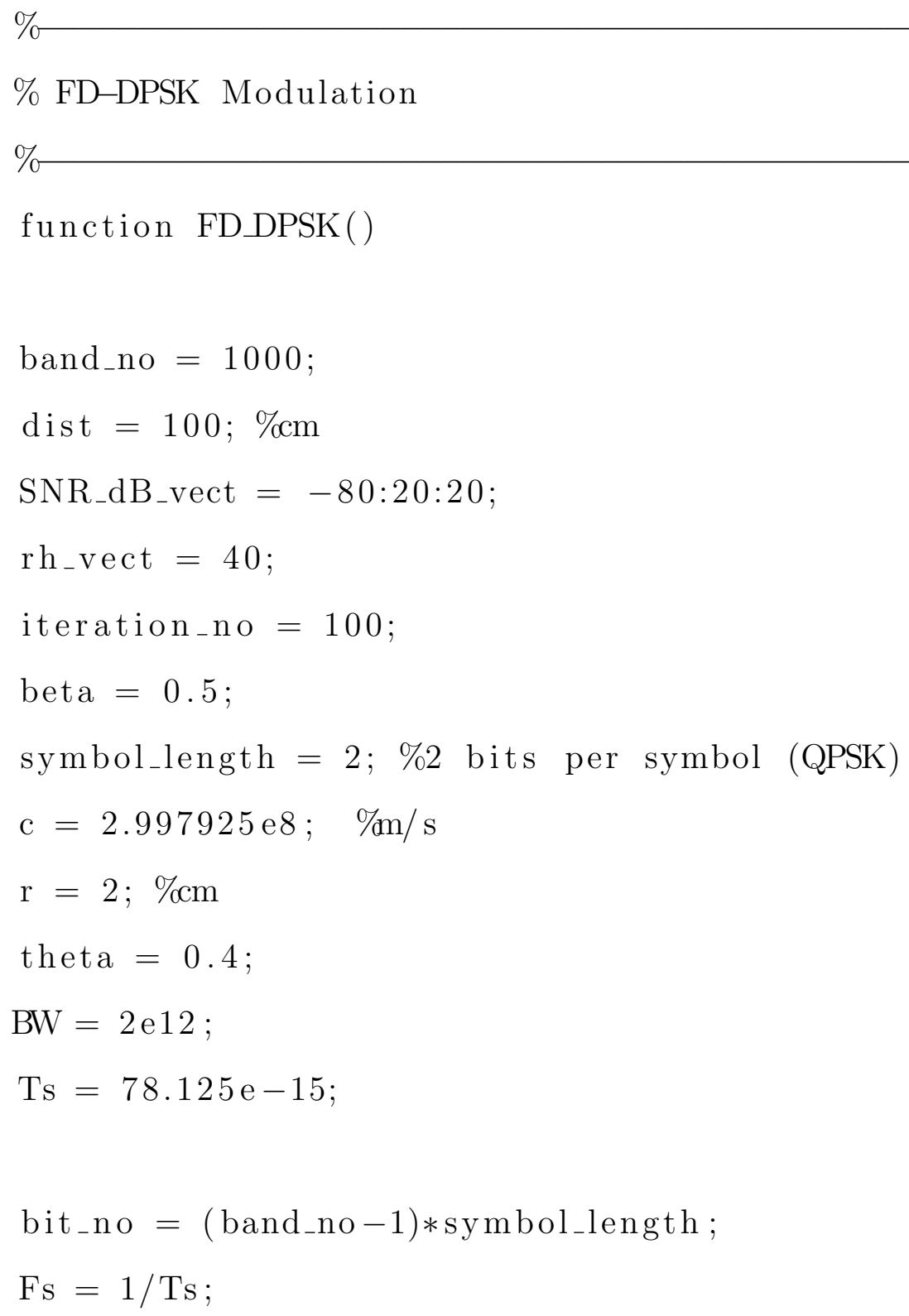




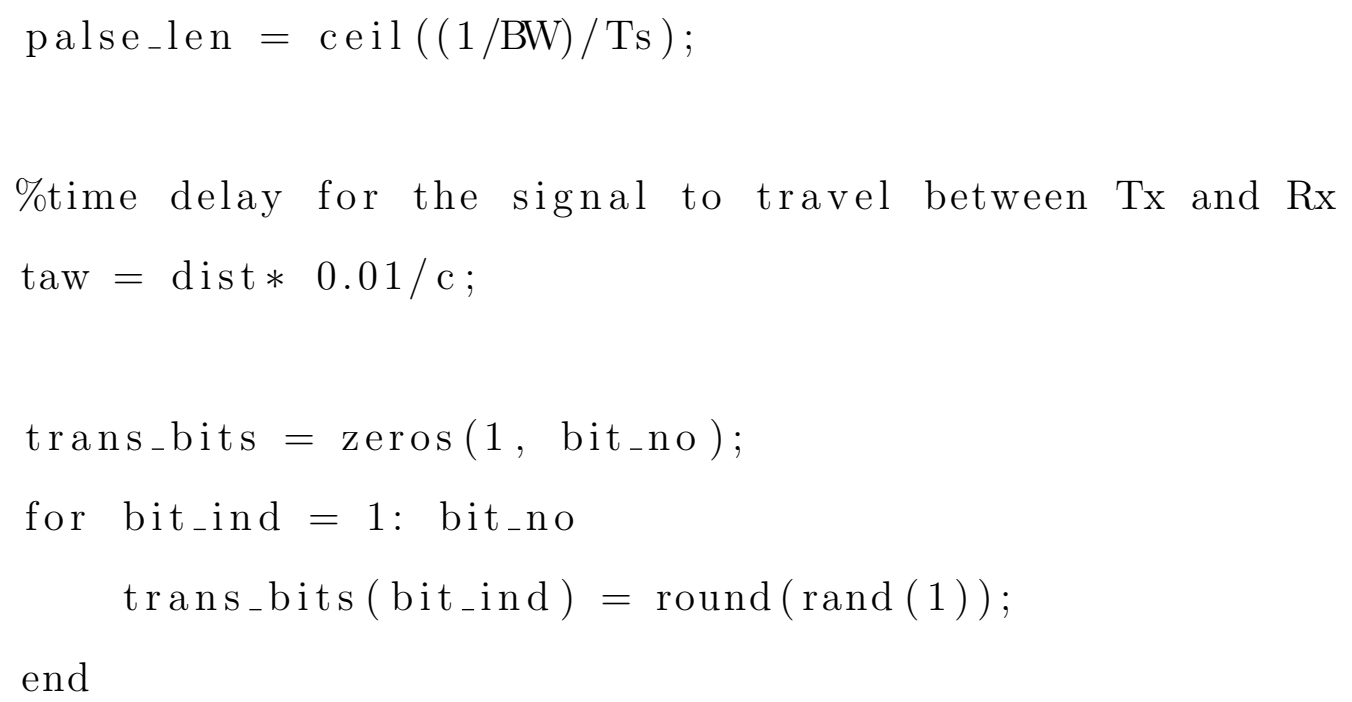


\%creat the freq-filter to use at the receiver

$\mathrm{T}_{-}$filter $=1 /$ filter_size;

freq_filter $=$ zeros(band_no, N);

for index $=1$ : band_no

FirstInd $=($ index -1$) *$ filter_step +1 ;

LastInd $=$ FirstInd+filter_step -1 ;

MidIndex $=$ round $(($ LastInd - FirstInd $) / 2)+$ FirstInd ;

freq-filter (index, : $)=\ldots$

RaisedCosine (Freq(MidIndex), T_filter, Freq, beta);

freq-filter (index, end:-1:end-round $(\mathrm{N} / 2))=\ldots$

freq_filter (index, $1: \operatorname{round}(\mathrm{N} / 2)+1)$;

end

BER_vect $=\operatorname{zeros}\left(\right.$ length $($ rh_vect $)$, length $\left.(\text { SNR_dB_vect })_{-}\right)$;

for $\mathrm{rh}_{-}$ind $=1$ : $\operatorname{length}\left(\mathrm{rh}_{-}\right.$vect $)$

$\mathrm{rh}=\mathrm{rh}_{-}$vect $\left(\mathrm{rh}_{-}\right.$ind $)$;

$\operatorname{disp}\left(\left[' R H=', \operatorname{num} 2 \operatorname{str}\left(\operatorname{rh}_{-} \operatorname{vect}\left(\mathrm{rh}_{-}\right.\right.\right.\right.$ind $\left.\left.\left.)\right)\right]\right)$;

[total_absorption $]=$ absorption (Freq, rh, c);

$\operatorname{coef}=\operatorname{zeros}(1, \mathrm{~N})$;

$\mathrm{H}=\mathrm{zeros}(1, \mathrm{~N})$;

for Freq_index $=1: \mathrm{N}$

$\operatorname{coef}($ Freq_index $)=(r /(r+d i s t * \operatorname{tand}(\text { theta })))^{\wedge} 2 \ldots$

* $\exp (-1 *($ total_absorption (Freq_index $) *$ dist $))$;

$\mathrm{H}($ Freq_index $)=\operatorname{coef}($ Freq_index $) * \ldots$ 


$$
\exp (-\mathrm{j} * 2 * \text { pi } * \text { Freq }(\text { Freq_index }) * \text { taw })
$$

end

$\% \mathrm{H}$ should be symmetric for negative and positive freq $\mathrm{H}($ end $:-1$ : end-length $($ Freq $) / 2+1)=\mathrm{H}(1: 1: \operatorname{length}($ Freq $) / 2)$;

pow_H $=1 /\left(\mathrm{N}^{\wedge} 2\right) * \operatorname{sum}(\mathrm{H} . * \operatorname{conj}(\mathrm{H}))$;

$\%$ Transmitter side

$\%$ noise power is $-174 \mathrm{dBm} / \mathrm{Hz}$

Noise_pow_lin $=1 / 2 * 10^{\wedge}(-174 / 10) * 1 \mathrm{e}-3 * \mathrm{BW}$

for Eb_index $=1$ : length (SNR_dB_vect $)$

SNR_dB $=\mathrm{SNR}_{-} \mathrm{dB} \mathrm{S}_{-} \operatorname{vect}($ Eb_index $)$;

$\operatorname{disp}\left(\left[\right.\right.$ 'SNR_dB $=$ ', num2str $\left.\left.\left(\mathrm{SNR} \_\mathrm{dB}_{\mathrm{N}}\right)\right]\right)$;

$\%$ modify Trans power based on SNR of the channel

$\%$ snr_lin $=10^{\wedge}\left(\mathrm{Eb}_{-} \mathrm{N}_{-} \mathrm{dB} / 10\right)$;

SNR_lin $=10^{\wedge}\left(\mathrm{SNR} \_\mathrm{dB} / 10\right)$;

pow_Rx_lin $=\mathrm{SNR}_{-} \mathrm{lin} * \mathrm{Noise}_{\text {_pow_lin }}$

pow_Tx_lin $=$ pow_Rx_lin/pow_H;

\%amp of the Tx based on SNR

amp_Tx $=$ sqrt $($ pow_Tx_lin $)$;

$\mathrm{Tx}=\mathrm{x} \cdot *$ amp_Tx;

$\operatorname{freq}_{-} \mathrm{fs}=\mathrm{fft}(\mathrm{Tx}, \mathrm{N})$

\%modulate the sliced fs pulse based on RF signal modulated_fs $=\mathrm{AOM}($ phase, Freq,freq_fs, filter_step ); 
\% Channel effect on the fs signal

$\mathrm{RX}_{-}$sig_nonoise $=$modulated_fs.$* H ;$

\% Calculating Bit Error Rate-

$\mathrm{BER}=0 ;$

for iteration $=1$ : iteration_no

\% Generated noise is added to the signal

$R_{\text {X_sig }}=$ RX_sig_nonoise $+\ldots$

$(1 / \operatorname{sqrt}(2) *$ sqrt (Noise_pow_lin $) * \ldots$

complex (randn(size (RX_sig_nonoise $)), \ldots$

randn(size (RX_sig_nonoise )) ));

$\%$ Reciever side

equalized_sig_freq = RX_sig./ H;

rec_bits $=$ bit_Det (Freq, equalized_sig_freq,$\ldots$

bit_no, freq_fs, band_no, ...

freq_filter,filter_step );

for ind $=1$ : bit_no

if $\left(\right.$ rec_bits $_{-}$ind $) \sim$ trans_bits (ind $\left.)\right)$

$\mathrm{BER}=\mathrm{BER}+1 ;$

end

end 
end

BER_vect $\left(\mathrm{rh}_{-}\right.$ind, Eb_index $)=\ldots$

$\mathrm{BER} /($ length ( trans_bits $) *$ iteration_no $)$;

end

end

end

$\%$

$\%$ Function for detecting the bits in the receiver $\%$

function rec_bits $=$ bit_Det(Freq, modulated_fs , ...

bit_no, spectral_fs, band_no, freq_filter, filter_step )

$\mathrm{N}=\operatorname{length}($ Freq $)$;

$\%$ shift the recieved modulated_fs by one wave length

shifted_mod_fs $=\operatorname{zeros}(1, N)$;

for index $=1:$ round $(\mathrm{N} / 2)$

shifted_mod_fs(index+filter_step $)=$ modulated_fs(index );

end

\%copy the first half of the signal into rest

shifted_mod_fs $($ end: -1 : end-round $(\mathrm{N} / 2))=\ldots$

shifted_mod_fs $(1: \operatorname{round}(\mathrm{N} / 2)+1)$;

$\%$ Slice the recieved modulated_fs and

$\%$ the shifted fs in filter_size steps

filtered_mod_fs $=$ zeros(band_no, $\mathrm{N})$; 
filtered_shifted_fs $=$ zeros(band_no $-1, N)$;

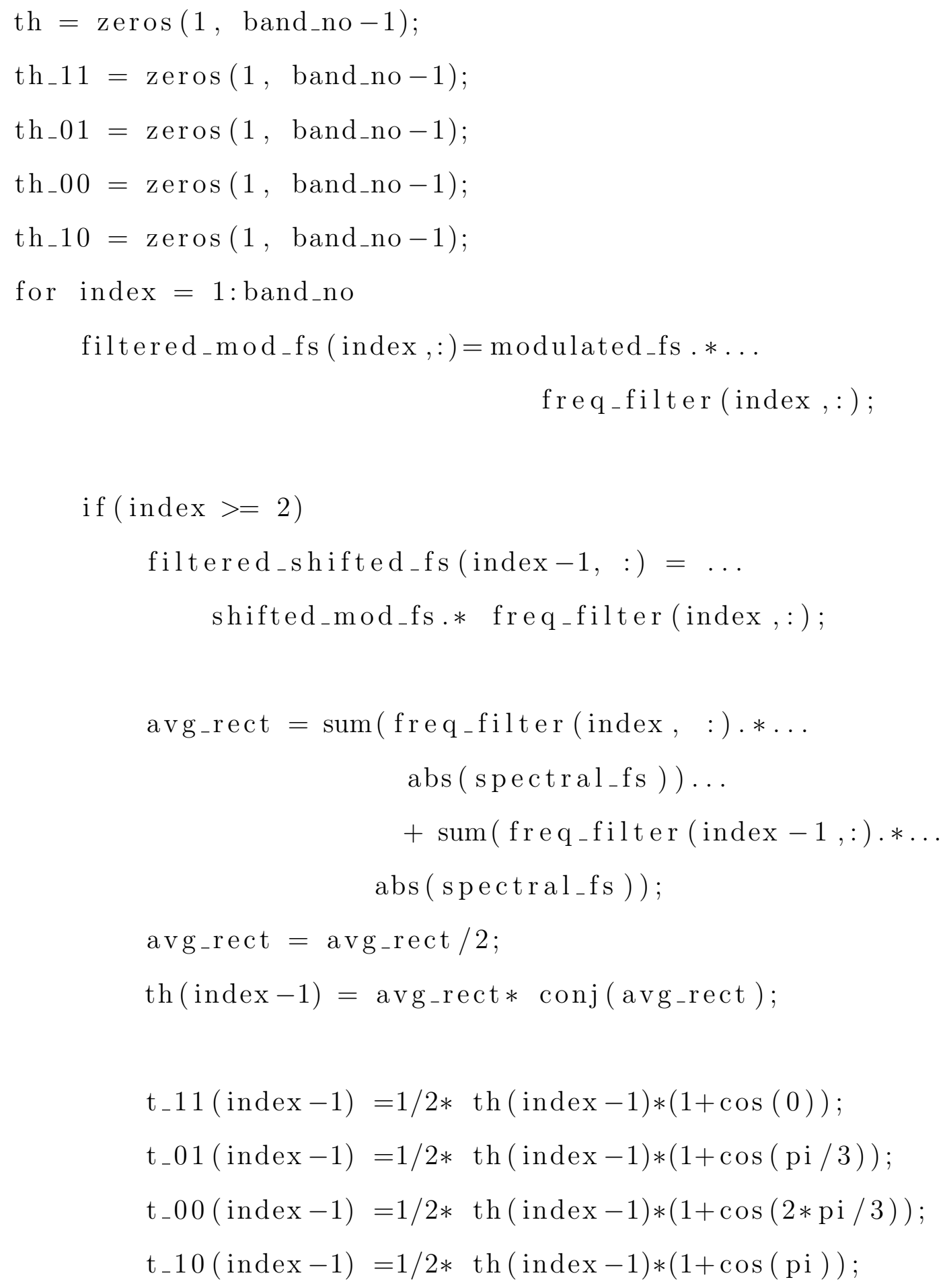




$$
\begin{aligned}
\operatorname{th}_{-} 11(\operatorname{index}-1) & =\left(t_{-} 11(\operatorname{index}-1)+t_{-} 01(\operatorname{index}-1)\right) / 2 \\
t_{-} 01(\operatorname{index}-1) & =\left(t_{-} 01(\operatorname{index}-1)+t_{-} 00(\operatorname{index}-1)\right) / 2 \\
t_{h} 00(\operatorname{index}-1) & =\left(t_{-} 00(\operatorname{index}-1)+t_{-} 10(\operatorname{index}-1)\right) / 2
\end{aligned}
$$

end

end

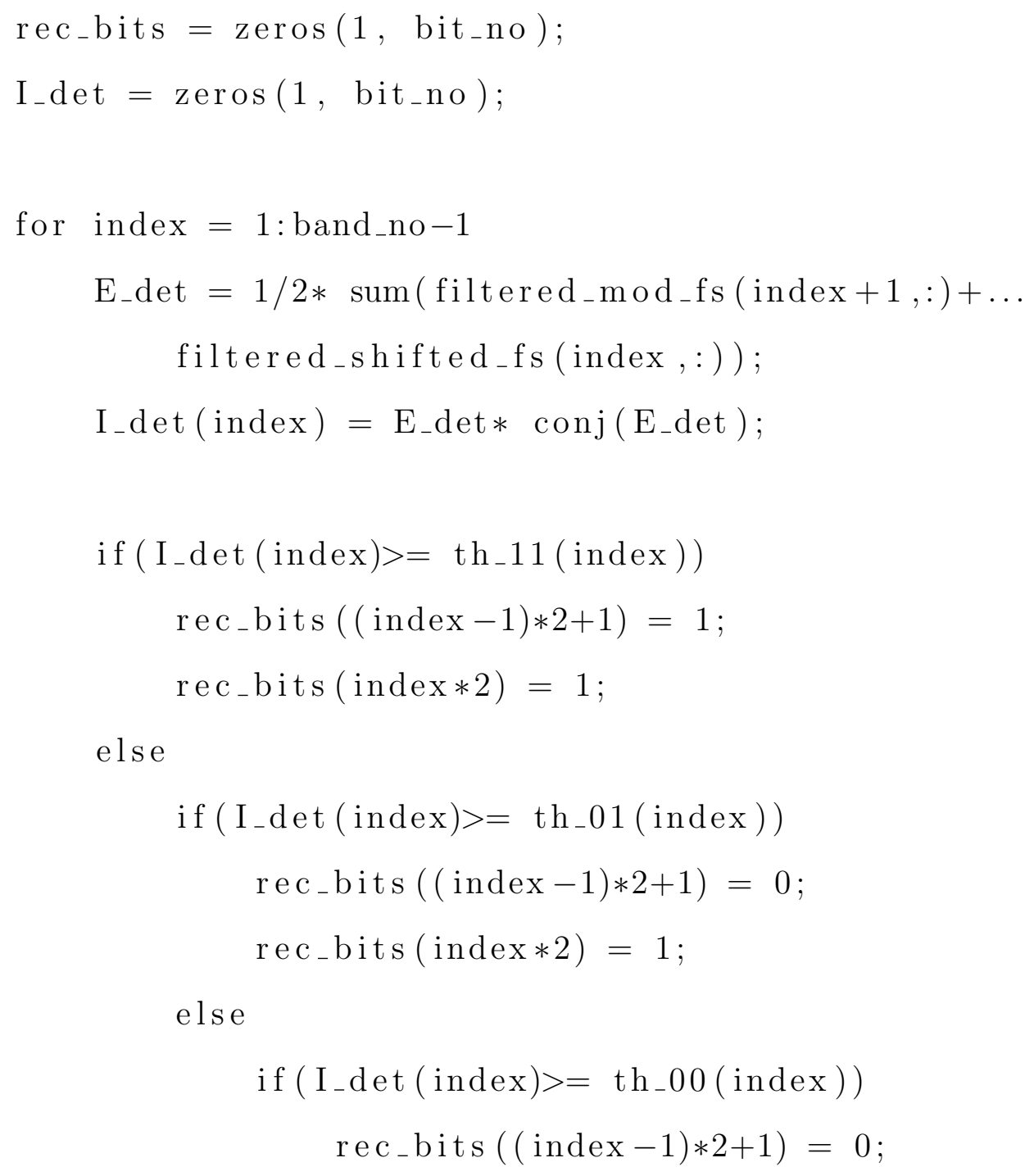


rec_bits $(\operatorname{index} * 2)=0$;

else

rec_bits $(($ index -1$) * 2+1)=1$;

rec_bits $(\operatorname{index} * 2)=0$;

end

end

end

end

end

$\%$

$\%$ transfer the input bits into phase information

$\%$ a (i ): the original bits

$\% \mathrm{~b}(1)=0 ; \mathrm{b}(\mathrm{i})=(\mathrm{a}(\mathrm{i})+\mathrm{b}(\mathrm{i}-1)) \bmod 2 ;$

$\%$ phase $=$ pi $* b(\mathrm{i})$

$\%$

function phase $=$ DQPSK_phase(trans_bits)

band_no $=\operatorname{length}\left(\operatorname{trans} \_\right.$bits $) / 2+1$;

phase $=$ zeros $(1$, band_no $)$;

$\operatorname{phase}(1)=0$;

for ind $=2$ : band_no

phase_ref $=$ phase_ref_func $($ trans_bits $(($ ind -2$) * 2+1), \ldots$

trans_bits $(($ ind -1$) * 2))$;

phase $($ ind $)=$ phase $($ ind -1$)+$ phase_ref;

if $(\operatorname{abs}(\operatorname{phase}($ ind $)-2 *$ pi $)<=0.01)$ 


$$
\begin{aligned}
& \text { phase }(\text { ind })=0 ; \\
& \text { elseif }(\text { phase }(\text { ind })>2 * \text { pi }) \\
& \text { phase }(\text { ind })=\text { phase }(\text { ind })-(2 * \text { pi }) \\
& \text { end }
\end{aligned}
$$

end

end

$\%$

\% Function for calculating the reference phase $\%$

function phase_ref $=$ phase_ref_func(bit1, bit2)

$$
\begin{aligned}
& \text { if (bit } 1=1 \& \& \text { bit } 2=1 \text { ) } \\
& \text { phase_ref }=0 \text {; } \\
& \text { else } \\
& \text { if (bit } 1=1 \& \& \text { bit } 2=0 \text { ) } \\
& \text { phase_ref }=\text { pi; } \\
& \text { else } \\
& \text { if (bit } 1=0 \text { \&\& bit } 2=1 \text { ) } \\
& \text { phase_ref }=\text { pi } / 3 \text {; } \\
& \text { els e } \\
& \text { if ( bit } 1==0 \text { \&\& bit } 2=0 \text { ) } \\
& \text { phase_ref }=2 * \text { pi } / 3 ; \\
& \text { end }
\end{aligned}
$$

end 
end

end

end

$\%$

$\%$ phase modulate the spectral signal:

$\%$ phase modulate sliced fs_signal (spectral spectrum)

$\%$ with the $\operatorname{rf}($ phase info):

$\% \exp (\mathrm{j} * \operatorname{phase}(\mathrm{i})) * \operatorname{rect}(\mathrm{w}-\mathrm{w}(\mathrm{i}), \quad$ delta_w $)$

$\%$

function modulated_fs $=\operatorname{AOM}($ phase, Freq, fs_sig, filter_step $)$

$\mathrm{N}=\operatorname{length}($ Freq $)$;

modulated_fs $=f_{\text {fs_sig }}$

for index $=1: \operatorname{length}($ phase $)$

Firstind $=($ index -1$) *$ filter_step +1 ;

LastInd $=$ FirstInd+filter_step -1 ;

modulated_fs $($ FirstInd: LastInd $)=\ldots$

$\exp (\mathrm{j} * \operatorname{phase}(\mathrm{index})) . * \ldots$

$\operatorname{abs}($ fs_sig (FirstInd : LastInd ) );

end

modulated_fs $($ end $:-1: \operatorname{end}-\operatorname{round}(\mathrm{N} / 2))=\ldots$

modulated_fs $(1: \operatorname{round}(\mathrm{N} / 2)+1) ;$

end 
Appendix E

NS3:THZ PROPAGATION LOSS MODEL C++ HEADER FILE

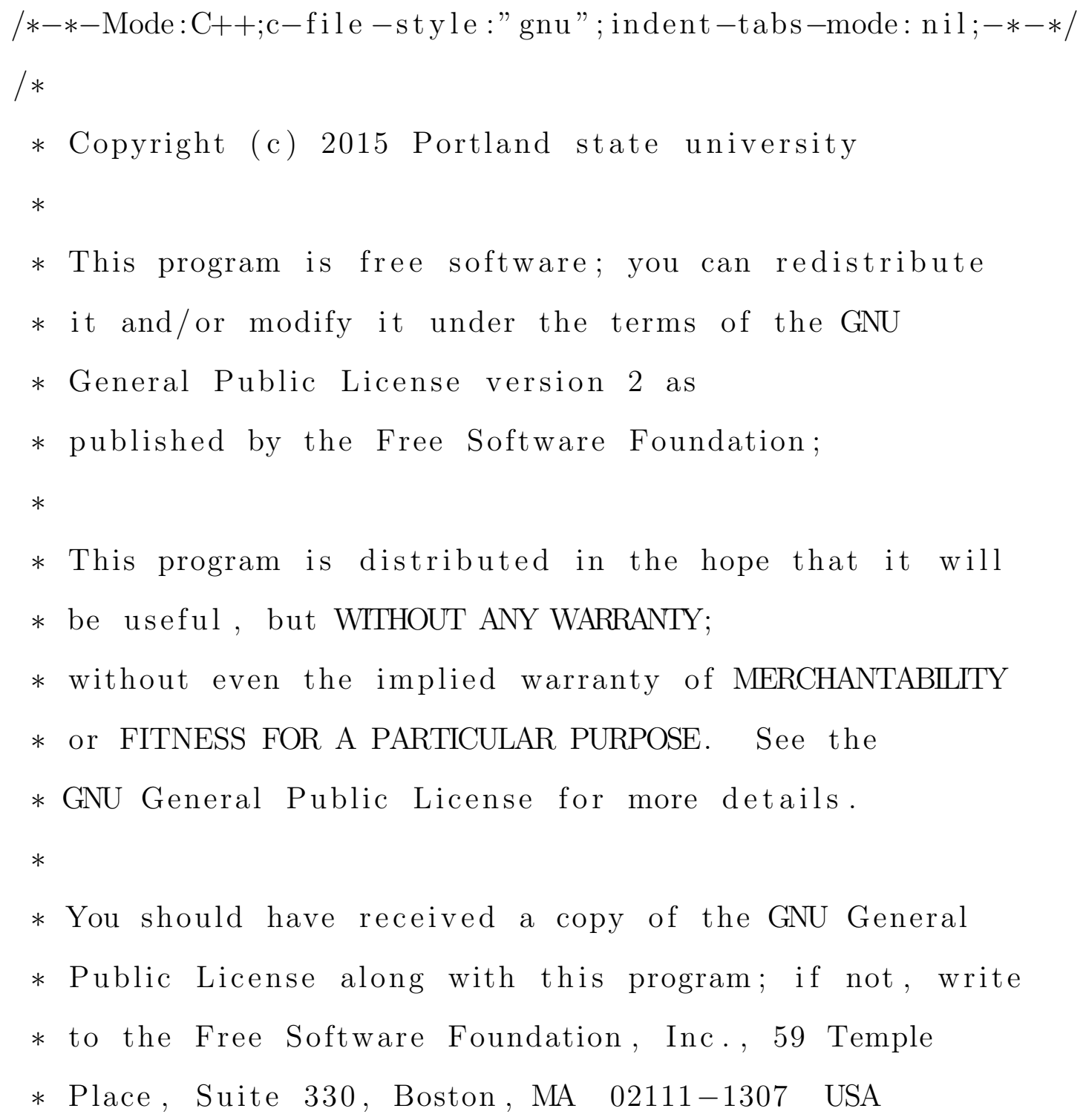




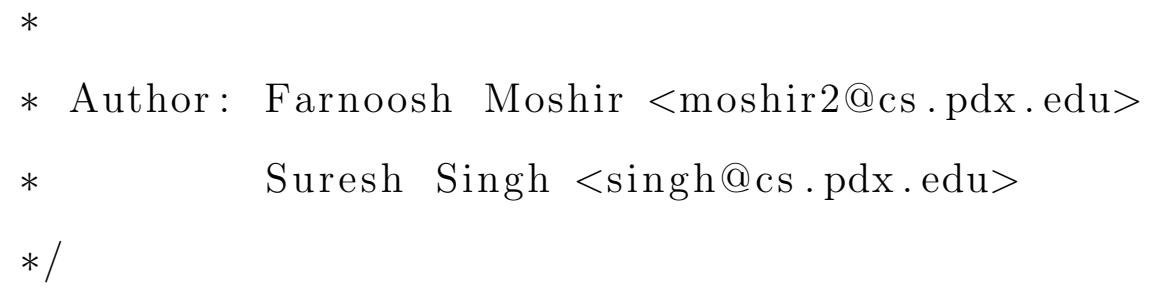


* - $\backslash \mathrm{f} \$$ P_r $\backslash \mathrm{f} \$$ : reception power $(\mathrm{W})$

* - $\backslash \mathrm{f} \$ \mathrm{P}_{-} \mathrm{t} \backslash \mathrm{f} \$$ : transmission power (W)

* $-\backslash \mathrm{f} \$ \mathrm{G}_{-} \mathrm{t} \backslash \mathrm{f} \$$ : transmission gain (unit-less)

* $-\backslash \mathrm{f} \$$ G_r $\backslash \mathrm{f} \$$ : reception gain (unit-less)

* $-\backslash \mathrm{f} \$ \mathrm{~d} \backslash \mathrm{f} \$:$ distance $(\mathrm{m})$

* $-\backslash \mathrm{f} \$ \mathrm{~K}(\mathrm{f}, \mathrm{h}) \backslash \mathrm{f} \$$ : Attenuation coefficient of

* frequency $\mathrm{f}$ and humidity $\mathrm{h}$

* $-\backslash \mathrm{f} \$ \mathrm{~L} \backslash \mathrm{f} \$$ : system loss (unit-less)

$*$

* In the implementation, \f\$ \lambda \f\$ is calculated as

$* \backslash \mathrm{f} \$ \backslash$ frac $\{\mathrm{C}\}\{\mathrm{f}\} \backslash \mathrm{f} \$$, where $\backslash \mathrm{f} \$ \mathrm{C}=299792458 \backslash \mathrm{f} \$ \mathrm{~m} / \mathrm{s}$ is

* the speed of light in

* vacuum, and $\backslash \mathrm{f} \$ \mathrm{f} \backslash \mathrm{f} \$$ is the frequency in $\mathrm{Hz}$ which can

* be configured by

* the user via the Frequency attribute.

$*$

* This model is used for the cases where Tx and Rx both

* use collimated lenses. As a

* result, the signal has the minimum divergence. However,

* in $\mathrm{THz}$ domain atmospheric

* molecules cause signal attenuation.

$*$

$* \backslash f \$ K(f, h) \$ \backslash f$ is the attenuation coefficient for frequency

$* \backslash \mathrm{f} \$ \mathrm{f} \backslash \mathrm{f} \$$ and relative humidity $\backslash \mathrm{f} \$ \mathrm{~h} \backslash \mathrm{f} \$$ and corresponds

* to attenuation resulting from water vapor, oxygen,

* nitrogen, and carbon dioxide. $\backslash \mathrm{f} \$ \mathrm{~K}(\mathrm{f}, \mathrm{h}) \mathbb{\$} \backslash \mathrm{f}$ is found 
* based on Van Vleck-Weisskopt (VW) line shape model

* for frequencies up to $2 \mathrm{THz}$.

* The required parameters have been derived from

* HITRAN database.

$*$

* This calculated atmospheric attenuation coefficient

* is given in file "absorptionTable.txt". The file has three

* columns. The first Column corresponds to

* $0 \%$ relative humidity. The second column corresponds

* to $40 \%$, and the third column corresponds to $80 \%$

* relative humidity.

$*$

* There are 5121 rows in the file. Each row corresponds

* to a specific frequency $(\mathrm{Hz})$ in the range of $1 \mathrm{~Hz}$ to

* $2 \mathrm{THz}$ frequency.

$*$

* variable m_freqLastInd corresponds to the total number

* of rows in the file and variable m_freqEnd corresponds

* to the highest frequency $(\mathrm{Hz})$ that is used in this model.

$* /$

class ThzDirectionalPropagationLossModel:

public PropagationLossModel

\{

public:

static TypeId GetTypeId (void); 


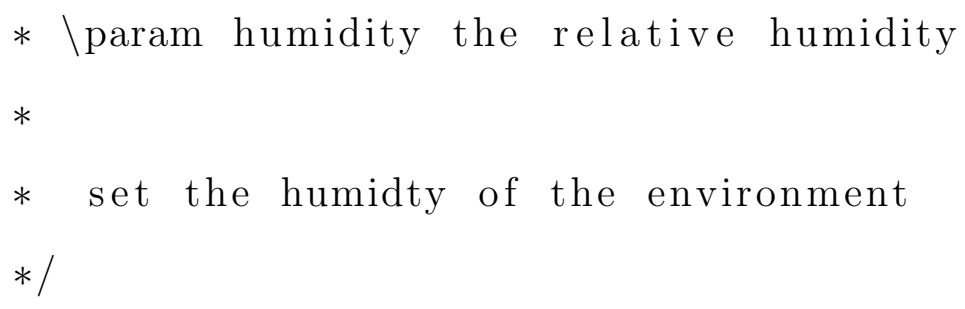

void SetHumidity (uint32_t humidity);

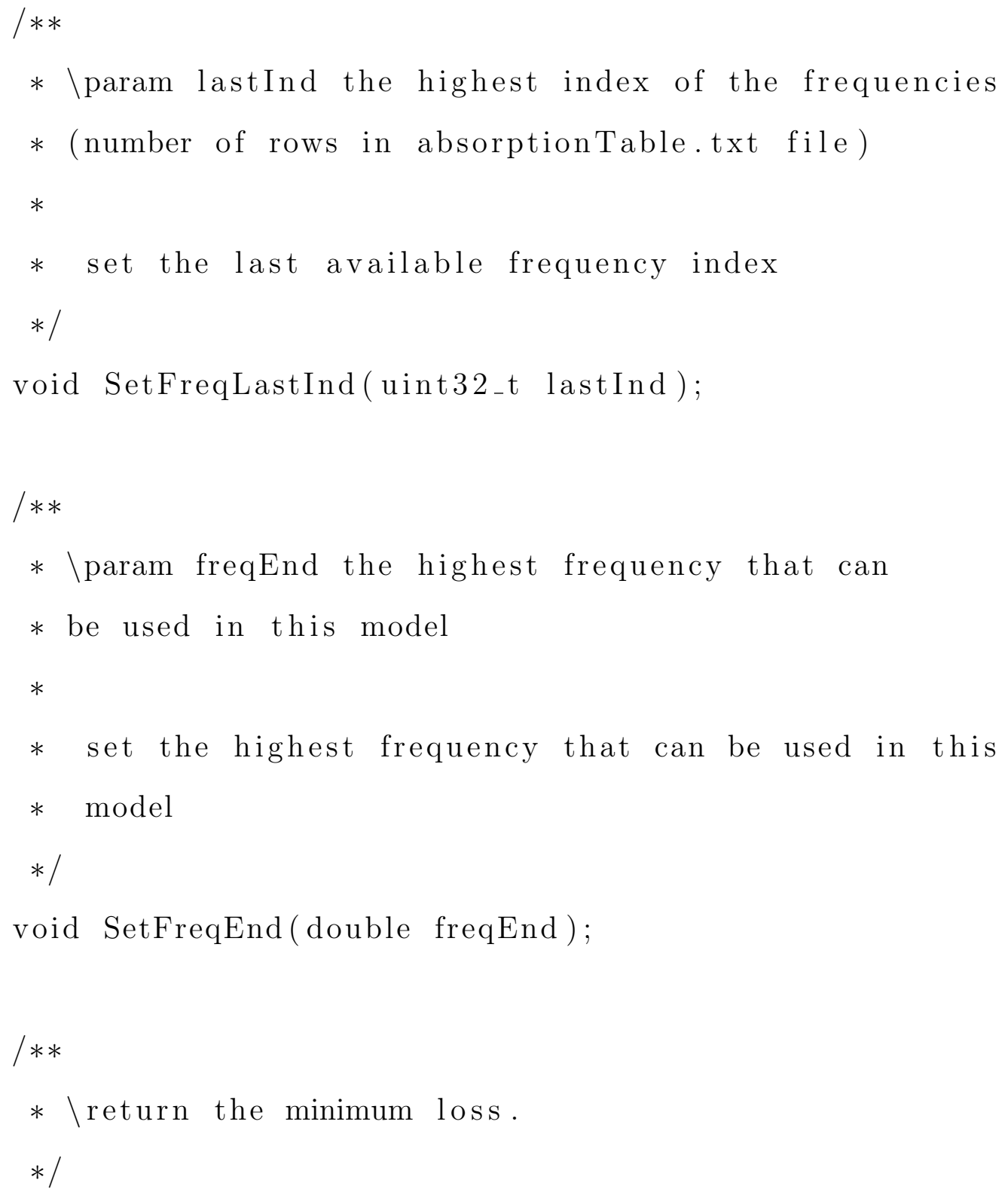




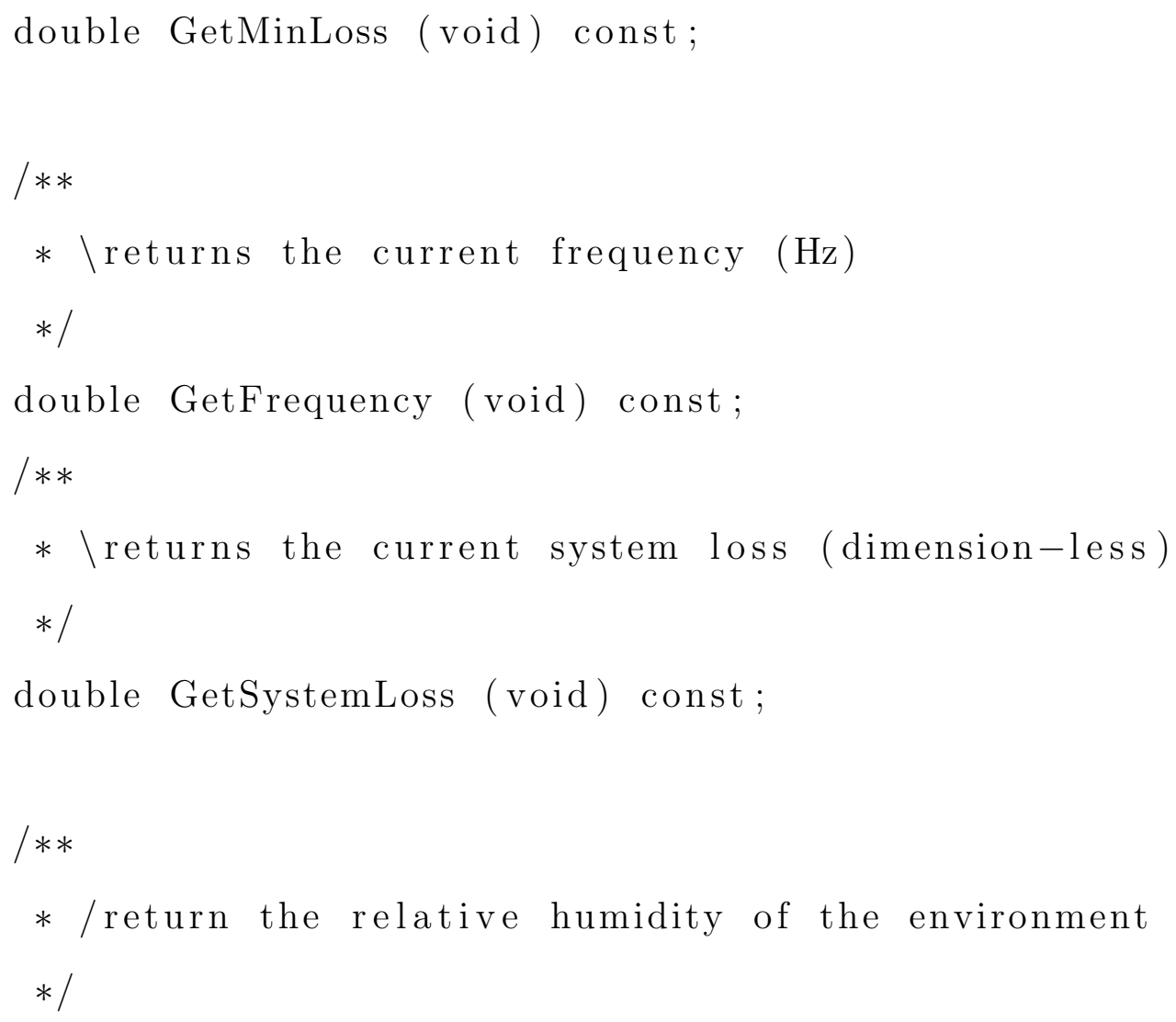


double GetFreqEnd (void) const;

private:

ThzDirectionalPropagationLoss Model

(const ThzDirectionalPropagationLossModel \&o);

ThzDirectionalPropagationLossModel \& operator =

(const ThzDirectionalPropagationLossModel \&o);

virtual double DoCalcRxPower (double txPowerDbm,

$$
\text { Ptr }<\text { MobilityModel }>\text { a }
$$$$
\text { Ptr }<\text { MobilityModel }>\text { b) const ; }
$$

virtual int64_t DoAssignStreams (int64_t stream);

double DbmToW (double dbm) const;

double DbmFromW (double w) const;

double FindAttenuationCoefficient () const;

double m_lambda;

double m_frequency;

double m_systemLoss ;

double m_minLoss ;

uint $32_{-}$t m_humidity;

uint 32 _t m_freqLastInd ;

double m_freqEnd;

\} 


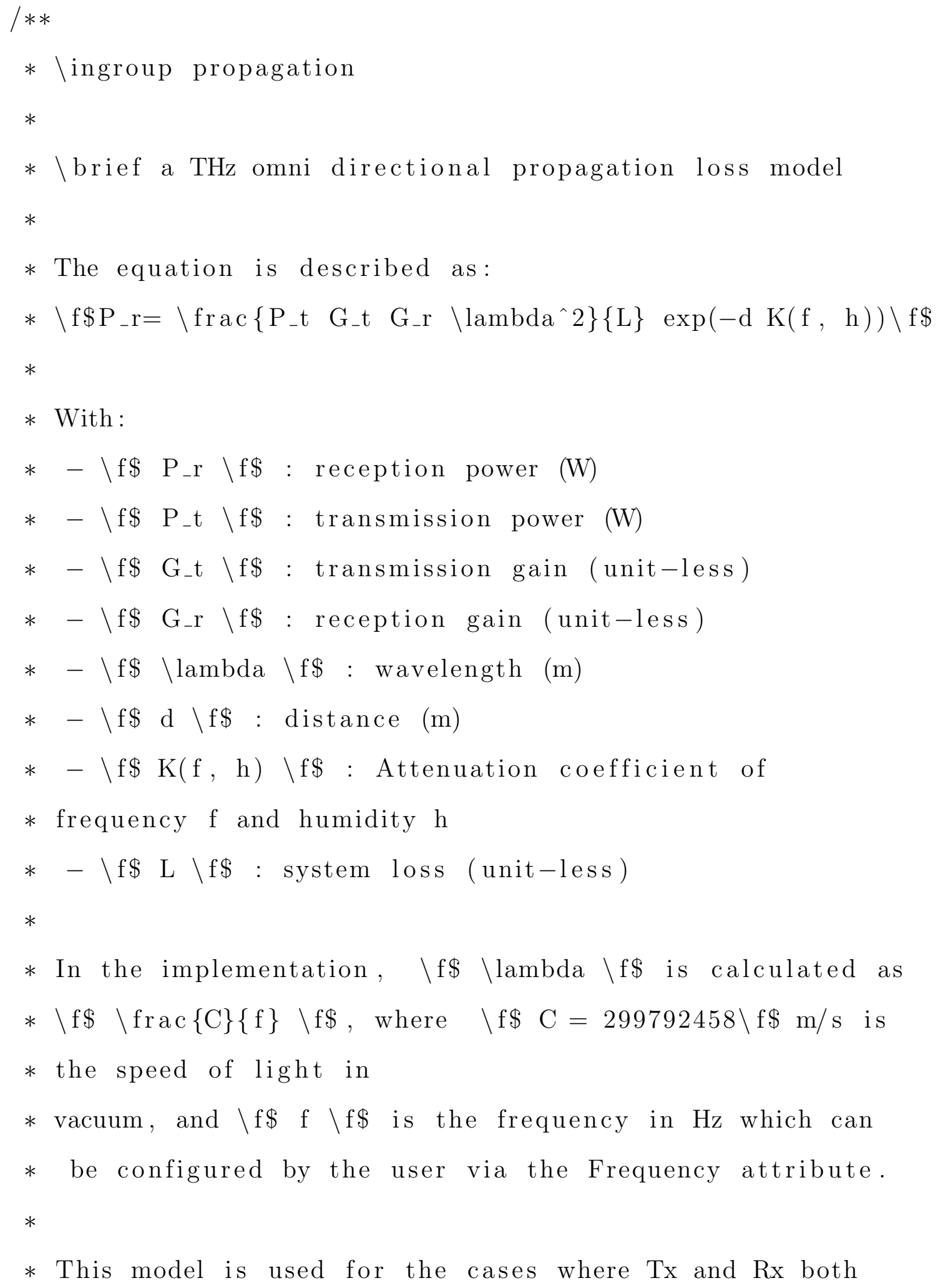


* use omni directional. Therefore, the free space path

* loss from Friis model and atmospheric attenuation

* resulting from molecules in the air should be considered.

$*$

$* \backslash \mathrm{f} \$ \mathrm{~K}(\mathrm{f}, \mathrm{h}) \$ \backslash \mathrm{f}$ is the attenuation coefficient for frequency

$* \backslash \mathrm{f} \$ \mathrm{f} \backslash \mathrm{f} \$$ and relative humidity $\backslash \mathrm{f} \$ \mathrm{~h} \backslash \mathrm{f} \$$ and corresponds

* to attenuation resulting from water vapor, oxygen,

* nitrogen, and carbon dioxide. $\backslash \mathrm{f} \$ \mathrm{~K}(\mathrm{f}, \mathrm{h}) \$ \backslash \mathrm{f}$ is found

* based on Van Vleck-Weisskopt (VW) line shape

* model for frequencies up to $2 \mathrm{THz}$ frequency.

* The required parameters have been derived from

* HITRAN database.

$*$

* This calculated atmospheric attenuation coefficient is

* given in file "absorptionTable.txt". The file has three

* columns. The first Column corresponds to $0 \%$ relative

* humidity. The second column corresponds to $40 \%$,

* and the third column corresponds to $80 \%$ relative humidity.

*

* There are 5121 rows in the file. Each row corresponds

* to a specific frequency $(\mathrm{Hz})$ in the range of $1 \mathrm{~Hz}$ to 2

* THz frequency.

$*$

* variable $m_{-}$freqLastInd corresponds to the total number

* of rows in the file and variable m_freqEnd corresponds

* to the highest frequency $(\mathrm{Hz})$ that is used in this model. 


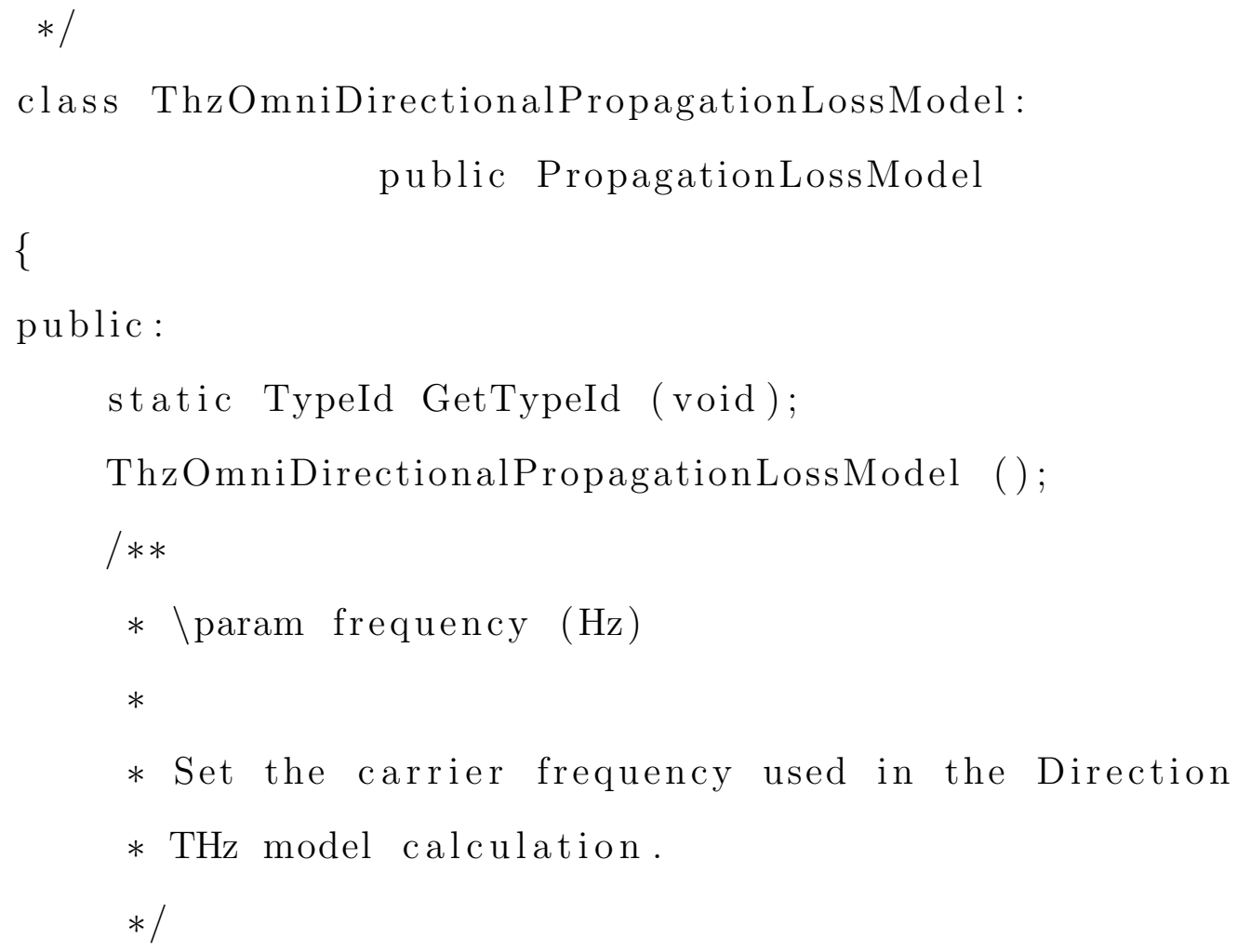


* propagation loss (in dB) will always be greater

* or equal than this value

$* /$

void SetMinLoss (double minLoss);

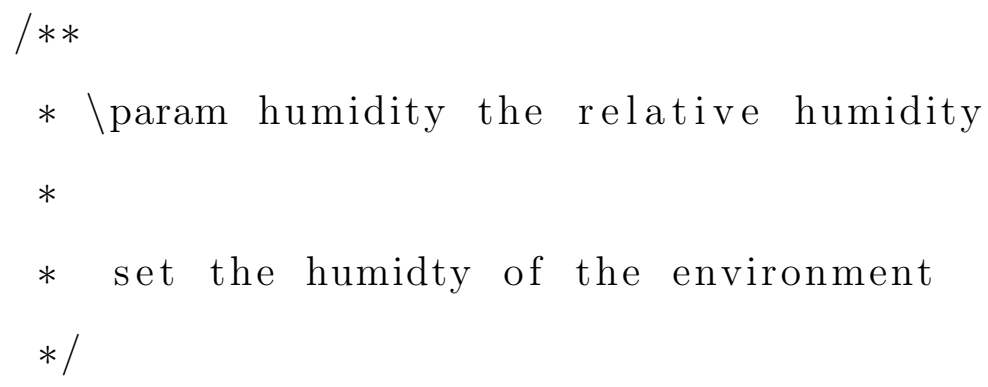




\section{$* /$}

void SetFreqEnd(double freqEnd);

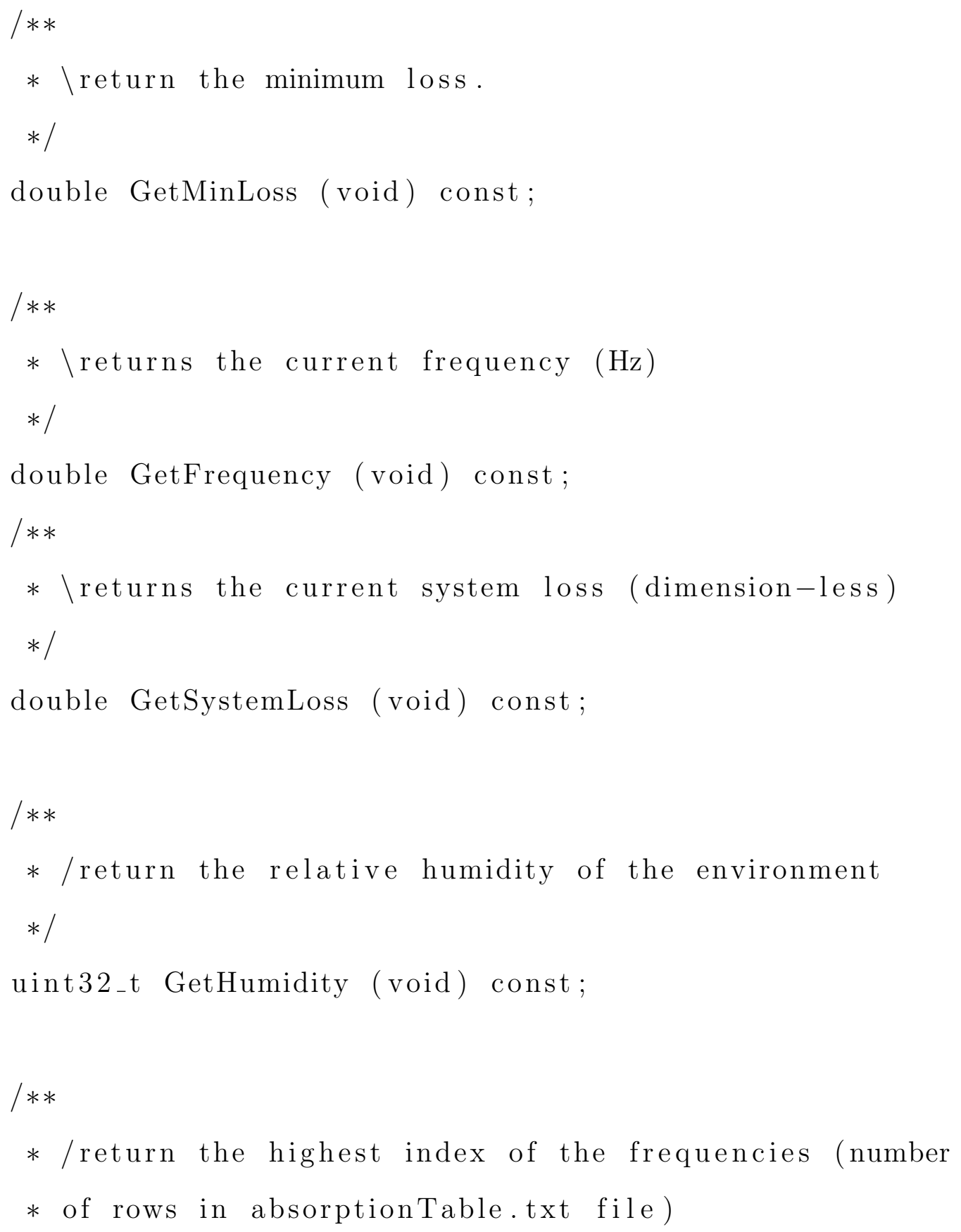




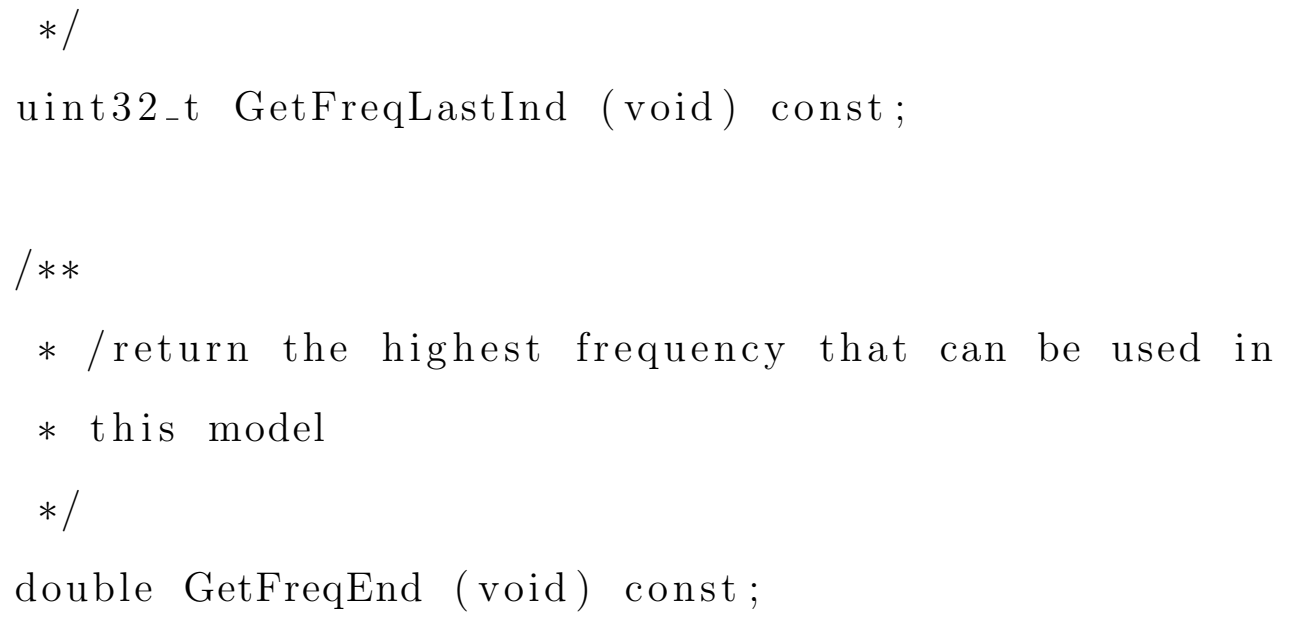

private:

ThzOmniDirectionalPropagationLoss Model ( const

ThzOmniDirectionalPropagationLossModel \&o );

ThzOmniDirectionalPropagationLossModel \& operator=

(const ThzOmniDirectionalPropagationLossModel \&o ) ;

virtual double DoCalcRxPower (double txPowerDbm,

$$
\begin{aligned}
& \operatorname{Ptr}<\text { MobilityModel }>\text { a }, \\
& \operatorname{Ptr}<\text { MobilityModel }>\text { b) const } ;
\end{aligned}
$$

virtual int64_t DoAssignStreams (int64_t stream);

double DbmToW (double dbm) const;

double DbmFromW (double w) const;

double FindAttenuationCoefficient () const;

static const double PI;

double m_lambda;

double m_frequency; 
double m_systemLoss ;

double m_minLoss;

uint $32_{-}$t m_humidity ;

uint $32_{-} \mathrm{t}$ m_freqLastInd ;

double m_freqEnd;

\}

\} // namespace ns3

\#endif $/ *$ THZPROPAGATIONLOSS_MODEL_H */ 
Appendix F

NS3: THZ PROPAGATION LOSS MODEL C++ SOURCE CODE

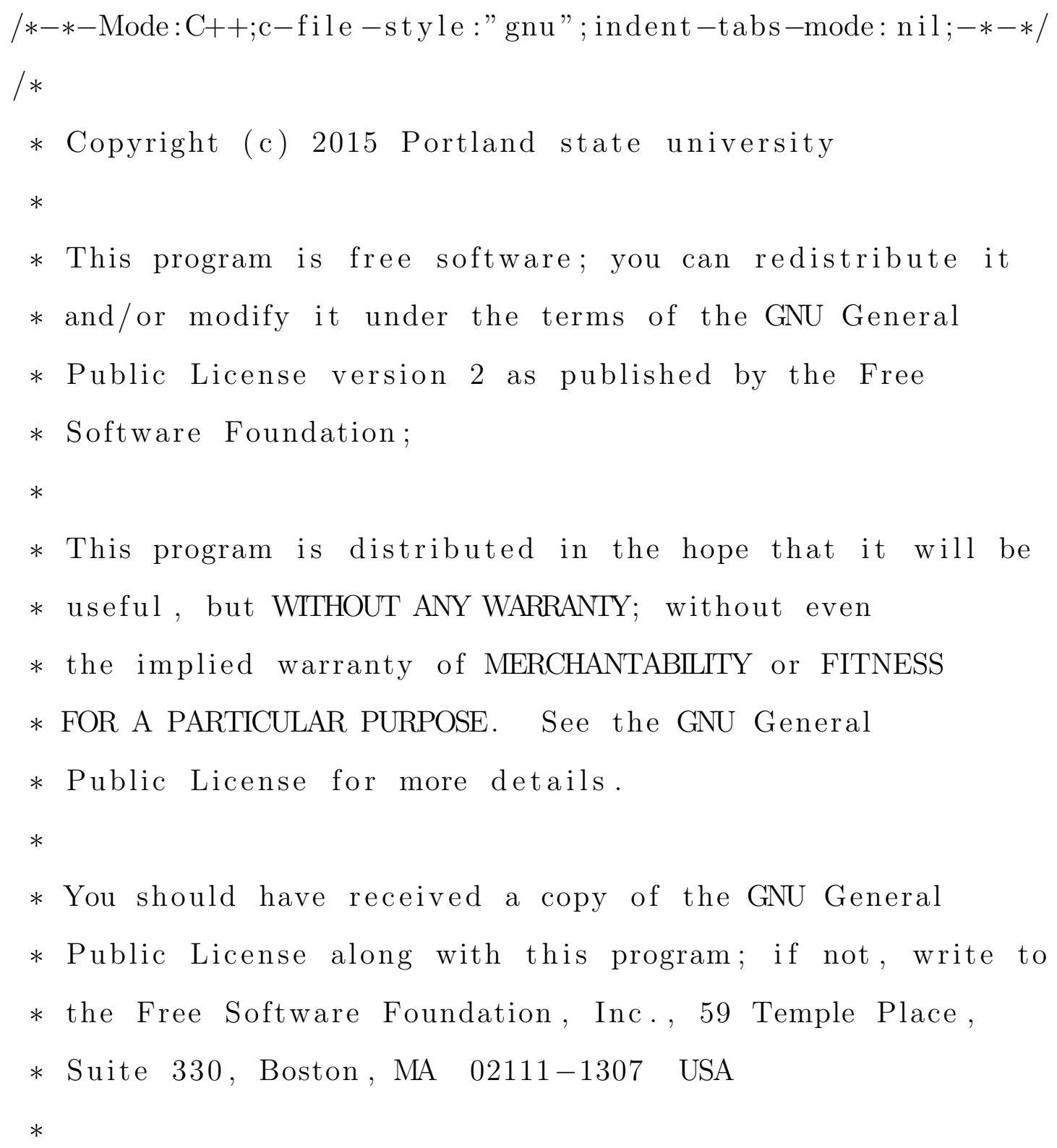


* Author: Farnoosh Moshir <moshir2@cs.pdx.edu>

* $\quad$ Suresh Singh $<$ singh@cs . pdx.edu>

$* /$

\#include "thz-propagation-loss-model.h"

\#include "ns3/log.h"

\#include "ns3/mobility-model.h"

\#include "ns3/double.h"

\#include "ns3/uinteger.h"

\#include "ns3/string.h"

\#include "ns3/pointer.h"

\#include <cmath $>$

\#include $<$ fstream $>$

NSLOG_COMPONENTDEFINE ("ThzPropagationLossModel");

namespace ns3 \{

NS_OBJECT_ENSURE_REGISTERED(

ThzDirectionalPropagationLossModel );

TypeId

ThzDirectionalPropagationLoss Model : : GetTypeId (void)

\{

static TypeId tid = TypeId

("ns3 : ThzDirectionalPropagationLossModel")

. SetParent $<$ PropagationLossModel $>()$ 
. AddConstructor $<$ ThzDirectionalPropagationLossModel $>$ () . AddAttribute ("Frequency",

"The carrier frequency (in $\mathrm{Hz}$ ) at which propagation occurs (default is $237.5 \mathrm{GHz}) . "$, DoubleValue (237.5 e9), MakeDoubleAccessor ( \&ThzDirectionalPropagationLoss Model : :

SetFrequency, \&ThzDirectionalPropagationLoss Model : :

GetFrequency),

MakeDoubleChecker $<$ double $>$ ( ))

. AddAttribute ("Humidity",

"The relative humidity (in \%)

of the channel (default is $40 \%$ )", UintegerValue (40),

MakeUintegerAccessor (

\&ThzDirectionalPropagationLoss Model : : SetHumidity, \&ThzDirectionalPropagationLossModel : : GetHumidity),

MakeUintegerChecker $<$ uint32_t $>\quad())$

.AddAttribute ("SystemLoss", "The system loss", DoubleValue (1.0), MakeDoubleAccessor (\&ThzDirectionalPropagationLossModel : : m_systemLoss ), 


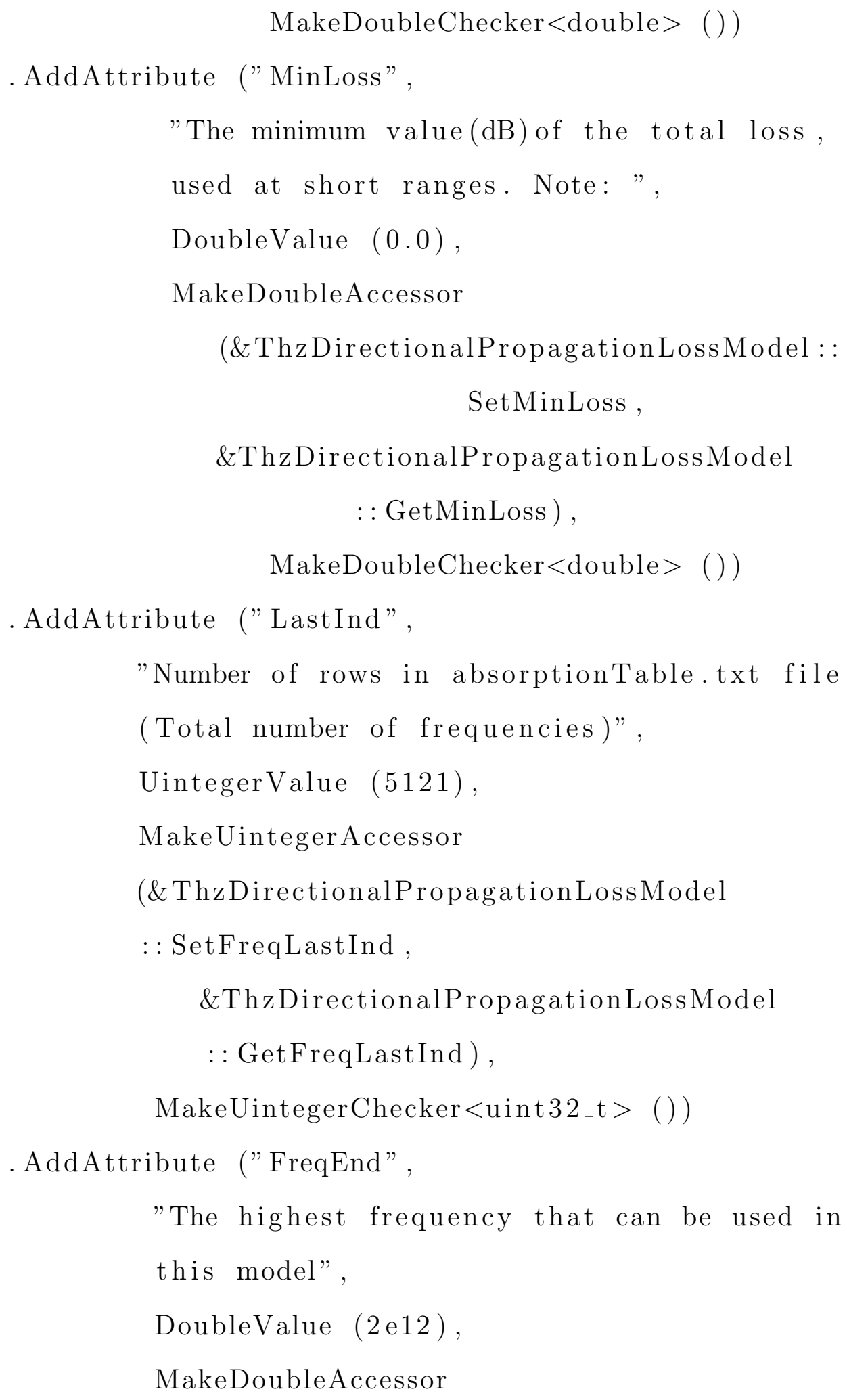


(\&ThzDirectionalPropagationLoss Model ::

SetFreqEnd, \&ThzDirectionalPropagationLoss Model

: : GetFreqEnd ),

MakeDoubleChecker $<$ double $>())$

;

return tid;

\}

ThzDirectionalPropagationLoss Model : :

ThzDirectionalPropagationLossModel ()

\{

\}

void

ThzDirectionalPropagationLoss Model : :

SetSystemLoss (double systemLoss)

\{

$\mathrm{m}_{\text {_systemLoss }}=$ systemLoss;

\}

double

ThzDirectionalPropagationLoss Model : :

GetSystemLoss (void) const

\{

return m_systemLoss;

\}

void 
ThzDirectionalPropagationLoss Model : :

SetMinLoss (double minLoss)

\{

$\mathrm{m}_{\_} \operatorname{minLoss}=\operatorname{minLoss} ;$

\}

double

ThzDirectionalPropagationLoss Model : :

GetMinLoss (void) const

\{

return m_minLoss;

\}

void

ThzDirectionalPropagationLoss Model : :

SetFrequency (double frequency)

\{

$\mathrm{m}_{-}$frequency $=$frequency

static const double $\mathrm{C}=299792458.0$;

$\mathrm{m}_{-}$lambda $=\mathrm{C} /$ frequency;

\}

double

ThzDirectionalPropagationLoss Model : :

GetFrequency (void) const

\{

return $m_{-}$frequency; 


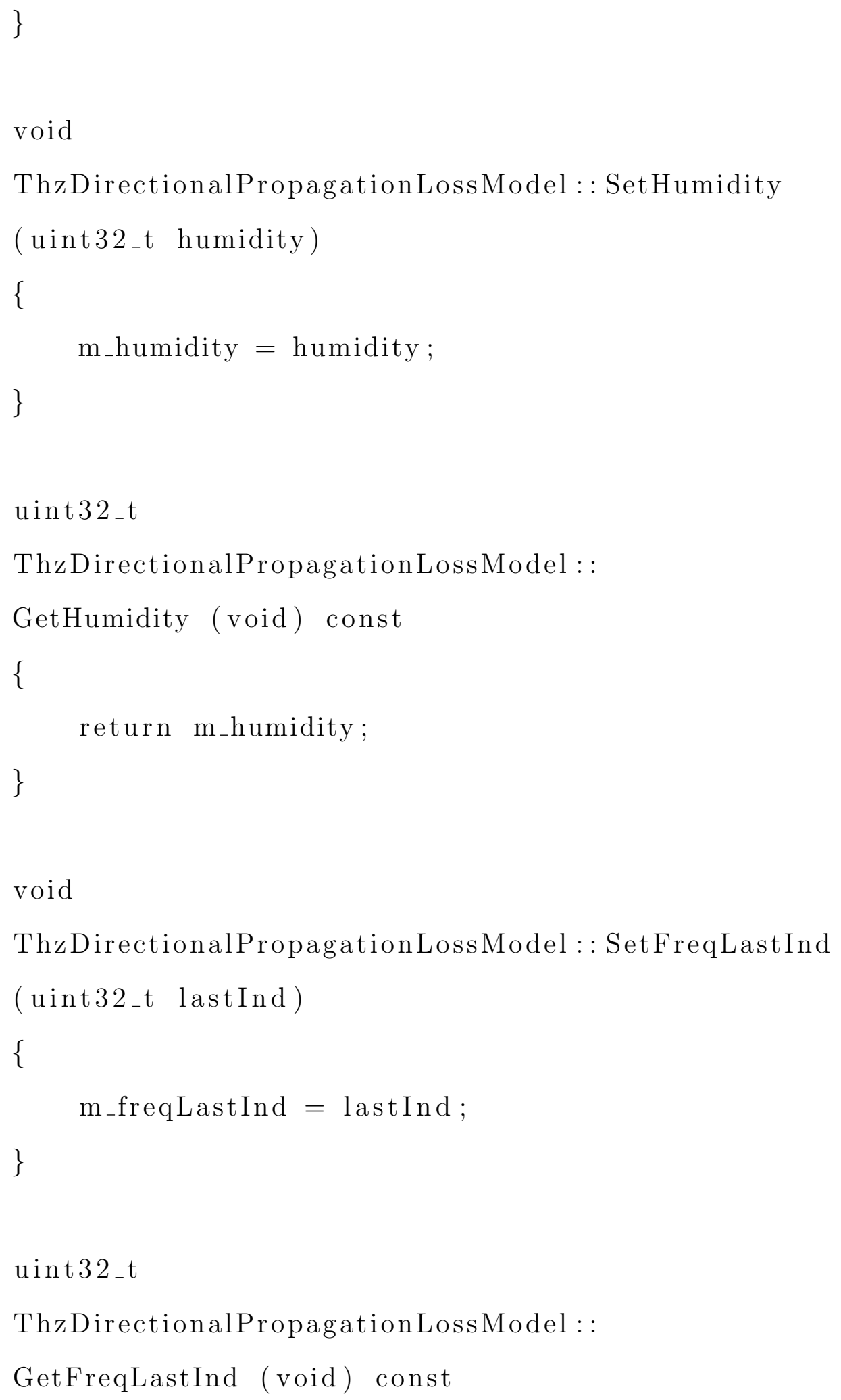




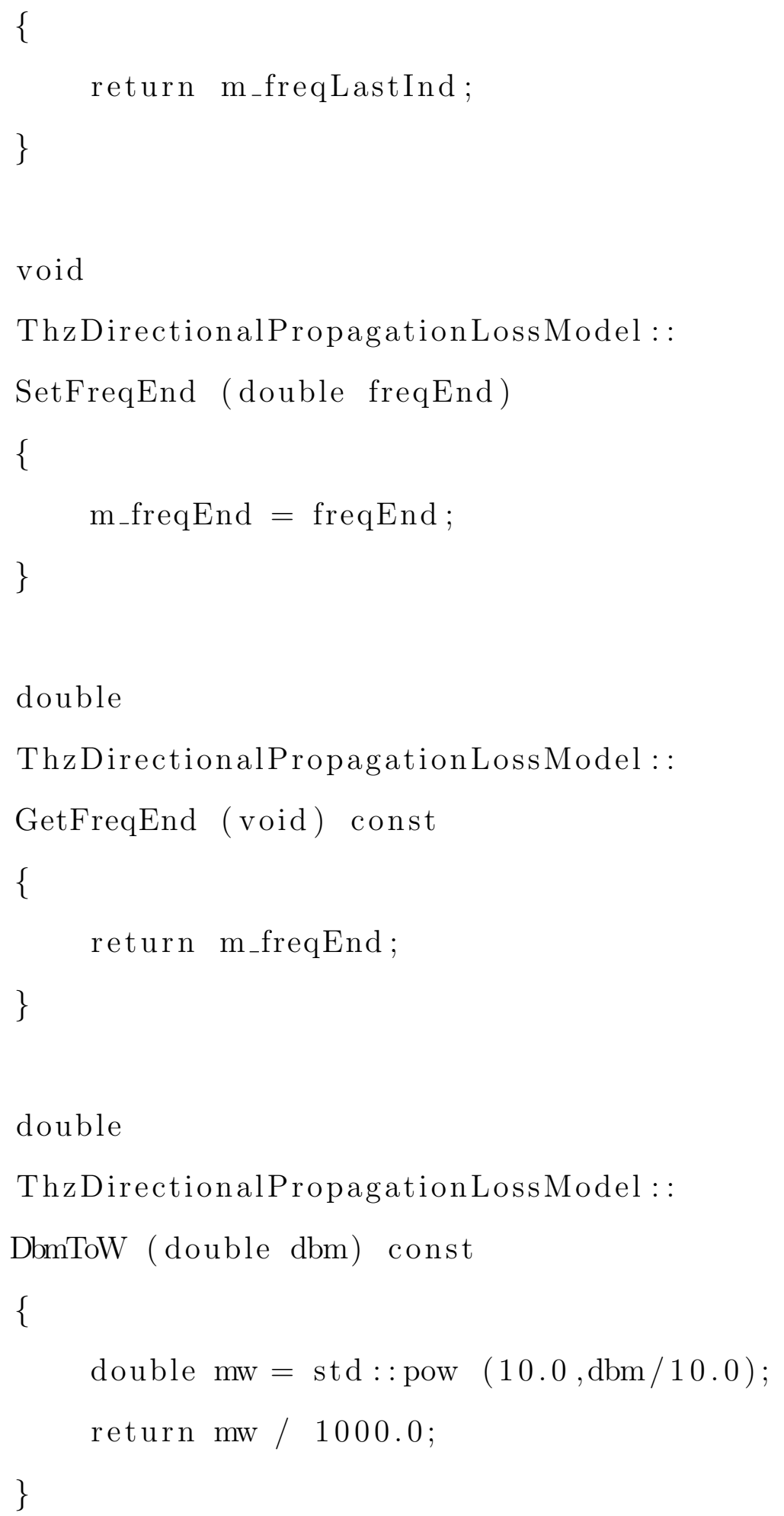


double

ThzDirectionalPropagationLoss Model : :

DbmFromW (double w) const

\{

double $\mathrm{dbm}=\operatorname{std}:: \log 10(\mathrm{w} * 1000.0) * 10.0 ;$

return dbm;

\}

double

ThzDirectionalPropagationLoss Model : :

DoCalcRxPower

(double txPowerDbm, Ptr $<$ MobilityModel $>$ a, $\operatorname{Ptr}<$ MobilityModel $>$ b) const

\{

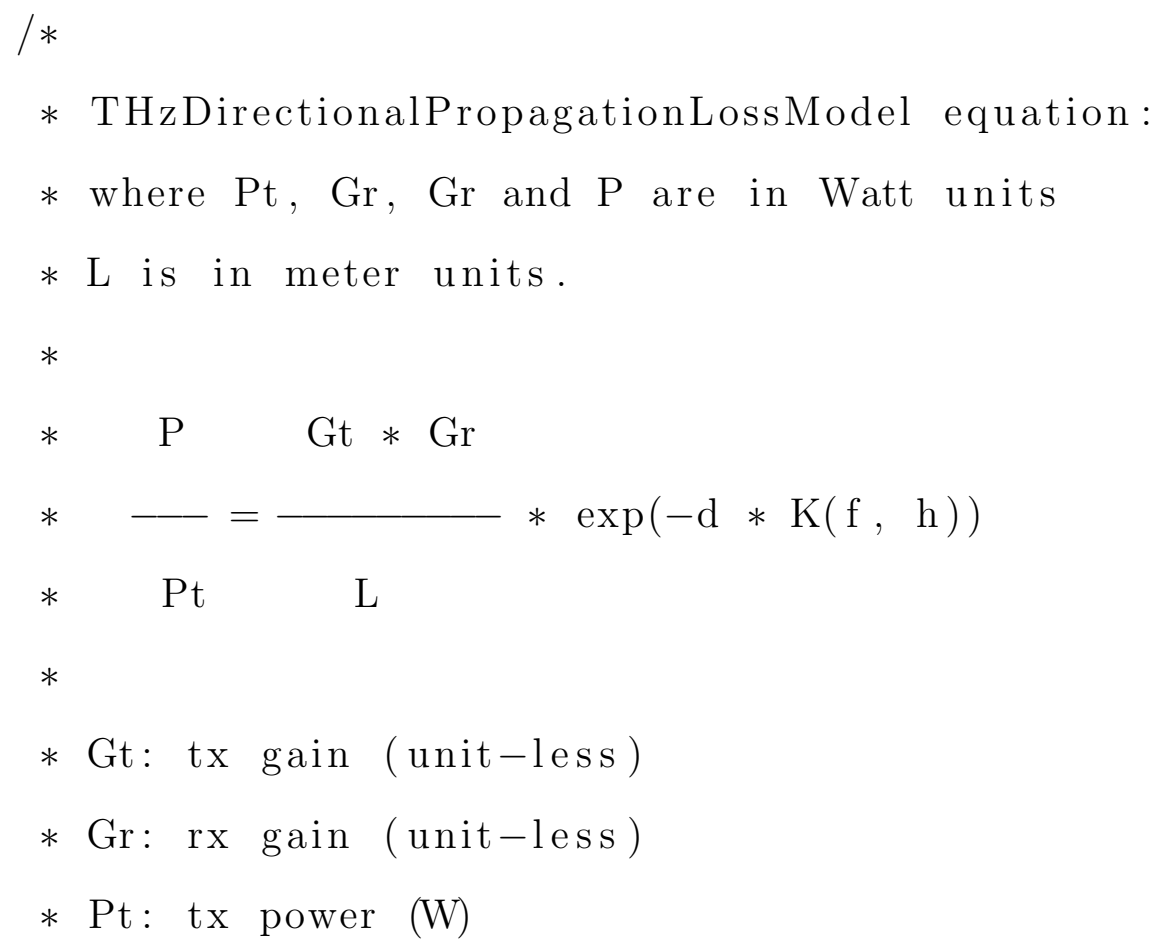


* d: distance $(\mathrm{m})$

* L: system loss

* f : frequency $(\mathrm{Hz})$

* $\mathrm{K}(\mathrm{f}, \mathrm{h})$ : Attenuation coefficient corresponds to

* frequency $\mathrm{f}$ and humidity $\mathrm{h}$

*

*

* Here, we ignore tx and rx gain and the input

* and output values are in $\mathrm{dB}$ or $\mathrm{dBm}$ :

*

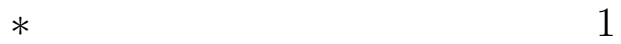

$* \mathrm{rx}=\mathrm{tx}+10 \log 10(---* \exp (-\mathrm{d} * \mathrm{~K}(\mathrm{f}, \mathrm{h})))$

$*$

L

*

* rx: rx power (dB)

* tx : tx power $(\mathrm{dB})$

* d: distance (m)

* L: system loss (unit-less)

* $K(f, h):$ Attenuation coefficient of frequency

* f and humidity h

$* /$

double distance $=a->$ GetDistanceFrom $(b)$;

if $($ distance $<0)$

\{

return txPowerDbm - m_minLoss;

\} 


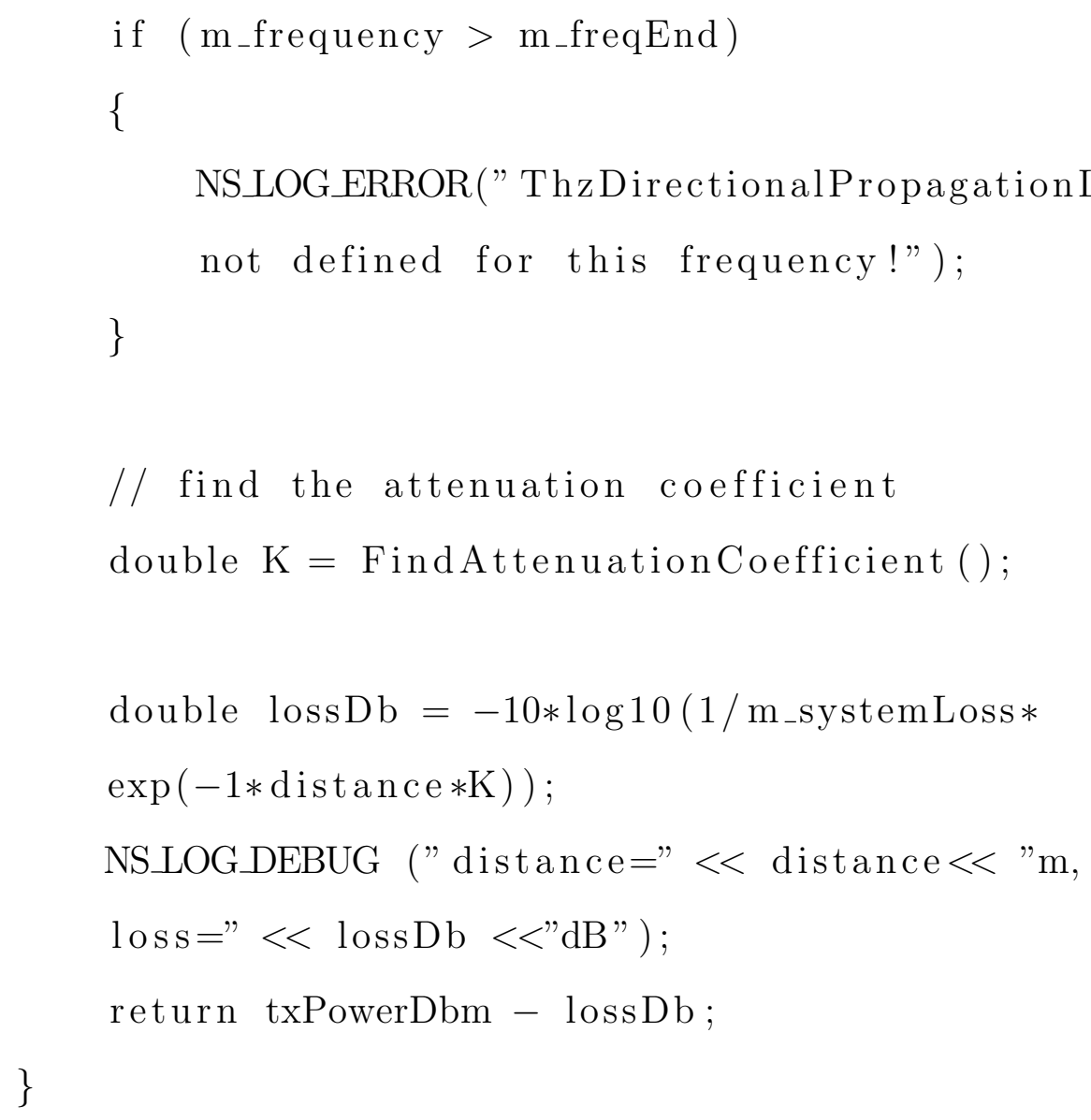




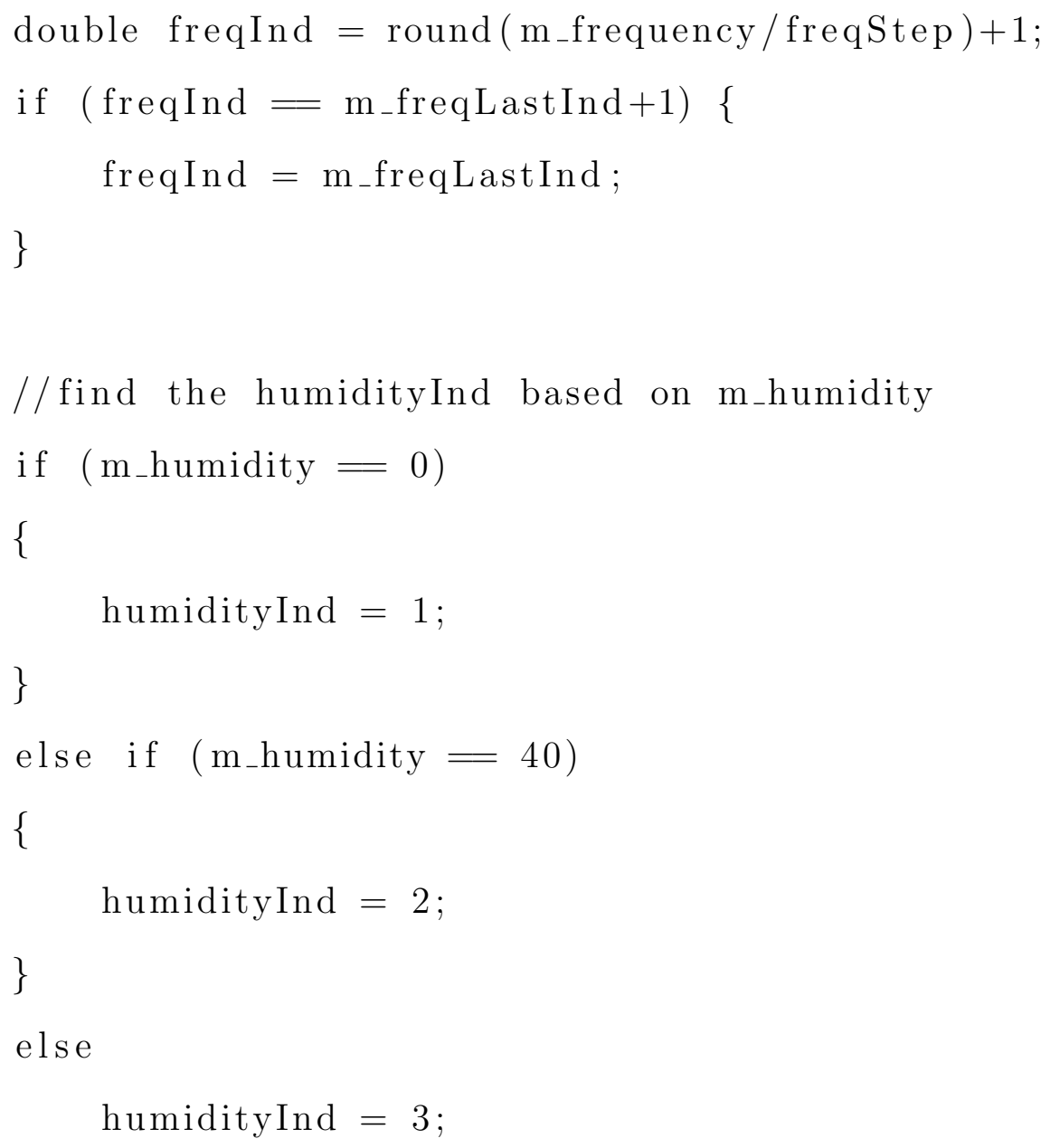




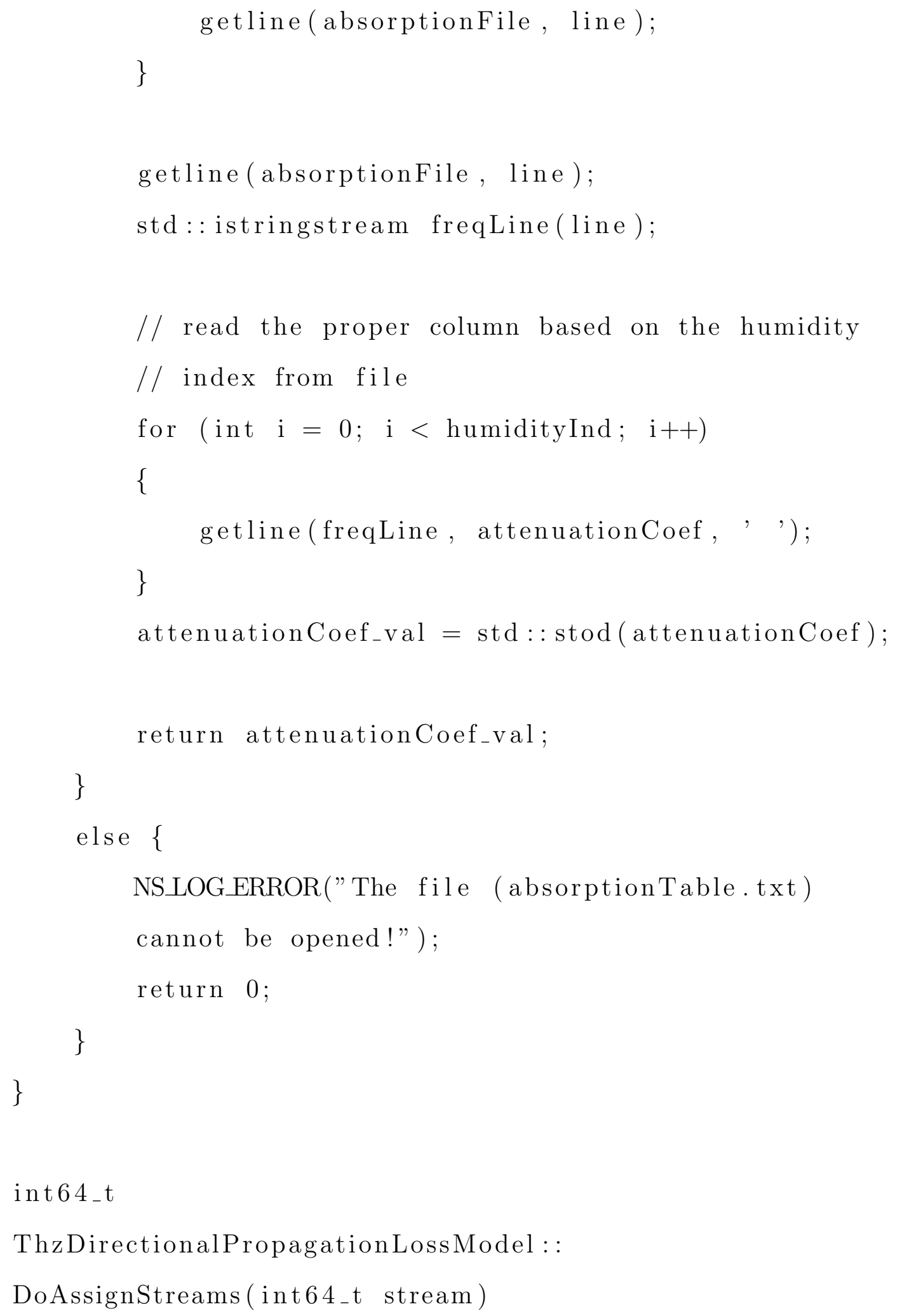


\}

$/ / 1 /$

\section{NS_OBJECT ENSURE_REGISTERED}

(ThzOmniDirectionalPropagationLossModel );

const double ThzOmniDirectionalPropagationLossModel $:: \mathrm{PI}=3.14159265358979323846$;

TypeId

ThzOmniDirectionalPropagationLossModel : GetTypeId (void) \{

static TypeId tid = TypeId ("ns3: :

ThzOmniDirectionalPropagationLossModel")

. SetParent $<$ PropagationLossModel $>\quad()$

. AddConstructor

$<$ ThzOmniDirectionalPropagationLossModel $>()$

. AddAttribute ("Frequency",

"The carrier frequency (in $\mathrm{Hz}$ ) at which propagation occurs (default is $237.5 \mathrm{GHz}$ )." , DoubleValue (237.5 e9), MakeDoubleAccessor 
(\&ThzOmniDirectionalPropagationLoss Model : : SetFrequency,

\&ThzOmniDirectionalPropagationLoss Model : :

GetFrequency ),

MakeDoubleChecker $<$ double $>($ ) $)$

.AddAttribute ("Humidity",

"The relative humidity (in \%) of the

channel (default is $40 \%$ )",

UintegerValue (40),

MakeUinteger Accessor

(\&ThzOmniDirectionalPropagationLoss Model : :

SetHumidity ,

\&ThzOmniDirectionalPropagationLoss Model : :

GetHumidity ),

MakeUintegerChecker $<$ uint32_t $>$ ())

.AddAttribute ("SystemLoss", "The system loss",

DoubleValue (1.0),

MakeDoubleAccessor

(\&ThzOmniDirectionalPropagationLoss Model : :

m_systemLoss ),

MakeDoubleChecker $<$ double $>$ ( ))

. AddAttribute ("MinLoss",

"The minimum value (dB) of the total loss, used at short ranges. Note: ",

DoubleValue (0.0),

MakeDoubleAccessor 
(\&ThzOmniDirectionalPropagationLoss Model ::

SetMinLoss,

\&ThzOmniDirectionalPropagationLossModel : :

GetMinLoss ),

MakeDoubleChecker $<$ double $>$ ( ) )

.AddAttribute ("FreqLastInd",

"Number of rows in absorptionTable.txt file

(Total number of frequencies)",

DoubleValue (5121),

MakeDoubleAccessor

(\&ThzOmniDirectionalPropagationLoss Model : :

SetFreqLastInd,

\&ThzOmniDirectionalPropagationLossModel : :

GetFreqLastInd ),

MakeDoubleChecker $<$ double $>())$

.AddAttribute ("FreqEnd",

"The highest frequency that can be used in this model",

DoubleValue (2 e12),

MakeDoubleAccessor

(\&ThzOmniDirectionalPropagationLoss Model : :

SetFreqEnd,

\&ThzOmniDirectionalPropagationLoss Model :

GetFreqEnd),

MakeDoubleChecker $<$ double $>$ ( ) ) 


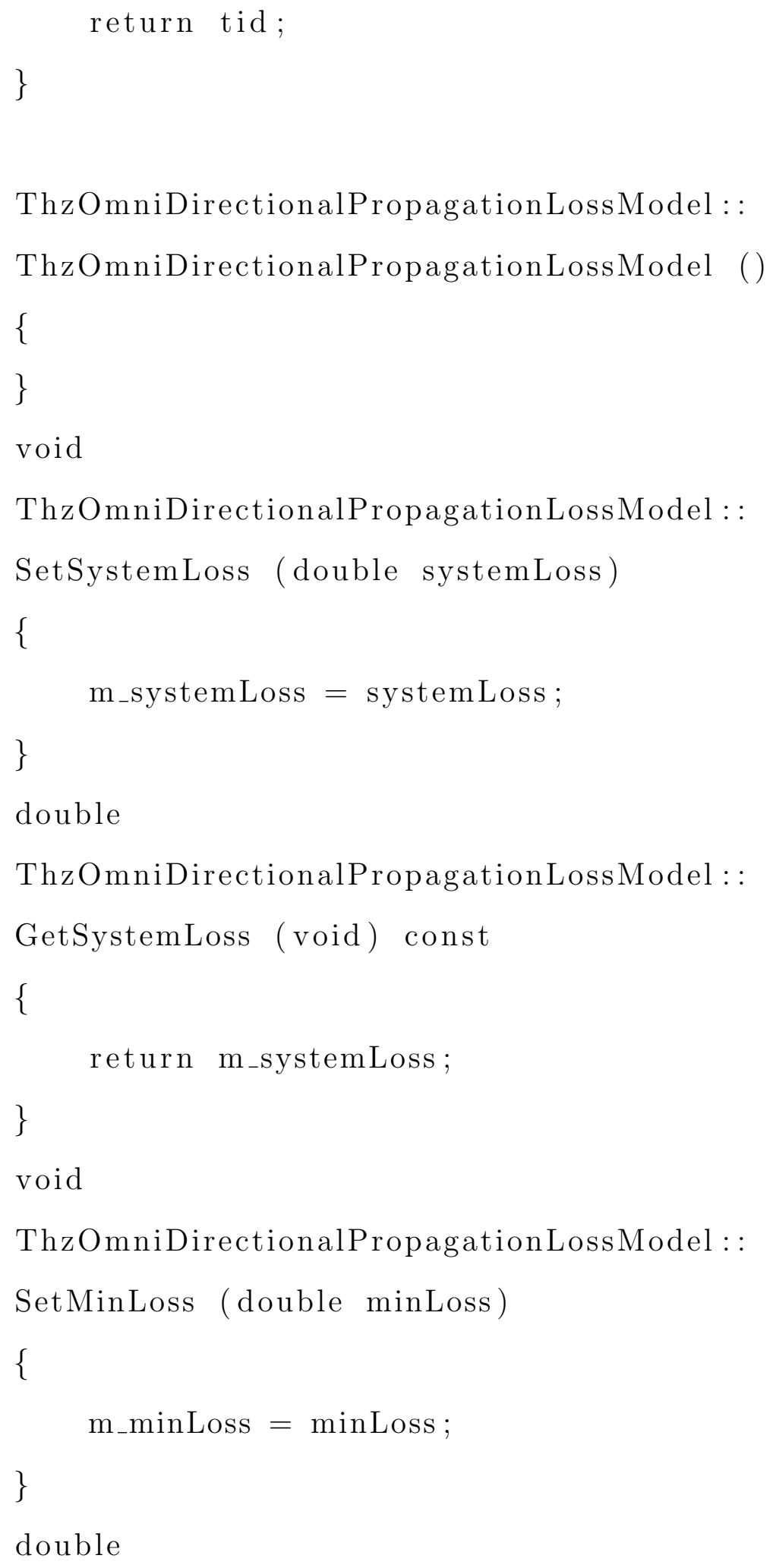


ThzOmniDirectionalPropagationLoss Model : :

GetMinLoss (void) const

\{

return m_minLoss;

\}

void

ThzOmniDirectionalPropagationLoss Model : :

SetFrequency (double frequency)

\{

$\mathrm{m}_{-}$frequency $=$frequency;

static const double $\mathrm{C}=299792458.0$;

$\mathrm{m}_{-}$lambda $=\mathrm{C} /$ frequency;

\}

double

ThzOmniDirectionalPropagationLoss Model : :

GetFrequency (void) const

\{

return m_frequency;

\}

void

ThzOmniDirectionalPropagationLoss Model : :

SetHumidity (uint32_t humidity) 


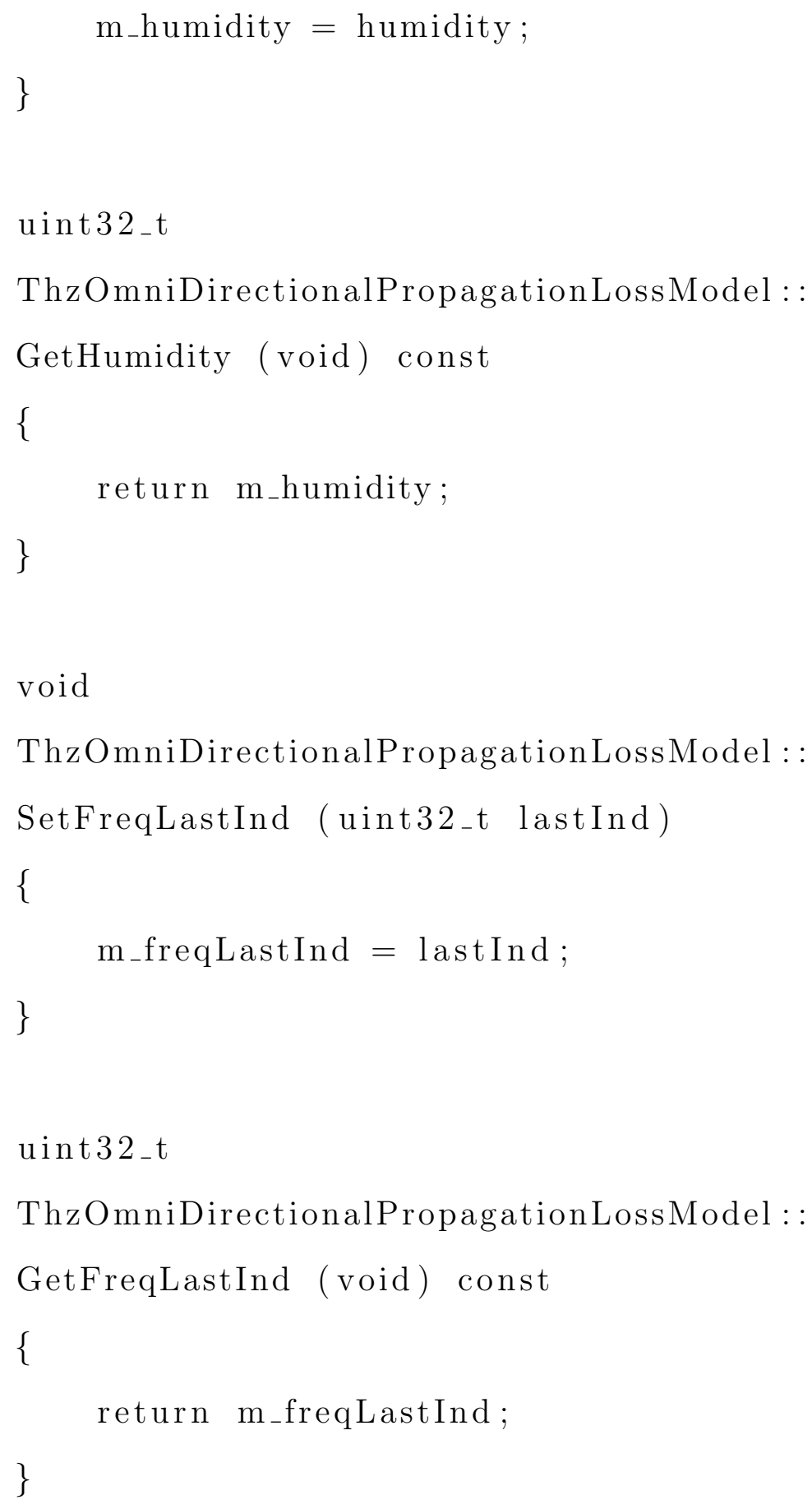




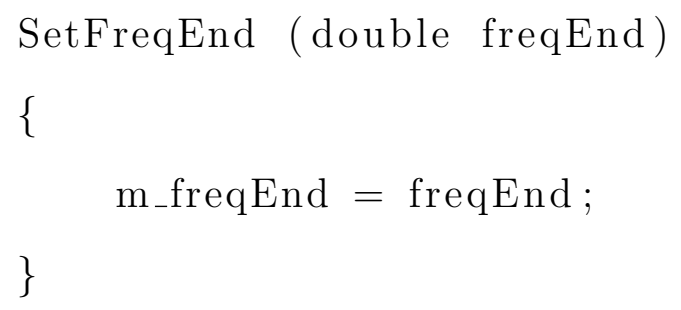




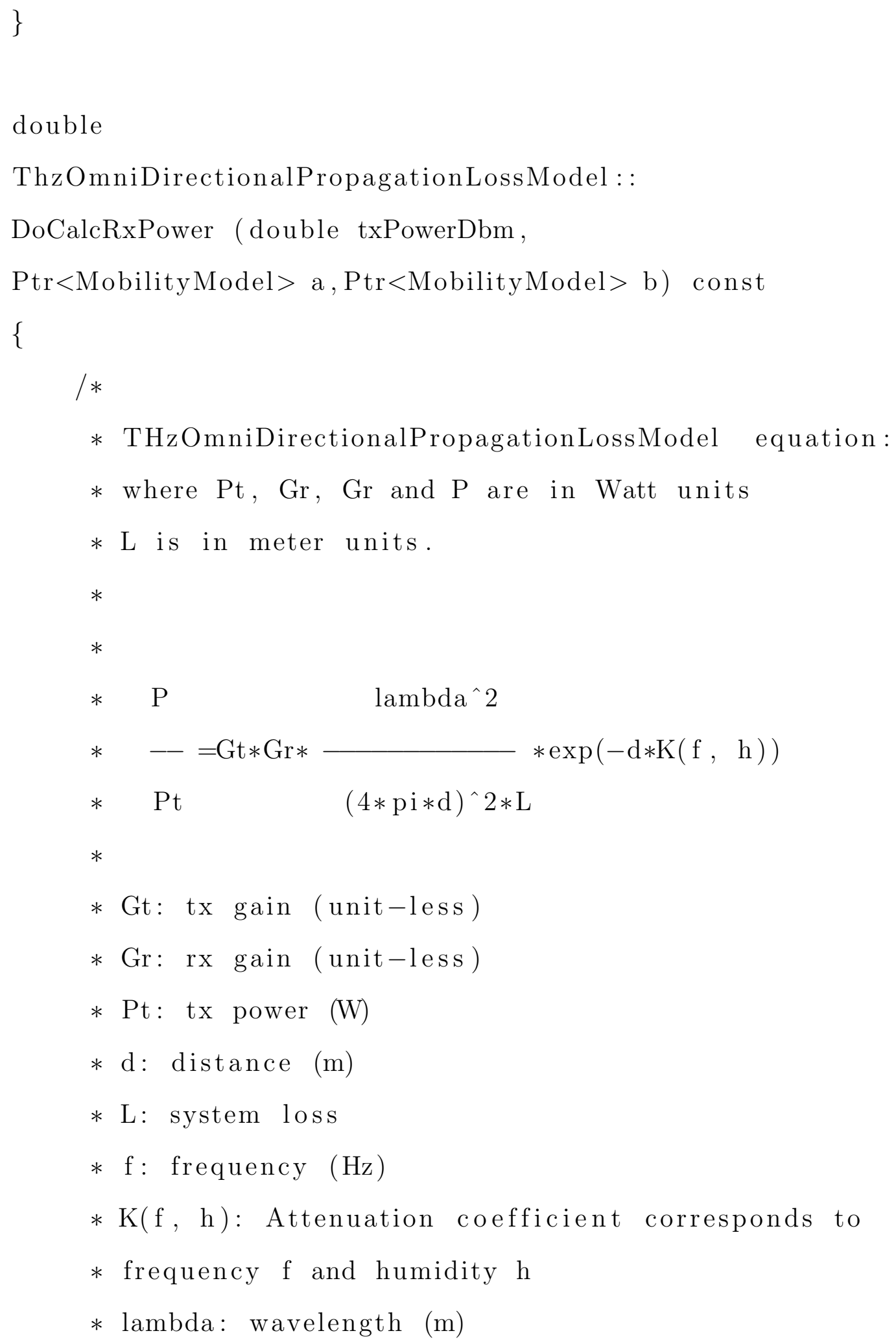




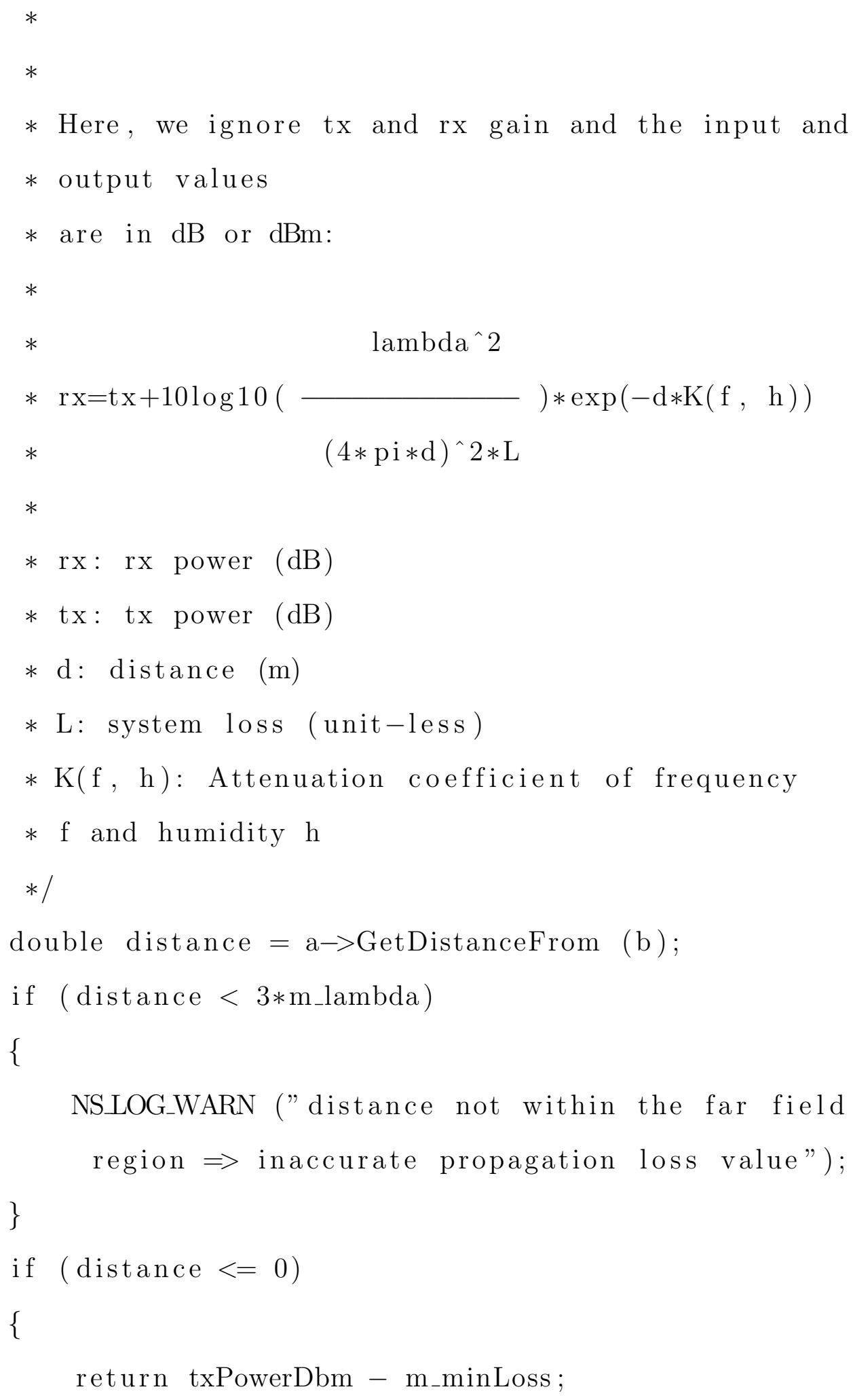




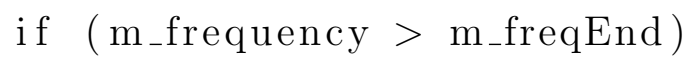




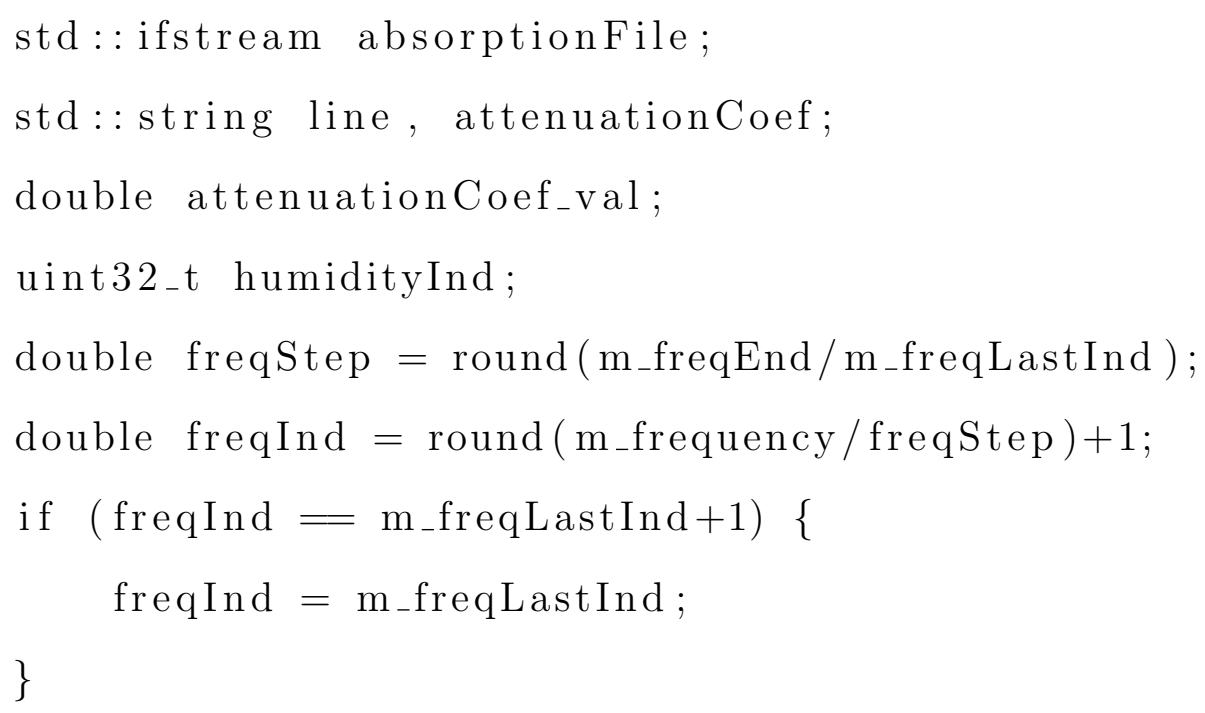




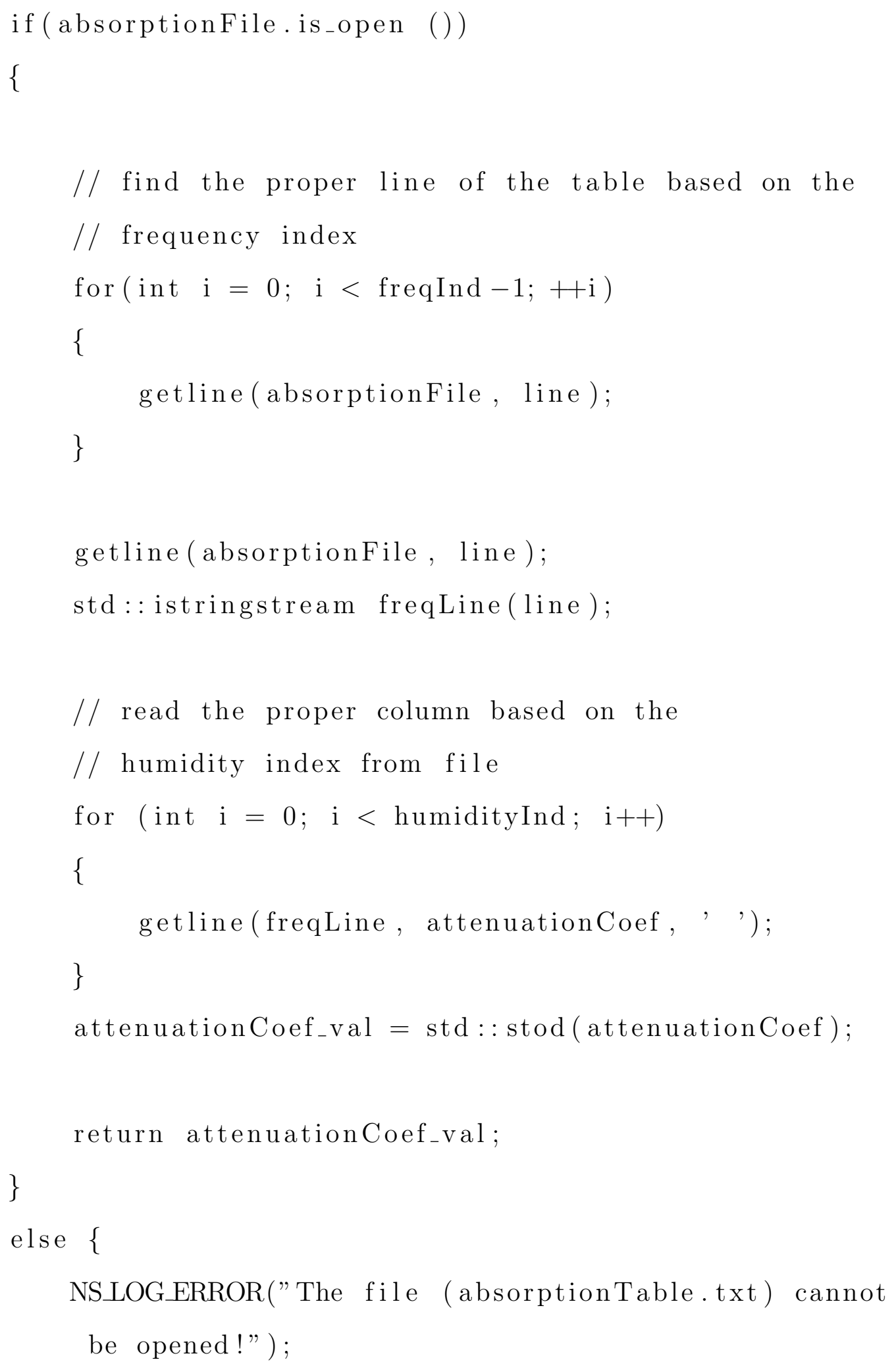




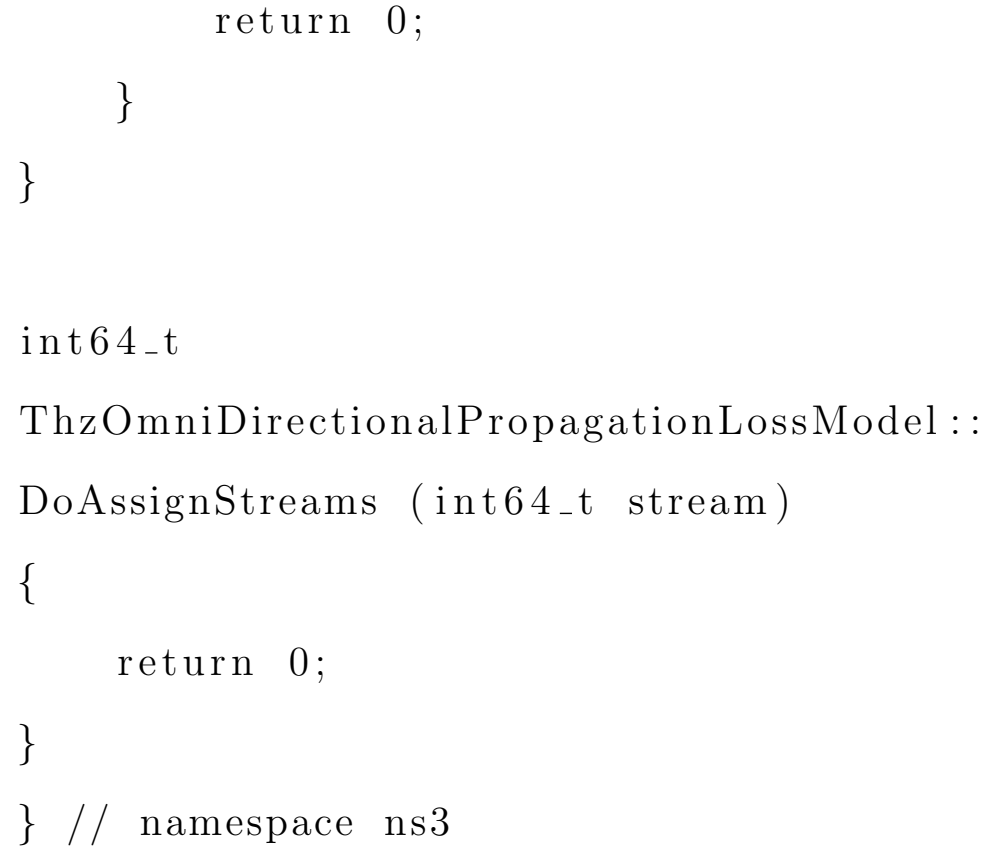




\section{Appendix G}

NS3:THZ PROPAGATION LOSS MODEL TEST

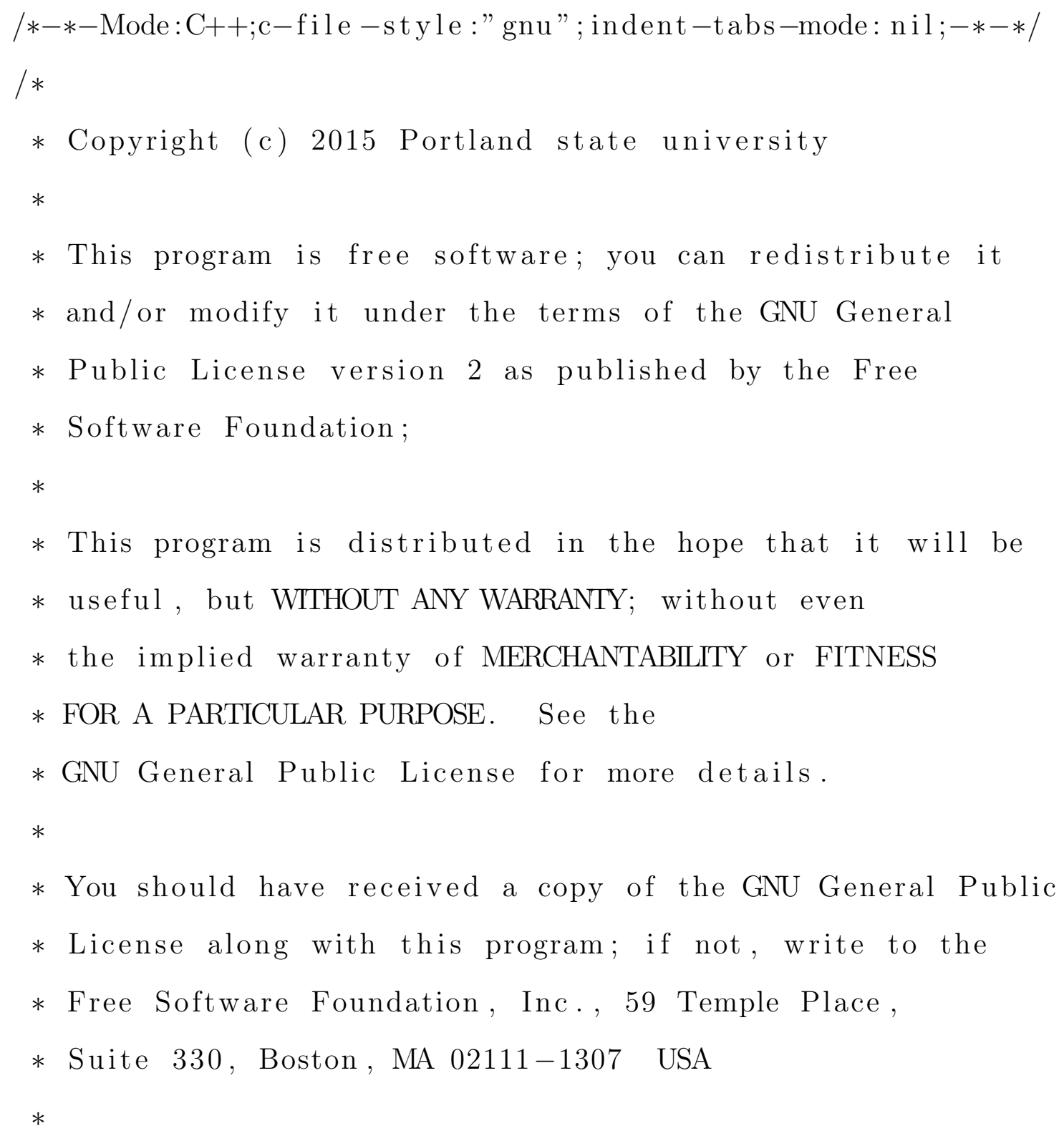


* Author: Farnoosh Moshir <moshir2@cs.pdx.edu>

* $\quad$ Suresh Singh <singh@cs.pdx.edu>

$* /$

\#include "ns3/log.h"

\#include "ns3/abort.h"

\#include "ns3/test.h"

\#include "ns3/thz-propagation-loss-model.h"

\#include "ns3/config.h"

\#include "ns3/double.h"

\#include "ns3/uinteger.h"

\#include "ns3/constant-position-mobility-model.h"

\#include "ns3/simulator.h"

using namespace ns3;

NS_LOG_COMPONENT_DEFINE ("ThzPropagationLoss ModelsTest") ;

// Added for directional THz channels

class ThzDirectionalPropagationLossModelTestCase:

public TestCase

\{

public:

ThzDirectionalPropagationLoss ModelTestCase (); virtual ThzDirectionalPropagationLossModelTestCase (); 
private:

virtual void DoRun (void);

typedef struct \{

Vector m_position;

double m_pt; // dBm

double m_pr; // W

double m_tolerance;

\} TestVector ;

TestVectors $<$ TestVector $>$ m_testVectors ;

\}

ThzDirectionalPropagationLossModelTest Case : :

ThzDirectionalPropagationLoss ModelTestCase ()

: TestCase ("Check to see that the ns-3 THz

directional propagation loss model provides correct

received power"), m_testVectors ()

\{

\}

ThzDirectionalPropagationLoss ModelTest Case : :

$\sim$ ThzDirectionalPropagationLossModelTestCase () 
void

ThzDirectionalPropagationLoss ModelTest Case : :

DoRun ( void)

\{

// Set few defaults.

Config : : SetDefault ("ns3 : :

ThzDirectionalPropagationLossModel : : Frequency" ,

DoubleValue (237.5 e9));

Config : : SetDefault ("ns3 : :

ThzDirectionalPropagationLoss Model : : Humidity", UintegerValue (40));

Config : : SetDefault ("ns3 : :

ThzDirectionalPropagationLoss Model : :

SystemLoss", DoubleValue (1.0));

double txPower $W=0.05035702$;

double txPowerdBm $=10 *$ std : $: \log 10($ txPowerW $)+30$;

// Test the value of the propagation loss model for

// few distances and compare the result with the values

// calculated manually

TestVector testVector; 
testVector.m_position $=$ Vector $(10,0,0)$;

testVector.$m_{-}$pt $=$txPowerdBm;

testVector.m_pr $=0.0504$;

testVector. m_tolerance $=5 \mathrm{e}-5$;

m_testVectors. Add (testVector);

testVector. $\mathrm{m}_{-}$position $=$Vector $(100,0,0)$;

testVector.m_pt $=$ txPowerdBm;

testVector.m_pr $=0.0503$;

testVector.m_tolerance $=5 \mathrm{e}-5$;

$\mathrm{m}_{-}$testVectors. Add (testVector);

testVector.m_position $=$ Vector $(1000,0,0)$;

testVector .m_pt $=$ txPowerdBm;

testVector.m_pr $=0.0502$;

testVector. m_tolerance $=5 \mathrm{e}-5$;

m_testVectors. Add (testVector);

// Now, check that the received power values

// are expected

Ptr $<$ MobilityModel $>a=$

CreateObject $<$ ConstantPositionMobilityModel $>$ ();

$\mathrm{a} \rightarrow$ SetPosition (Vector $(0,0,0))$;

$\operatorname{Ptr}<$ MobilityModel $>\mathrm{b}=$

CreateObject $<$ ConstantPositionMobilityModel $>$ (); 


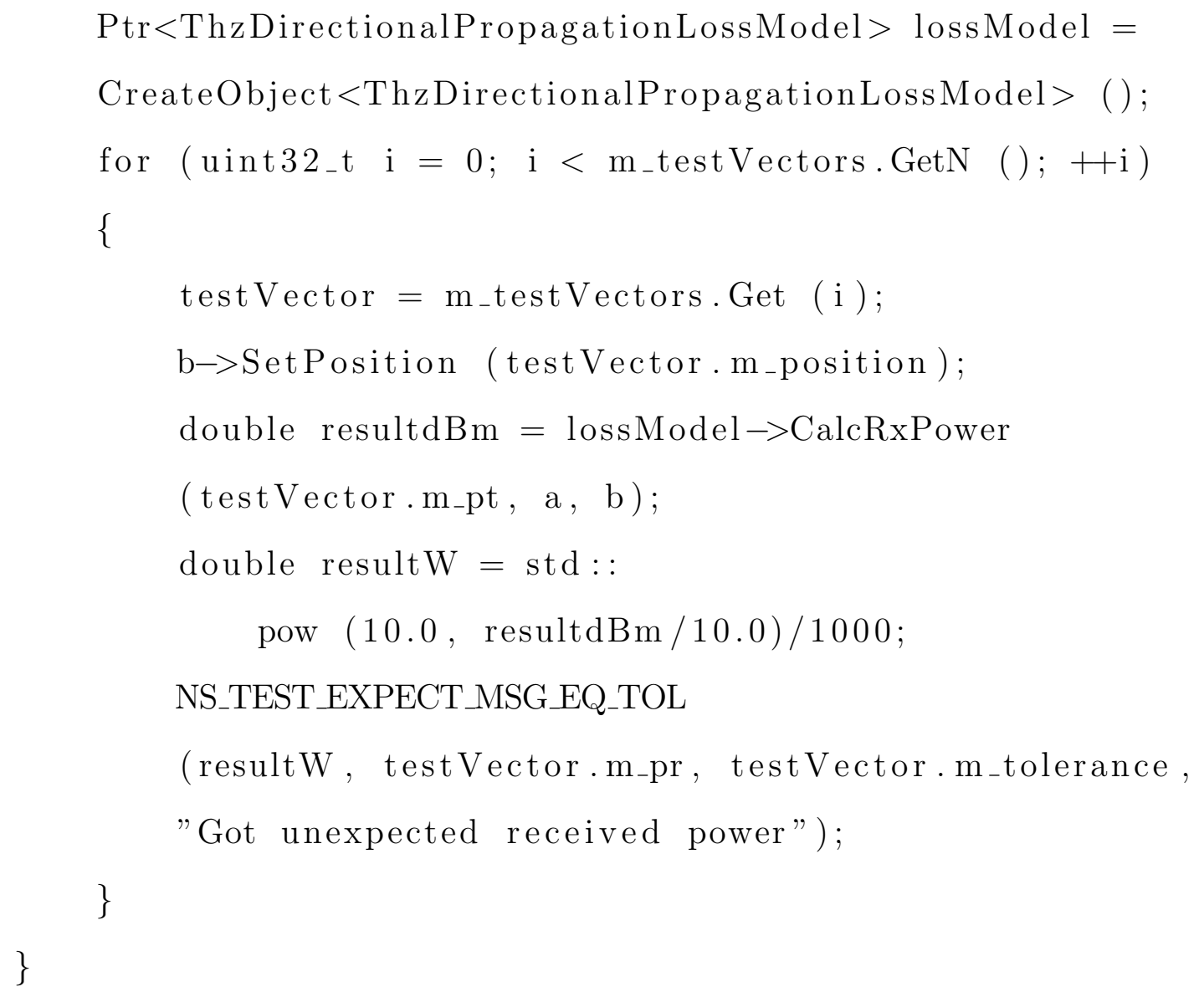


virtual void DoRun (void);

typedef struct \{

Vector m_position;

double m_pt; // dBm

double m_pr; // W

double m_tolerance;

\} TestVector ;

TestVectors $<$ TestVector $>$ m_testVectors ;

\}

ThzOmniDirectionalPropagationLossModelTestCase : :

ThzOmniDirectionalPropagationLoss ModelTestCase ()

: TestCase (" Check to see that the ns-3 THz omni

directional propagation loss model provides correct

received power"),

$\mathrm{m}_{-}$testVectors ()

\{

\}

ThzOmniDirectionalPropagationLossModelTestCase : :

$\sim$ ThzOmniDirectionalPropagationLossModelTest Case () 
void

ThzOmniDirectionalPropagationLoss ModelTestCase : :

DoRun ( void)

\{

// Set few defaults .

Config : : SetDefault ("ns3: :

ThzOmniDirectionalPropagationLoss Model : :

Frequency", DoubleValue (237.5e9));

Config:: SetDefault ("ns3 : :

ThzOmniDirectionalPropagationLossModel : :

Humidity", UintegerValue (40));

Config : : SetDefault ("ns3 : :

ThzOmniDirectionalPropagationLossModel : :

SystemLoss", DoubleValue (1.0));

double txPowerW $=0.05035702$;

double txPowerdBm $=10 *$ std : $: \log 10($ txPowerW $)+30$;

// Test the value of the propagation loss model for

// few distances and compare the result with the values

// calculated manually

TestVector testVector; 
testVector.m_position $=\operatorname{Vector}(5,0,0)$;

testVector.m_pt $=$ txPowerdBm;

testVector.m_pr $=2.0324 \mathrm{e}-11$;

testVector. $m_{-}$tolerance $=5 \mathrm{e}-16$;

m_testVectors. Add (testVector);

testVector.m_position $=$ Vector $(10,0,0)$;

testVector $\cdot \mathrm{m}_{-} \mathrm{pt}=\mathrm{txPowerdBm;}$

testVector.m_pr $=5.0809 \mathrm{e}-12$;

testVector.m_tolerance $=5 \mathrm{e}-17$;

m_testVectors. Add (testVector);

testVector.m_position $=\operatorname{Vector}(100,0,0)$;

testVector.m_pt $=$ txPowerdBm;

testVector.m_pr $=5.0796 \mathrm{e}-14$;

testVector. m_tolerance $=5 \mathrm{e}-19$;

m_testVectors. Add (testVector);

// Now, check that the received power values

// are expected

Ptr $<$ MobilityModel $>\mathrm{a}=$

CreateObject $<$ ConstantPositionMobilityModel $>$ ();

$\mathrm{a} \rightarrow$ SetPosition (Vector $(0,0,0))$;

$\operatorname{Ptr}<$ MobilityModel $>\mathrm{b}=$

CreateObject $<$ ConstantPositionMobilityModel $>$ (); 
Ptr $<$ ThzOmniDirectionalPropagationLossModel $>$ lossModel = CreateObject $<$ ThzOmniDirectionalPropagationLossModel $>$ ( ) ; for $\left(\right.$ uint $32_{-} \mathrm{t} \quad \mathrm{i}=0 ; \mathrm{i}<\mathrm{m}_{-}$testVectors.GetN ()$\left.;++\mathrm{i}\right)$ \{ testVector $=$ m_testVectors. Get (i );

$\mathrm{b} \rightarrow$ SetPosition (testVector.m_position);

double resultdBm $=$ loss Model $\rightarrow$ CalcRxPower (testVector.m_pt, a, b) ; double result $\mathrm{W}=\operatorname{std}:$ : pow $(10.0$, resultdBm/10.0)/1000; NS_TEST_EXPECT_MSG_EQ_TOL（resultW , testVector.m_pr, testVector.m_tolerance, "Got unexpected received power");

\}

\}

class ThzPropagationLossModelsTestSuite : public TestSuite \{ public:

ThzPropagationLoss Models TestSuite ( ); \}

ThzPropagationLoss ModelsTestSuite : : ThzPropagationLossModelsTestSuite ()

: TestSuite ("thz-propagation-loss-model", UNIT) 
AddTestCase (new

ThzDirectionalPropagationLossModelTest Case ,

TestCase : : QUICK );

AddTestCase (new

ThzOmniDirectionalPropagationLossModelTestCase , TestCase : : QUICK) ;

\}

static ThzPropagationLossModelsTestSuite thzPropagationLoss ModelsTestSuite; 
Appendix $\mathrm{H}$

NS3:THZ VS. FRIIS PROPAGATION LOSS MODEL EXAMPLE

/*-*-Mode:C++; c-file-style:" gnu"; indent-tabs-mode: nil; -*-*/

/*

* Copyright (c) 2015 Portland state university

*

* This program is free software; you can redistribute it

* and/or modify it under the terms of the GNU General

* Public License version 2 as published by the Free

* Software Foundation;

$*$

* This program is distributed in the hope that it will be

* useful, but WITHOUT ANY WARRANTY; without even the

* implied warranty of MERCHANTABILITY or FITNESS FOR

* A PARTICUlar PURPOSE. See the GNU General Public

* License for more details.

$*$

* You should have received a copy of the GNU General Public

* License along with this program; if not, write to the Free

* Software Foundation, Inc., 59 Temple Place, Suite 330,

* Boston, MA 02111-1307 USA 
* Author: Farnoosh Moshir <moshir2@cs.pdx.edu>

* $\quad$ Suresh Singh < singh@cs.pdx.edu>

$* /$

\#include "ns3/log.h"

\#include "ns3/test.h"

\#include "ns3/double.h"

\#include "ns3/thz-propagation-loss-model.h"

\#include "ns3/constant-position-mobility-model.h"

\#include "ns3/wifi-mode.h"

\#include "ns3/gnuplot.h"

using namespace ns3;

NSLOG_COMPONENT_DEFINE ("ThzVsFriisLoss");

Gnuplot2dDataset

DoRun $($ Ptr $<$ PropagationLossModel $>$ propagationModel, std : string dataTitle)

\{

Gnuplot2dDataset dataset;

dataset. SetTitle (dataTitle);

dataset. SetStyle (Gnuplot2dDataset : :LINES_POINTS);

double txPowerDbm $=+20$;

double rxPowerDbm; 


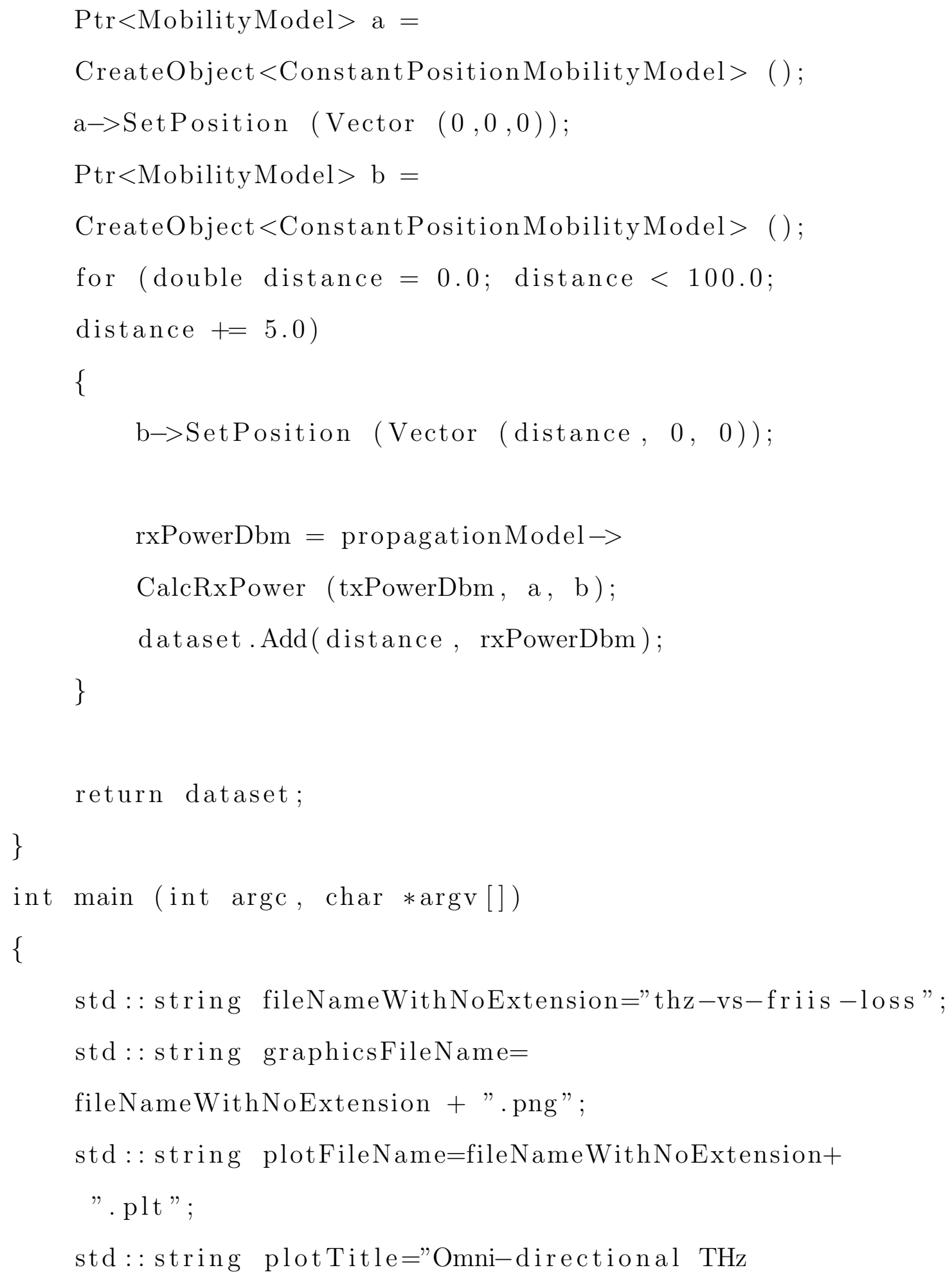


propagation loss vs Friis propagation loss";

Gnuplot plot (graphicsFileName);

plot.SetTitle (plotTitle);

plot.SetLegend ("Distance (m)",

"Recieved Power (dBm)");

Ptr $<$ ThzOmniDirectionalPropagationLossModel $>$ thzPropagationModel $=$

CreateObject $<$ ThzOmniDirectionalPropagationLossModel $>($ );

thzPropagationModel $\rightarrow$ SetFrequency (237.5 e9);

Gnuplot2dDataset dataset $1=$

DoRun(thzPropagationModel, "THz propagation, frequency $=237.5 \mathrm{GHz} ")$;

plot. AddDataset (dataset1);

Ptr $<$ FrisPropagationLossModel $>$ friisPropagationModel $=$ CreateObject $<$ FriisPropagationLossModel $>$ ();

Gnuplot2dDataset dataset $2=$

DoRun(friisPropagationModel, "Friis propagation, default values");

plot.AddDataset (dataset2); 
std : : ofstream plotFile (plotFileName.c_str ());

plot.GenerateOutput (plotFile);

plotFile.close ();

return 0 ; 